\title{
BNL 34518
}

Sixteenth Edition

Informal Report

BROOKHAVEN NATIONAL LABORATORY

Upton, Long Island, New York 11973-5000

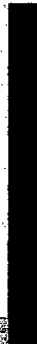

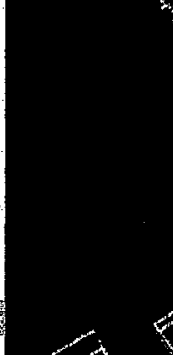

is

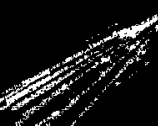

to

BRAHMS
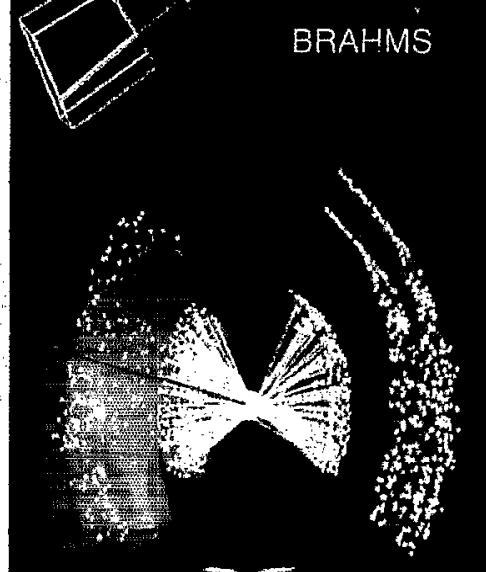

PH ENIX
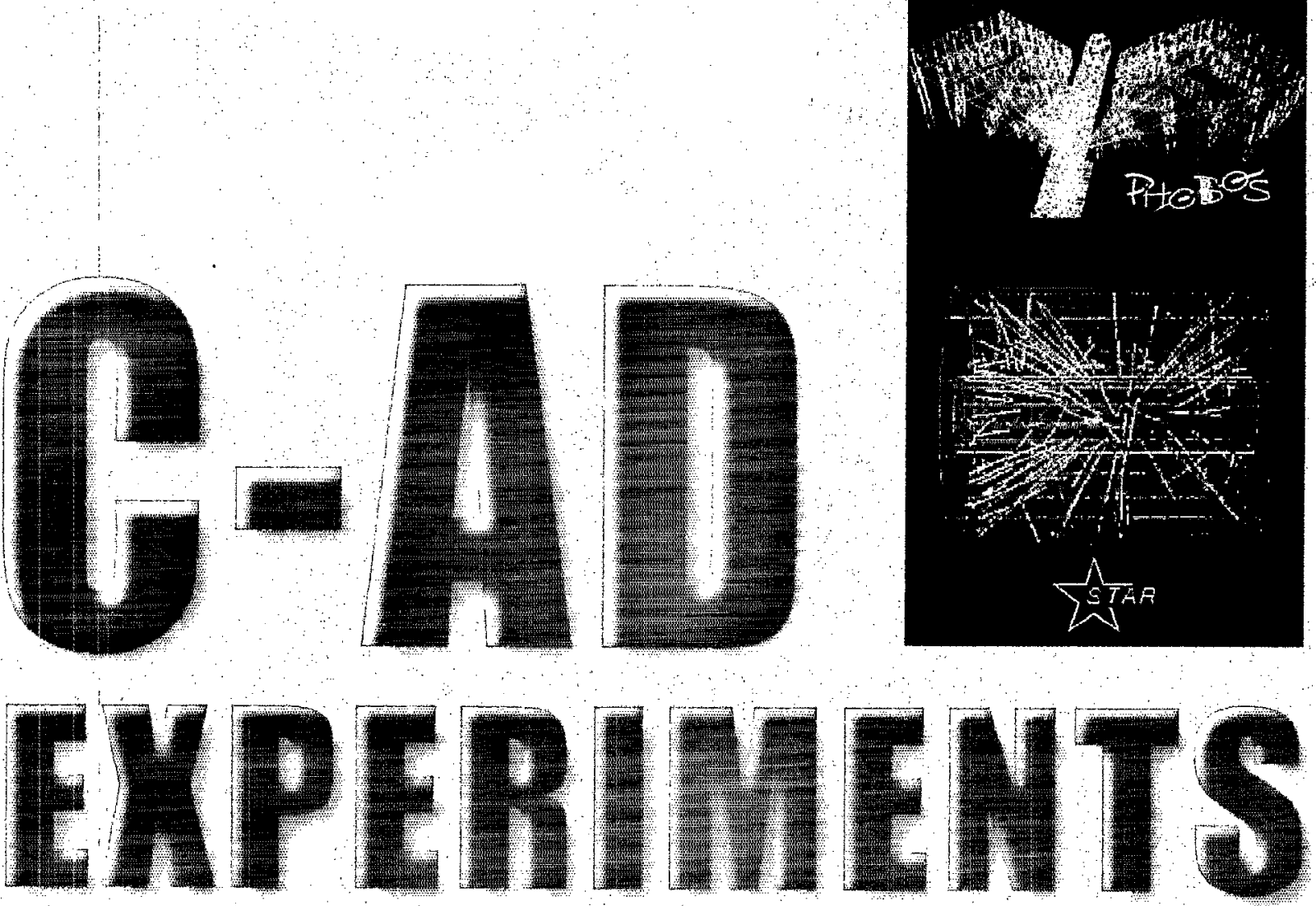


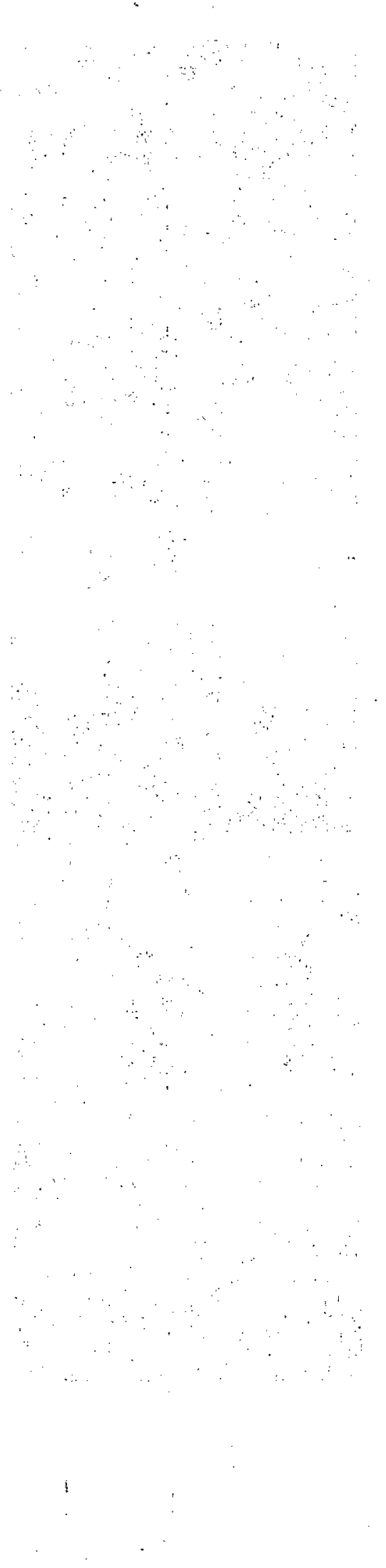


BNL 34518

Sixteenth Edition

Informal Report

C-AD Experiments - $1999-2002$

\title{
P. Lo Presti
}

March 2001

\author{
Collider-Accelerator Department \\ Experimental Support and Facilities Division \\ Brookhaven National Laboratory \\ Brookhaven Science Associates \\ Upton, New York 11973-5000 \\ United States of America \\ Under Contract No. DE-AC02-98CHi0886 with the \\ United States Department of Energy
}

This publication can be located at the following web site: http://server.c-ad.bnl.gov/esfd/eps.htm

Our cover was designed by Peggy Harvey of the C-AD Main Control Room.

"Thank you Peggy for an elegant design." 


\section{DISCLAIMER}

This report was prepared as an account of work sponsored by an agency of the United States Government. Neither the United States Government nor any agency thereof, nor any of their employees, nor any of their contractors, subcontractors, or their employees, makes any warranty, express or implied, or assumes any legal liability or responsibility for the accuracy, completeness or usefulness of any information, apparatus, product, or process disclosed, or represents that its use would not infringe privately owned rights. Reference herein to any specific commercial product, process or service by trade name, trademark, manufacturer, or otherwise, does not necessarily constitute or imply its endorsement, recommendation, or favoring by the United States Government or any agency, contractor, or subcontractor thereof. The views and opinions of authors expressed herein do not necessarily state or reflect those of the United States Government or any agency, contractor or subcontractor thereof. 


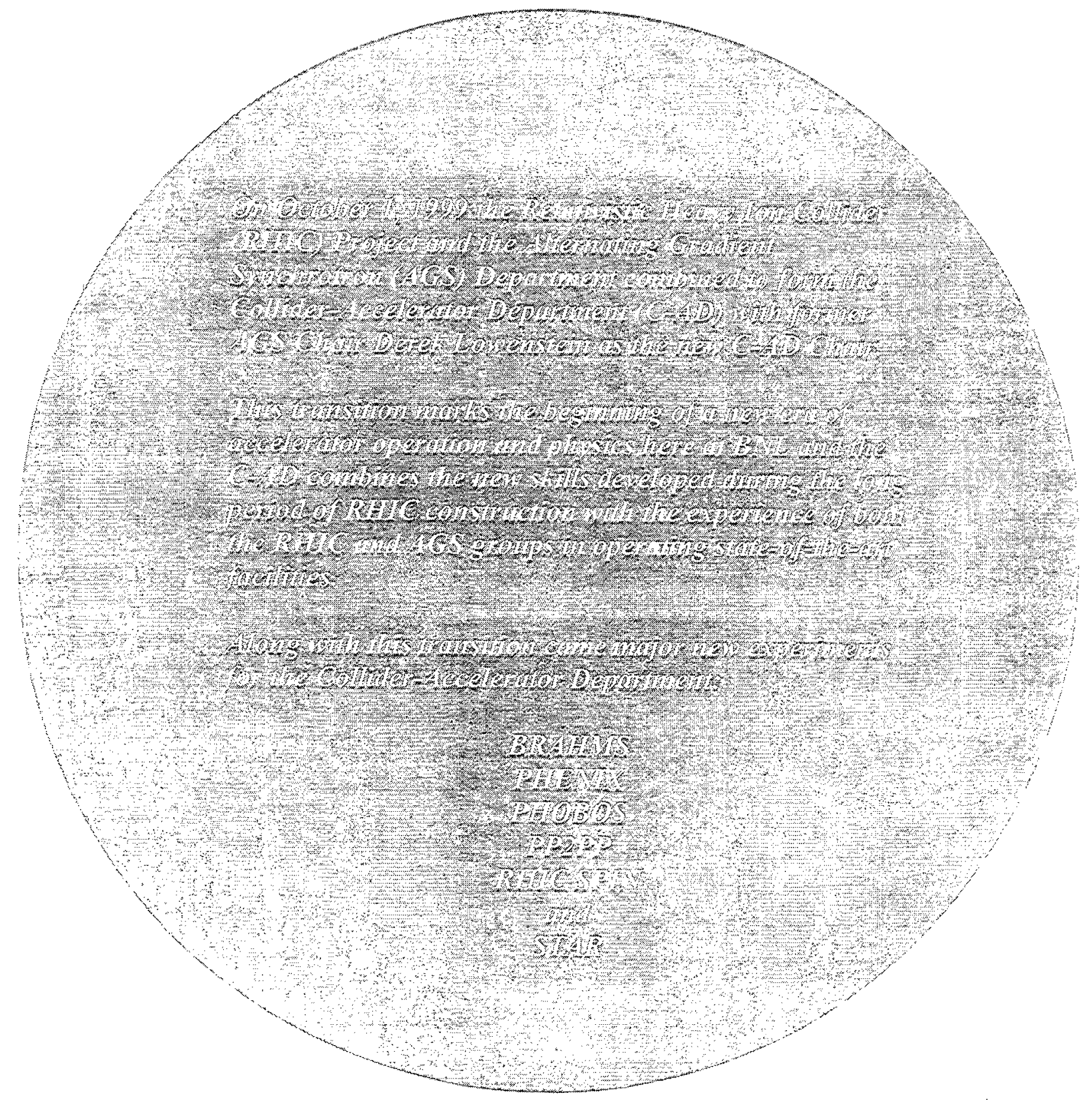





\section{$\underline{\text { Table of Contents }}$}

FY 1999 AGS Schedule as Run

Page:

FY 2000 C-A Schedule as Run

FY 2001 C-A Schedule as Run

FY 2002 C-A Schedule Planned

C-A Complex FY2000-01

C-A Complex FY2002

AGS Experimental Area FY 1998-1999 Physics Program

VII

AGS Experimental Area FY 2000 Physics Program

VIII

AGS Experimental Area FY 2001 Physics Program

IX

AGS Experimental Area FY 2002 Physics Program Proposed

Listing of Experiments

Two-Page Summaries of Experiment

Listing of publications of C-AD experiments

Listing of experimenters in alphabetical order

This is the $16^{\text {th }}$ edition. Please forward suggestions and changes for future editions to:

Division Head, ES\&F Division

Brookhaven National Laboratory

Building 911B

Upton, New York 11973-5000 U.S.A. 

AGS as run schedule, FY1999

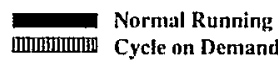

Facility Commissioning

Beam Exp " Experiment Spokesporzon

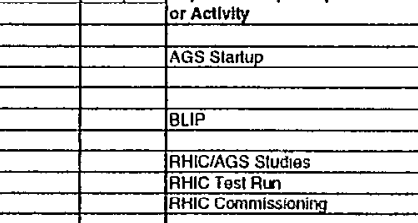

\begin{tabular}{|l|l|l}
\hline & & \\
\hline Ai & $\mathbf{8 5 2}$ & Casonicheryyseth \\
\hline
\end{tabular}

\begin{tabular}{|l|l|l}
\hline A2 & $\mathbf{8 6 5}$ & Zolier \\
\hline
\end{tabular}

\begin{tabular}{|c|c|l|}
\hline & & \\
\hline A3 & 941 & HuangiMalu \\
\hline A3 & 947 & Vazquaz \\
\hline
\end{tabular}

\begin{tabular}{rr|r|r}
\hline B1 & 925 & Makdisirokosawa \\
\hline
\end{tabular}

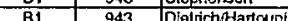

\begin{tabular}{|l|l|l}
\hline B2 & & ToslBeam \\
\hline
\end{tabular}

\begin{tabular}{|c|c|c|}
\hline 61 & 850 & Camouriteppoiman \\
\hline
\end{tabular}

\begin{tabular}{|c|c|c|}
\hline C4 & 787 & 80ymancilitenbernismith \\
\hline
\end{tabular}

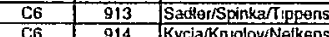

\begin{tabular}{|c|c|c|}
\hline $\mathrm{C6}$ & 914 & Kyclandikens \\
\hline $\mathrm{C} 6$ & 927 & Ky \\
\hline $\mathrm{C} 6$ & PHOBOS & Sleachan \\
\hline
\end{tabular}

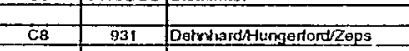

\begin{tabular}{lll}
\hline 06 & 906 & Chrienifukudarifusek \\
\hline
\end{tabular}

\begin{tabular}{|l|l|l}
\hline 06 & 930 & Tamura \\
\hline
\end{tabular}

\begin{tabular}{|c|c|c|}
\hline$U$ & 938 & Eavertitaines Hastingsi Walanabo \\
\hline
\end{tabular}

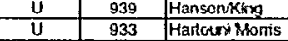

U 945 Cooperimorgan

\begin{tabular}{|c|c|c|c|}
\hline$U$ & $945 A$ & Greon \\
\hline$U$ & $945 B$ & Groes \\
\hline
\end{tabular}

\begin{tabular}{|c|c|l}
\hline $\mathrm{V}_{1}$ & 821 & Huohesmorsainoberts \\
\hline
\end{tabular}

\begin{tabular}{|c|c|c|}
\hline AGS & 950 & IraüFidys \\
\hline
\end{tabular}
By: W. Ment/H. Huang, Dale: 1 Oug

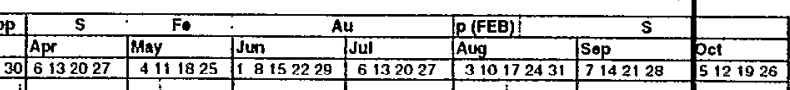

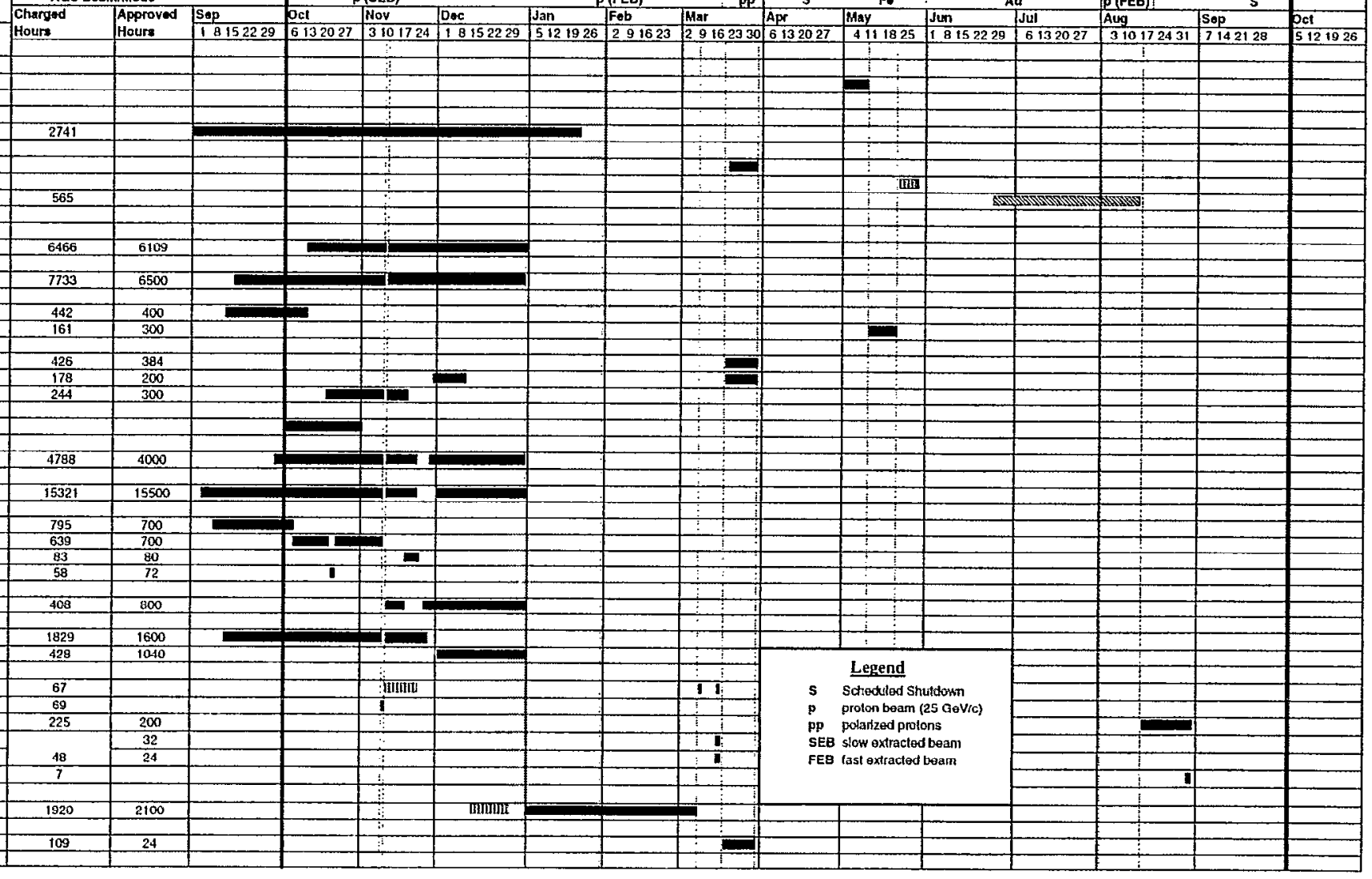




\section{C-A as run schedule, FY2000}

Norntal Running

(a)

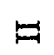

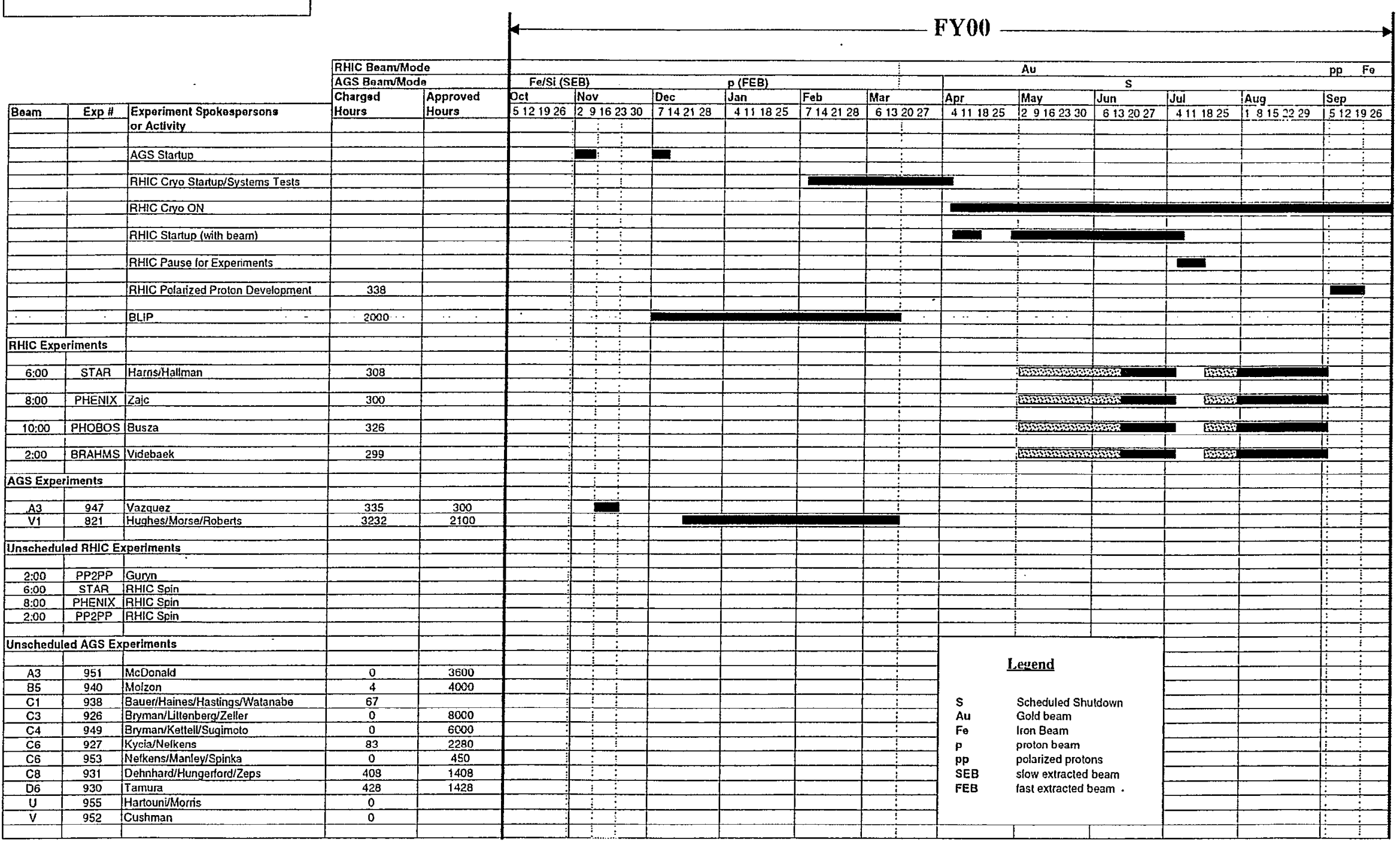
By: Y. Makdisi. Dante: 30 Sip 00
colrected $10 / 120101$

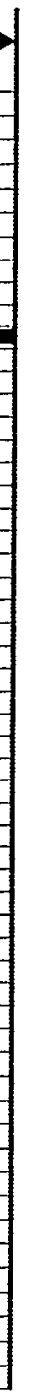




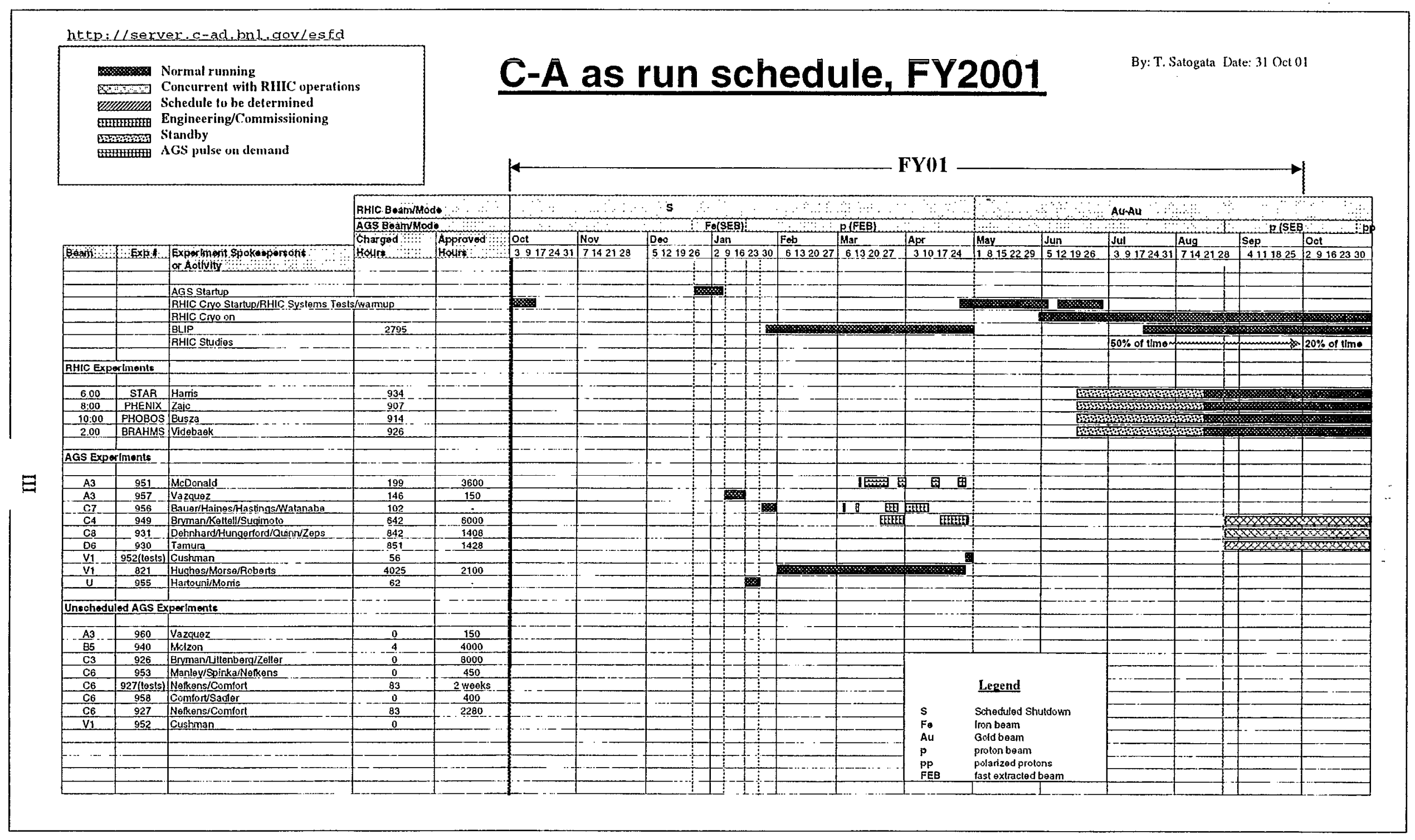


hetp: /lserver.c-ad hnl gov/esfd

Normal running

Concurrent with RHIC operations

TRTITOR Schedule to be deternined

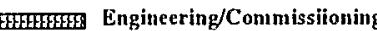

Standby

\section{C-A Run Plan, FY2002}

By: K. Drees/P. Pile Date: 18 Dec 01 hours as of 13 Nov 01

\section{In progress, subject to funding and other issues}

FY02

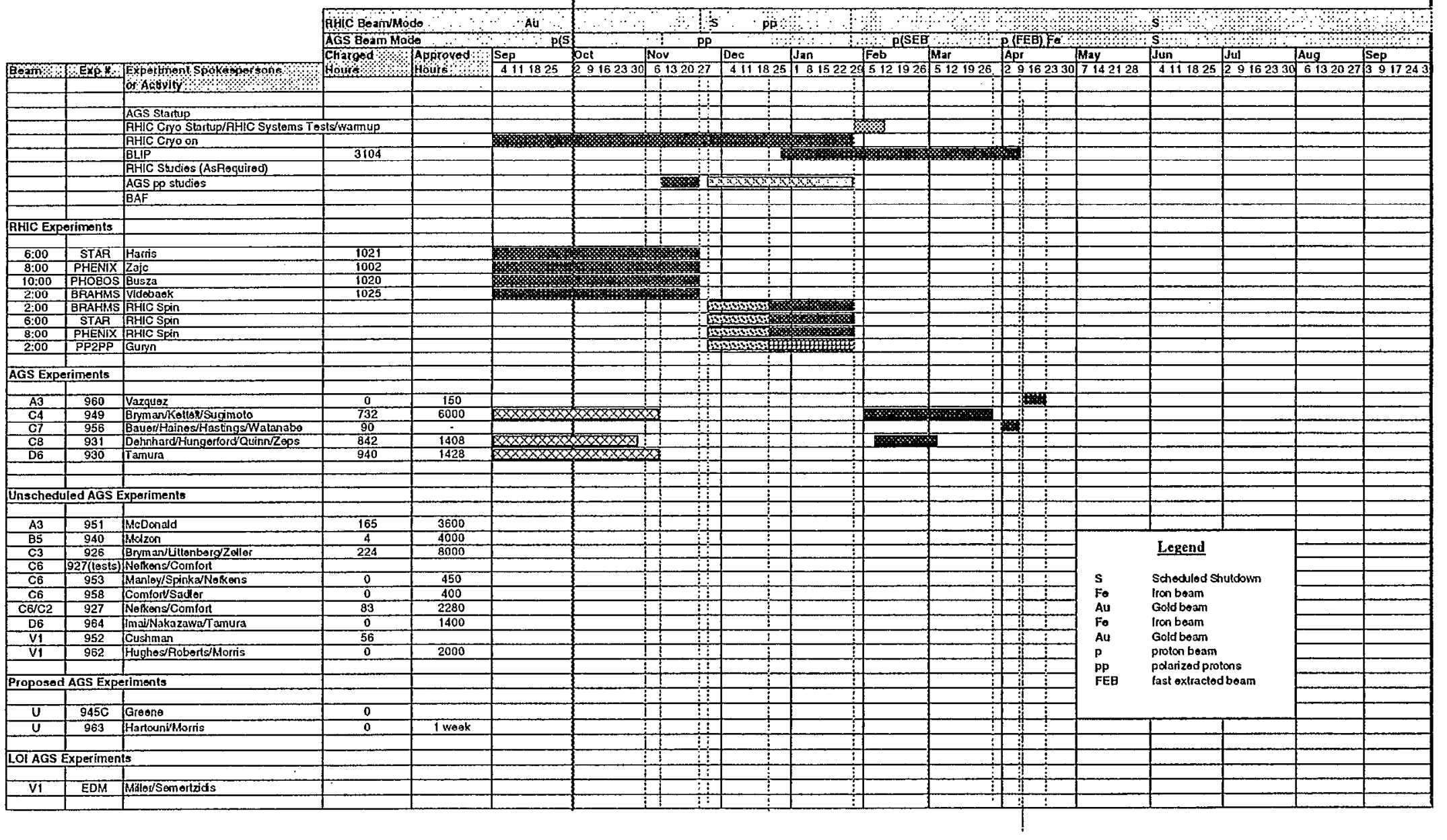




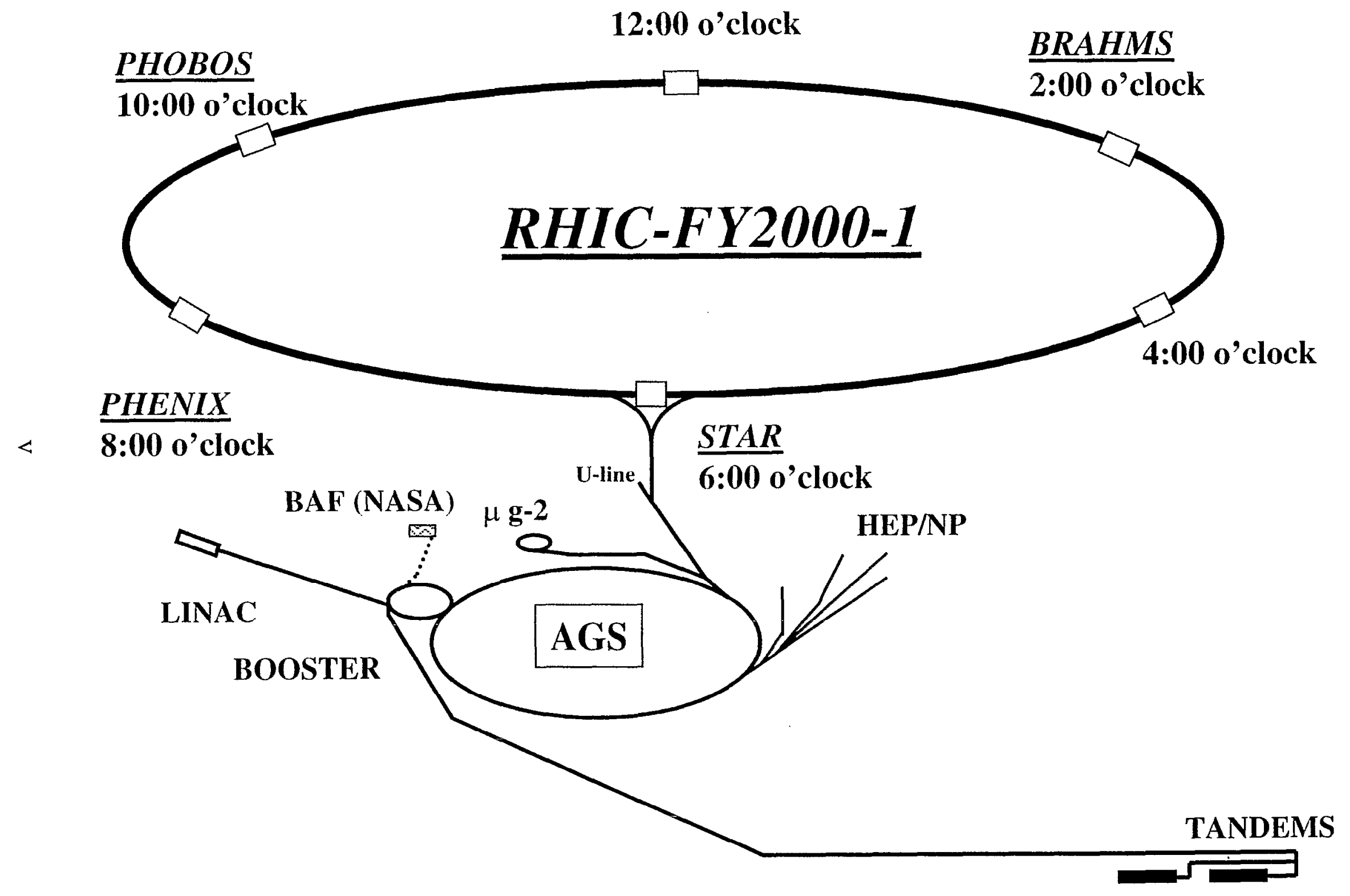




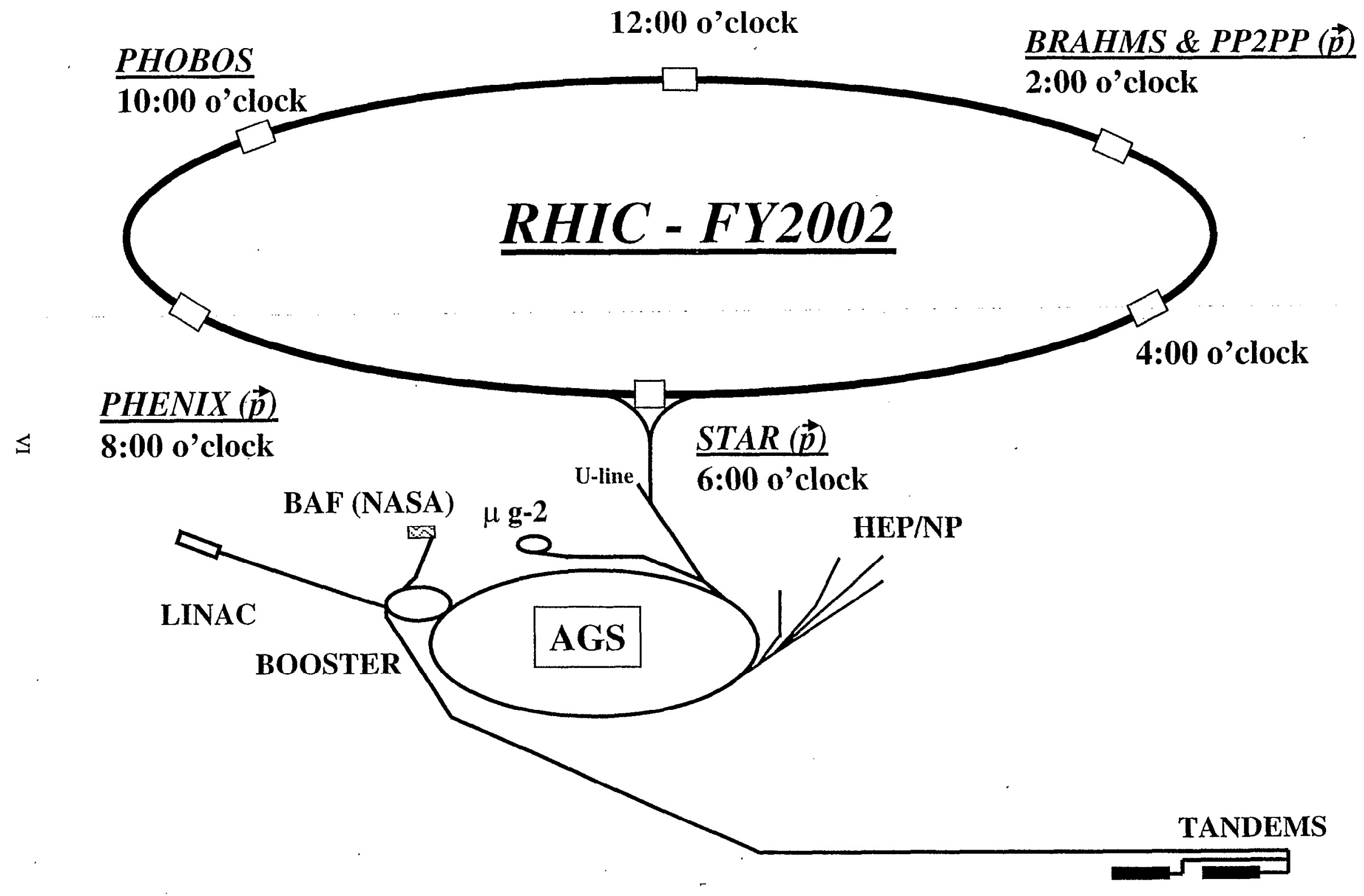




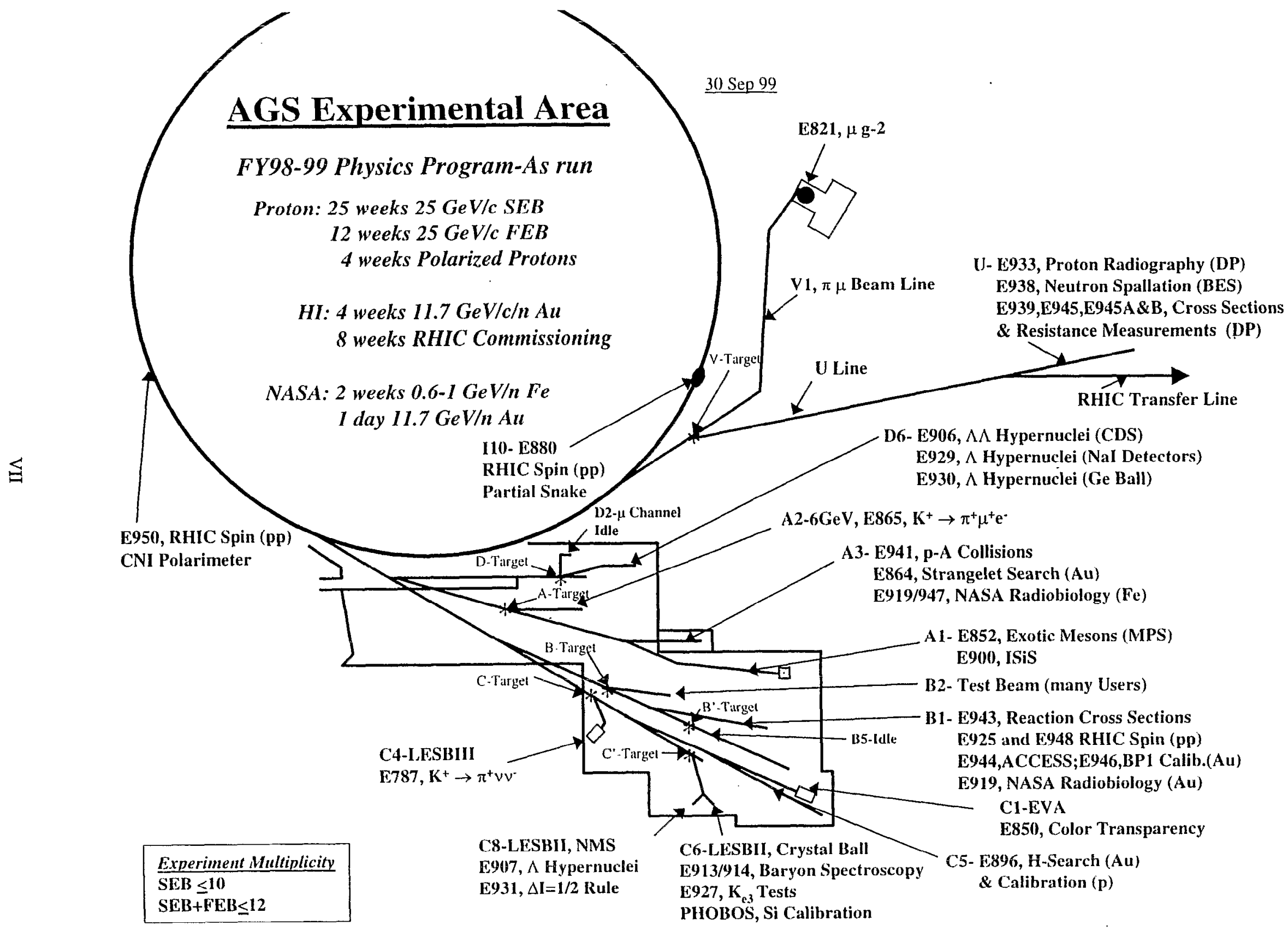




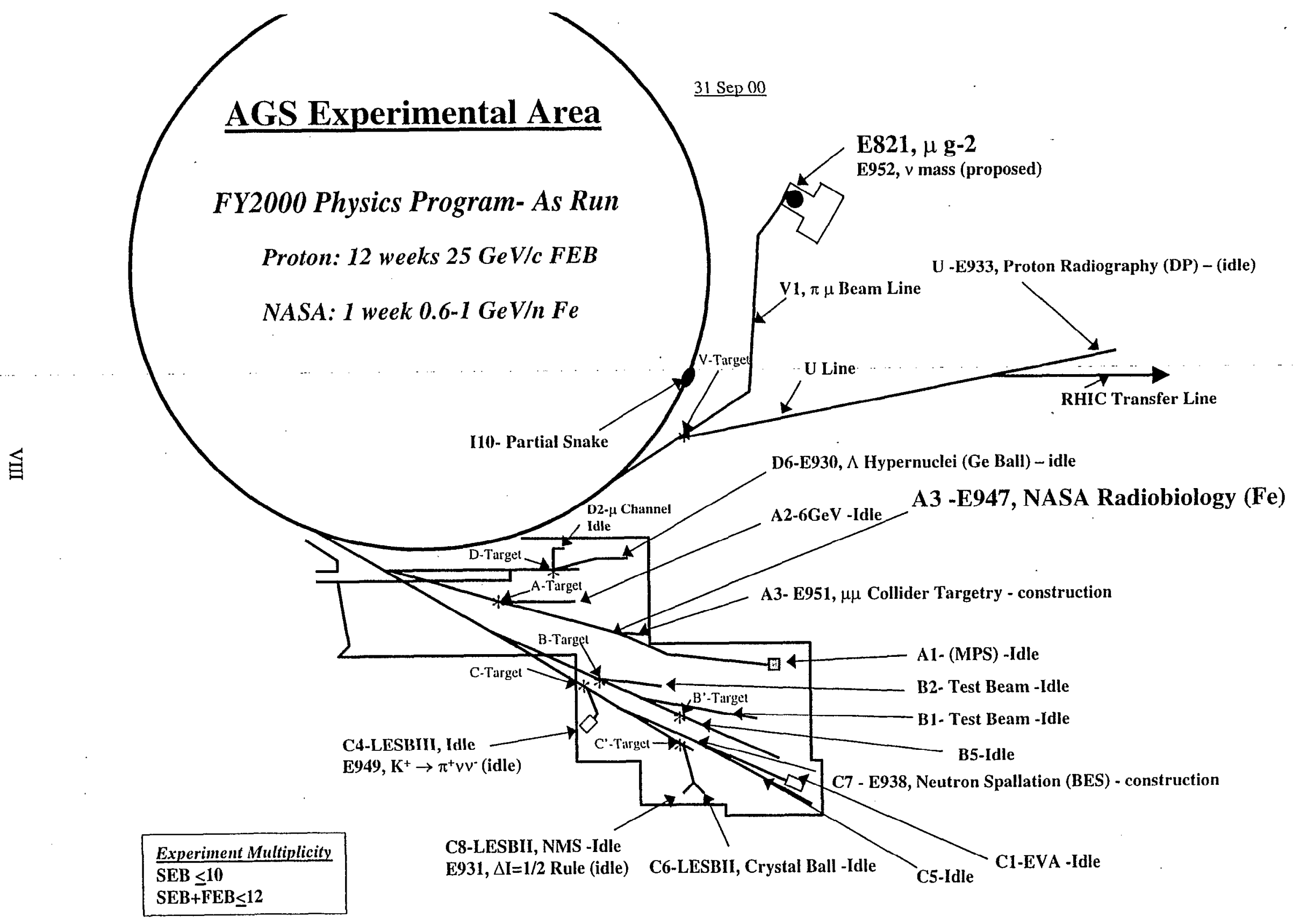




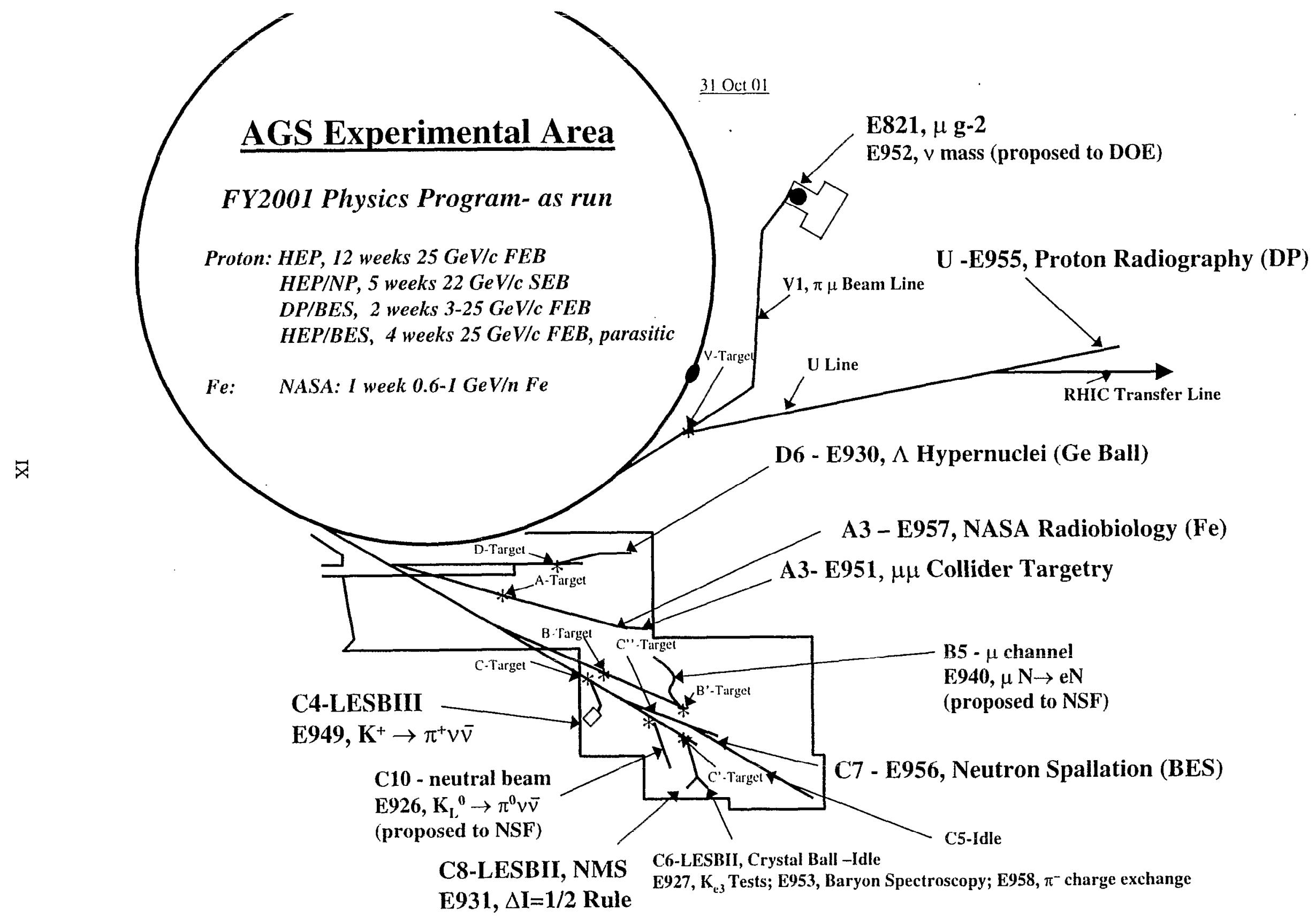




\section{AGS Experimental Area}

\section{FY2002 Physics Program- In progress/planned}

Proton: HEP, 12 weeks 20-22 GeV/c SEB

NP, 4 weeks 20-22 GeV/c SEB

NP, 4 weeks 20-22 GeV/c SEB (parasitic)

HEP/BES/NNSA, 3-25 GeV/c FEB (TBD)

Fe: NASA: 1 week 0.6-1 GeV/n Fe

10 Dec 01

V1-Idle: E952, v mass (proposed to DOE) E962, $\mu$ g-2 (proposed to DOE)

U- E963, proton radiography E945C, Radiation effects

$V 1, \pi \mu$ Beam Line

Line

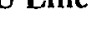

D6 - E930, $\Lambda$ Hypernuclei (Ge Ball)

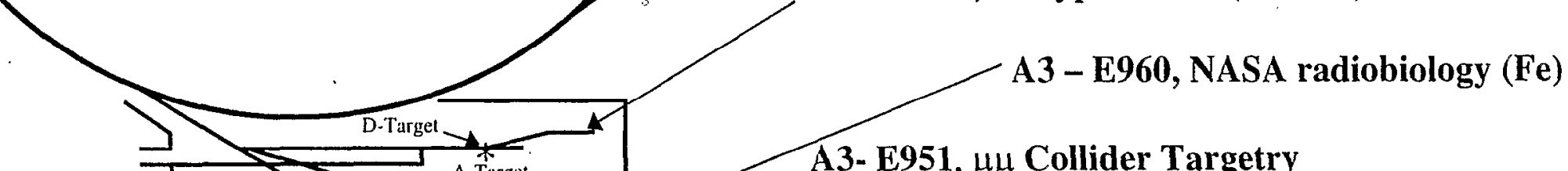

A3- E951, $\mu \mu$ Collider Targetry

C4-LESBIII

E949, $\mathrm{K}^{+} \rightarrow \pi^{+} \nu \bar{v}$

C10 - neutral beam E926, $\mathbf{K}_{\mathrm{L}}{ }^{0} \rightarrow \pi^{0} v \bar{v}$ (proposed to NSF)

C8-LESBII, NMS E931, $\Delta \mathrm{I}=1 / 2$ Rule B5 - $\mu$ channel E940, $\mu \mathrm{N} \rightarrow \mathrm{eN}$ (proposed to NSF) C-Target
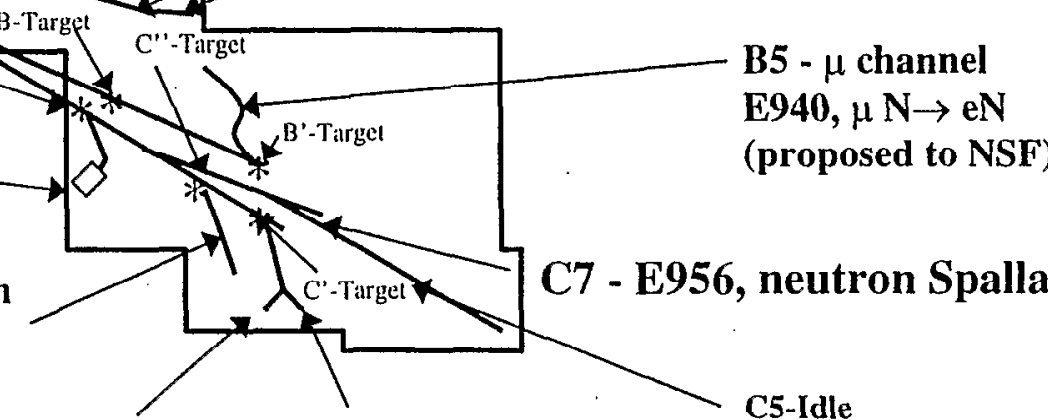

C7 - E956, neutron Spallation (BES) C6-LESBII, Crystal Ball-Idle E927, $K_{e 3}$ tests; E953, baryon spectroscopy;

E958, $\pi^{-}$charge exchange 


\section{LIST OF EXPERIMENTS}

$\operatorname{Exp}$

BRAHMS B̧road Range Hadron Magnetic Spectrometers

BNL/U. Bucharest/Jagellonian U/Johns Hopkins U/NYU/U. Copenhagen/

Texas A\&M/Fysisk Inst/U. Bergen/ U. Kansas/U. Lund/U. Olso

Videbaek, Gaardhoje

PP2PP

BNL/Ecole Polytechnique/U. Genova/Inst. of Nuclear Science, Grenoble/

U. Iowa/Moscow Eng/Phys. Int/U. Padova/IHEP/SUNY Stony Brook/

U. Texas/Tomsk Nuclear Phys.Int/U. Trieste \& Sezione

Guryn

PHENIX

Collaboration (http://www.phenix.bnl.gov/phenix/WWW/html/phenix_inst.html) Zajc

PHOBOS

ANL/BNL/INP, Krakow/Jagiellonian U/MIT/Natl. Central U, Taiwan/

U. Rochester/ U. Ill/U. Md

Busza

SPIN

Collaboration (http://www.agsrhichome.bnl.gov/RHIC/Spin)

Imai, Moss

STAR Solenoid Tracker $\underline{\text { At }} \underline{\mathrm{R}} \mathrm{HIC}$

Collaboration (http://www.star.bnl.gov/STAR/spb/institute.html)

Harris

787

A Study of the Decay $\mathrm{K}^{+} \rightarrow \pi^{+} v \bar{v}$

BNL/Fukui/KEK/Osaka/Princeton/TRIUMF/Alberta

Bryman, Littenberg, and Smith

821

A New Precision Measurement of the Muon g-2 at the Level of 0.35 PPM

Boston/BNL/Budker Institute/Cornell/Fairfield/Heidelberg/Illinois/LBL/

Max Planck Inst/Minnesota/KEK/Tokyo/Yale

Hughes, Morse, and Roberts

The Effects of a Partial Siberian Snake on Polarization at the AGS

IUCF/BNL/ANL/TRIUMF/Fermilab/KEK/THEP-Serpukhov/Riken

Lee and Roser

896

A Proposal to the BNL AGS: To Search for a Short-Lived $\mathrm{H}_{\mathbf{0}}$ Dibaryon, Short-Lived Strange Matter, and to Investigate Hyperon Production in 11.6 A GeV/c AuAu Collision

SSL/BNL/UCLA/Carnegie-Mellon/Catania/CERN/Johns Hopkins/LBL/ Michigan/NASA-Goddard Space Flight Center/Ohio St/Texas-Austin/ Rice/Wayne St/Yale

Crawford and Hallman 
Abilene Christian/ANL/Arizon State U/BNL/UCLA/G. Washington/

Kent St/ PNPI-Gatchina/R. Boskovic Inst/Colo/Karlsruhe/Regina/Valparaiso Nefkens, Kycia, and Kruglov

A Study of the Genetic and Epigenetic Effects Produced by High Energy Heavy Ions

BNL/LBNL/Colorado St/USAF Armstrong Lab/Maryland-Baltimore County/

Maryland-Baltimore/Georgetown U. Medical Center/Loma Linda/LANL/

NASA-Johnson Space Ctr/UC/Columbia/Pacific NWNL/Washington St/

Case Western Reserve/NIRS-Japan/Texas/Alabama Agr. \& Mech.

Vazquez

Search for T Violating Muon Polarization in $\mathrm{K}^{+} \rightarrow \mu^{+} \pi^{0} v_{\mu}$ Decay

BNL/INR-Moscow/Louisiana Tech/Yale Adair, Diwan and $M a$

K0PI0, Measurement of $\mathrm{K}_{\mathrm{L}}^{\mathrm{o}} \rightarrow \boldsymbol{\pi}^{0} v \bar{v}$

BNL/INR-Moscow/Kyoto/New Mexico/Thomas Jefferson National Accelerator Facility/TRIUMF/Yale/U.British Columbia/VPI Bryman, Littenberg and Zeller

BNL/UCLA/JINR/Abilene Christian/ANL/Arizona State/Rudjr

Boškovič Inst/U.Colo/Geo.Washington U/ U.Karlsruhe/ Kent/

SPNP/U.Regina/Valparaiso

Kycia and Nefkens

High-Resolution $\gamma$ Spectroscopy of Hypernuclei Using Large-Acceptance

Germanium Detector

Tokyo/Osaka/KEK/Tohoku/BNL/North Carolina A\&T/Hampton

Tamura 
LANL/LLNL/Bechtal Morris and Hartouni BNL/LLNL/LANL/DOE/Bechtel Nevada/CEA/LNS-CNRS King and Hanson

UC-Irvine/Houston/Inst.Nuclear Research/NYU/Pennsylvania/Purdue Molzon

945A Proposal for a Radiaton-Damage Experiment for the Accelerator Production of Tritium (APT) Project at the AGS

BNL/LANL

$$
\text { Greene and Sommer }
$$

BNL/LANL Greene

NASA Radiobiology

BNL/Case Western/Columbia/Colo/Duke/LBNL/NASA Johnson Space Ctr/Natl/ Space biology Res. Inst/Loma Linda/NYU Med/U. Tex.Med/T. Jeff.U/LANL/ Wash U/Natl. Inst. Radiological Sciencs,Japan/U.di Bologna,Italy

\section{Burns}

BNL /KEK/Osaka U/Triumf/U. Alberta Bryman, Kettell, Sugimoto ANL/BNL/CERN/Fermi/LBNL/ORNL/Jos.Henry Lab, Princeton U/SUNY Stony Brook McDonald 
Exp.

953

Neutral Hyperon Spectroscopy with the Crystal Ball

Kent/Abilene Christian U/ANL/Az U/UCLA/Colo U/GWU/U. Karlsruhe/

U. Md/PNPI/Rudjer Boskovic Inst/U. Uppsala, Sweden/Valparaiso

Manley, Nefkens, Spinka

955

Proton Radiography at the AGS

Bechtal Nevada/BNL/LANL/LLNL

Hartouni, Morris

Spallation Neutron Sutdies at the AGS

ANL/BNL/FZJ/JAERI/LANL/ORNL/PSI

Bauer, Haines, Hastings, Watanabe

957

NASA Ridiobiology

BNL/Case Western/Columbia/Colo/Duke/LBNL/NASA Johnson Space Ctr/

Natl. Space biology Res. Inst/Loma Linda/NYU Med/U. Tex.Med/T. Jeff.U/

LANL/Wash U/Natl. Inst. Radiological Sciencs, Japan/U.di Bologna,Italy

Burns

958

Pion Charge-Excahnge Cross-Sections at Low Energies

Abilene Christian/ANL/Ariz/UCLA/Colo/GWU/Karlsruhe/Kent/U.Md/PNPI/U.

Regina/Rudjer/Valapraiso/Uppsala

Comfort/ Sadler

BNL/Case Western/Columbia/Colo/Duke/LBNL/NASA Johnson Space Ctr/ Natl. Space biology Res. Inst/Loma Linda/NYU Med/U. Tex.Med/T. Jeff.U/

LANL/Wash U/Natl. Inst. Radiological Sciencs,Japan/U.di Bologna,Italy

Burns

Publications

C-AD Experimenters 
Summary

of

Experiments 


$$
\text { . }
$$





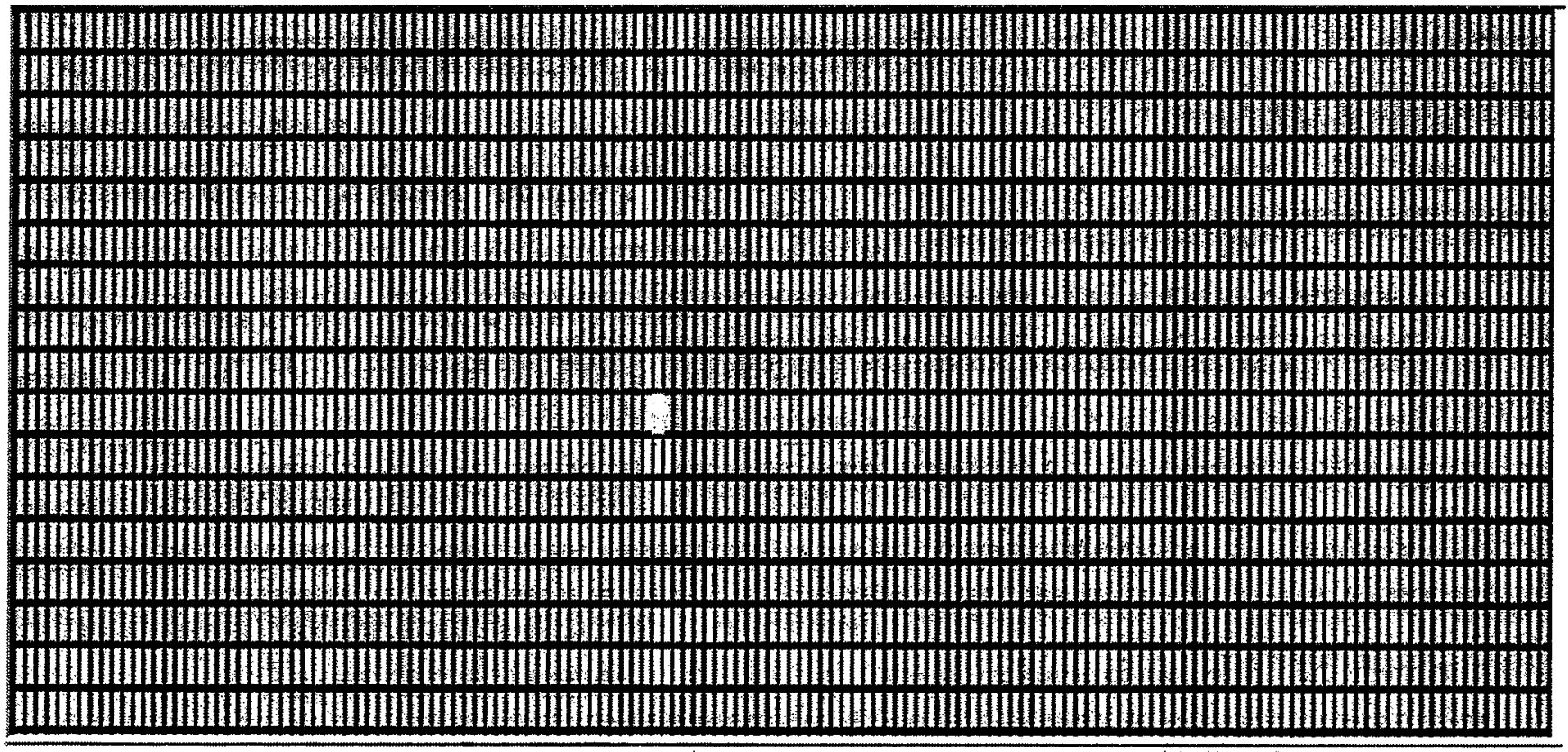

At approximately 23:00 on June 15, 2000 detection was made of the first collisions. 


\section{EXPERIMENT-BRAHMS}

\section{SPOKESMEN: F. VIDEBAEK AND J. J. GAARDHOJE}

- Brookhaven National Laboratory - D. Beavis, C. Chasman, R. Debbe, J. H. Lee, E. McBreen, K. Olchanski, J. W. Olness, R. Scheetz, F. Videbaek

- Fysisk Institutt - K. Fanebust, R. Gruner, D. Roehrich

- Jagellonian University - K. Grotowski, T. Kozik, Z. Majka, Z. Sosin, P. Staszel

- Johns Hopkins University - E. J. Kim, Y. K. Lee

- New York University - Y. Blyakhman, B. Budick

- Niels Bohr Inst. for Astronomy, Physics and Geophysics University - J. G. Bearden, J. P. Bondorf,

H. Boggild, J. J. Gaardhoje, A. G. Hansen, O. Hansen, A. Holm, C. Holm, H. Heiselberg,

B. Svane Nielsen

- Texas A\&M University - C. Cibor, K. Hagel, M. Murray, J. Natowitz, R. Wada

- University Bucharest - C. Besliu, D. Argintanu, R. Zaharia, F. Constantin, D. Felea, A. Jipa, I. S. Zgura

- University Kansas - H. Ito, S. J. Sanders

- University of Lund - B. L $\mathrm{L}^{\mathrm{a}}$ orstad, J. Schmidt-Sorensen

- University Oslo - G. Lovhoiden, A. K. Holme, T. S. Tveter

BRAHMS, the Broad RAnge Hadron Magnetic Spectrometers experiment at RHIC is designed to measure charged hadrons over a wide range of rapidity and transverse momentum to study the reaction mechanisms of the relativistic heavy ion reactions at RHIC and the properties of the highly excited nuclear matter formed in these reaction. 


\section{ELASTIC SCATTERING (BASIC FACTS)}

1. Elastic scattering process: In State $=$ Out State, $p p \rightarrow p p$

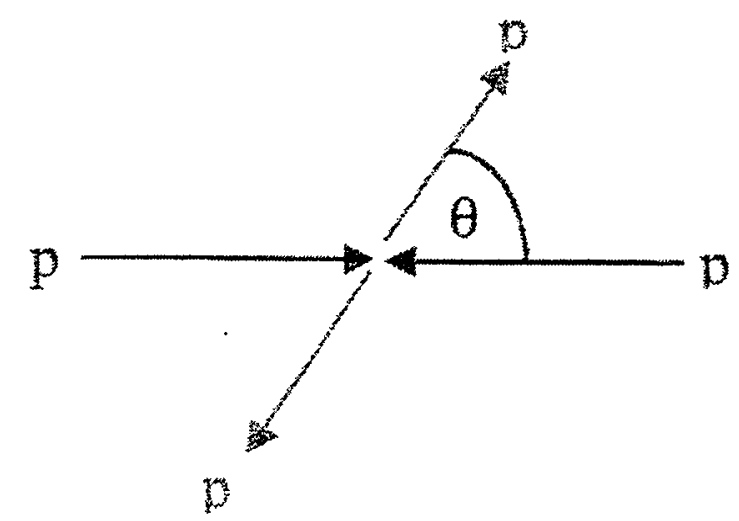

2. Kinematic variables:

a) $t=$ four momentum transferred squared

$$
\begin{aligned}
& t=\left(p_{\text {out }}-p_{\text {in }}\right)^{2}=-2 p^{2}(1-\cos \theta) \\
& t \cong-p^{2} \theta^{2} \text { for small angles }(80 \mu \mathrm{rad}<\theta<13 \mathrm{mrad}) \\
& t \text { - gives the scattering angle in the CMS }
\end{aligned}
$$

b) $s=4\left(p^{2}+m^{2}\right) \cong 4 p^{2}$

$$
\sqrt{s}=\text { Total CMS Energy }
$$


- Brookhaven National Laboratory - R. Chrien. R. Gill, W. Guryn, D. Lynn, A. Rusek, M.Sakitt, S. Tepikian

- Ecole Polytechnique - J. Bourotte, M. Haguenauer

- Institute des Science Nucleaires - M. Buenerd

- Institute for High Energy Physics - A. Ufimtsev

- Moscow Engineering Physics Institute - A. A. Bogdanov, V. A. Kaplin, A. Karakash, S. B. Nurushev, M. F. Runtzo, M. N. Strikhanov

- State University of New York at Stony Brook - M. Rijssenbeek, C. Tang

- Tomsk Nuclear Physics institute- G. N. Dudkin, I. V. Glavanakov, Y. F. Krechetov, G. A. Naumenko, A. P. Potylitskin, G. M. Radutsky

- University di Genova and Sezione - M. Conte

- University Iowa - N. Akchurin, C. Newsom, Y. Onel

- University Padova - M. Pusterla

- University Texas - K. De, A. Vartapetian

- University di Trieste and Sezione-R. Giacomich, A. Penzo, P. Schiavon

This is an experiment to study proton-proton (pp) elastic scattering experiment at RHIC. Using both polarized and unpolarized beams, the experiment will study pp elastic scattering from $\sqrt{s}_{s}=60 \mathrm{GeV}$ to $\sqrt{s}=500 \mathrm{GeV}$ in two kinematical regions. 
Home Page http://www. phenix.bnl.gov

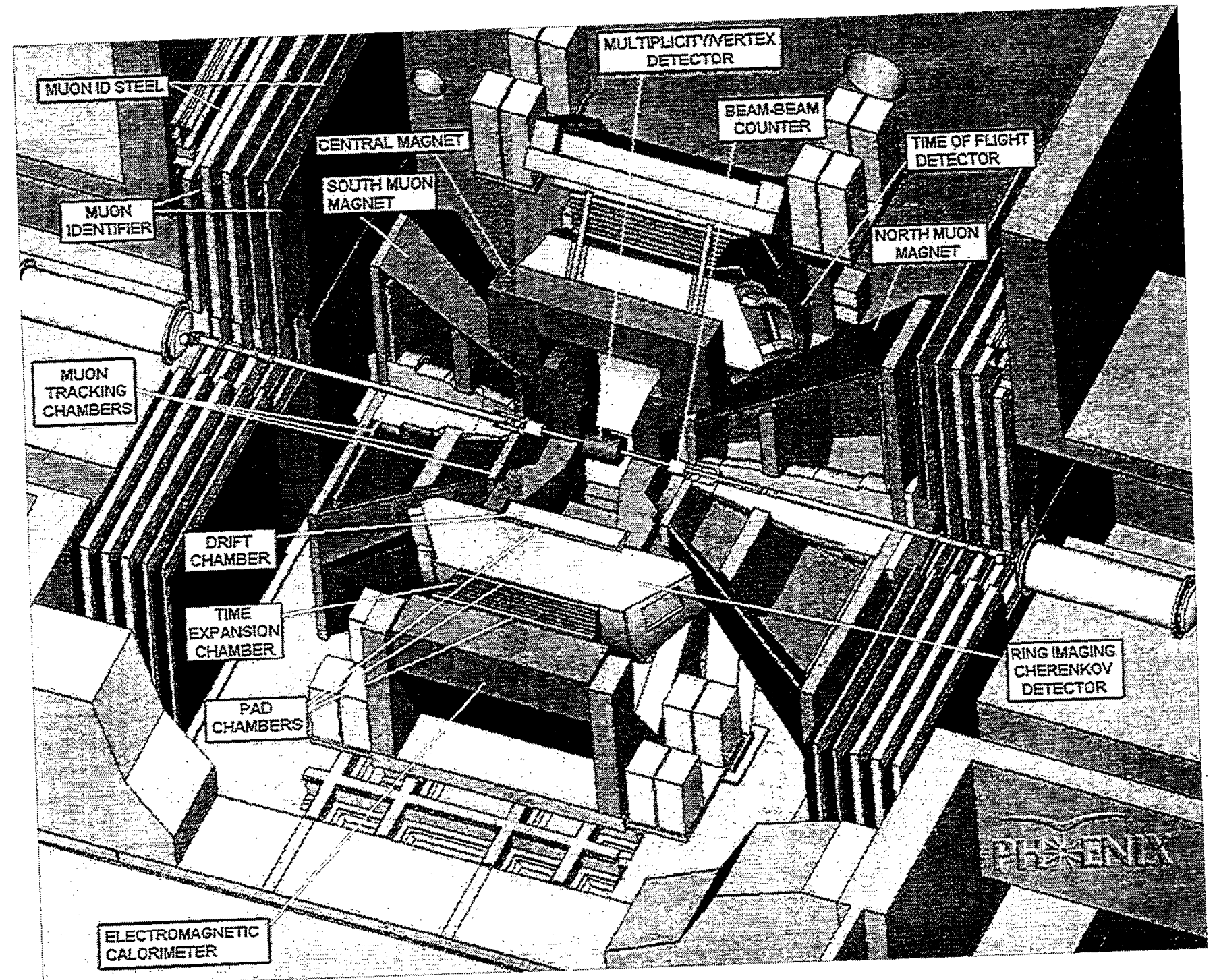


SPOKESMAN: W.A. ZAJC

The PHENIX collaboration consists of over 430 physicists and engineers from 43 participating institutions in 11 countries (Brazil, Canada, China, Germany, India, Israwl, Japan, Korea, Russia, Sweden and the U.S.A.) A comparable number of support personnel also work on PHENIX.

PHENIX is a very large detector system, which is designed to detect, identify, and measure the momentum of each of the many different kinds of particles produced at RHIC. PHENIX comprises three electromagnets, four instrumented spectrometers or arms, and inner detector systems.

PHENIX looks deep into the source of RHIC physics to learn about the earliest times of quark gluon plasma formation in heavy-ion collisions, and uncover the secrets of the spin structure of the proton in polarized proton collisions.

The design of the detectors and readout has been optimized across a very broad dynamic range from A-A collisions (low rate, large events, high occupancy) to $\overrightarrow{\mathrm{p}}-\overrightarrow{\mathrm{p}}$ collisions (high rate, small events, low occupany). This result of this optimization is a detector with the ability to measure both large cross section hadronic phenomena and rare processes. 


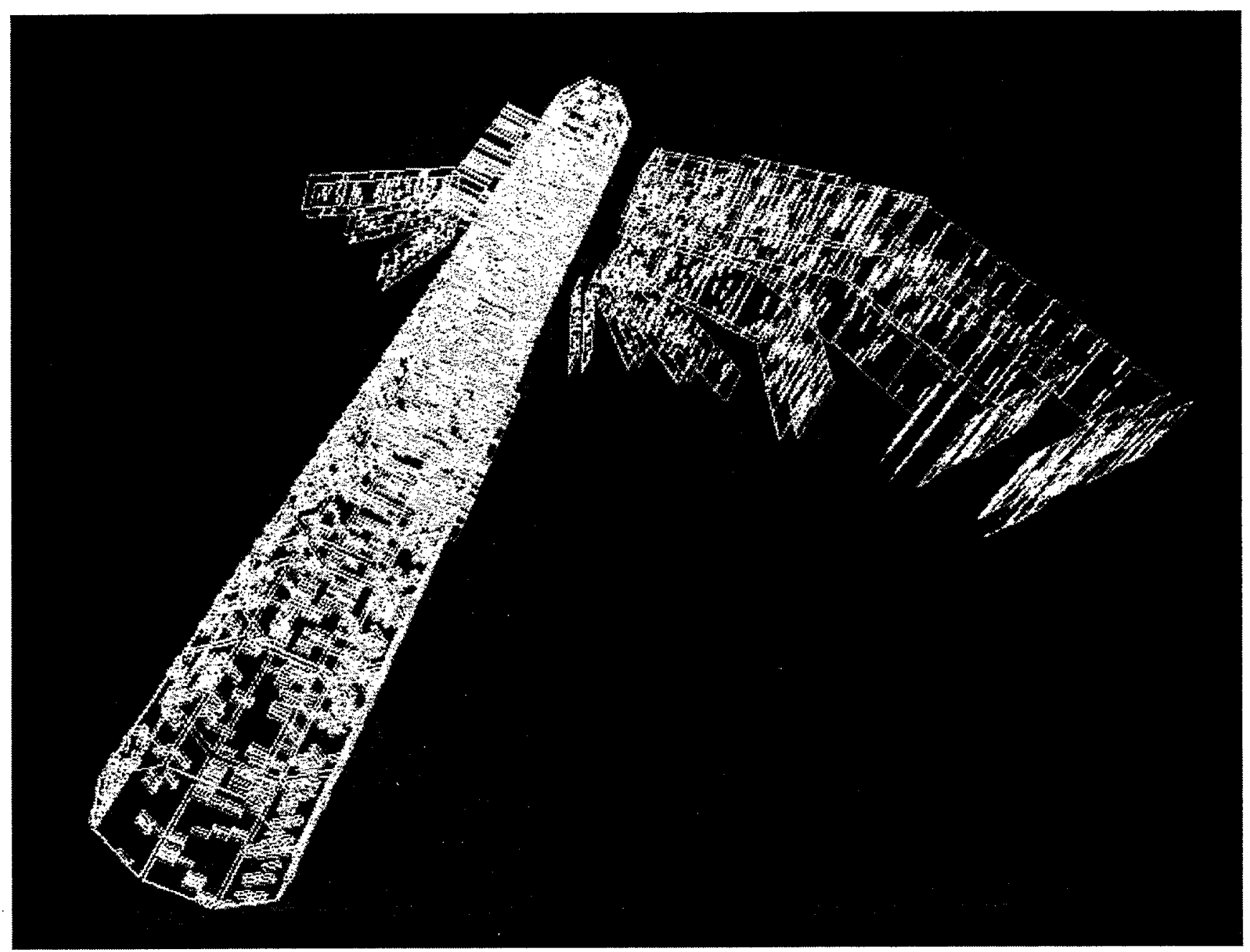

Gold-Gold Ion Collisions at PHOBOS

The figure shows the response of our Silicon Detectors to particles produced in collisions of two gold ions. The green points show where the particles hit the silicon. 
EXPERIMENT-

SPOKESMAN: W. BUSZA

The PHOBOS collaboration consists of over 100 physicists and engineers. A comparable number of support personnel also work on PHENLX.

- Argonne National Laboratory

- Brookhaven National Laboratory

- Institute of Nuclear Physics - Krakow, Poland

- Jagiellonian University - Krakow, Poland

- Massachusetts Inst. of Technology

- National Central University - Taiwan

- University of Rochester

- University of Illinois - Chicago

- University of Maryland

PHOBOS consists of many silicon detcctors surrounding the interacton region. With these detectors physicists will be able to count the total number of produced particles and study the angular distributions of all the products. With this array they will be on the look out for unusual events, fluctuations I the umber of particles and angular distribution. Physicists know from other branches of physics that a characteristic for phase transitions are fluctuations in physical observables. In order to obtain more detailed information about these events the PHOBOS detector has two high quality magnetic spectrometers which study, in detail, $1 \%$ of the produce particles.

The PHOBOS detector is able to measure quantities such as the temperature, suze, and density of the fireball produced in the collision. It studies the ratios of the various particles produced. With this information it should be possible to both detect and study a phase transition that might occur between Quark-Gluon Plasma (QGP) and ordinary nuclear matter. 


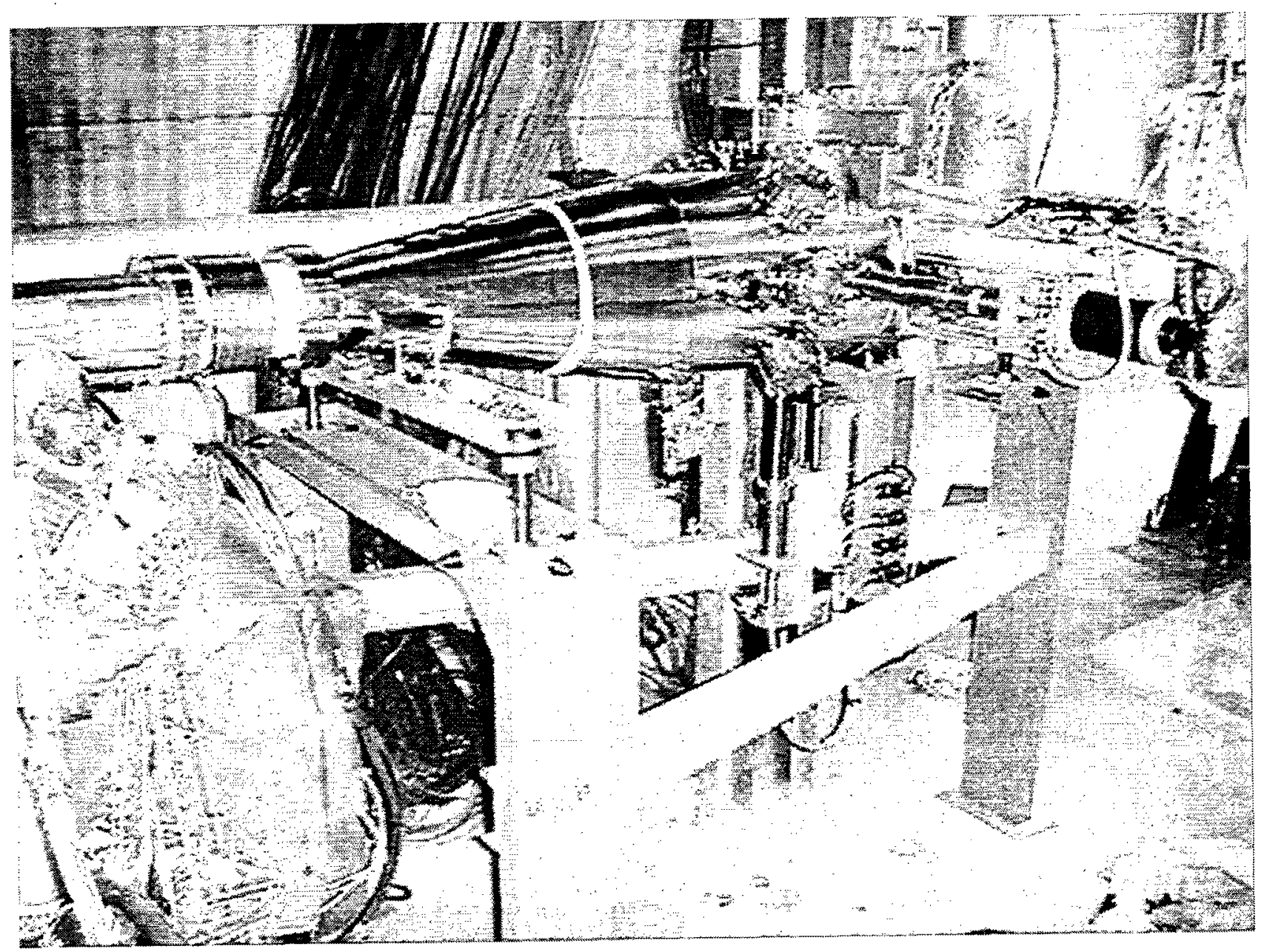

First Polarimeter Chamber Installed in Blue Ring 


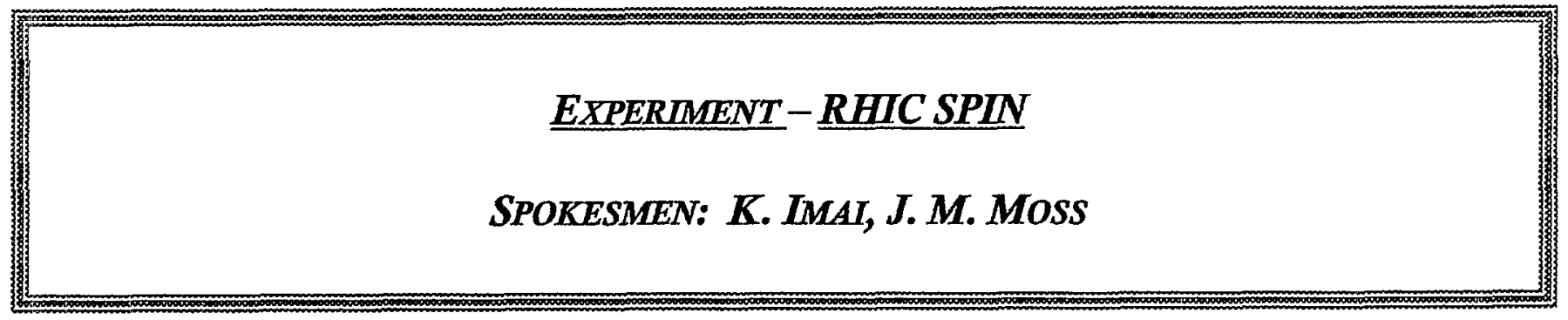

The RHIC SPIN collaboration consists of over 100 physicists and engineers. A comparable number of support personnel also work on PHENIX.

- Bombay Research Institute - India

- Brookhaven National Laboratory

- Chinese Instituate of Atomic Energy - Beijing

- Chung-ang University - Seoul, Korea

- Columbia University

- Institute of High Energy Physics - Protvino, Russia

- Institute of Modern Physics - Protvino, Russia

- Institute for Physical and Chemical Research - Wako, Japan

- Iowa State University

- Joint Inst. for Nuclear Research - Dubna, Russia

- Korea Úniversity

- Kurchatov Inst. of Atmoic Energy - Moscow, Russia

- Kyoto University - Japan

- Lawrenice Livermore Natl. Laboratory

- Los Alamos Natl. Laboratory

- Louisiana University

- New Mexico State University

- Oalk Ridge Natl. Laboratory

- Peking University

- Petersburg Nuclear Physics Institute - St. Petersburg, Russia

- Seoul National University

- Soong-Sil University - Seoul, Korea

- Tolkyo Institute of Technology

- University of California - Riverside

- University of Sao Paulo - Brazil

- University of Tenneseee

- Vanderbilt University

- Yonsei University - Seoul, Korea

The RIKEN BNL collaboration for Spin Physics fabricated and installed Siberian Snakes an dSpin Rotators in the RHIC main rings to accelerate polarized protons up to $250 \mathrm{GeV}$ and upgraded the PHENIX detector by installing new mouon arms to enhance the acceptance of single and di-muons. This making it feasible to study the polarized quark and anti-quark structure function by way of the Drell-Yan processes and vector boson productions, and investigate gluon polarization via heavy quark production and gluon Compton scattering. 


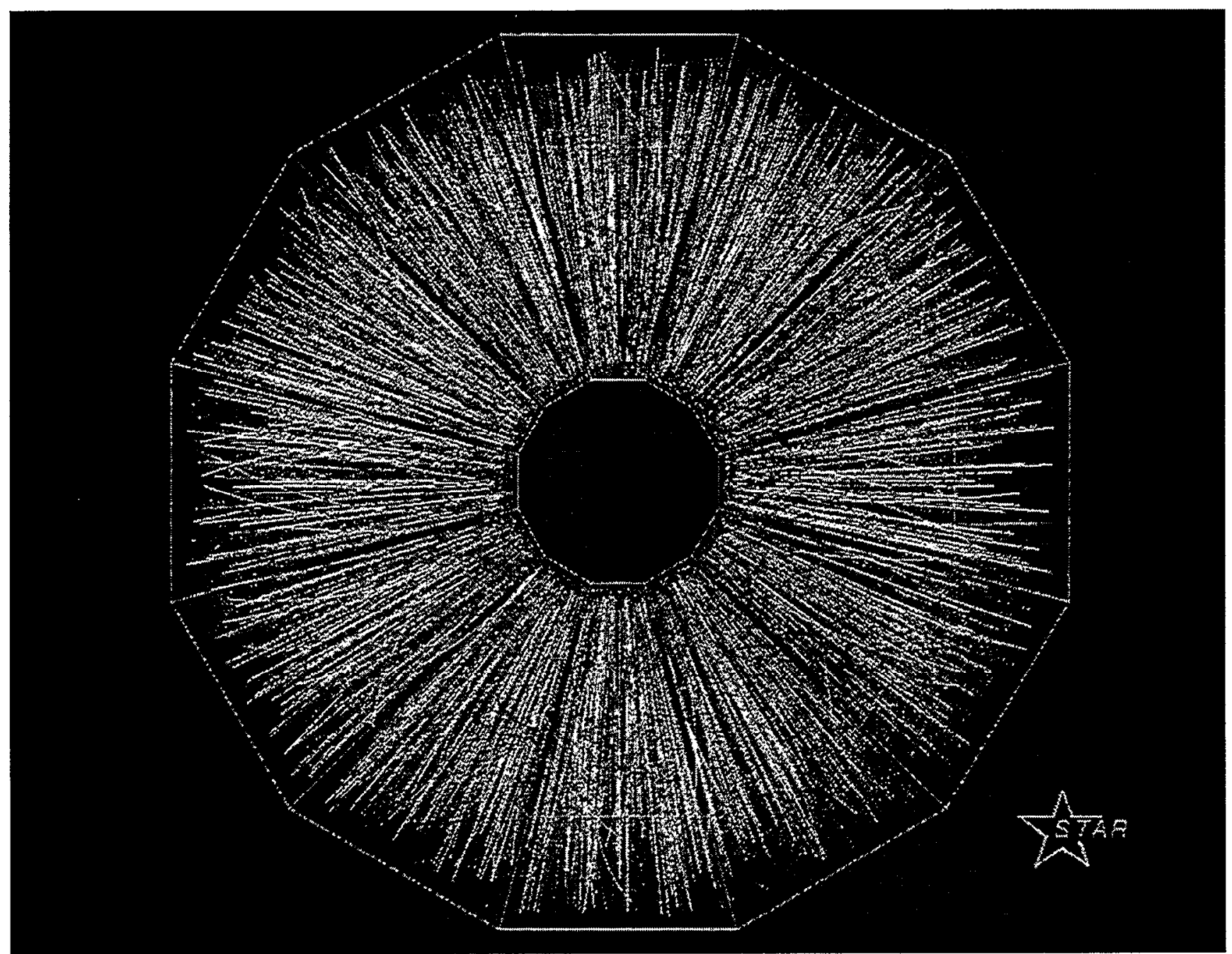

End view of a collision of two 30-billion-electron-volt gold beams in the STAR detctor at RHIC.

The beams travel in opposite directions at nearly the speed of light before colliding. 
The STAR collaboration consists of hundreds of physicists and engineers. A comparable number of support personnel also work on PHENIX.

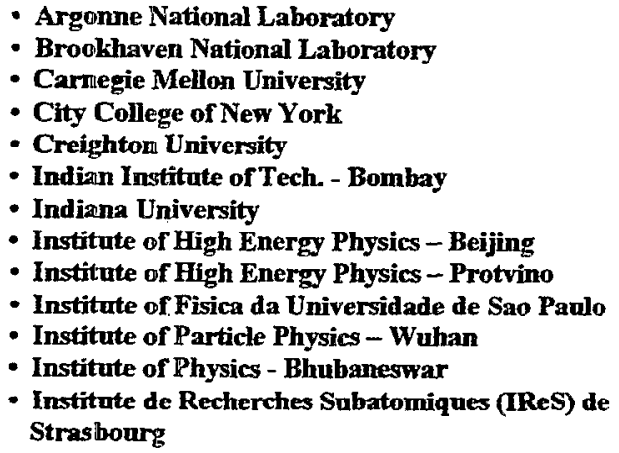

\author{
- Space Sciences Laboratory \\ - SUBATECH, Nantes \\ - Texas A\&M University \\ - University of Birmingham \\ - University of California-Davis \\ - University of California - Los Angeles \\ - University of Frankfurt \\ - University of Rajasthan \\ - University of Texas - Austin \\ - University of Washington \\ - Variable Energy Cyclotron Center - Calcutta \\ - Warsaw University of Technology \\ - Wayne State University \\ - Yale University
}

The Solenoidal Tracker At RHIC (STAR) experiment is designed to search for signatures of quark-gluon plasma (QGP) formation and to investigate the behavior of strongly interacting matter at high energy density. The experimental emphasis in the STAR heavy ion program is on the correlation of many observables on an event-by-event basis, and the hard scattering of partons as a penetrating probe of high density nuclear matter.

A second focus of the STAR experimental program is to study the spin-dependent parton distributions of the proton, using beams of transverse and longitudinally polarized protons. A specific goal is to measure the polarization of gluons in the proton, using the QCD "Compton" process (qg $\rightarrow \mathrm{q \gamma}$ ) as a probe.

The STAR detection system consists of a Time Projection Chamber (TPC) and a Silicon Vertex Tracker (SVT) inside a solenoidal magnet, enabling tracking, momentum analysis, particle identification by $\mathrm{dE} / \mathrm{dx}$, and location of primary and secondary vertices with full azimuthal coverage $(\Delta \Phi=2 \pi,|\eta|<1)$. A Central Trigger Barrel (CTB) of scintilllators surrounding the TPC, and Zero Degree Calorimeters (ZDCs) located at \pm 18 meters from the interaction point provide a collision geometry trigger, and the ability to selectively veto events according to the number of spectator neutrons going forward. A set of Forward Trigger Detectors (FTDs) designed to detect interactions by measuring the forward going charged particle multiplicity will be installed prior to the polarized proton rum in 2001 .

Forward Time Projection Chambers on either side of the interaction vertex provide additional tracking coverage in the acceptance $\Delta \Phi=$ $2 \pi, 2.5<|\eta|<4$. In addition, a Ring Imaging Cerenkov (RICH) detector covering approximately 1 square meter, and a 41 element patch TOF detector substituted for one of the CTB scintillators provide inclusive particle identification for high pt particles beyond the range where $\mathrm{dE} / \mathrm{dx}$ measurement in the STAR TPC is possible.

A Barrel Electromagnetic Calorimeter (BEMC) $(\Delta \phi=2 \pi,|\eta|<1)$ as well as an Endcap Electromagnetic Calorimeter $(E E M C)((\Delta \Phi=2 \pi$, $1<\eta<2$ ) on one cnd of the STAR detector will be used to measure neutral transvcrse energy, direct photon production, and jet cross sections. The detectors are under construction, with the installation of the first BEMC modules having begun. The installation of the first half of the EEMC is expected in the summer of 2002.

Additional detector components that will be added in 2002 include a Silicon Strip Detector (SSD) and a Photon Multiplicity Detector (PMD). The SSD will comprise a fourth layer of silicon tracking outside the existing three layers of silicon drift detectors which are part of the STAR SVT. It will be used to improve tracking efficiency and background rejection for secondary vertices from hyperon decays and to improve the standalone tracking capability of the silicon vertex tracker for low momentum particles. The Photon Multiplicity Detector is a fine-grained array of detector cells based on gaseous shower counting. It will be used to detect photon showers in the forward acceptance of STAR. 


\section{E787 DETECTOR}

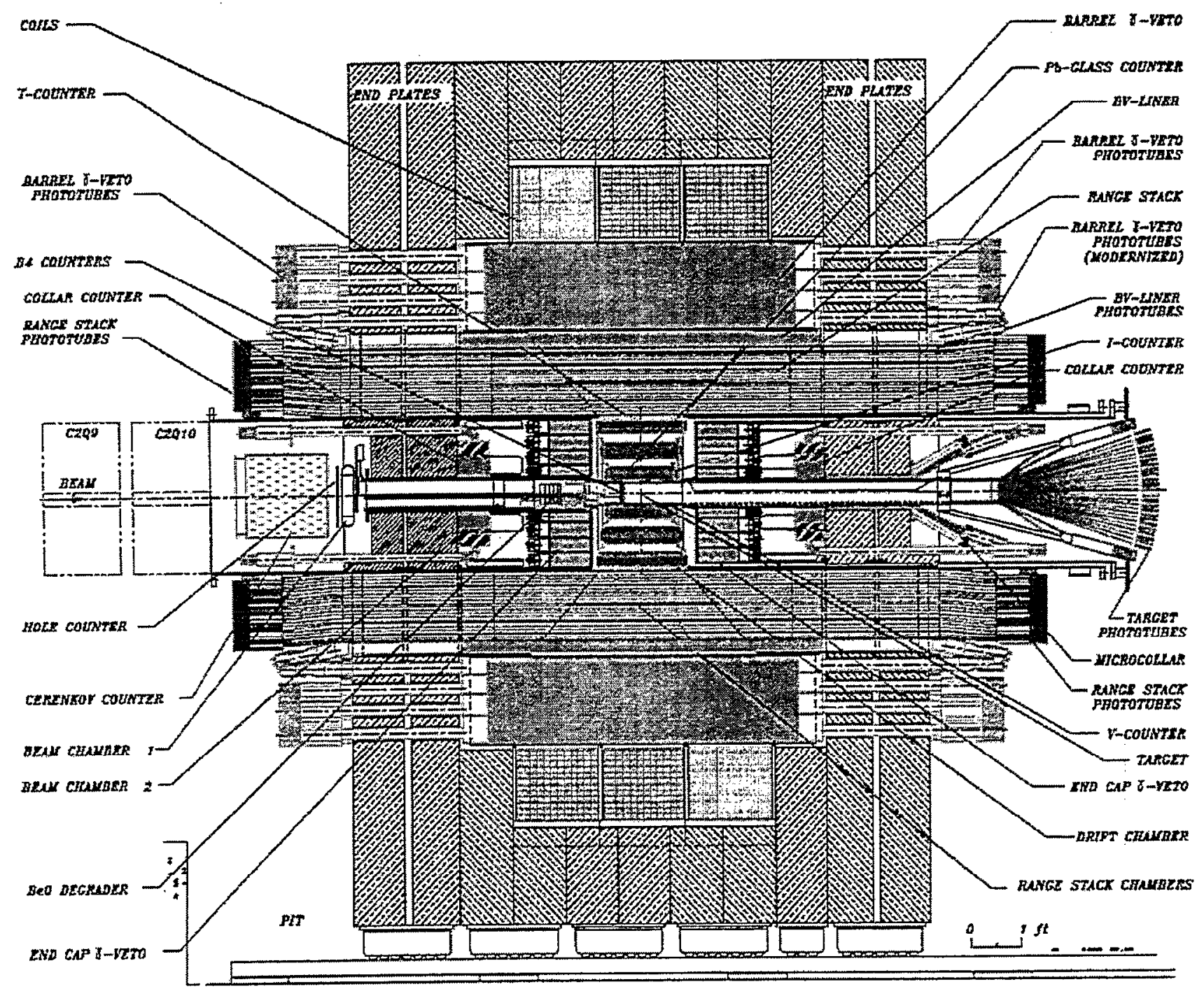




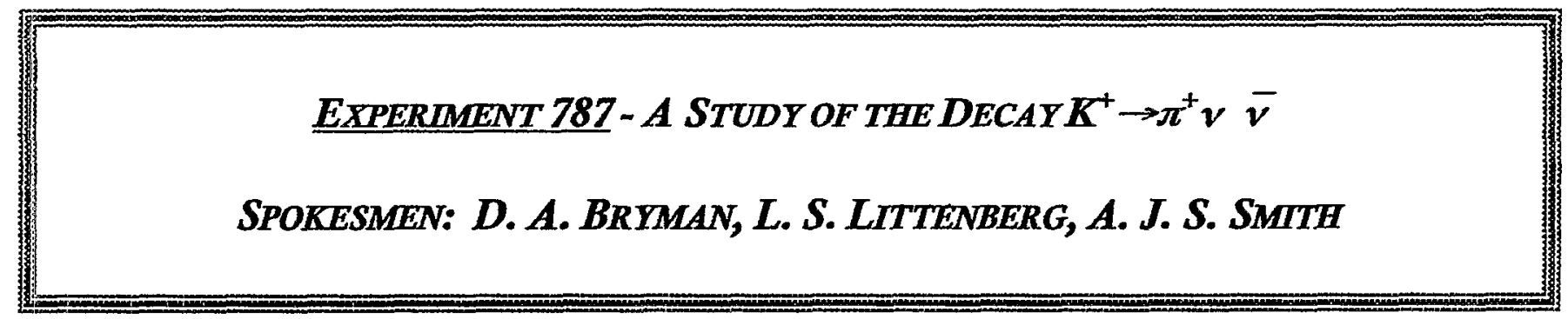

- Brookhaven National Laboratory

S. Adler, M. S. Atiya, I-H. Chiang, M. Diwan, J. S. Frank, J. S. Haggerty, V. Jain, S. Kettell, T. F.

Kycia, K. K. Li, L. S. Littenberg, C. F. Ng, R. C. Strand, C. Witzig

- Fukui University

M. Miyajima, Y. Tamagawa

- K]EK-National Laboratory for High Energy Physics

M. Aoki, T. Inagaki, S. Kabc, M. Kobayashi, T. K. Komatsubara, Y. Kuno, M. Kuriki, T. Morimoto, N. Muramatsu, H. Okuno, K. Omata, A. Otomo, T. Sato, T. Shinkawa, S. Sugimoto, K. Ukai, Y. Yoshimura

- Osaka University - T. Nakano

- Princeton University

A. Bazanko, P. D. Meyers, D. R. Relyea, F. C. Shoemaker, A. J. S. Smith, J. R. Stone

- TRIUIMF

P. Bergbusch, E. W. Blackmore, D. A. Bryman, S.Chen, A. Konaka, J. A. Macdonald, J. Mildenberger, T. Numao, J-M. Poutissou, R. Poutissou, G. Redlinger

- University of Alberta

P. Kitching, S. Ng, R. Soluk

Experiment 787 was a search for reactions of the type $\mathrm{K}^{+} \rightarrow \pi^{+} \mathrm{XX}$, where $\mathrm{X}$ is a weakly interacting light neutral particle. The expcriment was sensitive to such decays at the $10^{-10}$ level. The prime candidate was $\mathrm{K}^{+} \rightarrow \pi^{+} v \bar{v}$ which provided a uniquely stringent test of the Standard Model and its parameters because it is the only experimentally accessible process that is dominated by an unambiguously calculable higher order weak interaction. Two body decays $\mathrm{K}^{+} \rightarrow \pi^{+} \mathrm{X}$ were also searched for to place constraints on the existence of a variety of hypothetical particles such as axions, familons, hyperphotons and supersymmetric neutrals predicted by extensions to the SM. Several other rare decays including $\mathrm{K}^{+} \rightarrow \pi^{+} \gamma \gamma, \mathrm{K}^{+} \rightarrow \pi^{+} \mu^{+} \mu^{-}, \pi^{0} \rightarrow \boldsymbol{v} \bar{v}$, and $\pi^{0} \rightarrow \gamma$ XX were also investigated. The former two, $\mathrm{K}^{+} \rightarrow \pi^{+} \gamma \gamma$ and $\mathrm{K}^{+} \rightarrow \pi^{+} \mu^{+} \mu^{-}$, were discovered by this experiment. A candidate for $\mathrm{K}^{+} \rightarrow \pi^{+} v \bar{v}$ was observed by E787. 


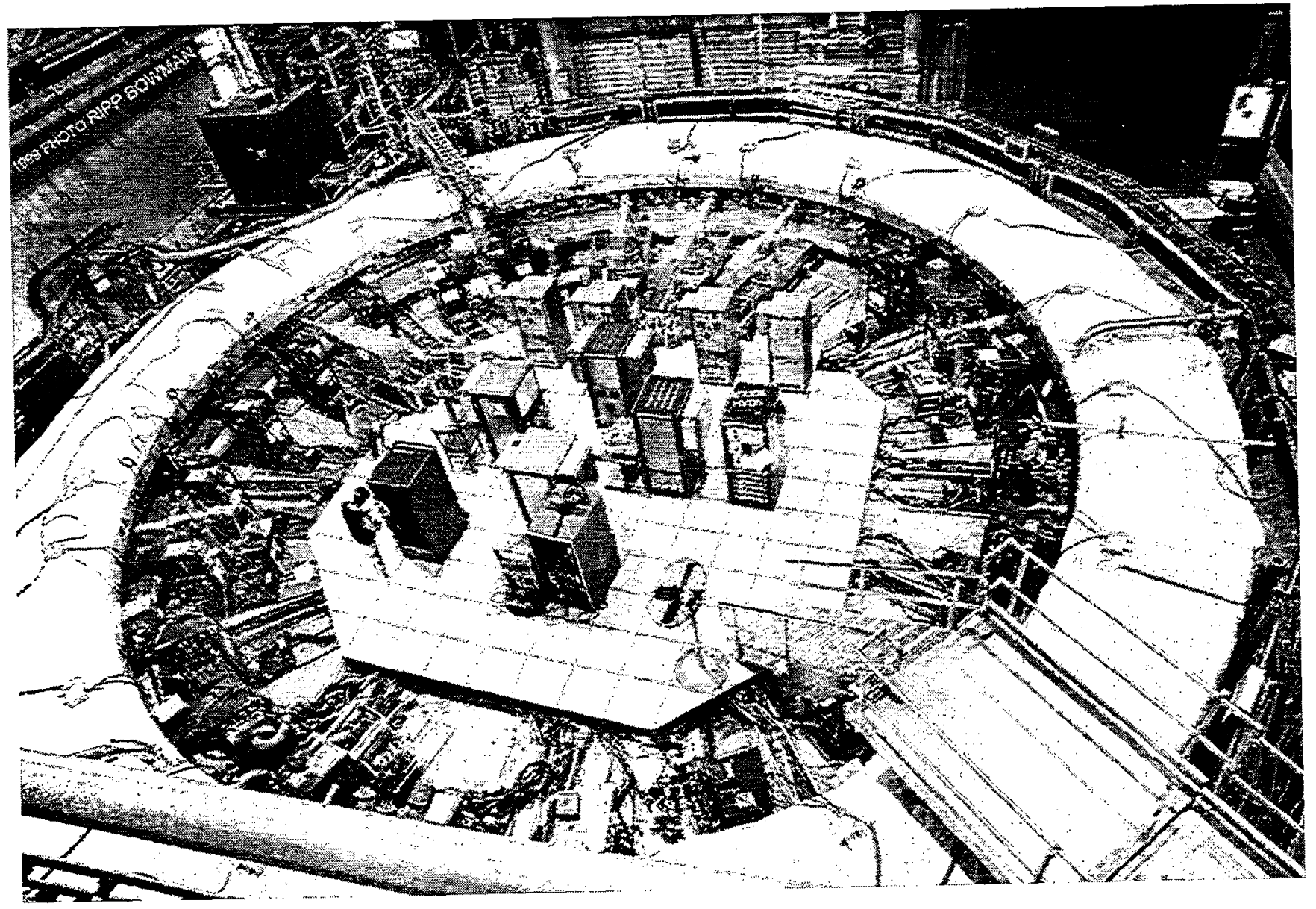




\title{
EXPERIMENT 821 - A NEW PRECTSION MEASUREMENT OF THE MUON G-2 AT THE LEVEL OF 0.35 PPM
}

\author{
SPOKESMEN: V.W. HUGHES, W. M. MORSE, B. L. ROBERTS
}

- Boston University - R.M. Carey, W. Earle, E. Efstathiadis, M. Hare, E.S. Hazen, F. Krienen, J.P. Miller, J. Pale, O. Rind, B.L. Roberts, L.R. Sulak, A. Trofimov

- Brookhaven National Laboratory - J. Benante, H.N. Brown, G. Bunce, G.T. Danby, R. Larsen, Y. Y. Lee, W. Meng, J. Mi, W.M. Morse, C. Ozben, C. Pai, R. Prigl, R. Sanders, Y. K. Semertzidis, L. Snydstrup, T. Tallerico, M. Tanaka, D. Warburton

- Budker Institue for Nuclear Physics - V.P. Druzhinin, G.V. Fedotovich, B.I. Khazin, I. Logashenko, N. Ryskulov, S. Serednyakov, Yu.M. Shatunov, E. Solodov

- Cornell University - Y. Orlov

- Fairfield University - D. Winn

- Max Planck Institut fur Physik - U. Haeberlen

- National Laboratory for High Energy Physics (KEK) - A.Yamamoto

- Tokyo Institute of Technology - M. Iwasaki, M. Kawamura

- Yale University - H. Deng, S.K. Dhawan, F.J.M. Farley, M. Grosse-Perkekamp, V.W. Hughes, D. Kawall, W. Liu, J. Pretz, S. I. Redin, A. Steinmetz

- University of Heidelberg - A. Grossmann, K. Jungmann, D. von.Walter, G. zu Putlitz

- University of Illinois - P. Debevec, W. Deninger, F. Gray, D.W. Hertzog, C. J. G. Onderwater, C. Polly, S. Sedykh, M. Sossong, D. Urner

- University of Minnesota - P. Cushman, L. Duong, S. Giron, J. Kindem, I. Kronkvist, R. McNabb, D. Miller, C. Timmermans, D. Zimmerman

The anomalous gyromagnetic ratio of the muon $(\mathrm{g}-2)$ will be measured to $0.35 \mathrm{ppm}$ or a factor of 20 times better than it is currently known. The predicted contribution to (g-2) from the first and second order $\mathrm{W}^{ \pm}$and $Z^{0}$ radiative corrections is predicted to be $1.3 \mathrm{ppm}$, so this experiment will provide a direct test of the electroweak radiative corrections, and hence of the renormalizability of the Glashow-Weinberg-Salam theory. Since $a_{\mu}$ is sensitive to a wide range of non-standard model effects there is a window in which to search for new physics. W or muon substructure, super-symmetry and the existence of new gauge bosons are several possibilities. A $14 \mathrm{~m}$ diameter superferric muon storage ring is now operational. Data collection began in FY 1997. 


\section{Polarized Proton Experiments in the AGS with a Partial Siberian Snake (E880)}

(ANL,BNL,INDIANA,TRIUMF,KEK,RIKEN,IHEP)

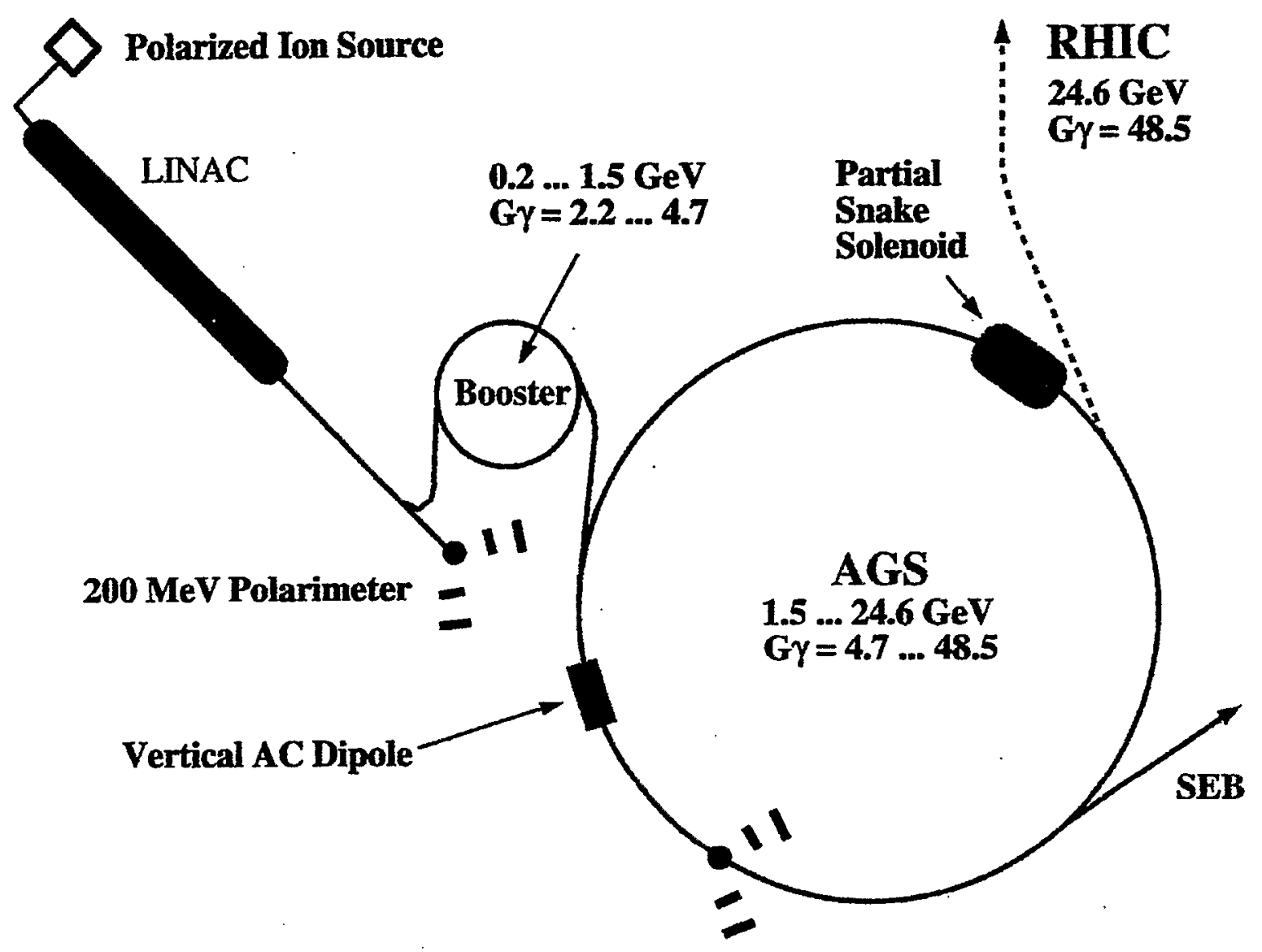

AGS Internal Polarimeter 
Beam: AGS Internal Beam Status: Completed Hours Charged/Approved: 729/320

\title{
EXPERIMENT 880 - THE EFFECTS OF A PARTTAL StBERTAN SNAKE ON POLARIZATTON AT THE AGS
}

\author{
SPOKESMEN: S. Y. LEE AND T. ROSER
}

- Argonne National Laboratory - C. Allgower, M. Bai, ${ }^{(1)}$ K. Krueger, H. Spinka, L. Teng, D. G. Underwood, A. Yokosawa

- Brookhaven National Laboratory - L. Ahrens, J. Alessi, K. Brown, G. Bunce, P. Cameron, E. D. Courant, J. W. Glenn, H. Huang, P. Ingrassia, Y. Y. Lee, A. Luccio, Y. I. Makdisi, L. Ratner, K. Reece, T. Roser, J. F. Skelly, A. Soukas, M. Syphers, S. Tepikian, R. E. Thern, N. Tsoupas, W. van Asselt, N. Williams

- Fermilab - V. Bharadwaj, S. Hseuh

- Indiana University IUCF - P. Chu, D. Jeon, S.Y. Lee

- Institute for High Energy Physics, Serpukhov - A. Ufimtsev

- KEK - S. Hiramatsu, Y. Mori, H. Sato, K. Yokoya

- Fiken - T. Katayama, M. Okamura, T. Tominaka

- TRIUMF - U. Wienands ${ }^{(2)}$

This group has built a 4.7 Tesla-meter room temperature solenoid which was installed in a 10-foot long AGS straight section. AGS polarized proton beam time was used to perform partial snake experiments. These experiments tested successfully the idea of using a partial snake to correct all de-polarizing imperfection resonances. Intrinsic depolarizing resonances are successfully overcome by using an AC vertical dipole to generate coherent betatron oscillation.

(1) Also at Indiana University

(2) Present address: SLAC 


\section{BNL-AGS E896 EXPERIMENTAL LAYOUT}

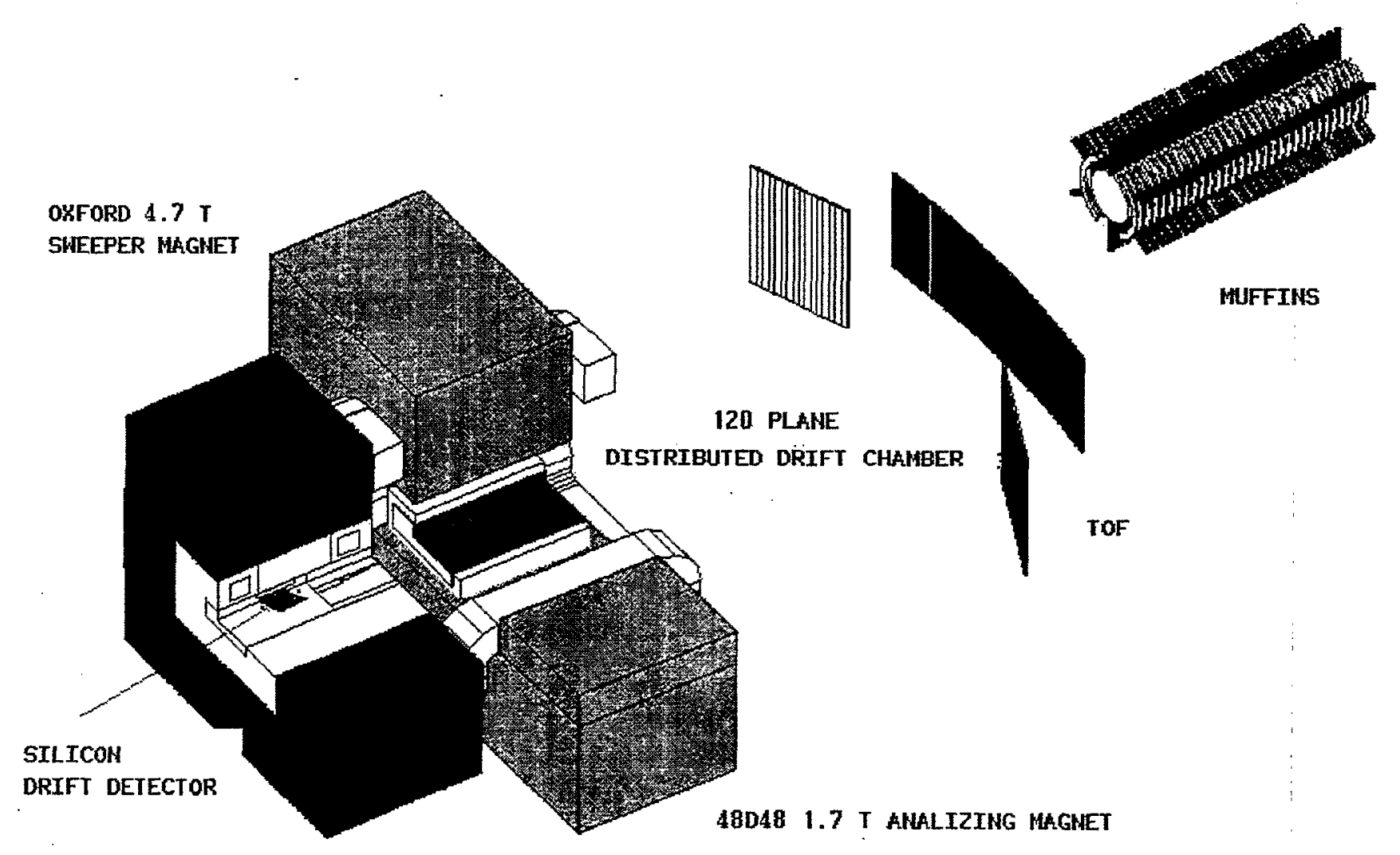




\section{EXPERTMENT 896-A PROPOSAL TO THE BNL AGS: \\ To Search for a Short-Lived $H_{o}$ DiBaryon, Short-Lived Strange Matter, and to Investigate Hyperon Production in 11.6 A GeV/c AuAu Collision}

\section{SPOKESMEN: H. CRAWFORD AND T. HALLMAN}

- Brookhaven National Laboratory - W. Christie, R. Debbe, T. Hallman, T. Ljubicic, R. Longacre, D. Lynn, J. Mitchell, E. Mogavero, A. Saulys

- Carnegie-Mellon University - C. Brown, M. Kaplan, Z. Milosevich, D. Russ

- CERN - P. Sonderegger

- Johns Hopkins University - L. Madansky

- Lawrence Berkeley Laboratory - L. Greiner, P. Lindstrom, J. Marx, I. Sakrejda

- NASA-Goddard Space Flight Center - J. W. Mitchell

- Ohio State University - T. Humanic, I. Kotov, G. LoCurto, E. Sugarbaker

- Rice University - B. Bonner, K. Kainz, W. Llope, G. Mutchler, E. Platner, P. Yepes

- Space Science Laboratory - M. Bennctt, H. Crawford, M. Cronqvist, I. Engclage, M. Flores, L. Greiner, E. Judd, G.Visser

- Wayne State University - R. Bellwied, M. Nachaet, S. Nehmeh, S. Pandey, J. Sheen, J. Takahashi, K. Wilson

- Yale University - G. Kunde, S. Kumar, F. Rotondo, N. Smirnoff

- University of California-Los Angeles - H. Huang, G. Igo, S. Kelly, S. Trentalage

- University of Catania - S. Albergo, D. Boemi, Z. Caccia, S. Costa, A. Insolia, C. Nociforo, R. Potenza, G. Russo, A. Tricoma, C. Tuve

- University of Michigan - R. Welsh

- University of Texas-Austin - G. Hoffman, P. Jensen, S. Paganis, P. Riley, J. Schambach, J. Tang

The experiment is a search for the $\mathrm{H}^{\circ}$ dibaryon and for new states of nuclear matter produced in nucleus-nucleus (AuAu) collisions at the AGS. The experiment enhances the existing AA program by extending the search into regions of shorter lifetime and complements the existing double-strangeness-exchange program by offering access to a new, more probable doorway channel, the coalescence of two $\Lambda$ 's into a bound di- $\Lambda$. The detector is a capable of unambiguously identifying the topological signature of unstable particle decays as well as the rigidity of each particle produced, affording a sensitive search for new metastable states and investigation of the properties of known strange particles such as the $\Lambda$ polarization and the $\Lambda \Lambda$ potential. 


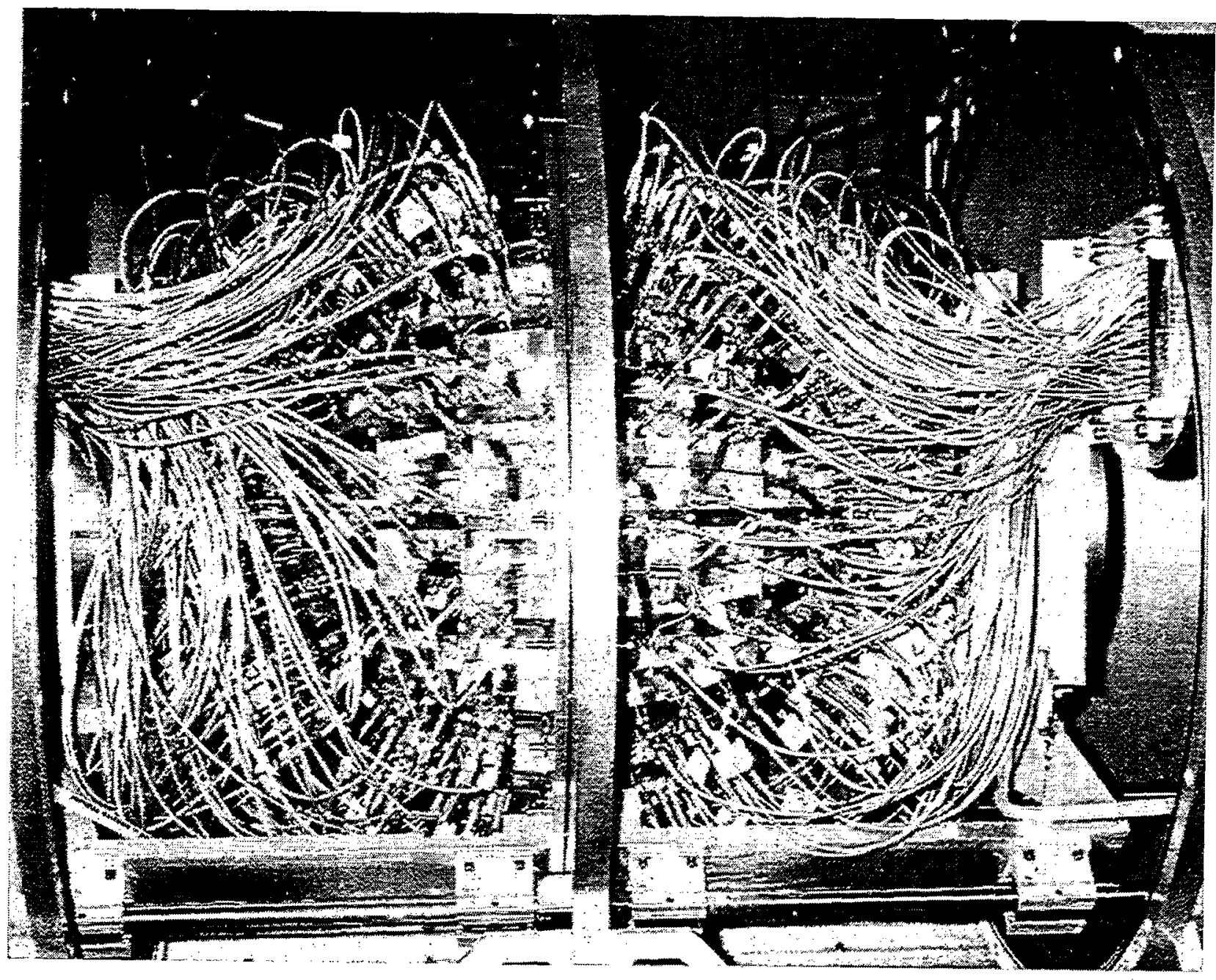

Full View of the ISiS Detector 


\section{EXPERTMENT 900 - ENERGY DISSIPATION AND MULTIFRAGMENTATTON IN H + A REACTIONS BETWEEN 2 AND 24 GEV/C}

\section{SPOKESMEN: K. KWLATKOWSKI AND V. E. VIOLA}

- Argonne National Laboratory - B. Back

- Brookhaven National Laboratory - S. Gushue, L. P. Remsberg

- Indiana University - W-Ch. Hsi, K. Kwiatkowski, T. Lefort, V. E. Viola, N. R. Yoder

- Los Alamos National Laboratory - D. S. Bracken, K. B. Morley

- Simon Fraser University - R. Korteling

- Texas A\&M University - F. Gimeno-Nogues, E. Ramakrishnan, D. Rowland, S. J. Yennello

- Warsaw University - L. Pienkowski

- University of Maryland - H. Breuer

This experiment had exclusive studies of target fragmentation in $2-24 \mathrm{GeV} / \mathrm{c}$ hadron $(\rho, \bar{\rho}$ and $\pi)$-induced reactions. Measurements were performed with the Indiana Silicon Sphere $4 \pi$ detector array, capable of identifying $\mathrm{H}$ and $\mathrm{He}$ isotopes and $\mathrm{Z}=3-20$ fragments for target rapidity ejectiles over a wide dynamic range. The primary physics objectives were twofold: (1) to improve the understanding of energy dissipation phenomena for central collisions in the $h+A$ reaction at relativistic energies, and (2) to examine the decay modes of hot nuclear matter excited by simple hadron probes. The bombarding energy regime was chosen to overlap the region in which previous inclusive measurements at AGS had been interpreted in terms of a liquidgas phase transition in hot finite nuclei. It is in this energy region that the excitation of $\Delta, N^{*}$ and higher resonances provide an effective means of dissipating projectile energy into internal excitation energy of the target nucleus. Thus, these data placed fresh constraints on the new generation of transport codes, as well as current models of mulifragmentation. During the 1996 proton cycle, studies were successfully completed on the $p+{ }^{197} \mathrm{Au}$ system at $6.0,10.0,12.0$ and $14.6 \mathrm{GeV} / \mathrm{c}$ and $\pi^{-}+{ }^{197} \mathrm{Au}$ at 5.0, 9.2 and $11.0 \mathrm{GeV} / \mathrm{c}$. These measurements demonstrated an independence of energy deposition on projectile momentum and type for these reactions. In 1998 it measured the $7 \mathrm{GeV} / \mathrm{c} \bar{\rho}+{ }^{197} \mathrm{Au}$ reaction to search for enhanced energy deposition with antiproton beams. 


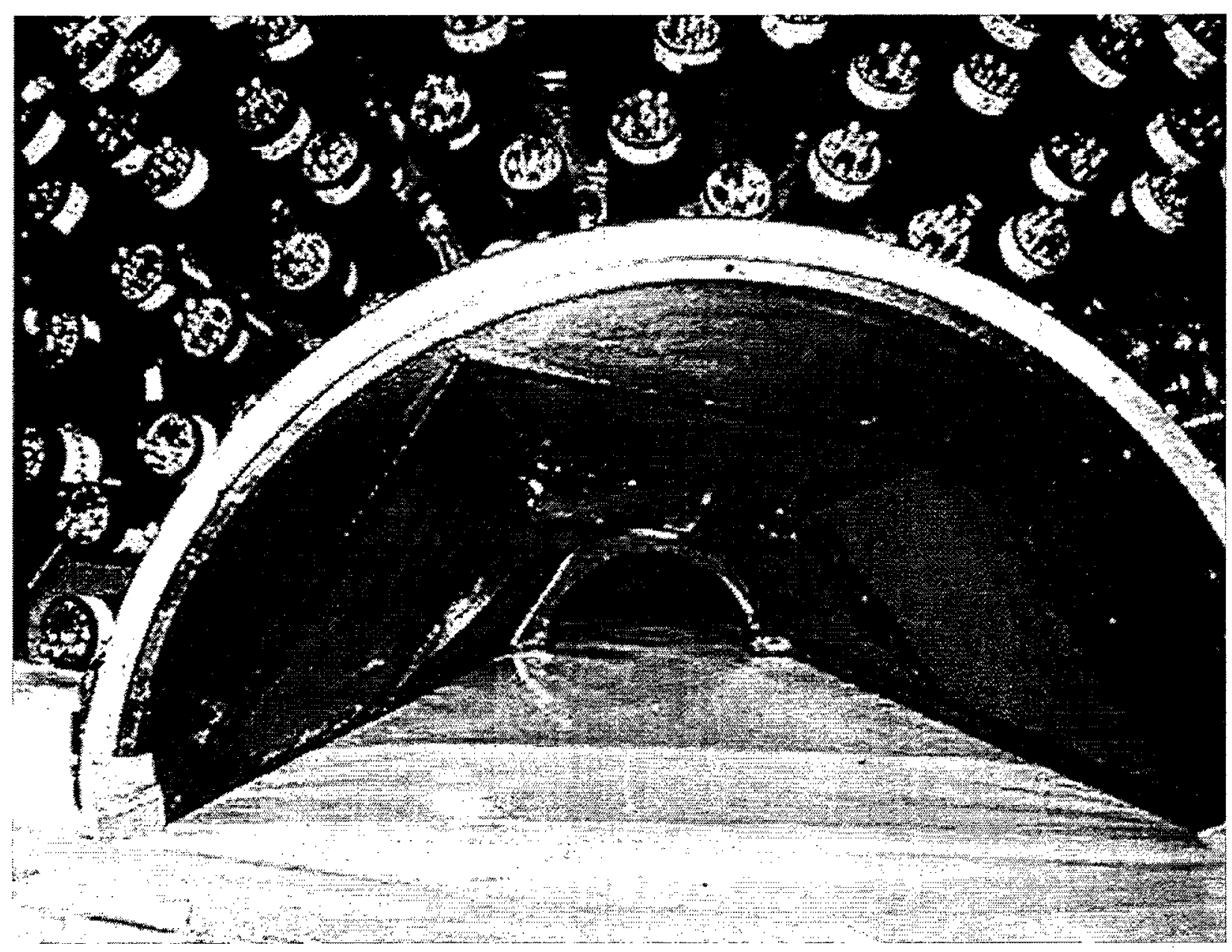




\section{EXPERIMENT 913 - BARYON SPECTROSCOPY WITH THE CRYSTAL BALL}

\section{SPOKESMAN: M. E. SADLER, H. SPINKA, W. B. TIPPENS}

- Abilene Christian University - R. Bagga, B. Draper, J. Huddleston, D. Isenhower, Z. Mulkey, M. Sadler

- Argonne National Laboratory - T. Kasprzyk, H. Spinka

- Arizona State University - J. Comfort, K. Craig, A. Ramirez

- Brookhaven National Laboratory - T. Kycia

- George Washington University - W. J. Briscoe, A. Shafi

- Kent State University - D. M. Manley

- Petersburg Nuclear Physics Institute-Gatchina - V. Abaev, V. Bekrenev, S. Kruglov, A. Kulbardis, I. Lopatin, A. Starostin

- Rudjer Boskovic Institute -I. Šlaus, I. Supek

- Valparaiso University - A. Gibson, D. Grosnick, D.D. Koetke, R. Manweiler, P. Nord, S. Stanislaus

- University of California-Los Angeles - M. Clajus, S. McDonald, A. Marusic, B.M.K. Nefkens, M. Pulver, W. B. Tippens

- University of Colorado - J. Patterson, J. Peterson

- Universit at Karlsruhe - H. Staudenmaier

- University of Regina - N. Knecht, G. Lolos, Z. Papandreou

This was a comprehensive experimental program in baryon spectroscopy using the SLAC Crystal Ball detector to make precision measurements of total and differential cross sections for neutral final states in $\pi p$ interactions using pion beams in the momentum range $0.4-1.9 \mathrm{GeV} / \mathrm{c}$. The angular distributions of all the neutrall final states such as $\gamma \mathrm{n}, \pi^{\circ}$ were measured simultaneously. The purpose was to improve the mass, width, and neutral branching fractions for the $\mathrm{N}^{*}$ resonances in this energy region. The Crystal Ball detector is a nearly $4 \pi$ multiphoton spectrometer, which is used to analyze events by reconstructing the invariant mass and, in conjunction with the measured beam momentum, the missing mass of the produced $\gamma$ rays. The Crystal Ball was located in the $\mathrm{C} 6$ beam line during this phase of the experiment. The beam momentum was limited to $\leq 750 \mathrm{MeV} / \mathrm{c}$. 
$1-40+$

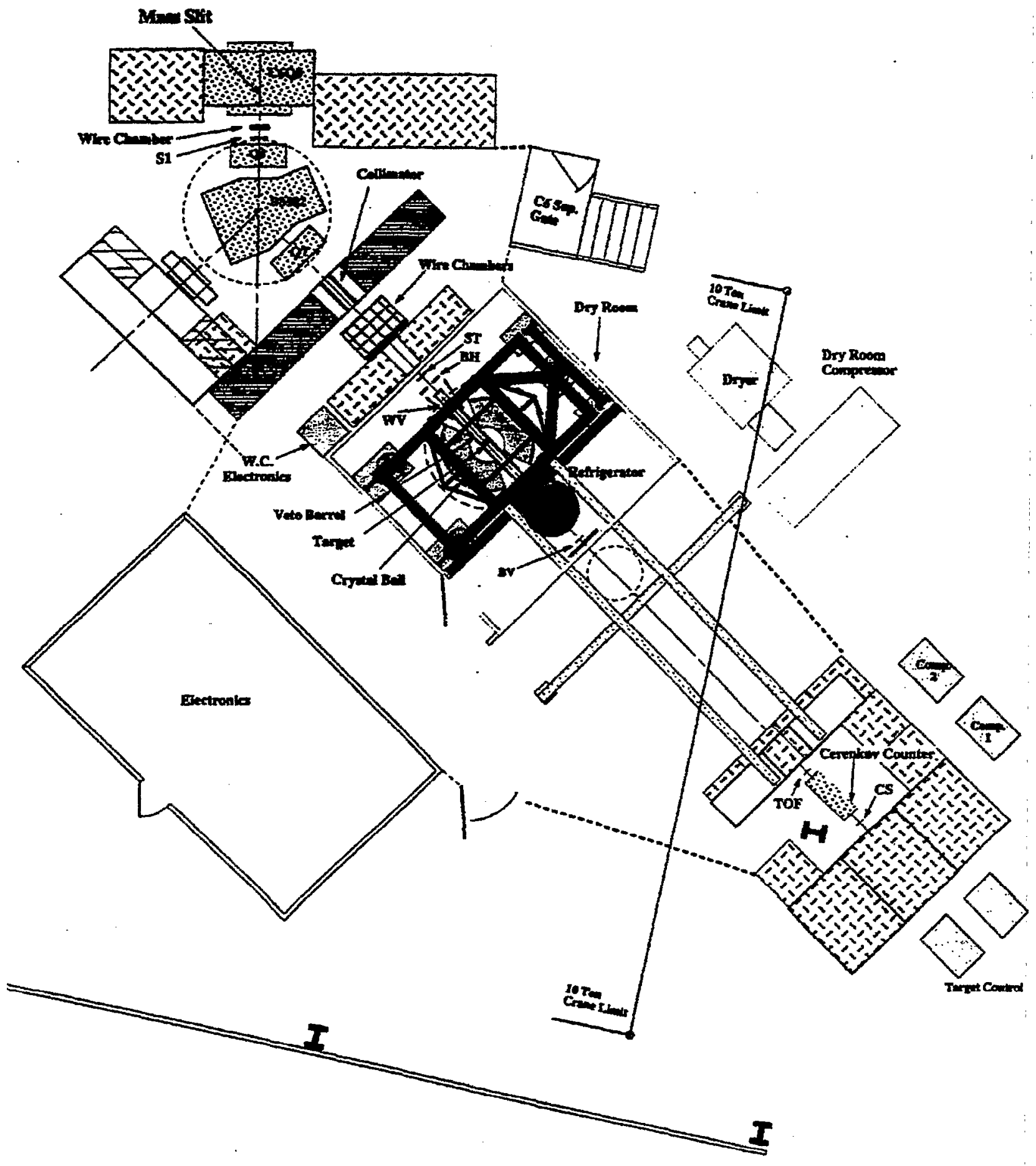




\section{EXPERIMENT 914 - NEUTRAL HYPERON SPECTROSCOPY \\ SPOKESMAN: B.M.K. NEFKENS, T. KYCLA, S. P. KRUGLOV}

- Abilene Christian University - R. Bagga, B. Draper, J. Huddleston, D. Isenhower, Z. Mulkey, M. Sadler

- Argonne National Laboratory - T. Kasprzyk, H. Spinka

- Arizona State University - J. Comfort, K. Craig. A Ramirez

- Brookhaven National Laboratory - T. Kycia

- George Washington University - W. J. Briscoe, A. Shafi

- Kent State University - D. M. Manley

- Petersburg Nuclear Physics Institute-Gatchina - V. Abaev, V. Bekrenev, S. Kruglov, A. Kulbardis, I. Lopatin, A. Starostin

- Rudjer Boskovic Institute -I. Šlaus, I. Supek

- Valparaiso University - A. Gibson, D. Grosnick, D.D. Koetke, R. Manweiler, P. Nord, S. Stanislaus

- University of California-Los Angeles - M. Clajus, S. McDonald, A. Marusic, B.M.K. Nefkens, M. Pulver, W. B. Tippens

- University of Colorado - J. Patterson, J. Peterson

- Universit at Karlsruhe - H. Staudenmaier

- University of Regina - N. Knecht, G. Lolos, Z. Papandreou

This experiment investigated the spectrum of $\Lambda^{*}$ and $\Sigma^{*}$ resonances via their neutral decays in the reactions:

$$
\begin{array}{lc}
\mathrm{K}^{-} \mathrm{P} \rightarrow \Lambda \gamma & 600-1800 \mathrm{MeV} / \mathrm{c}\left(\mathrm{p}_{\mathrm{lab}}\right) \\
\mathrm{K}^{-} \mathrm{P} \rightarrow \Lambda \pi^{0} & " \\
\mathrm{~K}^{-} \mathrm{P} \rightarrow \Lambda 2 \pi^{0} & 720-1800 \mathrm{MeV} / \mathrm{c}\left(\mathrm{p}_{\mathrm{lab}}\right) \\
\mathrm{K}^{-} \mathrm{P} \rightarrow \Lambda \eta & 600-1800 \mathrm{MeV} / \mathrm{c}\left(\mathrm{p}_{\mathrm{lab}}\right) \\
\mathrm{K}^{-} \mathrm{P} \rightarrow \Sigma^{0} \gamma & " \\
\mathrm{~K}^{-} \mathrm{P} \rightarrow \Sigma^{0} \pi^{0} & " \\
\mathrm{~K}^{-} \mathrm{P} \rightarrow \Sigma^{0} 2 \pi^{0} & \\
\mathrm{~K}^{-} \mathrm{P} \rightarrow \Sigma^{0} \eta & 890-1800 \mathrm{MeV} / \mathrm{c}\left(\mathrm{p}_{\mathrm{lab}}\right)
\end{array}
$$

Measurements of total and differential cross sections of these reactions were made simultaneously over the full angular range, using the Crystal Ball multi photon spectrometer and a LH2 target. The Crystal Ball has a $94 \%$ solid angle coverage and good energy and angular resolution. This phase of the experiment was conducted in the $\mathrm{C} 6$ beam line with a maximum momentum of $750 \mathrm{MeV} / \mathrm{c}$. 


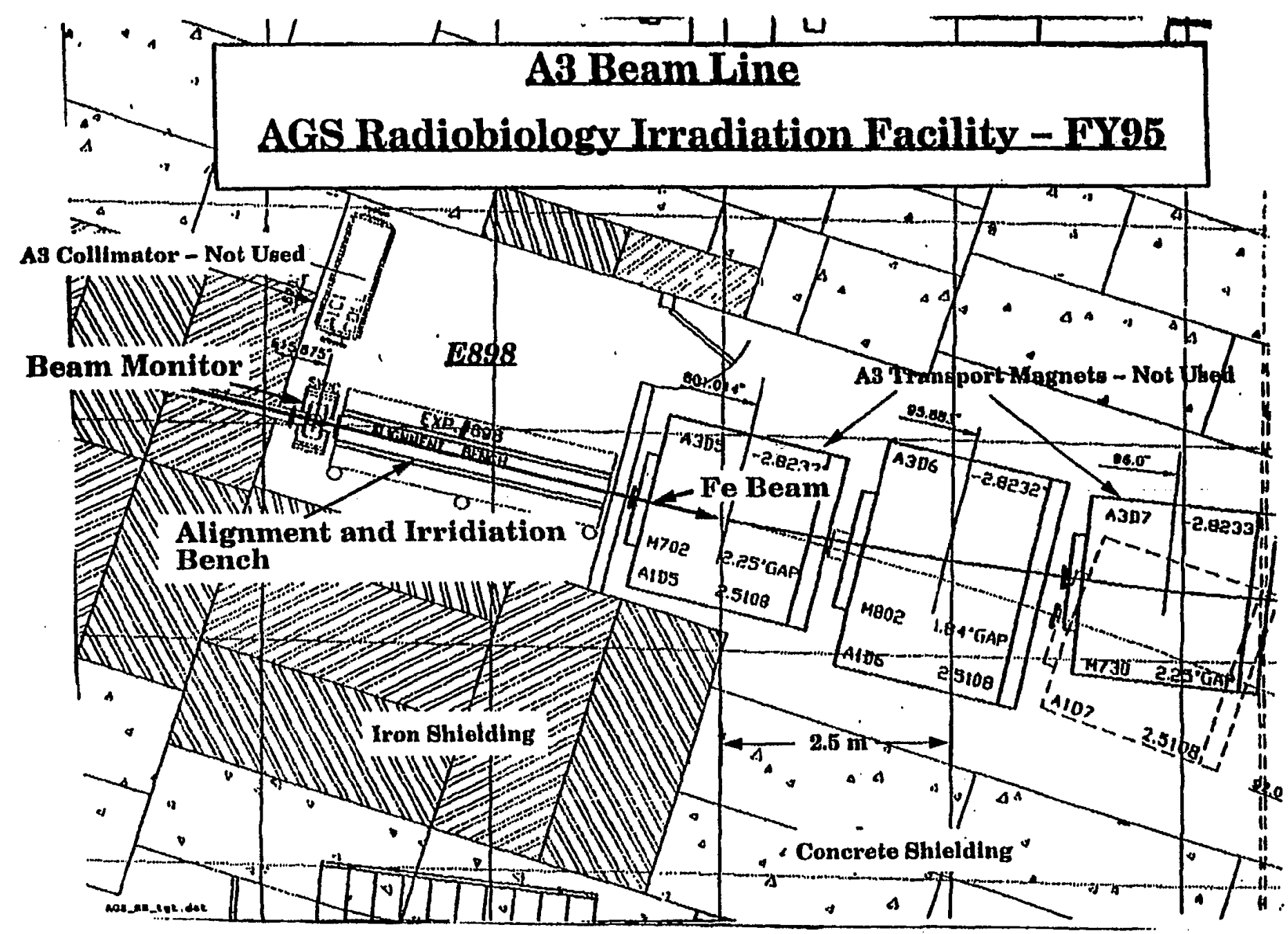




\section{EXPERMENT 919 - A STUDY OF THE GENETTC AND EPIGENETTC EFFECTS PRODUCED BY HIGH ENERGY HEAVY IONS}

SPOKESMEN: M. VAZQUEZ

- Alabama Agr. and Mechanical University - P. Kale

- Brookhaven National Laboratory - J. Bullis, J. Gatley, B. Sutherland, M. Vazquez

- Case Western Reserve University - H. Evans

- Colorado State University - T. Borak, G. Mariano, C. A. Waldren

- Columbia University - T. K. Hei

- Georgetown University medical Center - T. Jorgensen

- Lawrence Berkeley National Laboratory - P. Cooper, A. Kronenberg, J. Miller

- Loma Linda University - G. Nelson

- Los Alamos National Laboratory - D. J. Chen

- NASA Johnson Space Center - T. C-h Yang

- Natl. Inst. Of Radiological Sciences, Japan - Y. Furusawa

- Pacific Northwest National Laboratory - N. F. Metting

- USAF Armstrong Laboratory - A. Cox

- Washington State University - A. Brooks

- University of California - L. H. Lutze-Mann, W. Morgan

- University of Maryland Baltimore - E. Balcer-Kubiczek, B. Rabin

- University of Texas - M. Natarajan

This was a NASA effort in the AGS to study the genetic and epigentic effects produced by high-energy heavy ions. There were two runs labeled BNL-3, 4 for the period of October 1, 1996 to September 31, 1998. Each run provided approximately 150 hours of $1 \mathrm{GeV} /$ nucleon Fe ions. 


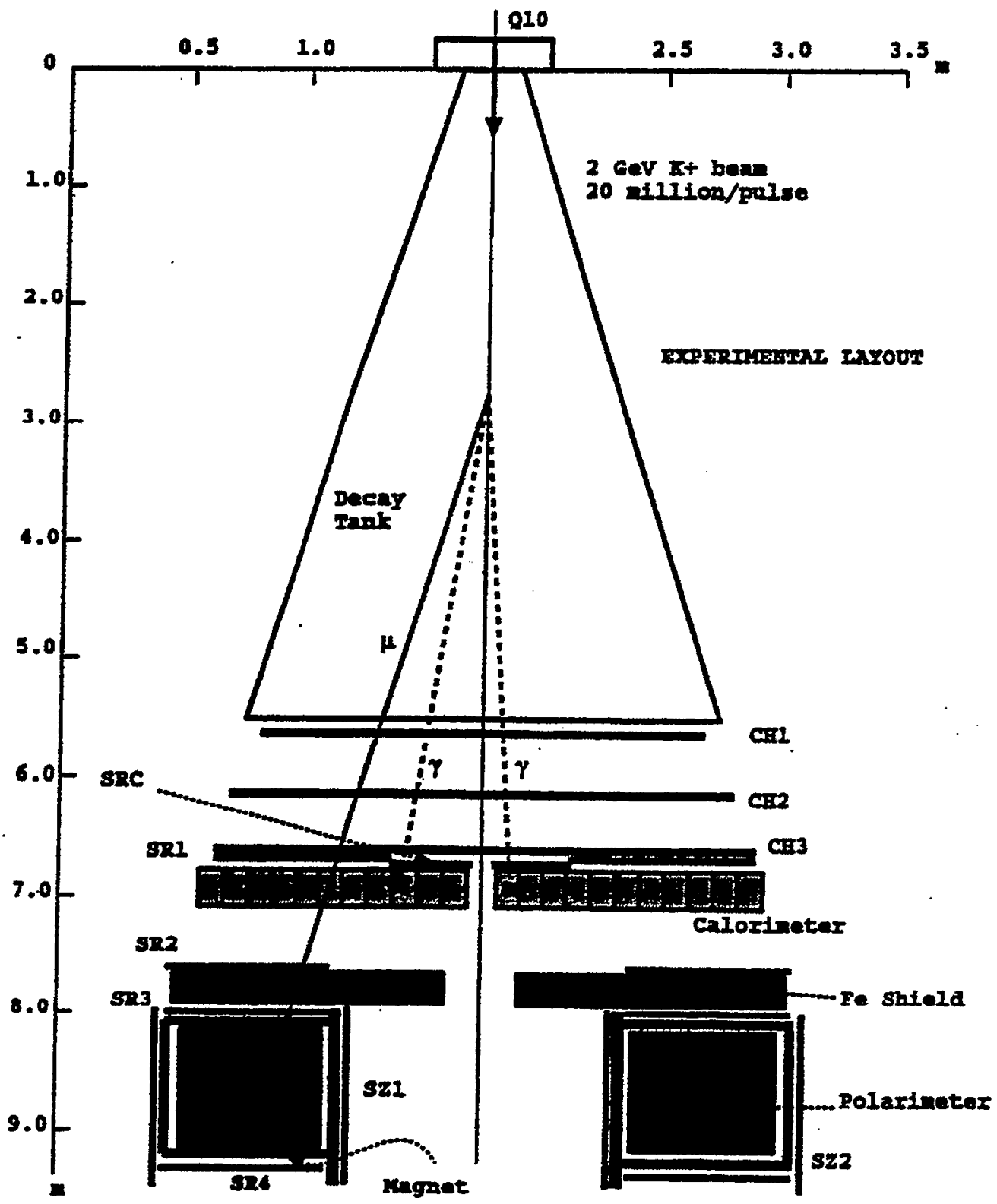

Schematic of the experiment. A typical $K^{+} \rightarrow \mu^{+} \pi^{0} v$ event is superimposed. 


\section{EXPERTMENT 923 - SEARCH FOR T VIOLATING MUON POLARIZATION IN $K^{+} \rightarrow \mu^{+} \pi^{\theta} v_{\mu} D E C A Y$}

SPOKESMEN: M. V. DIWAN, HONG MA, V. ISSAKOV

- Brookhaven National Laboratory - A. S. Carroll, M. V. Diwan, J. Frank, A. Gordeev, S. Kettell, L. Leipuner, L. Littenberg, H. Ma, V. Polychronakas

- Institute for Nuclear Research, Moscow, Russia - G. Atoyan, Y. Andreev, V. Issakov, O. Karavichev, T. Karavisheva, A. Poblaguev, A. Proskuryakov

- Institute for High Energy Physics, Protvino, Russia - V. Semenov

- Louisiana Tech University - M. Elaasar, D. Greenwood, K. Johnston

- Yale University - R. Adair, R. Larsen

This experiment proposed a new search for the time reversal violating polarization of the muon normal to the decay plan of the $\mathrm{K}^{+} \rightarrow \mu^{+} \pi^{\circ} v_{\mu}$ decay. The experiment will be performed with in-flight decays in an intense ( $2 \mathrm{x}$ $10^{7} \mathrm{~K}^{+}$per sec) $2 \mathrm{GeV} / \mathrm{c}$ separated kaon beam in an existing beam line at the AGS. The center piece of the detector will be a new polarimeter which will consist of 128 carbon wedges, with active detector elements (either scintillator or wire chambers) between the wedges, arranged in a cylindrical manner around the kaon beam. More than $10^{9}$ events are expected to be analyzed to obtain sensitivity to the T-violating polarization of \pm 0.0007 to $\operatorname{Im} \xi$, an improvement by approximately 40 over the previous best limit. 


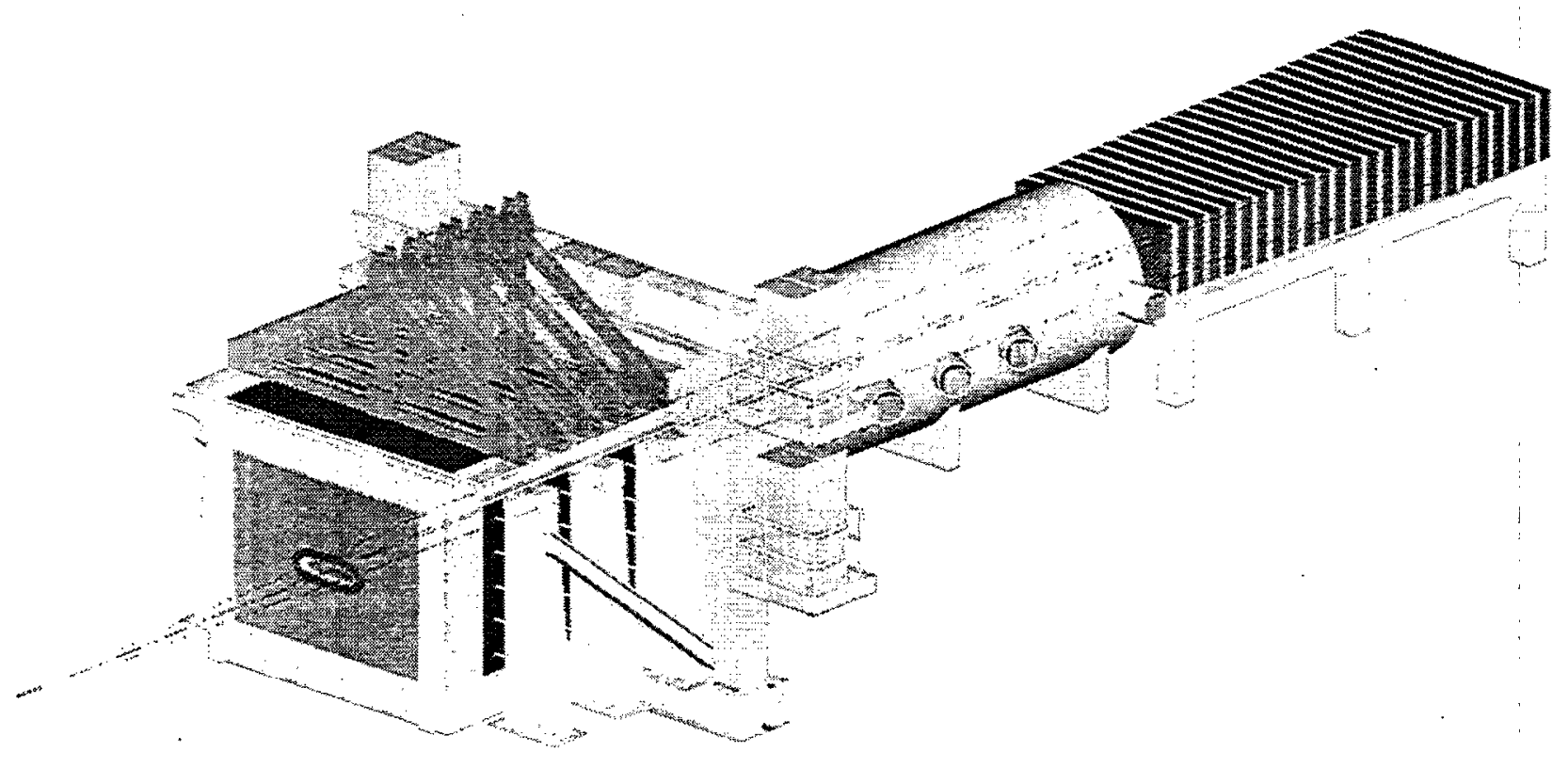

Layout of the $K_{\mathfrak{L}}^{o} \rightarrow \pi^{o} v \bar{v}$ experiment 


\section{EXPERIMENT 926 - MEASUREMENT OF $K_{L}^{0} \rightarrow \pi^{\circ} v \bar{v}($ KOPIO) \\ SPOKESMEN: D. BRYMAN, L. LITTENBERG, M. ZELLER}

- Brookhaven National Laboratory - I-H. Chiang, J. W. Glenn, V. Jain, D. Lazarus, K. Li, L. Littenberg,

G. Redlinger, R. Strand, C. Woody

- INR-Moscow - G. Atoyan, M. Grigoriev, V. Issakov, A. Ivashkin, M. Khabibullin, A. Khotjanzev,

Y. Kudenko, O. Mineev, A. Poblauev

- Kyoto University - T. Nomura, N. Sasao

- Thomas Jefferson National Accelerator Facility - M. Ito

- TRIUMF - E. Blackmore, D. Bryman, P. Gumplinger, M. Hasinoff, A. Konaka, J. Macdonald, T. Numao, R. Poutissou, G. Smith

- Virginia Polytechnic Institute - M. Blecher, M. Pitt, B. Vogelaar

- . Yale University - S. Dhawan, H. Kaspar, S. Pislak, M. Zeller

- University of British Columbia - M. Hasinoff

- University Cincinnati - K.Kineshita

- University of New Mexico - B. Bassalleck, N. Bruner, D. E. Fields, J. Lowe, T. L. Thomas

- University Virginia - D. Pocanic

- University Zurich - P. Tru

This experiment will be a measurement of the branching ratio for the rare decay $\mathrm{K}_{\mathrm{I}} \rightarrow \pi^{0} v \bar{v}$. The method employs kaon time-of-flight and full kinematic reconstruction of the $\pi^{\circ}$ to suppress backgrounds to a level well below an anticipated signal in the range of $3 \pm 2 \times 10^{-11}$. 


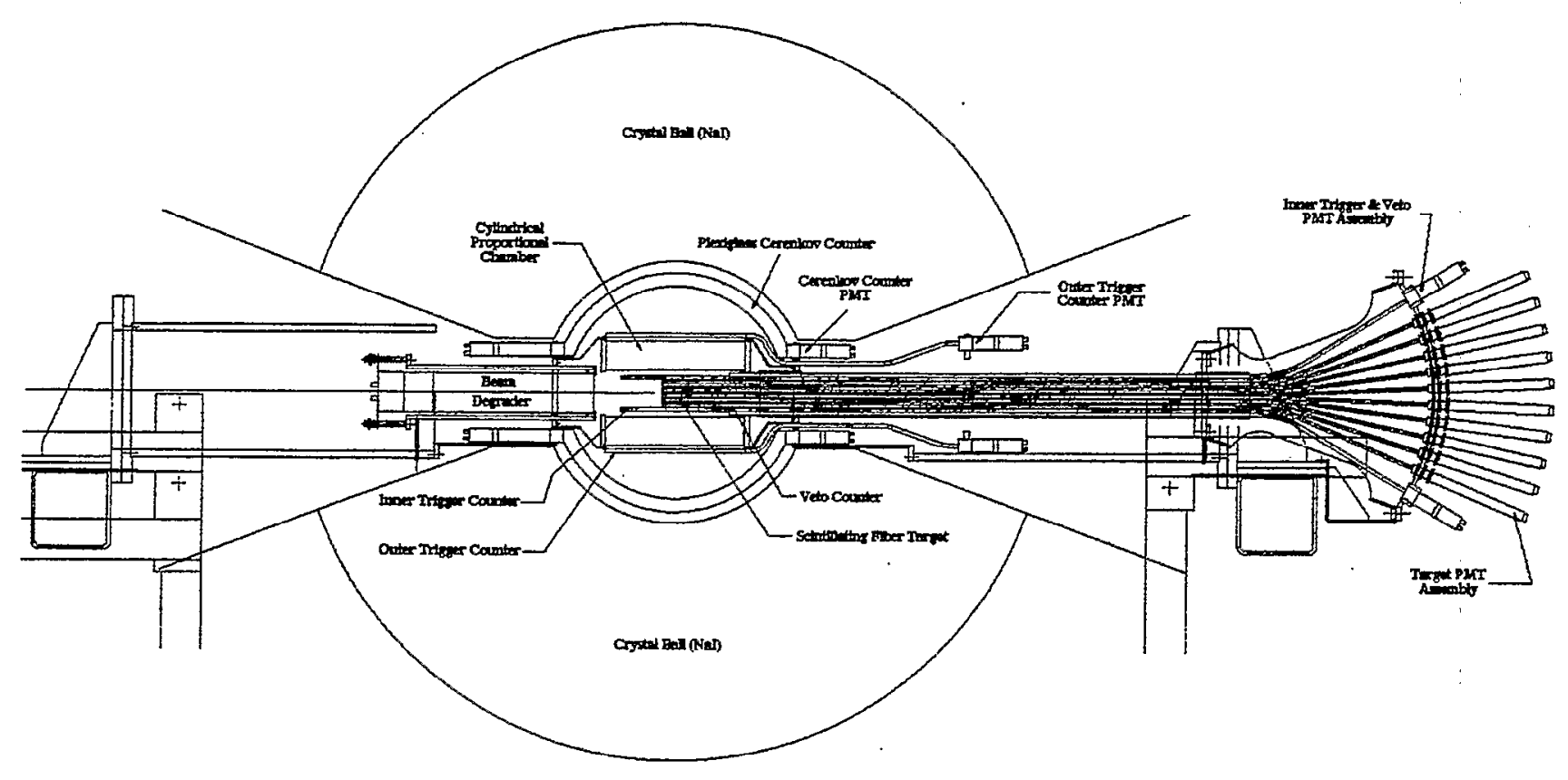




\section{Experiment 927-Measurement of the $K_{e 3}{ }^{+}$Decay Rate and Spectrum}

\section{SPOKESMAN: B. M. K. NEFKENS}

- University of California at Los Angeles - M. Clajus, S. C. McDonald, A. Maru[i], A. M. K. Nefkens, W. B. Tippens

- Joint Inst. for Nuclear Research - Dubna - A. Efendiev

- Abilene Christian University - L. D. Isenhower, M. E. Sadler

- Argonne National Laboratory - H. M. Spinka

- Arizona State University - J. R. Comfort, K. Craig

- Rudjr Bošković Inst., Zagreb, Croatia - M. Batinić, I. Šlaus, I. Supek, A. Švarc

- University of Colorado - R. J. Peterson

- George Washington University - C. Bennhold, W. J. Briscoe

- University of Karlsruhe, Germany - H. M. Staudenmaier

- Kent State University - D. M. Manley

- St. Petersburg Nuclear Physics Inst., Gatchina - A. B. Starostin

- University of Regina, Canada - G. J. Lolos, Z. Papandreou

- Valparaiso University - J. Alyea, D. Grosnick, D. D. Koetke, R.W. Manweiler, S.Stanislaus

The goal of this experiment was to measure the $\mathrm{K}_{\mathrm{e} 3}{ }^{+}$decay rate to better than $0.7 \%$. This translates into an absolute determination of $V_{u s}$ to better than $0.35 \%$, not including the error in the theoretical evaluation of the small correction for the finite quark masses. Combined with the relatively well known values for $V_{u d}$ and $V_{u b}$, this provided the most stringent test of the unitarity of the CKM matrix. Among several reasons that such a test is of interest is the proposed existence of supersymmetry (SUSY) particles which are predicted to cause a small deviation from the unitarity relation. 


\section{E930 setup}

AGS-D6

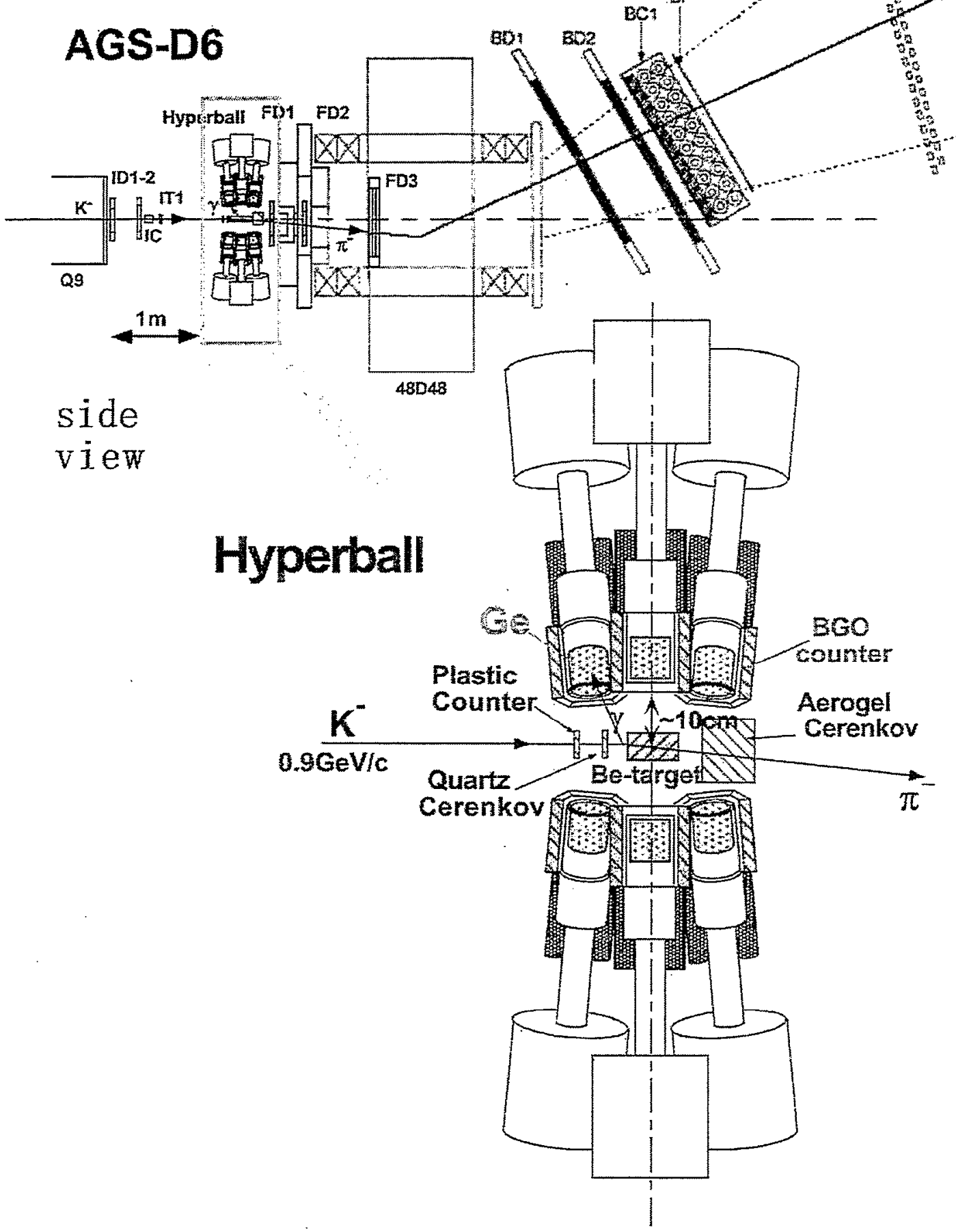




\section{EXPERMMENT 930 - HIGH-RESOLUTION $\gamma$ SPECTROSCOPY OF HYPERNUCLEI USING LARGE-ACCEPTANCE GERMANIUM DETECTOR}

\section{SPOKESMAN: H. TAMURA}

- Brookhaven National Laboratory - D.E. Alburger, R.E. Chrien, H. Hotchi, M. May, P. Pile, A. Rusek, R. Sutter

- Carnegie Mellon University - P. Eugenio, G.B. Franklin, P. Khaustov, B.P. Quinn, R.A. Schumacher

- China Institute of Atomic Energy - S.H. Zhou

- Hiampton University - L. Gran, L. Tang, L. Yuan, X.F. Zhu

- Kyoto University - H. Akikawa, K. Imai

- North Carolina A\&T University - R. Sawafta

- Osaka University - S. Ajimura

- Tohoku University - Y. Miura, J.Sasao, H. Tamura, M. Ukai

- University Freiburg - J. Franz, H.Schmitt

- University of Tokyo - K. Tanida

The experimenters propose a high-resolution $\gamma$-ray spectroscopy measurement of several light $\Lambda$ hypernuclei employing Hyperball, a large-acceptance germanium detector array for hypernuclear studies constructed in Japan. $\Lambda$ hypernuclei are produced by the $\left(\mathrm{K}^{-}, \pi^{-}\right)$reaction and their $\gamma$ transitions are detected in coincidence. They will use $\mathrm{K}^{-}$beam at D6 beam line together with the E813 spectrometer. The aim is to determine all the terms of $\Lambda \mathrm{N}$ spin-dependent interactions, namely, spin-spin, spin-orbit, and tensor forces between $\Lambda$ and nucleon, through detailed level structures of ${ }_{\wedge}^{9} \mathrm{Be},{ }_{\wedge}^{16} \mathrm{O},{ }_{\wedge}^{7} \mathrm{Li}$, and ${ }_{\wedge}^{12} \mathrm{C}$ hypernuclei.

Date for ${ }^{9} \mathrm{Be}$ target were taken in 1998 and the two transitions in ${ }_{n}^{9} \mathrm{Be}, \mathrm{E} 2\left(5 / 2^{+} \rightarrow 1 / 2^{+}\right)$and $\mathrm{E} 2\left(3 / 2^{+} \rightarrow 1 / 2^{+}\right)$, were separately observed at $3.05 \mathrm{MeV}$. Their energy difference was measured t be only $31 \pm 2 \mathrm{keV}$, which confirmed a very small but finite size of the sin-orbit force $\left(\propto 1_{\Lambda N} S_{\Lambda}\right)$ in the $\Lambda N$ interaction. 

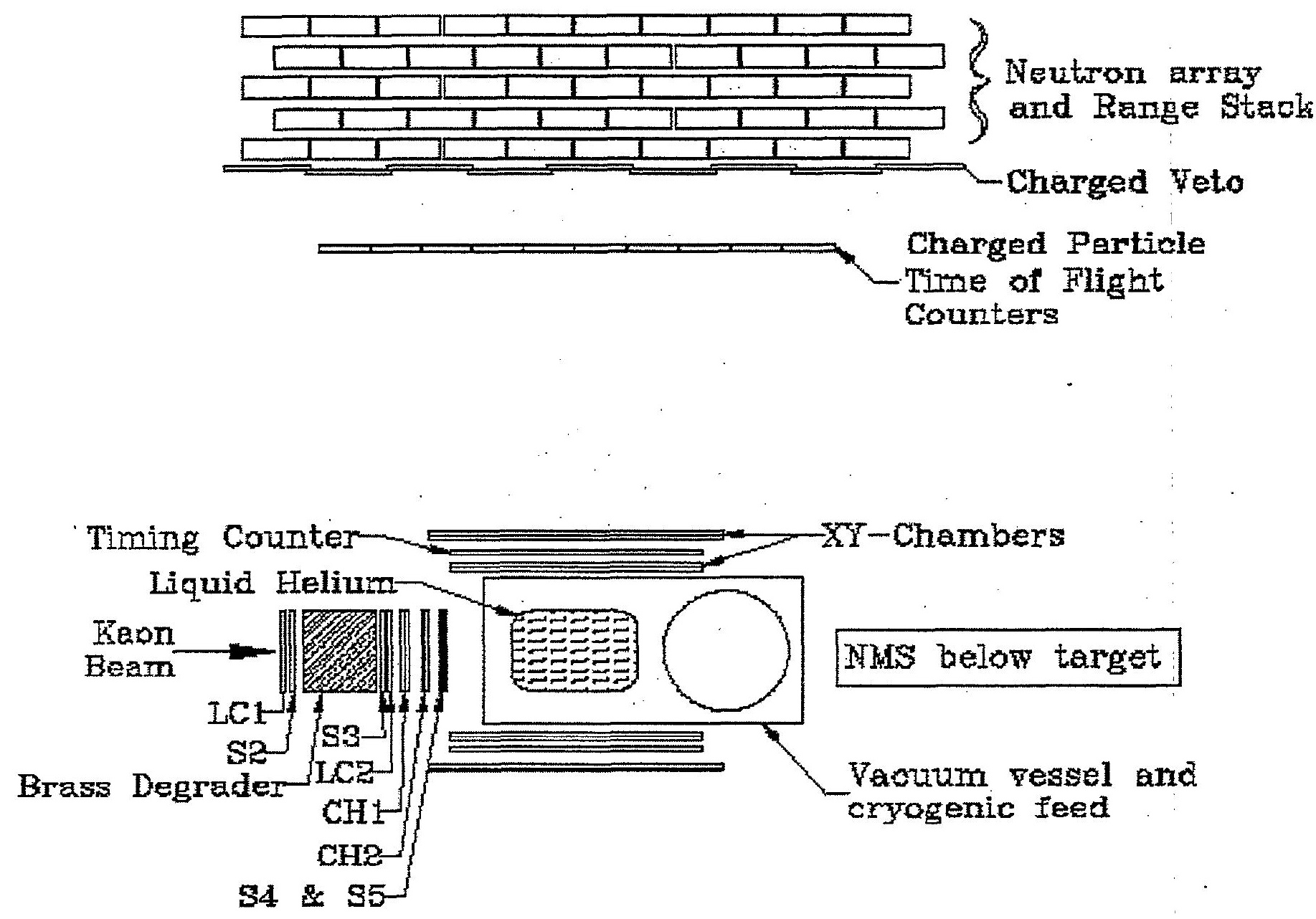

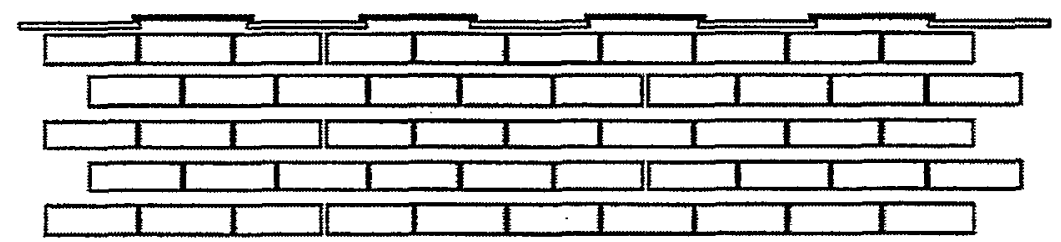




\section{EXPERIMENT 931 - STUDY OF THE $\triangle I=1 / 2$ RULE IN THE WEAK DECAY OF S-SHELL HYPERNUCLET}

SPOKESMAN: D. DEHNHARD, E. HUNGERFORD, V. ZEPS

- Arizona State University - J. R. Comfort, C. Gauland

- Brookhaven National Laboratory - R E. Chrien, M. May, P. H. Pile, A. Rusek, R. Sutter

- Carnegie-Mellon University - G. B. Franklin, B. Quinn

- CIEBAF - L. Tang

- Christopher Newport College - J. Gerald

- George Washington University - W. Briscoe

- Los Allamos National Laboratory - J. Amann, D. Boudrie, C. Edwards, B. F. Gibson. C. Morris, J. O’Donnell. J-C. Peng, A. Thiessen

- Louisiana Tech University - M Barakat. K. Johnston

- North Carolina A\&T - R. Sawafta

- R. Boskovic Institute - I. Supek

- Tohoku University - O. Hashimoto

- University of California at Los Angeles - B. Nefkens, W. B. Tippens

- University of Colorado - G. A. Peterson

- University of Houston - M. Ahmed, X. Cui, A. Empl, E. V. Hungerford, A. Lan, B. Mayes, L. Pinsky

- University of Kentucky - V. Zeps

- University of Maryland - P. G. Roos

- University of Minnesota - D. Dehnhard

- University of Texas at Austin - G. Glass, C. Fred Moore, H. Ward

- University of Zagreb - D. Androic, M. Furic, T. Petkovic, M. Planinic

This experiment addresses an unresolved, fundamental question of "why" and "when" to apply the $\Delta \mathrm{I}=1 / 2$ rule to the weak decay of strange hadrons. An opportunity now exists to determine if this apparently universal rule applies to the non-mesonic weak decay of a $\Lambda$, by studying particle emission from the weak decay of ${ }_{\Lambda}^{4} \mathrm{He}$. The experiment will use the NMS spectrometer and the LESBII beam line. 


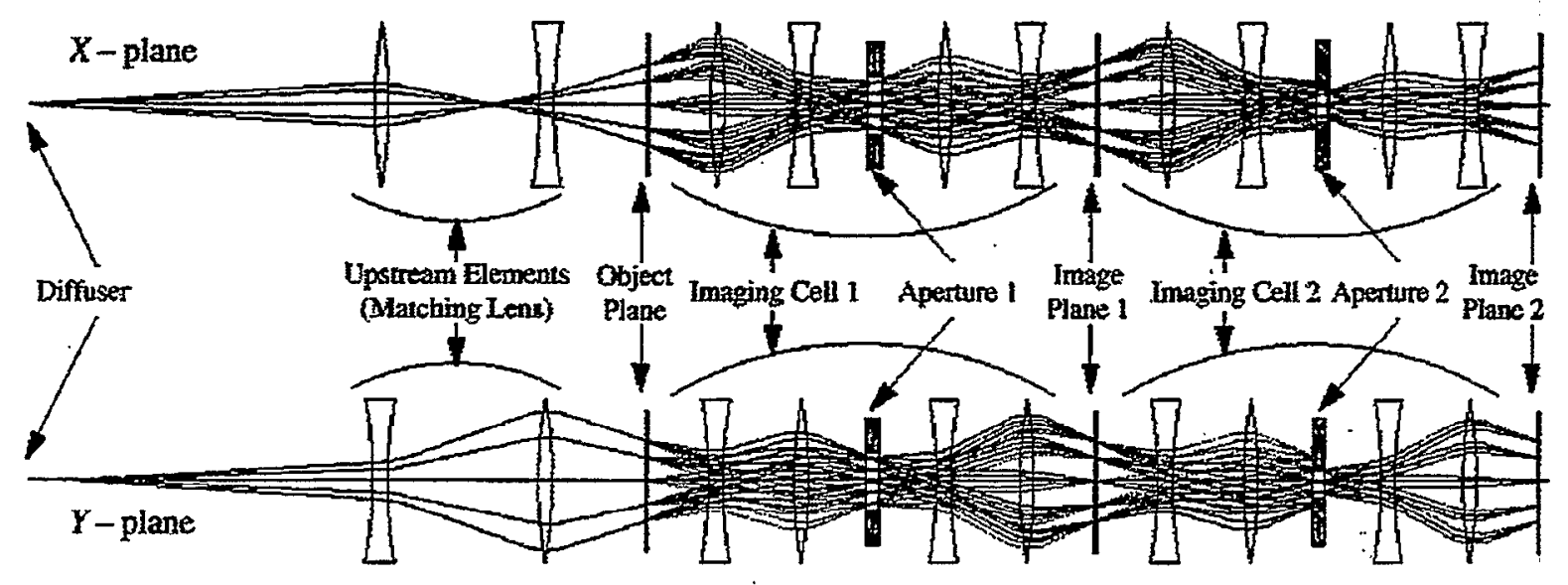

\section{E933 Schematic}

Schematic of the Proton Radiography magnetic lens system showing both the $X$ and $Y$ views. The beam is first prepared with a diffuser and matching lens to meet optics requirements. It then passes through the object being radiographed. The transmitted beam passes through an iris, or aperture located in the middle of the 4-quadrupole -I magnetic lens cell and is focused on the first detector. It then enters the second identical -I lens cell, which this time has a smaller diameter iris, and is focused on a second detector. Together, the two detectors provide the information needed to reconstruct both the density profile and material composition of the object. 


\section{EXPERIMENT 933 - PROTON RADIOGRAPHY}

CONTACT PERSONS: C. MORRIS, E. HARTUNI

- Bechtal Nevada - R. Thompson, R. Liljestrand

- Lawrence Livermore National Laboratory - E. Ables, M. B. Aufderheide, R. M. Bionta,

P. D. Barnes Jr., T. E.Cowan, D. Fujino, E. P. Hartouni, H-S. Park, R. A. Soltz, K. A.VanBibber, D. M. Wright

- Los Alamos National Laboratory - J. F. Amann, R. L. Boudrie, G. E. Hogan, N. S. P. King, A. R. Mathews, J. B. McClelland, K. B. Morley, C. L. Morris, C.T. Mottershead, K. H.Mueller, J. S. Sarracino, G. J. Yates, H. J. Ziock, J. D. Zumbro

Proton radiography was investigated as a promising new technology for hydrodynamic testing in support of a science based stockpile stewardship program. It gave the potential to outperform traditional X-ray Techniques in meeting the full range of requirements that are being developed by a DOE-DP charted tri-lab working group. This group was established to identify technical approaches by which an advanced hydrodynamic testing capability could certify the stockpile in the absence of underground testing. 


\section{BNL-AGS Target Test Experiment}

Schematic section along beam center line

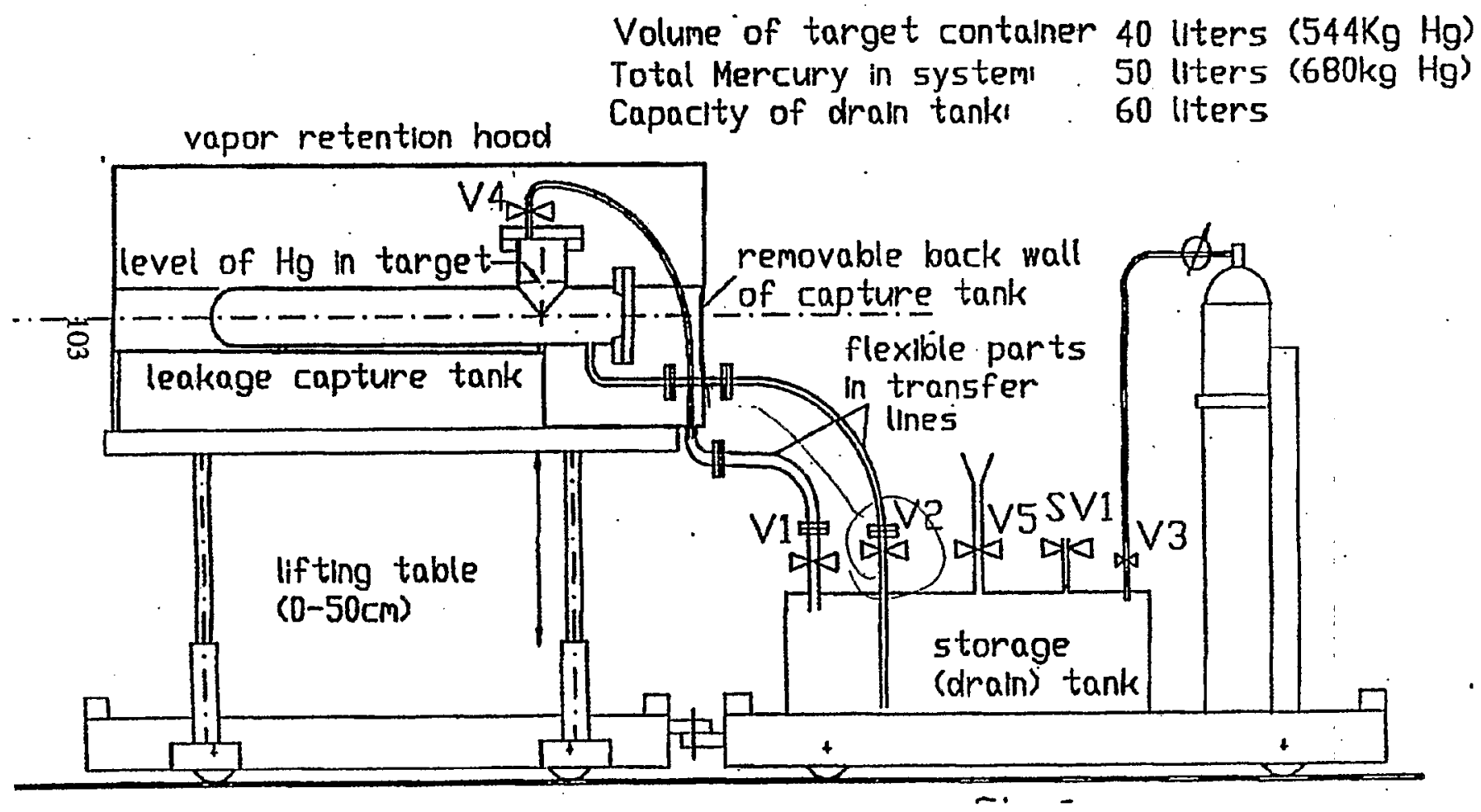




\section{EXPERIMENT 938 - SPALLATION NEUTRON TARGET STUDIES AT THE AGS}

SPOKESMEN: G. BAUER, J. HAINES, J. B. HASTINGS, N. WATANABE

- Argonne National Laboratory - J. M. Carpenter

- Brookhaven National Laboratory - J. B. Hastings

- Forschungszentrum Jülich - H. Conrad

- High Energy Accelerator Research Organization (KEK) - H. Ikeda

- Japan Atomic Energy Research Institute - N. Watanabe

- Los Alamos National Laboratory - G. Russell

- Oak Ridge National Laboratory - J. Haines

- Paul Scherrer Institute - G. Bauer

There is a worldwide effort in planning and designing the next generation neutron source; the focus is on spallation source with average power of up to $5 \mathrm{MW}$ and single pulse energies of 100 kjoules. The design issues center around the heavy metal target; liquid mercury is the current first choice. The AGS is a unique resource to study in detail neutron production, pressure wave mitigation and other issues in target design. It is the only proton source in the world with the energy per pulse equal to the proposed future sources.

This experiment measured the neutron production and energy deposition in liquid mercury as a function of incident proton energy. The pressure wave behavior under peak power loading was measured and mitigation measures evaluated. Finally, the performance of a liquid mercury target in conjunction with a lead reflector and moderators were measured to study the complete system performance. The total proton fluence required at a given proton energy was minimal (typically a few time $10^{14}$ ) for the first two measurement periods. 


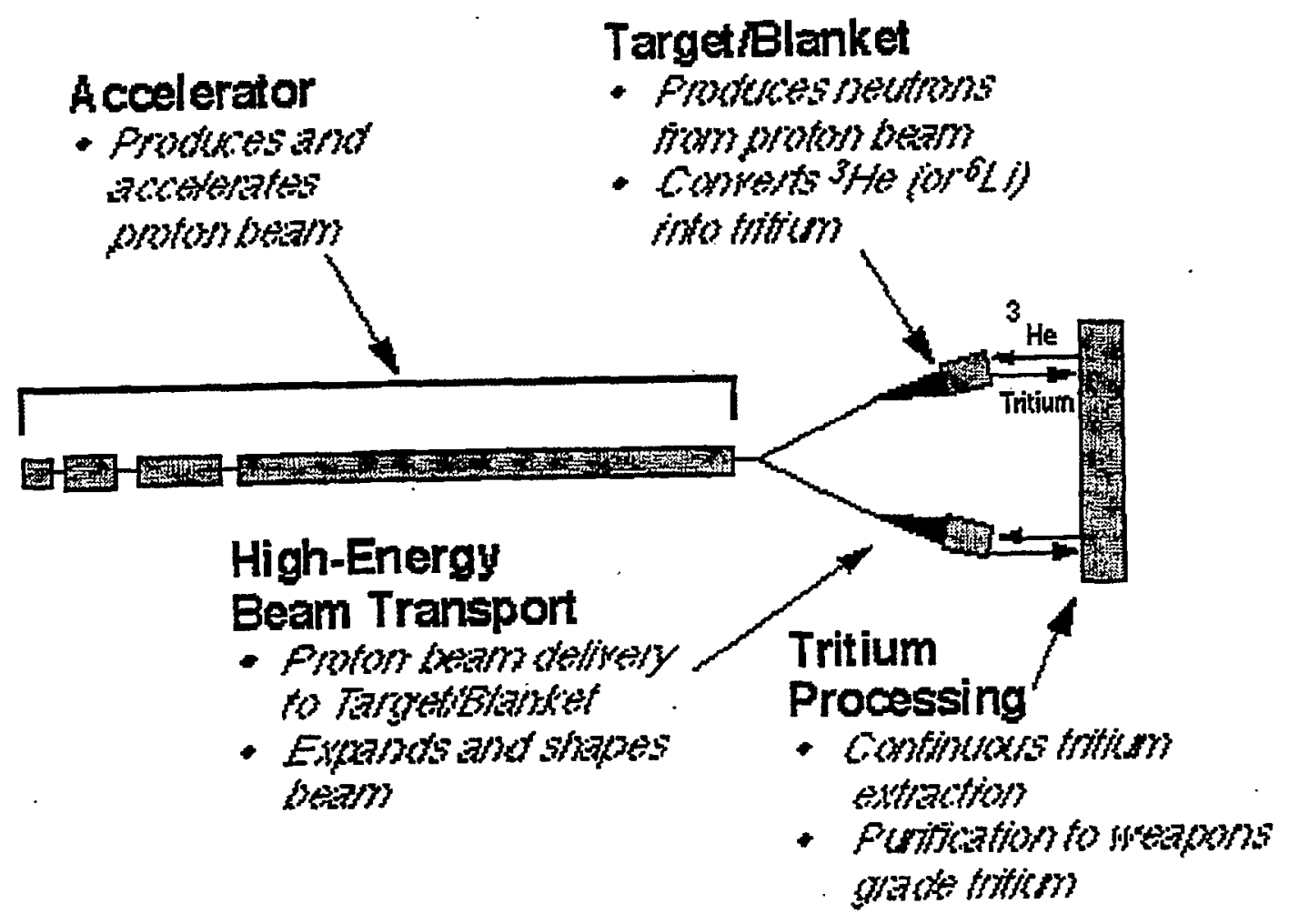


EXPERIMENT 939 - EXPERMENTS FOR THE DOE ACCELERATOR PRODUCTION OF TRITTUM (APT) PROJECT

SPOKESMEN: N. S. P. KING AND A. L. HANSON

- Bechtel Nevada - R. Thompson, R. Liljestrand

- Brookhaven National Laboratory - G. Greene, A. L. Hanson, L. Snead, M.Todosow

- CEA - J. Frehaut

- Lawrence Livermore National Laboratory - G. Henry, D. Slaughter

- LNS-CNRS - S. Leray

- Los Alamos National Laboratory

K. Alrick, F. Cverna, N. S. P. King, G. L. Morgan, L. Waters, J. Ullmann

- U. S. Department of Energy - T. Ward

The purpose of this experiment was to advance the $R \& D$ work for the accelerator production of tritium as a part of a national program now underway in this subject. Using fast extracted protons, thin aluminum foils were exposed to proton beams of $800,1200,1600$ and $2000 \mathrm{MeV}$ to measure the production cross sections for the ${ }^{27} \mathrm{Al}(\mathrm{p})^{24} \mathrm{Na}$ and ${ }^{27} \mathrm{Al}(\mathrm{p})^{22} \mathrm{Na}$ reations. These cross-sections were used to help normalize data from other measurements. 


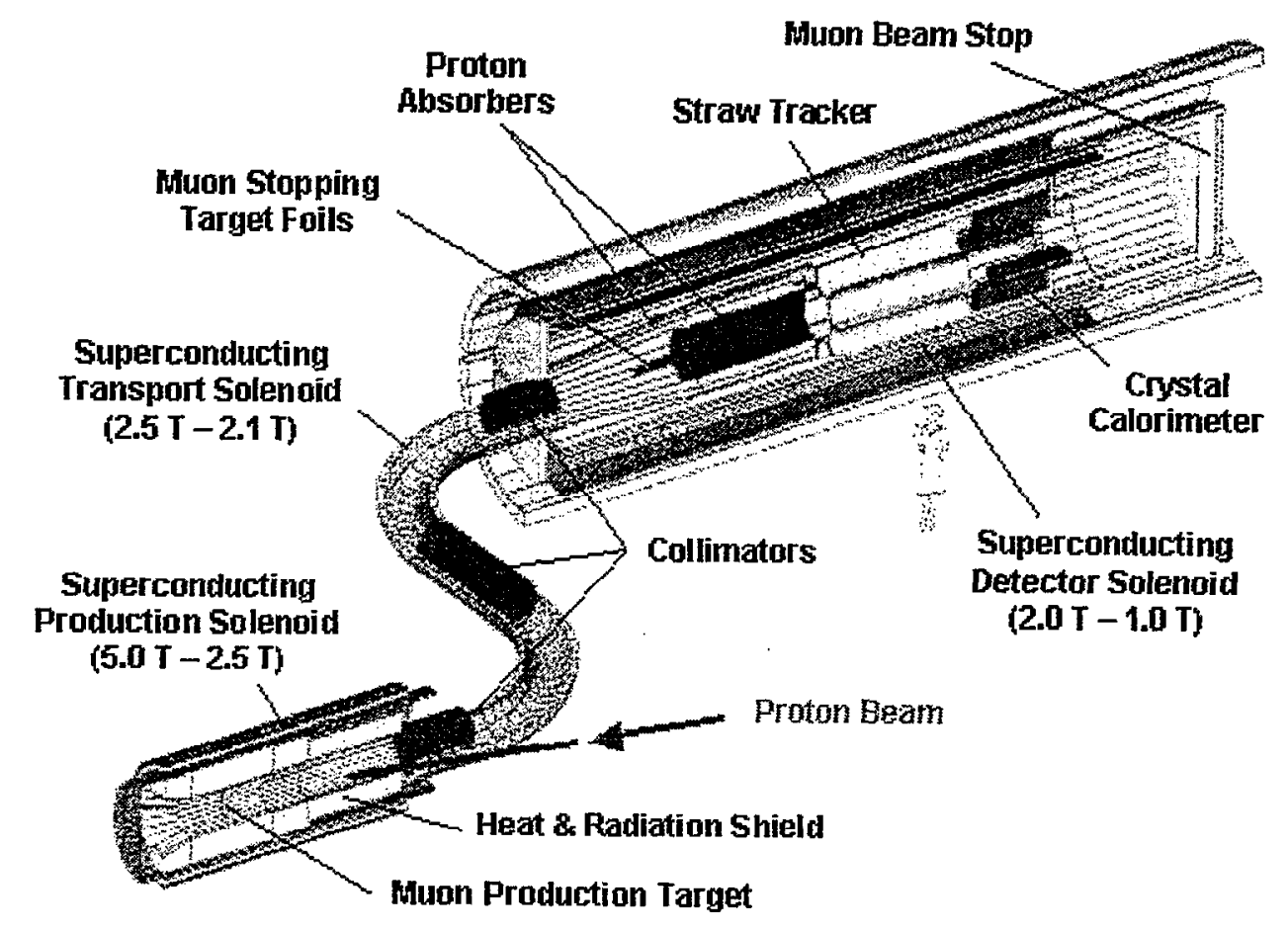




\section{EXPERIMENT 940 - A SEARCH FOR $\mu^{-} N \rightarrow e^{-} N$ WITH SENSITIVITY BELOW $10^{-16}$ MUON-ELECTRON CONVERSION (MECO)}

SPOKESMAN: W.MOLZON

- Institute for Nuclear Research - R. M. Djikibaev, V. M. Lobashev, A. N. Toropin

- New York University - A. Mincer, P. Nemethy, J. Sculli

- Purdue Univeristy - S. Carabello, D. Koltick

- University of California at Irvine - M. Bachman, G. Kagel, R. Lee, T. J. Liu, W. Molzon, M. Overlin

- University of Houston - A. Empl, E. V. Hungerford, K. J. Lan, B. W. Mayes, L. S. Pinsky, J Wilson, M. Youn

- University of Pennsylvania - W. D. Wales

This experiment proposes to search for the process $\mu^{-} \mathrm{N} \rightarrow \mathrm{e}^{-} \mathrm{N}$ with a significantly improved sensitivity with respect to past and proposed future searches. Interest is in searching for violations of additive quantum numbers associated with each type of lepton. Violation of these quantum numbers is commonly referred to as lepton flavor violation (LFV). The process will provide direct evidence of muon and electron number violation.

The experiment will be conducted in a new $\mu$ beam line produced using a pulsed proton beam. The proton energy will be chosen in the range $8-20 \mathrm{GeV}$ to optimize the $\mu$ flux per unit time and minimize operating costs of the experiment. The expected sensitivity, normalized to the kinematically similar process of $\mu$ capture on the nucleus, is one event for a branching fraction of $2 \times 10^{-17}$. 


\section{EXPERIMENT 945 - PHYSICS BENCHMARK MEASUREMENT OF \\ ENERGY DEPOSITTON IN AN APT TARGET/BLANKET PROTOTYPE}

\section{SPOKESMEN: R. COOPER AND G. MORGAN}

- Bechtel Nevada - R. Cooper, J. Langenbrunner, R. Liljestrand, A. Whiteson

- Brookhaven National Laboratory - A. L. Hanson

- General Atomics - M. Madlener, T. Torres

- Lawrence Livermore National Laboratory - D. Slaughter

- Los Alamos National Laboratory - P. Ferlguson, N. King, G.Morgan, E. Pitcher, L. Waters, K. Woloshun

In order to finalize the design of the APT target/blanket, a code validation experiment needed to be performed which measured the energy deposition from a proton beam, as a function of position, in a prototype target/blanket. The measured distribution of the deposited energy was compared with the distribution calculated by the MCMPX transport code. This validation is needed to understand uncertainties in the calculations, which were used to optimize the target/blanket design and maximize the tritium production rate.

This experiment used proton beams with two energies, 1.2 and $1.6 \mathrm{GeV}$, and with the line operating at maximum current, with approximately $5 \times 10^{12}$ protons per spill. 


\section{EXPERIMENT 945A}

Home Page http://www-phys.llnl.gov/N Div/APT/ 


\title{
EXPERIMENT 945A - MEASUREMENT OF RADIATTON DAMAGE INMETALS AT LIQUTD HELIUM TEMPERATURE BY RESISTTVTTY CHANGE
}

\author{
SPOKESMEN: G.A.GREENAND W.F. SOMMER
}

- Brookhaven National Laboratory - B.S. Bowerman, C. C. Finfrock, G.A. Greene, A. L. Hanson, C. L. Snead

- Los Alamos National Laboratory - M. R. James, W. F. Sommer, L. S. Waters

An experiment for code validation purposes is needed to measure resistivity changes in several pure metals after defects have been introduced at liquid-helium temperature by exposure to energetic protons. This data is needed to quantify the uncertainties in the calculations used to predict radiation damage to spallation targets under proton irradiation. Wire samples will be tested in a cryostate that were used for similar studies at BNL.

This experiment was installed in the U-line across from the UGE2 entrance, and was directly upsteam from E945, the LANL energy deposition experiment. Experiments were conducted at four proton energies: $0.8,1.2$, 1.6 and $2.0 \mathrm{GeV}$, and with the beam line operating at maxim current, with approximately $5 \times 10^{12}$ proton per spill. It was desirable to have at least two measurements at each energy. A tight beam profile was required for this experiment to minimize heating of the sample holder. The beam was focused to give a spot diameter at the target location of no larger than $1.0 \mathrm{~cm}$ full-width at half-height. 

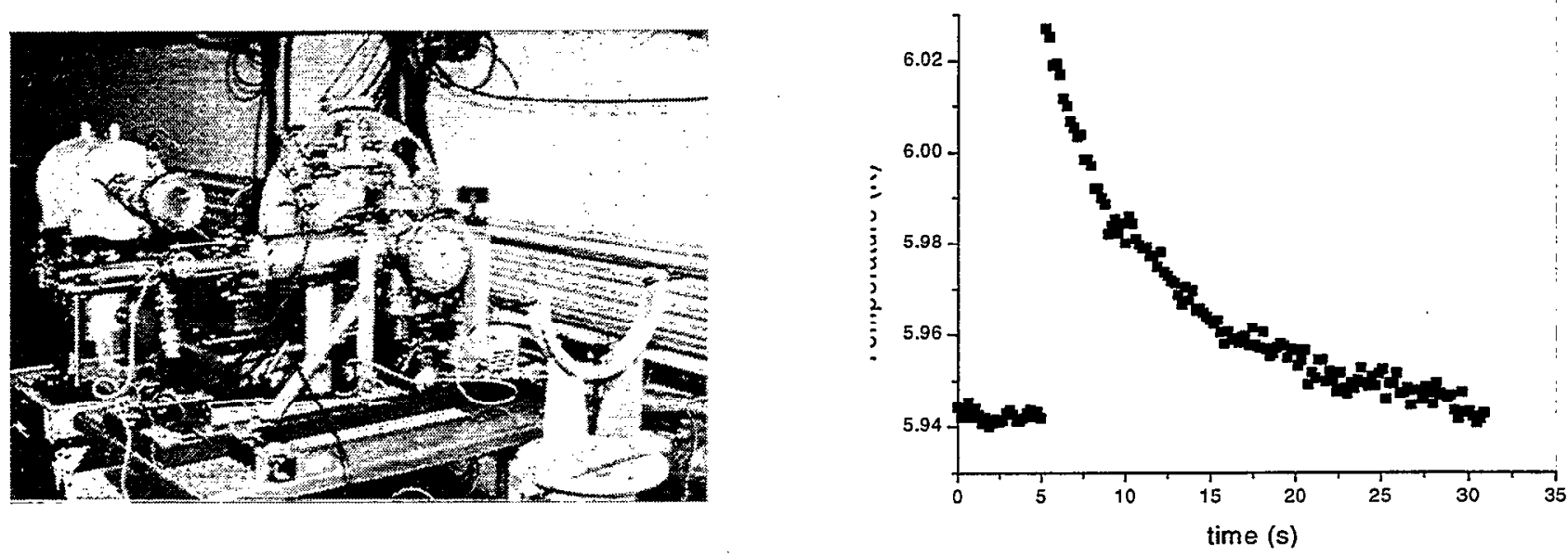

Photograph of the Energy Deposition Vacuum-Cryostat Apparatus in the Beam Line Along With a Typical Thermal Spike of the Instrumented Copper Target 


\section{EXPERTMENT 945B - THERMAL EFFECTS OF SECONDARY RADLATION \\ FROM SPALLATION TARGETS ONMAGNET MATERIALS AT \\ LIQUID HELIUM TEMPERATURE}

SPOKESMAN: G.A.GREEN

- Brookhaven National Laboratory - C. C. Finfrock, G.A. Greene, D. A. Harder

- Los Alamos National Laboratory - P. Walstrom

This experiment was installed in the U-line blockhouse downstream of the UGE2 entrance and directly along side Experiment 933 , off-axis of the primary beam transport. A vacuum cryostat with an instrumented OFHC copper target was pumped down to $10^{-9}$ Torr and cooled to about $6 \mathrm{~K}$ by liquid helium. A series of experiments were conducted with $24 \mathrm{GeV} / \mathrm{c}$ protons in single-pulse extraction mode at an intensity of $5 \times 10^{10}$ protons per bunch onto the E-933 tungsten object. The E-945B copper target was intended to provide a temperature signal in response to the energy deposited by the scattered radiation from the E-933 target, similar to the energy deposition that a superconducting magnet downstream from such a target would experience. After calibration of the copper target by measuring the thernal spikes due to the energy deposition from well characterized electric pulses $(15 \mathrm{mK} / \mathrm{mJ})$, the thermal response of the copper target was measured due to the energy deposition from the scattered charged particles and photons from the spallation target. The temperature instrumentation on the copper target was capable of temperature measurement precision of $10^{-3} \mathrm{~K}$ and accuracy of $3 \times 10^{-3} \mathrm{~K}$, making possible the measurement of energy deposition per unit mass in the copper target as low as 2 $\mathrm{mJ} / \mathrm{kg}$. A photograph of the cryostat assembly is shown, on opposite page, along with an example of a temperature spike $(0.085 \mathrm{~K})$ due to the deposition of $5.6 \mathrm{~mJ}$ into the copper target. 


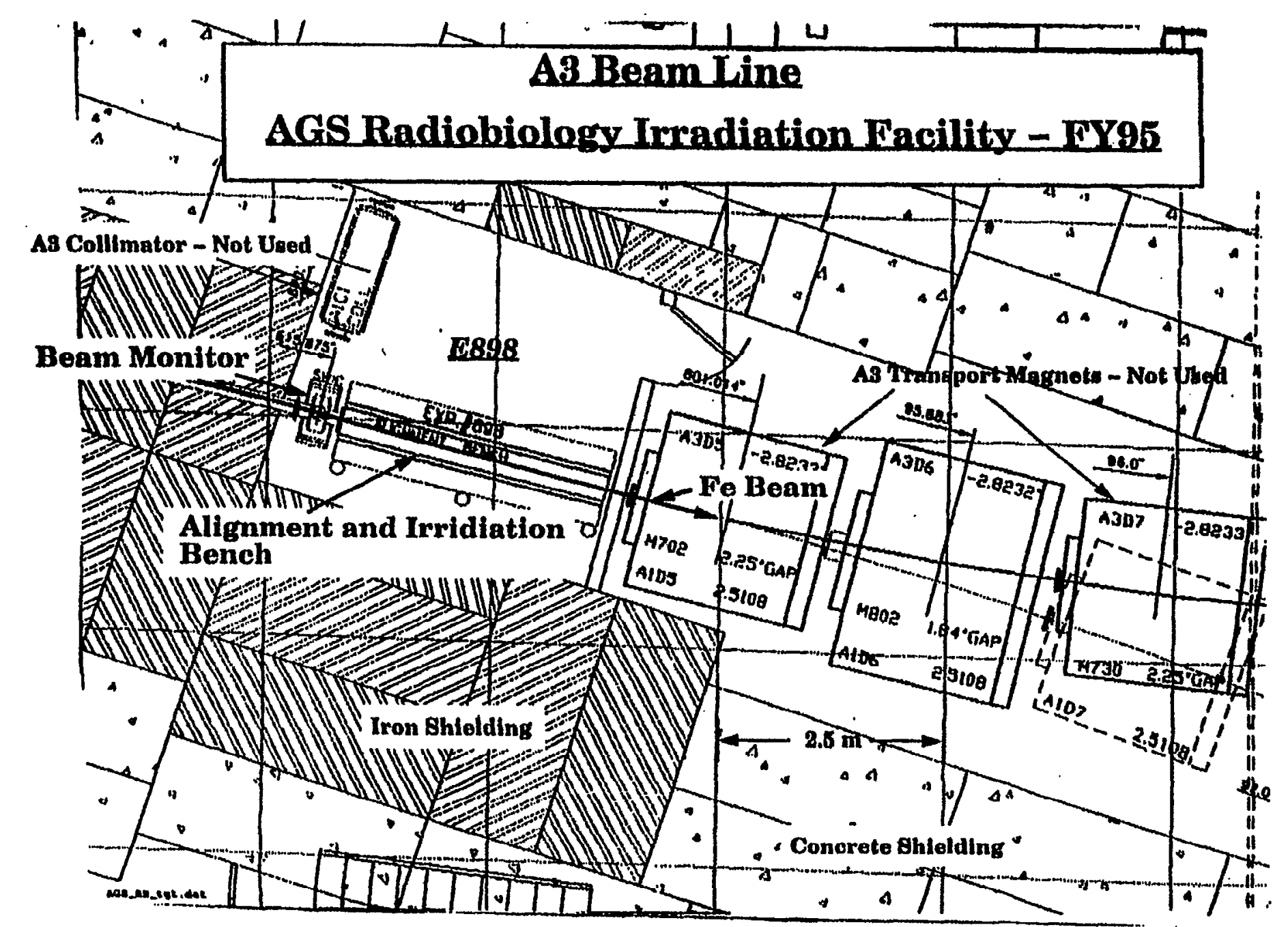




\section{EXPERIMENT 947-NASA - RADIOBIOLOGY TESTING}

\section{SPOKESMEN: M. VAZQUEZ}

- Allabama A\&M University - P. Kale

- Brookhaven National Laboratory - B. Sutherland, M. Vazquez

- Case Western Reserve University - H. Evans

- Colorado University - J. Bedford, C. Waldren

- Columbia University - T. K. Hei

- John Hopkins Medical Inst. - J. Dicello

- Lawrence Berkeley Laboratory - H. Barcellos-Hoff, J.Miller, P. Cooper, A. Kronenberg

- Los Alamos National Laboratory - D. J.Chen

- Pacific Northwest National Laboratory - N. Metting

- Texas A\&M University - R. R. Sinden

- University of Maryland - B.Rabin

- Washington State University - A. L. Brooks

This is a continuing program of experiments in radiobiology, funded by the National Aeronautics and Space Agency (NASA) as part of their space-related research efforts in the life sciences. 
Home Page http://wwww.phy.bnl.gov/e949/

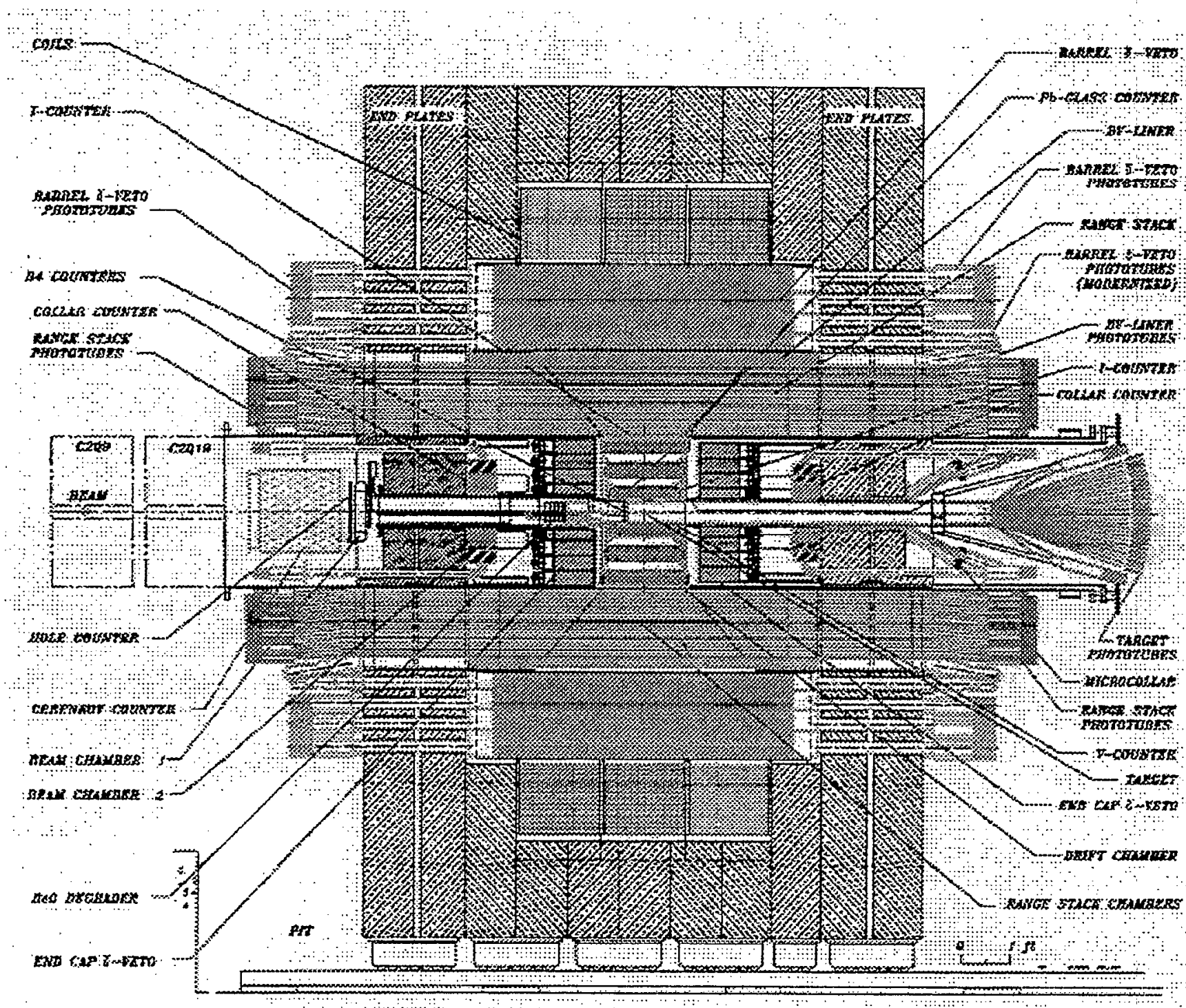

The new detectox. 


\section{EXPERIMENT 949 - ANEXPERIMENT TO MEASURE THE BRANCHING RATTO}

$$
B\left(K^{+} \rightarrow \pi^{+} v \bar{v}\right)
$$

\section{SPOKESMEN: D. BRYMAN, S. KETTELL, S. SUGIMOTO}

- Brookhaven National Laboratory - B. Bhuyan, I-H. Chiang, M. V. Diwan, J.S. Frank, J. S. Haggerty, D.E. Jaffe, S. H. Kettell, K.K. Li, L.S. Littengerg, G. Redlinger, R.C. Strand, B. Viren

- Centre for Subatomic Research-U. Alberta - P. Kitching

- $\quad$ FERMI National Laboratory - P.S. Cooper, E. Ramberg, R.S. Tschirhart

- JFukui University - M. Miyajima, J. Nishide, T. Shimoyama, Y. Tamagawa

- Inst. High Energy Physics - A. Kozjevnikov, L. Landsberg, V. Mukhin, V. Obraztsov, S. Petrenko, V. Rykalin, V. Victorov

- Inst. Nuclear Research - A.P. Ivashkin, M.M. Khabibullin, A.N. Khotjantsev, Y.G. Kudenko, A.S. Levchenko, O.V. Mineev, N.V. Yershov

- KEK - T. Inagaki, S. Kabe, M. Kobayashi, T.K. Komatsubara, K. Omata, T. Sato, S. Sugimoto, T. Tsuneimi, T. Yasuno, Y. Yoshimura, T. Yoshioka

- Kyoto University - T. Fujiwara, T. Nomura

- National Defense Academy of Japan - T. Shinkawa

- Osaka Univrsity - M. Nomachi

- Research Center for Nuclear Physics, Osaka University - T. Nakano, N. Muramatsu

- TRIUMF - P. C. Bergbusch, E. W. Blackmore, S. Chen, J. Hu, A. Konaka, J. A. Macdonald, J. Mildenberger, T. Numao, J-M. Poutissou, R. Poutissou

- University British Columbia - D.A. Bryman, X. Li

- University New Mexico - B. Bassalleck, B. Lewis, J. Lowe

- Yeshiva University - M. Pommot-Maia

A new, more precise measurement of the $\mathrm{K}^{+} \rightarrow \pi^{+} \nu \bar{v}$ branching ratio is proposed in this experiment. Improvements to the E787 apparatus and running mode will be made to reach a sensitivity of (8-14) $\mathrm{x}$ $10^{-12}$, an order of magnitude below the Standard Model prediction. This should result in a determination of $\left|V_{t d}\right|$ to better than $27 \%$. 


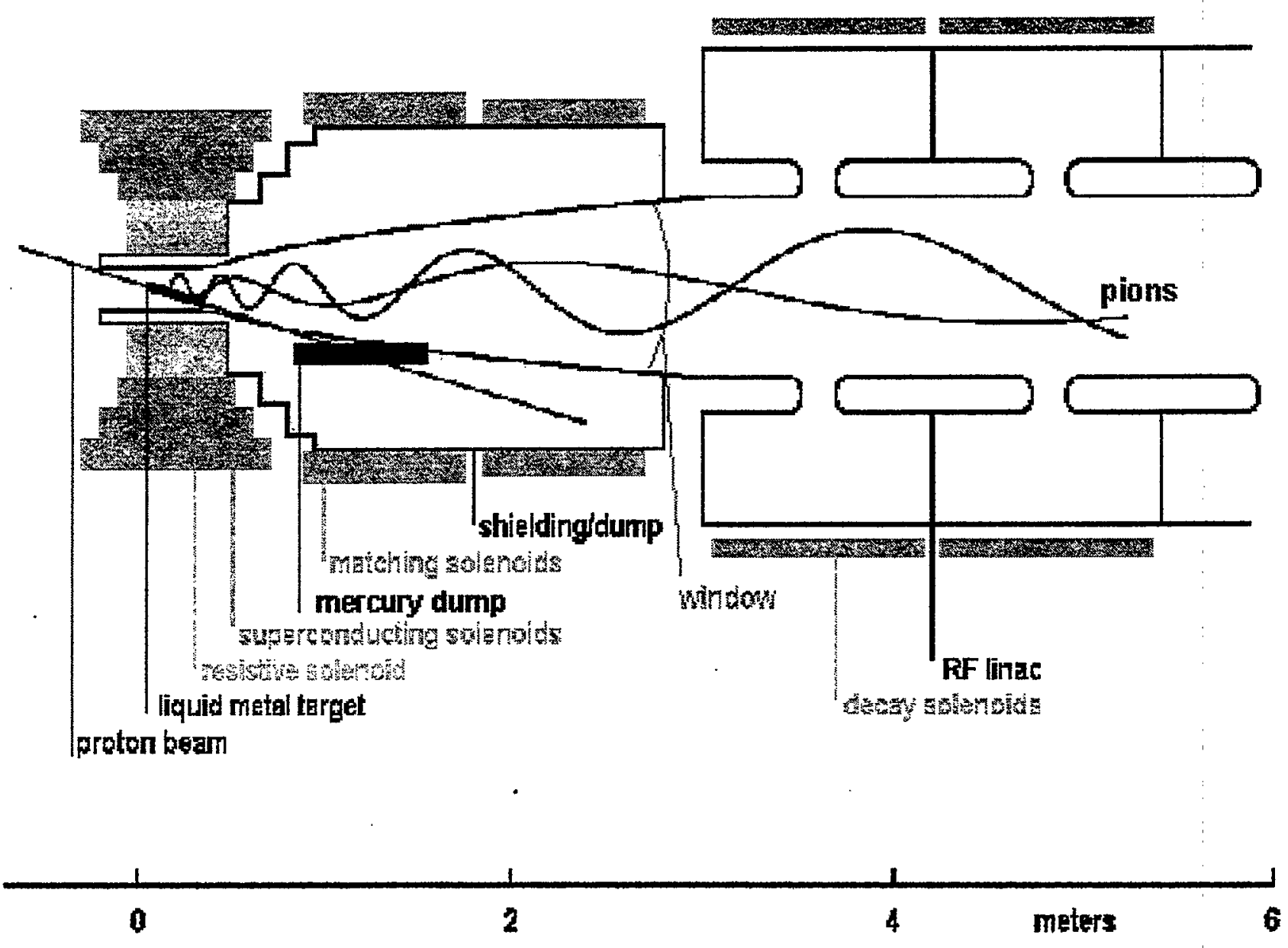

Muon Collider Targetry and Phase Rotation 


\title{
EXPERTMENT 951-ANR\&D PROGRAM FOR TARGETRY AND CAPTURE AT A MUON-COLLIDER SOURCE
}

\author{
SPOKESPERSON: K. T. MCDONALD
}

- Argonne National Laboratory - A. Hassanein, J. H. Norem, C. Reed, D. L. Smith

- Brookhaven National Laboratory - J. Alessi, R. C. Fernow, J.C. Gallardo, J. Hastings, S. A. Kahn, B. J. King, H.G. Kirk, V. LoDestro, R. B. Palmer, T. Roser, Y. Torun, H. Wang, R. Weggel, Y. Zhao

- CERN-C. Johnson,

- Fermi National Laboratory - P. Lebrun, N. V. Mokhov, A. van Ginneken

- Lawrence Berkeley National Laboratory - J. Corlett, Y. Fukui, M. A. Green, R. M. Scanlan,

- Oak Ridge National Laboratory - D. D. Earl, T. A. Gabriel, J. R. Haines,

- Joseph Henry Laboratories, Princeton U. - C. Lu, K. T. McDonald, E. J. Prebys

- SUNY Stony Brook - Y. Torun

The proposed R\&D program plans to investigate targetry issues for a muon-collider source consisting of the initial studies of liquid (and solid) target materials with a proton beam at the AGS. Studies of a liquid-metal jet entering a 20-T magnet at the Natl. high Magnetic Field Lab (NHMFL) in Florida as well as studies of a full-scale liquid-metal jet in a beam of $10^{14}$ protons per pulse, but without magnetic field will be done. It is also planned to study a liquid-metal jet + proton beam $+20-\mathrm{T}$ pulsed solenoid magnet and studies of a $70 \mathrm{MHz}$ rf cavity downstream of the target in the proton beam, but without a magnet around the if cavity. Characterization of the pion yield downstream of the target $+\mathrm{rf}$ cavity and simulation of the performance of liquid-metal targets; thermal shock, eddy currents will be studied. 


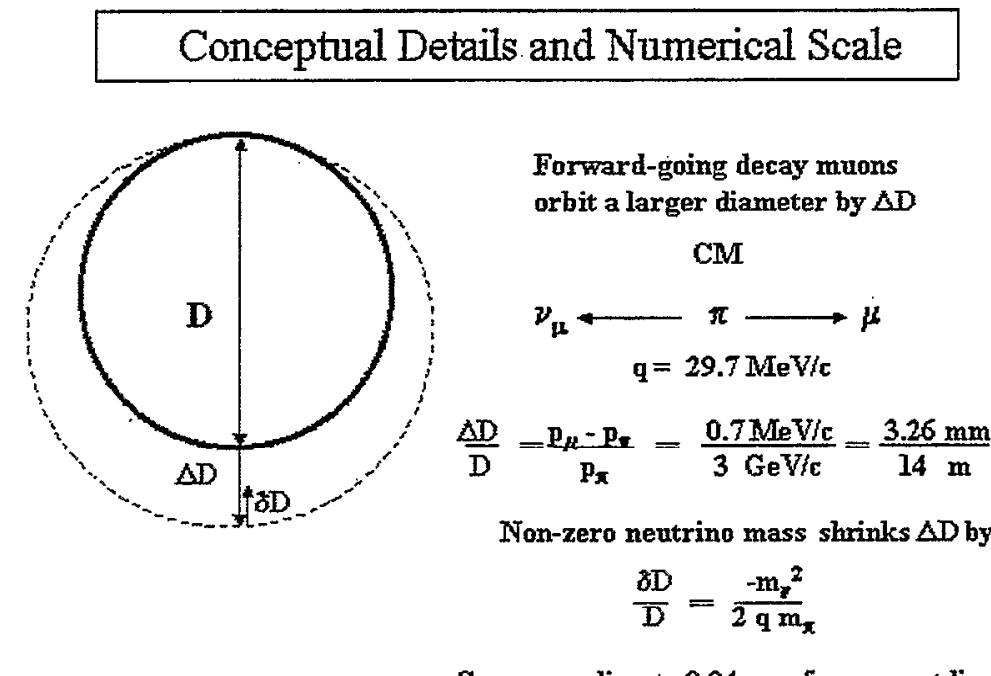

\section{E952: NuMass}

$20 \mathrm{keV} / \mathrm{c}^{2}$ Direct Muon Neutrino Mass Limit

The $14 \mathrm{~m}$ diameter $\mathrm{g}-2$ Storage Ring becomes a one-turn Spectrometer observing $\pi \rightarrow \mu \nu$ decay in flight

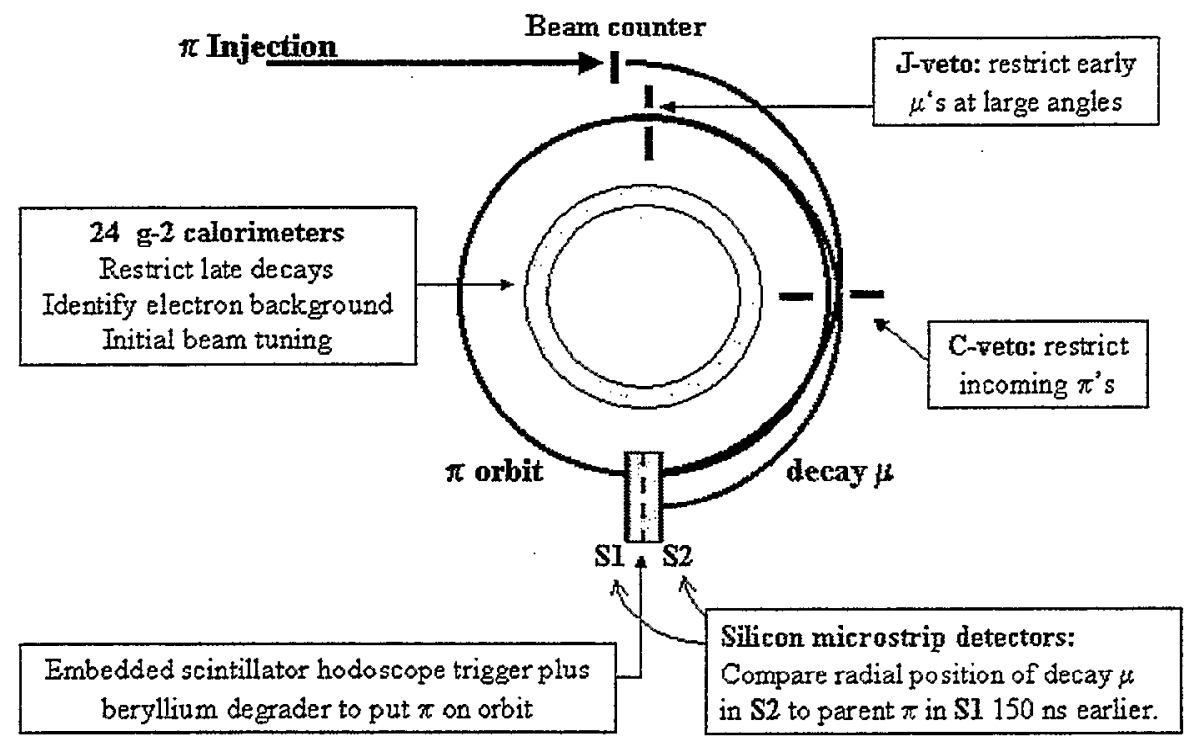




\title{
EXPERIMENT 952-AN IMPROVED LIMIT ON THE MUON NEUTRINO MASS FROM PION DECAY IN FLIGHT
}

\author{
SPOKESPERSON: P. B. CUSHMAN
}

- Boston University - R.M. Carey, J.P. Miller, O. Rind, B.L. Roberts

- Brookhaven National Laboratory - H. Brown, G. Bunce, J.W. Glenn, Y.Y. Lee, W. Meng, W.M. Morse, C. Ozben, R. Prigl, Y.K. Semertzidis

- New York University - P. Nemethy

- Newman Laboratory, Cornell University - Y. Orlov

- Physik Inst. der Universit8 ot Heidelberg - K. Jungmann, G. zu Putlitz

- University Illinois - P.T. Debevec, D.W. Hertzog, C.J.G. Onderwater

- University of Minnesota - P. B. Cushman, A. Heering, I. Kronkvist

- Yale University - F.J.M. Farley, V.W. Hughes

The g-2 Storage Ring is a unique facility for precision measurements that test the standard model. This experiment hopes to improve the muon neutrino mass limit by a factor of 20 (from $170 \mathrm{keV}$ down to $8 \mathrm{keV}$ ) which will be the largest factor improvement made in any neutrino species mass within the last 20 years. It utilizes the storage ring as an extremely uniform spectrometer to compare the parent pion and daughter muon momenta from the $\pi \rightarrow \mu v$ decay in flight. The experiment avoids multiple scattering limitations by transforming the momentum measurement into a position measurement; the edge of the $\mu$ radial distribution referenced on an event-by-event basis to the parent $\pi$ depends on the mass of the recoiling $v$.

In order to put pions on orbit in the ring, $5.2 \mathrm{~cm}$ of beryllium degrades the pion energy. Silicon micro-strip detectors on either side of the degrader record the position of the particle exiting through S1 and then entering the other side through S2 one cycle later. Because of the excellent magnetic field uniformity (better than $1 \mathrm{ppm}$ ), pions which do not decay end up back at the same position on the detector from which they started, no matter what their initial momentum and angle, whereas the daughter muons will cover a range of positions. The resulting radial distribution, referenced on a particle-by-particle basis to the parent pion, will consist of a large narrow peak at zero for undecayed pions and a broad distribution representing the decay products. The fact that the pions return to the origin makes the experiment relatively insensitive to the initial pion distribution. The width of the undecayed pion peak provides an in situ calibration of the orbital parameters and magnetic field uniformity.

Since the muons which decay in the forward direction actually have a higher momentum than the parent pion, they will traverse a larger diameter circle. Muons that are produced in the forward direction at a point halfway around the ring will therefore have the maximum radial displacement from the parent pion initial impact point when they return to the detector. In fact, they represent the maximum displacement of any daughter muon with any decay angle. Thus, the radial distribution of the decay muons referenced on a particle-by-particle basis to the radial position of their parent pions has a well-defined edge. If the neutrino has mass, this will reduce the energy of the forward-going muon and shrink the edge of the decay muon distribution by an amount which is sensitive to the square of the neutrino mass, but relatively insensitive to the uncertainty in the pion mass. 


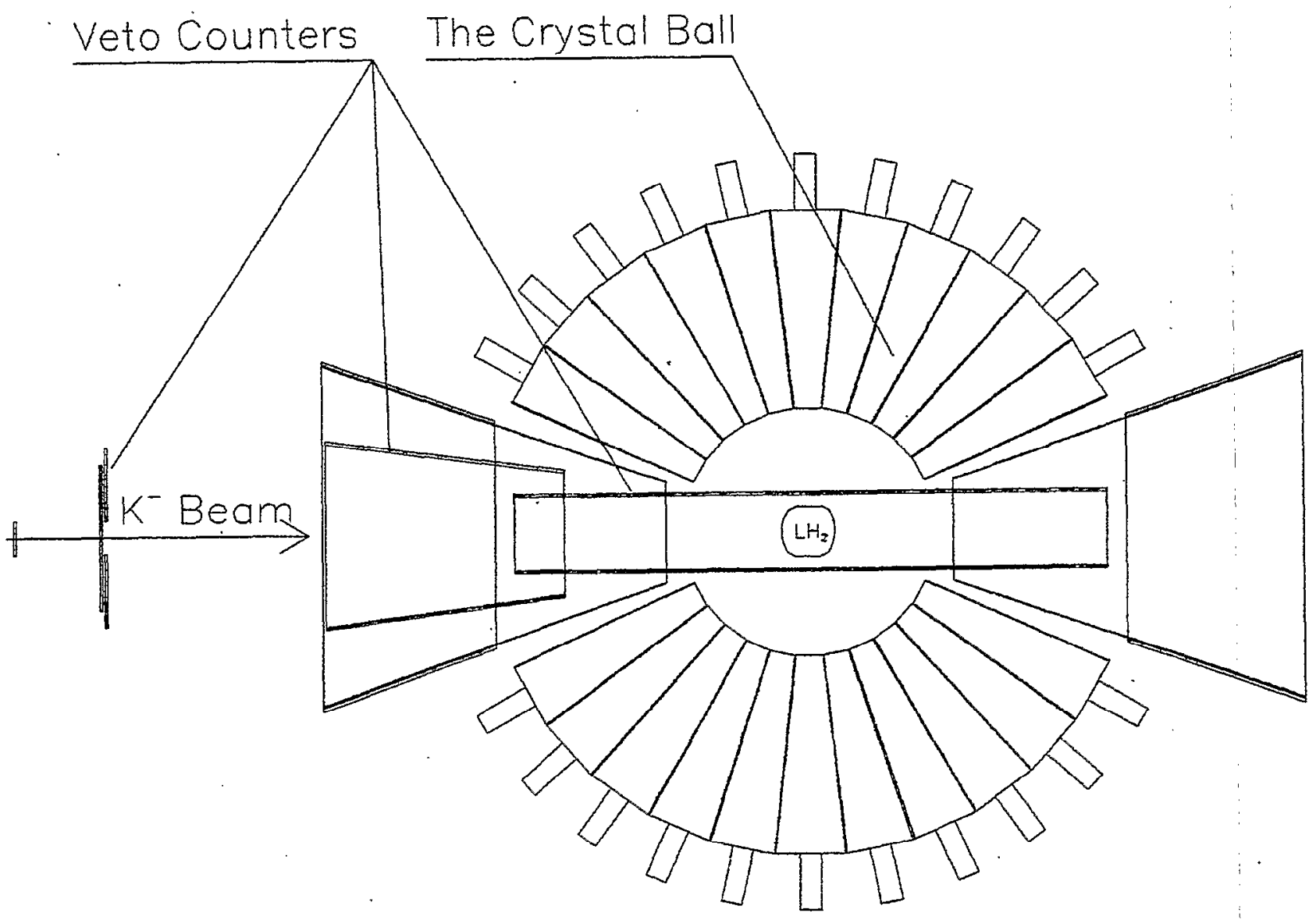




\section{EXPERIMENT 953 - NEUTRAL HYPERON SPECTROSCOPY WTTH THE CR YSTAL BALL}

\section{SPOKESPERSON: D. M. MANLEY, B.M.K. NEFKENS, H. SPINKA}

- Abilene Christian University - L. D. Isenhower

- Argonne National Laboratory - H. M. Spinka

- Arizona State University - J. R. Comfort

- George Washington University - W. J. Briscoe

- Kent State University - D.M. Manley

- Petersburg Nuclear Physics Institute - V.V. Abaev, V.S. Bekrenev, N.G. Kozlenko, S.P. Kruglov, A.A. Kulbardis, I.V. Lopatin

- Rudjer Boškovic Institute - I. Šlaus, I. Supek, A. Švarc

¿ University of California - B.M.K. Nefkins, S.N. Prakhov, J.W. Price, A.B. Starostin, W.B. Tippens

- University Colorado - R.J. Peterson

- University Karlsruhe - H.M. Staudenmaier

- University Maryland - D.C. Peaslee

- University Uppsala - H. Calen, S. Kullander, A. Kupsc, B. Morosov

- Valparaiso University - D. Grosnick, D.D. Koetke, R.W. Manweiler, T.D.S. Stanislaus

The spectroscopy of hyperons is a fundamental testing ground for nonperturbative QCD models, but ne that has been poorly studied. This proposal plans to perform measurements of absolute differential cross sectons for $\mathrm{K} p \rightarrow \gamma \Lambda, \mathrm{K}^{-} p \rightarrow \gamma \Sigma^{0}, \mathrm{~K}^{-} p \rightarrow \pi^{0} \Lambda, \mathrm{K}^{-} p \rightarrow \pi^{0} \Sigma^{0}, \mathrm{~K} p \rightarrow \mathrm{K}_{\mathrm{s}}^{0} n$, and $\mathrm{K} p \rightarrow \eta \Lambda$ in the momentum rante $500-750 \mathrm{MeV} / \mathrm{c}$. These measurements will complete the program originally approved as Phase $\mathrm{A}$ of AGS E914. 


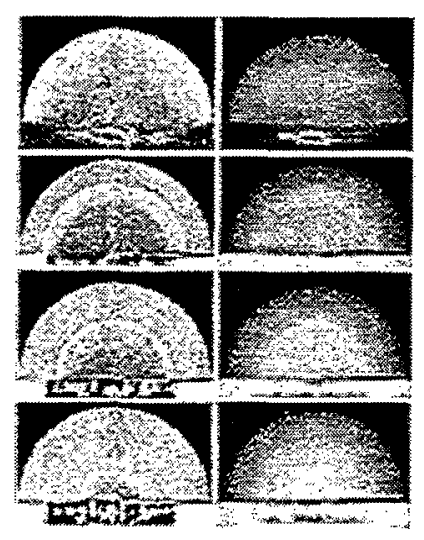

This photo shows a detonation wave at four different times in a high-explosive assembly. These exposures correspond to $0.99,1.90,2.50$, and $3.25 \mathrm{msec}$ (top to bottom) after detonation. The detonation wave is clearly evident in the radiographs as it propagates from the detonator to the outer surface of the explosive materials. The images were recorded on a phosphor image plate that allows one image per shot. An active camera system has now been installed and used to capture up to six frames in the time of a single high-explosive detonation. Future detector development is expected to provide the ability to take thousands of frames during the explosion to produce a "motion picture" of the event. 


\section{EXPERIMENT 955 - PROTON RADIOGRAPHY AT THE AGS IN THE U-LINE}

\section{SPOKESPERSENS: C.L.MORRIS, E. P. HARTOUNI}

- Bechtal Nevada - R. T. Thompson, R. P. Liljestrand, D. V. Morgan, A. Whiteson

- Brookhaven National Laboratory - G. Green, A. Hanson, J. Scaduto

- Lawrence Livermore National Laboratory - E. Ables, M. B. Aufderheide, P. D. Barnes Jr., E. P. Hartouni, H-S. Park, R. A. Soltz, L. Wiley, D. M. Wrigth

- Los Alamos National Laboratory - K. R. Alrick, K. L. Buescher, D. J. Clark, C. J. Espinoza, J. J. Gomez, N. T. Gray, G. W. Hart, M. Y. Hockaday, G. E. Hogan, N. S.P. King, A. R. Mathews, J.B. McClelland, K. B. Morley, C. L. Morris, C. T. Mottershead, K. H. Mueller, M. M. Murrag, P. D. Pazuchanics, J.S. Sarracino, A. Saunders, M. D. Wilke, J. D. Zumbro

Since experiment 933 was originally proposed, several additional questions have arisen about using protons for advanced radiography. This experiment program will include measurements on a wide range of step wedges to validate the separation of Coulomb and nuclear scattering using different collinators to demonstrate material $\mathrm{DD}$. These measurements will be made using both active detectors (cameras) and image plates (to ensure sufficient range). Data will be taken with the full range of available collimators for completeness. Domonstrations of material ID on classified thick objects will also be done. Background characterizaton and mitigation for the active cameras will be done. Sources and shielding techniques to reduce the star background in the CCD cameras will be studied. Data will be taken at a number of angles on suitable test objects aimed at providing input to help define the number of axis needed for an AHF. These data will probablybe taken on both image plates and with active cameras, with a greater emphasis on the active camera data in order to most efficiently use the beam time. 
EXPERIMENT 956

Home Page 


\title{
EXPERIMENT 956-SPALLATION NEUTRON STUDIES AT THE AGS
}

\author{
SPOKESPERSENS: J. B.HASTINGS, G. BAUER, N.WATANABE
}

- Argonne National Laboratory - J.M. Carpenter

- Brookhaven National Laboratory - J. B.Hastings

- Forschungszentrum Centre - Julich - H. Conrad

- Japan Atomic Energy Research Inst. - N. Watanabe

- Los Alamos National Laboratory - G. Russell

- Olak Ridge National Laboratory - J. Haines

- Paul Scherrer Inst. - G. Bauer

There is a worldwide effort in the planning, design and construction of the next generation neutron sources. The focus is on spallation sources with average powers of up to $5 \mathrm{MW}$ and single pulse energies of 100 kjoules. The critical design issues center around the heavy metal target. All of the present design studies are considering liquid mercury as a first choice. The AGS is a unique resource to study in detail neutron production, pressure wave mitigation and other important issues in target design. It is the only proton source in the world with the energy per pulse equal to the proposed future sources. The initial studies on a $\mathrm{Hg}$ target system have confirmed the unique role of the AGS. We propose to continue the measurements of the presusre wave behaviour under peak power loading and evaluate proposed mitigation measures. Another critical area is advanced moderator performance as well as materials studies for moderator containment. To carry out these studies it is important to develop a solid target that is passively safer as a neutron source. This target will be tested and then used in the second and third years of the program. We will study a novel moderator (methane pellet bed) and slab moderator configurations. These efforts will complete the establishment of a spallation neutron test facility capable of the full range of R\&D from the target through the moderator and up to and including advance detector development. 
Home Page http://bnlstb.bio.bnl.gov/biodocs/nasa/nasa ags.htmlx

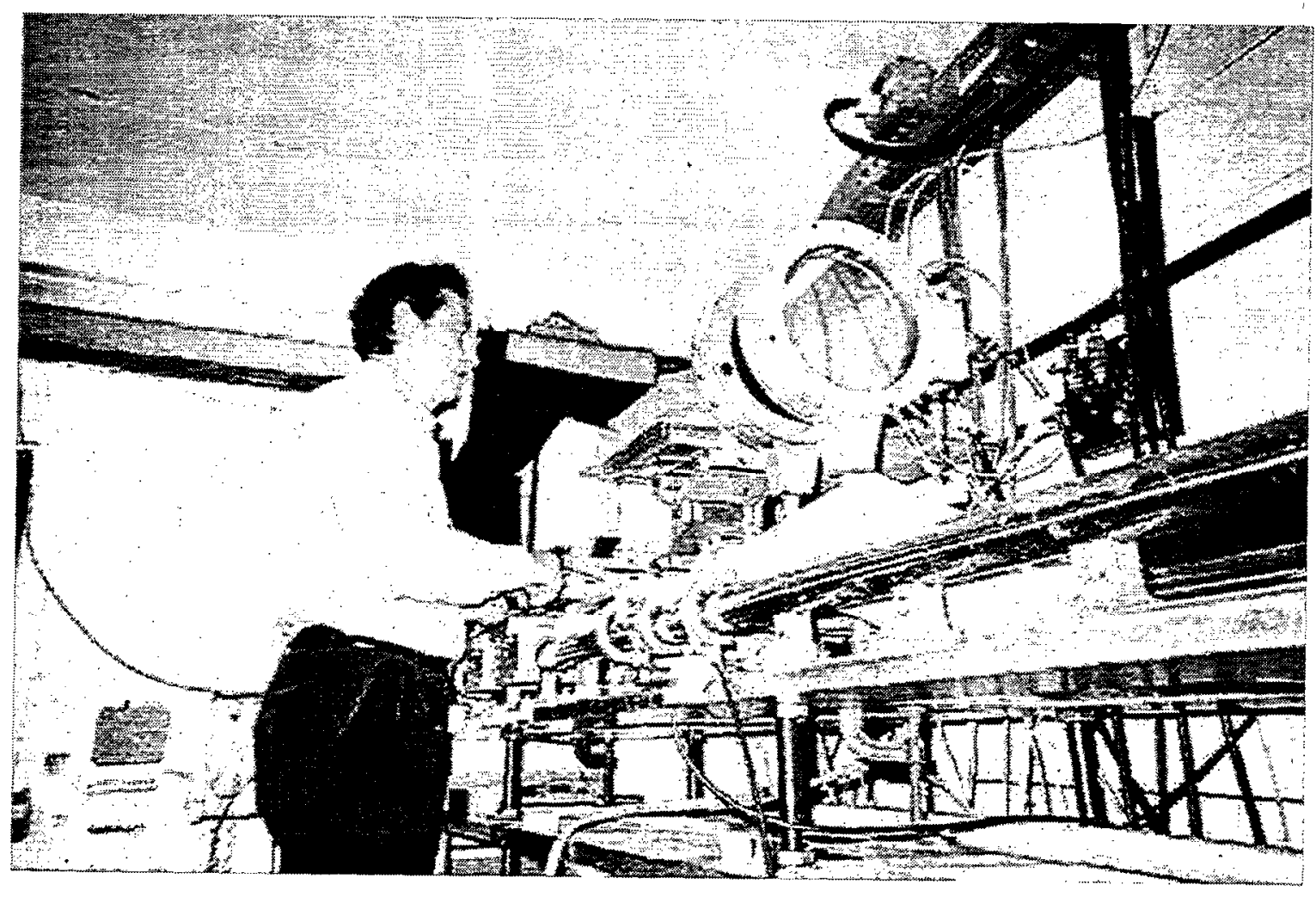

Dr. Mauro Belli setting up a sample to be exposed to iron ions 


\section{EXPERMENT 957-NASA RADIOBIOLOGY}

\section{SPOKESPERSON: M. VAZQUEZ}

- Brookhaven National Laboratory - R. Setlow, B. Sutherland, M. Vazquez

- Case Western Reserve University - H. Evans

- Colorado University - J. Bedford, C. Waldren

- Columbia University - T. K. Hei

- Human Nutrition Research Center on Aging - J. Joseph

- John Hopkins Medical Inst. - J. Dicello, D. Huso

- Lawrence Berkeley Laboratory - H. Barcellos-Hoff, J.Miller, P. Cooper, A. Kronenberg, B. Rydberg

- Loma Linda University - L. Green, G. Nelson

- NASA Headquarters - W. Schimmerling

- NASA Johnson Space Center - F. Cuccinota

- National Inst. of Health, Rome Italy - F. Antonelli, M. Belli, G. Simone

- NSCORTLBNL-CSU - J. Kinnison, D. Stephens

- New York University Medical Center - F. Bums

- Prairie View A\&M University - R. Wilkins

- Texas A\&M University - L. Braby, J. Lupton

- University California - San Fran. - B. Fouladi, J. Murnane

- University "Federico II", Napoli-M. Durante

- University of Maryland - B.Rabin

- University Pennsylvania - J. Gerwitz

- University of Rome, Thor Vergara - V. Bidoli, M. Cosolino, L. Narici, W.Sanita

- University of Texas Health Sciences - M. Natarajan

- University Tokyo - A. Sima

During the Winter of 2000 a series of radiobiologial and physics experiments were performed using BNL's AGS to accelerate iron ion beams. These experiments were part of the seventh consecutive run sponsored by NASA's Space Radiation Program (SRHP) heavy ion radiobiology research program at BNL.

The AGS provided iron beams with an enery of $1 \mathrm{GeV} /$ nucleon $(1.046 \mathrm{GeV} / \mathrm{nucleon}, \mathrm{LET}: 148 \mathrm{keV} / \mu \mathrm{m})$, for biology and physics experiments. The dose/rates used were as low as $10 \mathrm{cGy} / \mathrm{min}$ and as high as $15 \mathrm{~Gy} / \mathrm{min}$. The spill rate employed was $30 \mathrm{spills} / \mathrm{min}$ with a duration of $500-600 \mathrm{msec} / \mathrm{spill}$. The spill fluence was (particles/spill) $1 \times 10^{8}$ (max) and $1.5 \times 10^{5}$ (min). The intensities (particles $/ \mathrm{cm}^{2} / \mathrm{sec}$ on target) used during the run were $1 \times 10^{8}$ (max) and 400 (min). A $7.5 \mathrm{~cm}$ diameter beam spot was employed as a nominal spot for the majority of the exposures. For larger samples (animals), an elliptical spot was used (up to $9 \mathrm{~cm}$ ).

Radiobiological experiments employed cells, tissues, and intact specimens, which required a complex coordination and planning of their respective logistic support. Biological studies used human, mouse, rat and hamster cell lines, humanhamster hybrid cell lines, tumor cell lines and intact specimens (rodents and fish). Physics experiments involved the exposure of solid state detectors and spacecraft materials. 
Home Page http://bmkn8.phusics.ucla.edu/Crustalbal//crustalballhhtml 


\section{EXPERIMENT 958 - PION CHARGE-EXCHANGE CROSS-SECTIONS AT LOW ENERGIES}

\section{SPOKESPERSENS: M.E. SADLER, J.COMFORT}

- Abilene Christian University - L. D. Isenhower

- Argonne National Laboratory - H. M. Spinka

- Arizoma State University - J. R. Comfort, K.Craig, T. Ramirez

- George Washington University - W. J. Briscoe, A. Shafi

- Karlsruhe University - H. Staudenmeier

- Kent State University - D. M. Manley, J. Olmsted

- Petersburg Nuclear Physics Institute - V.V. Abaev, V.S. Bekrenev, N.G. Kozlenko, S.P. Kruglov, A.A. Kulbardis

- Rudjer Boškovic Institute -I. Supck

- University of California - B.M.K. Nefkins, N. Phaisangitisakul, S.N. Prakhov, J.W. Price, A.B. Starostin

- University Colorado - R.J. Peterson

- University Karlsruhe - H.M. Staudenmaier

- University Maryland - D.C. Peaslee

- University Regina - N. Knecht, G. Lolos, Z. Papandreou

- University Uppsala - H. Calen, A. Kupsc, T. Johanson, U.Wiedner

- Valparaiso University - D. Groznick, D. D. Koetke, R.Manweiler, S. Stanislaus

The experiment will measure the cross-section angular distributions for the $\pi \mathrm{p} \rightarrow \pi^{0} n$ reaction with the Crystal Ball spectrometer for momenta between $50-180 \mathrm{MeV} / \mathrm{c}$. The motivations for this is to provide accurate data to explore isospin invariance in the $\pi \mathrm{N}$ system, to supercede existing data that does not agree well with partial-wave analysis (PWAs) and to complement differential cross-section and analyzing power date for $\pi^{+} p \rightarrow \pi^{+} p$ and $\pi^{-} p \rightarrow \pi^{0} n$. By providing high-quality results for the chargeexchange reaction, the amplitudes for $\pi \mathrm{N}$ interactions can be defined more precisely and enable more reliable extractions of the up-down quark mass difference and the sigma term. 
EXPERIMENT 960

Home Page

Beam: A3 


\section{EXPERIMENT 960-NASA RADIOBIOLOGY}

\section{SPOKESPERSON: M. VAZQUEZ}

- Alabama A\&M University - P. Kale

- Brookhaven National Laboratory - B. Sutherland, M. Vazquez

- Case Western Reserve University - H. Evans

- Colorado University - J. Bedford, C. Waldren

- Columbia University - T. K. Hei

- John Hopkins Medical Inst. - J. Dicello

- Lawrence Berkeley Laboratory - H. Barcellos-Hoff, J.Miller, P. Cooper, A. Kronenberg

- Los Alamos National Laboratory - D. J.Chen

- Pacific Northwest National Laboratory - N. Metting

- Texas A\&M University - R. R. Sinden

- University of Maryland - B.Rabin

- Washington State University - A. L. Brooks

This is a continuing program of experiments in radiobiology, funded by the National Aeronautics and Space Agency (NASA) as part of their space-related research efforts in the life sciences. 


\section{List of Publications for $C-A D$ Experiments}

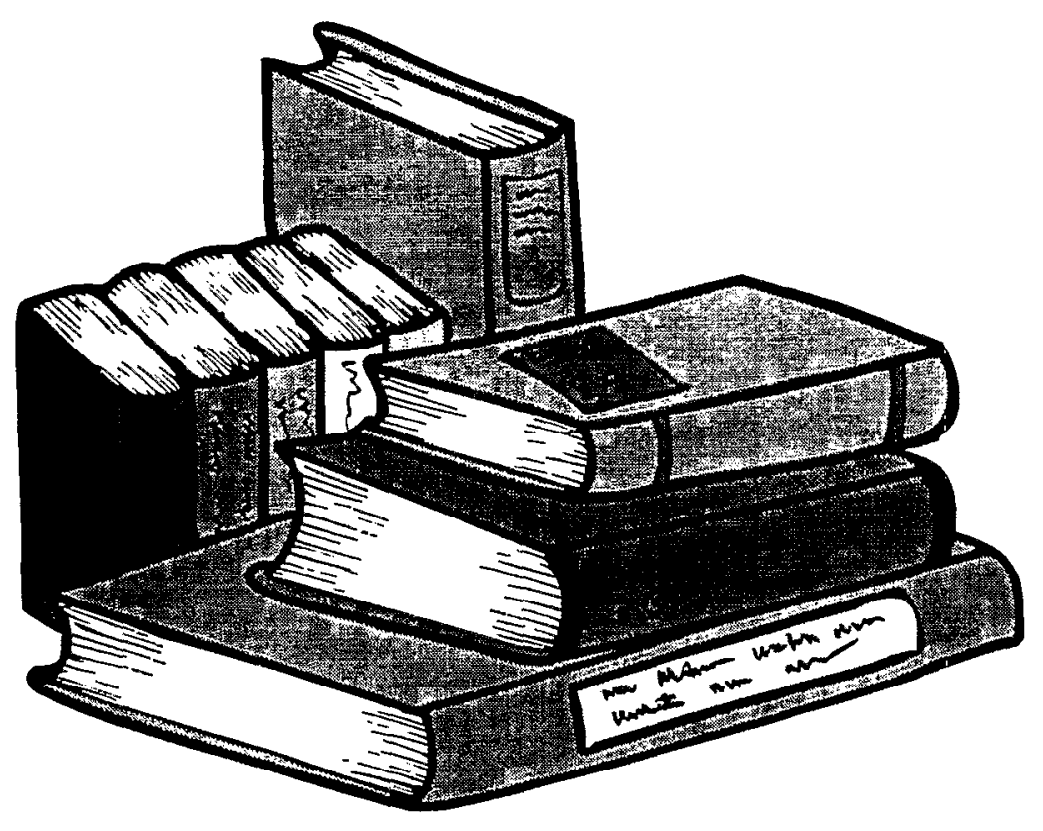




\section{Publications - AGS Experiments 1982 - 2001}

This listing was originally prepared using the SLAC data base SPIRES; we now rely on the experimenters themselves to supply us with information. It is easy to miss publications in such a wide search and we apologize for any left out or misidentified. Please let us know about these as well as keeping us posted on. your recent publications of $C$-AD experiments.

\section{BRAHMS}

F. Videbaek, The BRAHMS experiment at RHIC, Nucl. Phys. A566 (1994)(Quark Matter '93).

F. Jundt, et al., Time projectio chambers for ultra-relativistic heavy-ion experiments with amid-rapidity spectrometer", Nucl. Phys. A566 (1994) (Quark Matter '93).

B. Moskowitz, Hadron distributions at higher rapdidity using the BRAHMS forward spectrometer, Physics w/ the collider detectors at RHIC and the LHC, Proc. of Pre-Conf. Workshop (Quark Matter 95).

D. Beavis, Mid-rapidity measurements with the BRAHMS spectrometer, Physics w/ the collider detedtros at RHIC and the LHC, Proc. of the pre-Conf. Workshop (Wuark Matter 95).

R. Debbe, et al., In-beam tests of a ring imaging Cerenkov detector with a multianode photomultiplier readout, Nucl Inst. Meth. A362 (1995).

R. Debbe, et al., the ring imagining Cherenkov detector for the BRAHMS experiment. Nucl. Instrum. Meth. A371 (1996) 327-329.

F. Videbaek, The BRAHMS experiment at RHIC, proc. of the RHIC summer-study 1996.

F. Videbaek, the BRAHMS experiment at RHIC, status and goals, Proc. of "workshop on particle distributions in hadronic and nuclear reactions", UIC, June 10-12, 1998.

F. Videbaek, The BRAHMS experiment at RHIC, staus and first years physics, Proc. of Heavy ion mini symposium, Atlanta, (1999).

\section{JPHENIX}

R. Seto, PHENIX, invited talk at $2^{\text {nd }}$ Intl. Conf. On Physics and Astrophysics of Quark-Gluon Plasma, Calcutta, India, January, 1993.

N. Saito, Spin physics with PHENIX detector system at RHIC, Talk presented at Adriatico Research Conf., "Trends in Colider Spin Physics", ICTP, Trieste, Italy, Dec. 1995 and at the RIKEN Symposium "Spin Structure of the Nucleon", RIKEN, Wako, Saitama, Japan, December 1995.

J. T. Mitchell (for the PHENIX collaboration), An overview of the PHENIX experiment at RHIC, Proc. of $14^{\text {th }}$ Intl. Conf. On Particles and Nuclei, World Scientific, p. 433 (1996), ANIC Conf. 1996.

D. P. Morrison, The PHENIX experiment at RHIC, (IV-E-44), published in proc. of $13^{\text {th }}$ Intl. Conf. on UltraRelativistic Nucleus-Nucleus Collisions, Tsukuba, Japan, Quark Mater '97, Nuclear Physics A638 (1998) 575c, Dec. 1-5, 1997.

N. Saito, Spin physics with PHENIX detectors, (IV-E-17), published in the proc. of the $13^{\text {th }}$ Intl. Conf. on UltraRelativistic Nucleus-Nucleus collisions. Tsukuba, Japan, Quarkk Matter '97, Nuclear Physics A638 (1998) 575c, Dec. 1-5, 1997.

Y. Miake, PHENIX experiment and signatures of $Q G P$, Intl. Workshop on Contemporary Nuclear Physics by Korea Detector Laboratory, Korea U., Seoul, Korea, Apr 30-May 2, 1998.

K. Read, The PHENIX experiment, submitted to Kluwer Academic Press, New York 1999. 
$\mathrm{K}$. Adcox, et al., Centrality dependence of charged particle multiplicity in Au-Au collisions at $\sqrt{\mathrm{S}_{\mathrm{NN}}}=130 \mathrm{GeV}$, Submitted to Phys. Rev. Lett, December 2000.

\section{PHOBOS}

W. Busza, Low Pt physics and compact detectors at RHIC and LHC, in Particle Production I Highly Excited Matter, Plenum Press Pub., pp.149-157 (1993).

A. Wyslouch, The PHOBOS experiment at RHIC and AGS, Nuclear Phys. A, pp.305c0310c, (1994).

W. Tsay, J. Hong, A. Chen, W.T. Lin, C. Hsu, S. Jan, C. Kuo, Improved process of fabrication AC-coupled silicon micro-strip sensors, Nucl. Inst. and Meth. A351, pp. 463-465 (1994).

K. Wozniak, PHOBOS experiment at RHIC, proc. XXV Intl. Symposium on the Multiparticle Dynamics, pp10, (1995).

R. Betts, Current status of PHOBOS at RHIC publication, Advances in Nuclear Dynamics 2, Bauer and Estfall eds., Plenum Press, NY, pp. 225-232 (1996).

W. T. Linn, et al., Development of a double metal, AC-coupled silicon pad detector. The silicon detector for the PHOBOS experiment at RHIC, Nucl. Instr. Meth. A389, 415 (1997).

B. Black, et al., Charged particle multiplicity near mid-rapidity in central Au+Au collisions at $\sqrt{ } s=56$ and 130 AgeV, Physics Review Lett. 85 pp. 3100 (2000).

R. Bindel, eta 1., Array of scintillator counters for PHOBOS at RHIC, Nucl. Instr. Meth. (2001).

R. Nouicer, et al., Silicon pad detectors for the PHOBOS experiment at RHIC, Nucl. Inst. Meth. A461, pp.143-149 (2001).

\section{SPIN}

T. Roser, Polarized proton beams, proc. PAC '95, pp. 3154

A.N. Zelenski et al., The TRIUMF high-current DC optically-pumped polarized $H$ ion source, proc. PAC '95, pp. 864.

M. Okamura, et al., Three dimensional field analysis of helical snake magnets for RIIIC, proc. PAC '96, pp. 3359.

A.N. Zelenski et al., High-current, optically-pumped, polarized $H$ ion source development for high energy accelerators, proc. PC '96, pp. 1530.

H. Wu, et al., Spin dynamics study for RHIC, proc. Pac '96, pp. 974.

M. Syphers, et al., Helical dipole magnetrs for polarized protons in RHIC, proc. PAC '97, pp. 3359.

E. Willen et al., A helical magnet design for RHIC, proc. PAC '97, pp. 3362.

W. Fischer and M. Okamura, Parameterization and measurements of helical magnetic fields, proc. PAC '97, pp. 341.

H. Huang, et al., Overcoming weak intrinsic depolariziang resonances with energy-jump, proc. PAC '97, pp. 1460.

M. Okamura, et al., Three dimensional field analysis of helical magnet for RHIC Siberian snake, proc. PAC '97, pp. 3431.

T. Tominaka, et al., Analytical field calculation of helical dipole magnets for RHIC, proc. PAC '97, pp. 3437. 
M. Bai et al., Overcoming intrinsic spini resonances by using an C dipole, proc. PAC '97, pp. 1478.

N. Tsoupas, et al., Transfer of a polarized proton beam from AGS to RHIC, proc. PAC '97, pp. 207.

A.U. Luccio et al., Spin tracking in RHIC, proc. PAC'97, pp. 92.

T. Tominaka, et al., Intrinsic limit of field homogeneity of helical dipoles, proc. PAC '98, pp.567.

T. Katayama et al., Field calculation and measurement of a full-length snake magnet for RHIC, proc. EPAC '98, pp. 2005.

G. Dutto et al., Development of high-current polarized $H$ ion source at TRIUMF, proc. EPAC '98, pp. 290.

Y.K. Batygin and T. Katayama, Spin depolarization by the beam-beam effect, proc. EPAC '98, pp. 1026.

T. Roser, Acceleration of polarized protons to high energy, proc. PAC '99, pp. 26.

A.N. Zelenski et al., Optically-pumped polarized $H$ ion source for RHIC and HERA colliders, proc. PAC '99, pp. 106.

M. Xiao et al., DA method and symplectification for field map generated matrices of Siberian snake in RHIC, proc. PAC' 99 , pp. 404.

H.Huang et al., A p-carbon CNI polarimeter for RHIC, proc. PAC '99, pp. 471.

A.Luccio et al., New capabilities of the spin tracking code SPINK, proc. PAC '99, pp. 1578.

J. Alessi et al., Design of a $35 \mathrm{keV}$ LEBT for the new high intensity OPPIS at BNL, proc. PAC '99, pp. 1964.

E. Willen et al., Construction of helical magnets for RHIC, proc. PAC `99, pp. 3161.

T. Tominaka et al., Rotation angle of a helical dipole, proc. PAC '99, pp. 3164.

C.Parker et al., Design of an AC-dipole for use in RHIC, proc. PAC '99, pp. 3336.

STAR H. Wieman, et al., Recent developments on the STAR detector system at RHIC, Nuclear Physics A, vol, 638, pp. 559-564 (1998).

K. H. Ackermann, et al., The STAR time projection chamber, Nuclear Physics A, Vol. 661, pp. 681-685, (1999)

K. H. Ackermann, et al., Elliptic flow in $A u+$ Au collisions at $\sqrt{\mathrm{S}_{\mathrm{NN}}}=130 \mathrm{GeV}$, Phys. Rev. Lett. Vol. 86, 3, pp. $402(2001)$.

723 D.W. Hertzog, et al., Precision measurement of the magnetic moment of the $\Sigma$; Phys. Rev. Lett. 51, 1131 (1983).

734 L.A. Ahrens, et al., New limit on the strength of mixing between $v_{\mu}$ and $v_{e}$. Phys. Rev. D31, 2732 (1985).

L.A. Ahrens, et al., Measurement of the ratio of cross-sections for neutrino and anti-neutrino-scattering from electrons, Phys. Rev. Letts. 54, 18 (1985).

L.A. Ahrens, et al., Determination of the neutrino fluxes in the Brookhaven wide-band beams, Phys. Rev. D34, 75 (1986) .

L.A. Ahrens, et al., Precise determination of $\sin ^{2} \theta_{w}$ from measurements of the differential cross-sections for $v_{\mu}$ $p \rightarrow v_{\mu} p$ and $\bar{v}_{\mu} p \rightarrow \bar{v}_{\mu} p$, Phys. Rev. Letts. 56, 1107 (1986). 
L.A. Ahrens, et al., Measurement of $v p$ and $\bar{v}$ p elastic scattering, Phys. Rev. D35, 785 (1987).

L.A. Ahrens, et al., A massive fine-grained detector for the elastic reactions induced by neutrinos in the GeV energy region, Phys. Rev. D35, 785 (1987).

745 M. May, et al. 3d-3p Transition in $\left(\mu^{-} \mathrm{He}^{4}\right)^{+}$, Proc. of Workshop on Fundamental Muon Physics: Atoms, Nuclei and Particles, Los Alamos (1986).

A. Blaer, et al., Measurement of $K x$-rays from muonic helium formed in a low density target in an intense pulsed muon beam, Phys. Rev. A. (1989).

747 A. Etkin, et al., The reaction $\pi^{-p} p \rightarrow \phi \phi n$ and evidence for glueballs, Phys. Rev. Lett. 49, 1620 (1982).

S. J. Lindenbaum, Comments on glueballs, multi-quark states and the OZI rule, Phys. Lett. 131B, p. 221-223 (1983).

S. J. Lindenbaum, The discovery of glueballs, surveys in high energy physics. Vol. 4, p. 69-126, John M. Charap, Editor (Harvest Academic Publishers, London, 1983).

S. J. Lindenbaum, Production of glueballs, Comments on Nuclear and Particle Physics 13, No. 6, p. 285-311 (1984).

S. J. Lindenbaum and H. J. Lipkin, Comments on the reaction $\pi^{-} p \rightarrow \phi \phi n$ and glueballs, Phys. Lett. 149B, p. 407 (1984).

A. Etkin, et al., Observation of three $2^{++}$resonances in the glueball-enhanced channel $\pi{ }^{\circ} \pi \rightarrow \phi \phi n$, Phys. Lett. B165, 217 (1985).

S.J. Lindenbaum and R.S. Longacre, The glueball resonance and alternative explanations of the reaction $\pi^{-}$ $p \rightarrow \phi \phi n$, Phys. Lett. 165B, 202 (1985).

748 . P.H. Hansen, et al., Spin effects in pp elastic scattering at $28 \mathrm{GeV/c}$, Phys. Rev. Lett. 50, 802 (1983).

749 J.K. Black, et al., Measurements of the CP-non-conservation parameters $\varepsilon^{\prime} / \varepsilon^{\prime}$, Phys. Rev. Lett. 54, 1628 (1985).

754 P. Kammel, et al., First observation of hyperfine transitions in muonic deuterium atoms via resonant $D \mu D$ formation at 34-K, Phys. Lett. 112B, 319 (1982).

W.H. Bertl, et al., Hyperfine transition of $\mu d$ atoms in liquid hydrogen-deuterium mixture, Kerntechnik 43, 184 (1983).

755 G.C. Blazey, et al., Hard scattering with exclusive reactions: $\pi^{\circ} p$ and meson production, Phys. Rev. Lett. 55, 1820 (1985).

S. Heppelmann, et al., Decay distribution of high-transverse-momentum $\rho$ mesons, Phys. Rev. Lett. 55, 1824 (1985).

766 M. Church, et al., Exclusive Cascade Minus Production in 15-28 GeV Neutron-Proton Interactions, invited paper presented at the 23rd Intl. Conf. on High Energy Physics, Berkeley, CA, July 1986.

G. Tzanakos, Search for neutrino oscillations, Proc. of BNL Neutrino Workshop, M. Murtagh, editor (1987).

E. P. Hartouni, et al., Some preliminary results from BNL E766 on $p p \rightarrow p p K_{s}^{\circ} K \pi$, contribution to the BNL Workshop on Glueballs, Hybrids and Exotic Hadrons, August 29-September 1, 1988. 
E.P. Hartouni, et al., A new technique for on-line and off-line high speed computation, IEEE Transactions on Nuclear Science, 36, No. 5, 1480, October 1989.

L. Wiencke, et al., Photon production in fully reconstructed pp collisions at $27.5 \mathrm{GeV} / \mathrm{c}$, presented at the Pittsburgh Workshop on Soft Lepton Pair and Photon Production, September 6-8, 1990.

J. Felix, et al., Study of $\Lambda^{0}$ polarization in $p p \rightarrow p \Lambda^{0} K^{+} \pi^{+} \pi^{*} \pi^{+} \pi^{-}$at $27.5 \mathrm{GeV}$, Phys. Rev. Letts. 76, 22 (1996)

767 T. Armstrong, et al., A search for narrow states in anti-neutron proton total and annihilation cross-sections near anti-N N threshold, Phys. Lett. B175, 383 (1986).

769 R.S. Longacre, et al., A measurement of $\pi^{-} p \rightarrow \pi^{-} p \rightarrow K_{s}^{0} K_{s}^{o} n$ at $22 \mathrm{GeV} / \mathrm{c}$ and a systematic study of the $2^{++}$ meson spectrum, Phys. Lett. 177B, 223 (1986).

771 S.U. Chung, et al., Spin and parity analysis of KK $\pi$ system in the $D$ and E/Lota regions, Phys. Rev. Lett. 55, 779 (1985).

D.R. Reeves, et al., Spin-parity analysis of $p p \rightarrow E$ (1420)X, Phys. Rev. D34, 1960 (1986).

A. Birman, et al., Partial-wave analysis of the $K^{+} K^{0} \pi{ }^{*}$ system, Phys. Rev. Lett. 61,1557 (1988).

H. Piekarz, Searches for dibaryons of strangeness -1. NP A463 (1987) 205c, Nucl. Phys. A450, 85c, (1986).

Shlomit Tarem, Physics Department, Brandeis University, 1987. PhD Thesis.

Y. D. He, P. B. Price, and W. T. Williams, Interactions of projectile fragments at $14.5 \mathrm{~A} \mathrm{GeV}$ : A search for anomalons, Phys. Lett. B 252, 331-335 (1990).

774 R.E. Chrien, E.V. Hungerford, and T. Kishimoto, Continuum effects and the interpretation of $\Sigma$-Hypernuclei. Phys. Rev. C35, 1589 (1987).

Reyad Sawafta, et. al., Do narrow sigma hypernuclear states exist? Nucl. Phys. $\Lambda 585$ 103-108 (1995).

N.J. Baker, et al., Search for short-lived neutral particles emitted in $K^{+}$decay, Phys. Rev. Lett. 59, 2832 (1987).

C. Campagnari, et al., Search for the Decay $K^{+} \rightarrow \pi^{+} \mu^{+} e^{-}$, Phys. Rev. Lett. 61, 2062 (1988).

D.M. Lazarus, et al., AIP Conf. Proc. 176, 3rd Intl. Conf. on the Intersections Between Particle and Nuclear Physics, G. Bunce, editor (1988).

A.M. Lee, et al., Improved limit on the branching ratio of $K^{+} \rightarrow \pi^{+} \mu^{+} e^{*}$, Phys. Rev. Lett. 64,165 (1990).

C. Alliegro, et al., Rare $K^{+}$decays in flight: latest results and future plans, particles and fields 1991, The Vancouver Meeting, 587 (1991).

778 B.C. Stringfellow, et al., Accelerator internal target experiments using a supersonic gas jet, NIM A251, 242 (1986).

779 Y.Y. Chu and M.C. Zhou, Identification of ${ }^{233}$ Ac, Phys. Rev. C28, 1379 (1983).

780

H.B. Greenlee, et al., A search for $\mathrm{K}_{\mathrm{L}}^{0} \rightarrow \mathrm{\mu}^{+} \mathrm{e}$ and $\mathrm{K}_{\mathrm{L}}^{0} \rightarrow \mathrm{e}^{+} \mathrm{e}^{*}, B N L 40452$.

E. Jastrzembski, et al., Phys. Rev. Lett. D61, 2300 (1988).

S.F. Schaffner, et al., Phys. Rev. D39, 990 (1989). 
781 R. E. Chrien, et al., Search for radiative transitions in the hypernucleus ${ }_{A}^{10} \mathrm{~B}$, Phys. Rev. C41, 1062-1074 (1990). M. May et al.,First observation of the $p_{1} \rightarrow s_{1} \gamma$-ray transition in ${ }_{\wedge}^{13} \mathrm{C}$, Phys. Rev. Lett. 78, 4343-4346 (1997).

782 K.A. Brown, et al., Measurement of $p+p \rightarrow p+p$ with a $16.5 \mathrm{GeV} / c$ polarized proton beam, Phys. Rev. D31, 3017 (1985).

G. R. Court, et al., Energy dependence of spin effects in $p \uparrow \rightarrow p \uparrow \rightarrow p+p$, Phys.Rev.Lett.57, 507 (1986).

D.G. Crabb, et al., Measurement of spin effects in $p+p \rightarrow p+p$ at $18.5 \mathrm{GeV} / c$, Phys. Rev. Lett. 60, 2351 (1988).

785 Y.I. Makdisi, Experimental results on spin physics at the AGS, 7th Int. Symp. on High Energy Spin Physics, Protvino, USSR, September 22, 1986.

787 S. Ahmad, E.W. Blackmore, D.A. Bryman, J. Cresswell,T. Numao, Central drift chamber for rare kaon decay spectrometer, IEEE Trans. Nucl. Sci. NS-33, 178 (1986).

R. C. Strand, Status of a study of the decay $K^{+} \rightarrow \pi^{+} \bar{v} v$, Third Conf. on the Intersections Between Particle and Nuclear Physics, Rockport, Maine, Ed. Gerry M. Bunce, p. 866-73 (1988).

M.S. Atiya, et al., A study of $K^{+} \rightarrow \pi^{+} v \bar{v}$, IX European Symposium on Anti-proton-proton interactions and fundamental symmetries, Mainz, W. Germany, Sept. 5-10, 1988.

J.V. Cresswell, S.Ahmad, B.W. Blackmore, D.A. Bryman, N. Khan, Y. Kuno, T. Numao, A cylindrical drift chamber for the measurement of $K^{+} \rightarrow \pi^{+} v \bar{v}$, IEEE Trans. Nucl. Sci, 35, 460-463 (1988).

C. L. Woody, Aging effects in wire chambers operated at low pressure with TMAE and its effect on the use of $B a F_{2} T M A E$ calorimetry at the SSC, Proc., Workshop on Radiation Effects at the SSC, Berkeley,CA., Ed. M.G.D. Gilchriese, p. 207-14 (1988).

J. S. Frank and R. C. Strand, Construction and performance of a plastic scintillating fiber target for a rare kaon decay experiment. Proc. Workshop on Scintillating Fiber Detector Development for the SSC, Batavia, IL., Nov. 14-16, 1988, p. 361-382.

M. Atiya, M. Ito, J. Haggerty, C. Ng and F. W.Sippach, Waveform digitizing at $500 \mathrm{MHZ}$, Intern. Conf. on advanced Technology and Particle Physics, Como, Italy, June 13-17, 1988, Nucl. Instrum. and Meth., A279, 180-5 (1989).

M.S. Atiya, et al., Search for $K^{+} \rightarrow \pi^{+} v \bar{v}$, Proc., "Rare Decay Symposium," Vancouver, B.C., Nov. 1988, D. Bryman, J. Ng, T. Numao, and J-M. Poutissou, Editors, World Scientific, Singapore, p. 61-80, 1989.

M.S. Atiya, et al., Search for the rare decay $K^{+} \rightarrow \pi^{+} v \bar{v}$, Proc., Fourth Family of Quarks and Leptons: Second Intern. Symposium, Santa Monica, CA, Feb. 1989, Annals of the Academy of Sciences 578, 202-14 (1989).

M.S. Atiya, et al., A search for a light higgs boson in the decay $K^{+} \rightarrow \pi^{+} H, H \rightarrow \mu^{+} \mu^{-}$, Phys. Rev. Lett. 63, 2177-80 (1989).

P.D. Meyers, Brookhaven Experiment 787, the search for $K^{+} \rightarrow \pi^{+} v \bar{v}$, Proc., 24th Rencontres de Moroid Session on Electroweak Interactions and Unified Theories, Les Arcs, France, March 5-12, 1989.

M.A.Selen, Hunting for the rare decay $K^{+} \rightarrow \pi^{+} \mu^{+} \mu^{-}$, Princeton University DOE/ER-3072-49, January 1989.

M. Atiya, et al., Waveform digitizing at 500 MHZ, IEEE Trans. on Nucl. Sci. 36, 813-7 (1989). 
Daniel S. Akerib, A search for the rare decay $K^{+} \rightarrow \pi^{+} v \bar{v}$, Princeton University OE/ER-3072-64. January 1989.

C. L. Woody, P. W. Levy and J. A. Kierstead, Slow component suppression and radiation damage in doped BaF $F_{2}$ Crystals, IEEE Trans. on Nucl. Sci. 36, 536-42 (1989).

T. Numan, Status and future of the BNL E787 experiment, Proc. Workshop on K-Decay Experiments, KEK Report 89-7, 85 (1989).

M.S. Atiya, et al., Search for the decay $K^{+} \rightarrow \pi^{+} v \bar{v}$, Phys. Rev. Lett. 64, $21-4$ (1990).

M.S. Atiya, et al., Search for the decay $K^{+} \rightarrow \pi^{+} \gamma \gamma$, Phys. Rev. Lett. 65, 1188-91 (1990).

M.S. Atiya, et al., Search for the flavor-changing neutral current decay $K^{+} \rightarrow \pi^{+} v v$, Proc. 15th APS Division of Particles and Fields General Meeting, Houston, Texas, Jan. 3-6, 1990.

M.S. Atiya, et al., Search for the decay $K^{+} \rightarrow \pi^{+} v \bar{v}$, Phys. Rev. Lett. 64, $21-4$ (1990).

T. Numao, et al., Recent results on rare $K^{*}$ decays from BNL E787, Proc., Fourth Intl. Conf. on inter-sections between particles and nuclei, Nucl. Phys. A527, 727 (1991).

D.Bryman, et al., 500-MHZ transient digitizers based on GaAs CCDs, Proc., Symposium on Detector Research and Development for the Superconducting Supercollider, eds. T. Dombeck, V. Kelly, and G. P. Yost, World Scientific pp 505-507 (1991).

M. S. Atiya, et al., Upper limit on the branching ratio for the decay $\pi^{\circ} \rightarrow v \bar{v}$, Phys. Rev. Lett. 66, 2189-92 (1991).

M. Atiya, et al., Search for $K^{+} \rightarrow \pi^{+} v \bar{v}$ and other rare $K^{+}$decays, Nucl. Phys. A527, 727-730c (1991).

I. H.Chiang and L. Littenberg, CsI R\&D, Proc. KEK Workshop on Rare Kaon Decay Physics, Ibaraki-Ken, Japan, Dec. 1991, T. Shinkawa and S. Sugimoto eds. p. 303-309, 1992.

M.S. Atiya, et al., A detector to search for $K^{+} \rightarrow \pi^{+} v \bar{v}$. Nucl. Instr. \& Methods, A321, 129-151 (1992).

M.S.Atiya, et al., Search for the decay $\pi^{0} \gamma+X$. Phys. Rev. Lett. 69, 733-736, (1992).

M. Ito, et al., Search for $K^{+} \rightarrow \pi^{+} v \bar{v}$. Proc., Moriond Conf., Les Arcs, France (1993).

M. Atiya, et al., Search for the decay $K^{+} \rightarrow \pi^{+} v \bar{v}$. Phys. Rev. Lett. 70, 2521-4 (1993).

C. Zein, et al., A high-speed fastbus interface to VME. Real-Time Computing Appl. 1993. 103 (1993).

M.S. Atiya, et al., Search for the decays $K^{+} \rightarrow \pi^{+} v \bar{v}$ and $K^{+} \rightarrow \pi^{+} X^{0}$ for $150<\mathrm{M}_{\mathrm{x}}^{0}<250 \mathrm{MeV} / \mathrm{c}^{2}$, Phys. Rev. D48, R1 (1993).

M.S.Atiya, et al., An improved search for the decay, $K^{+} \rightarrow \pi^{+} v \bar{v}$. Phys. Rev. Lett. 70, 2521 (1993).

M. Burke, et al., E787 data acquisition software architecture, Conf. Record of the 8th Conf. on Real-Time Computer Applications in Nuclear, Particle, and Plasma Physics, Vancouver, June 8-10,1993, eds. D. Axen and R. Poutissou, p. 361-4 (1993).

J.S. Frank, Status and future plans for BNL experiment $787\left(K^{+} \rightarrow \pi^{+} v \bar{v}\right)$. Proc. of the Workshop on Future Directions in Particle and Nuclear Physics at Multi-GeV Hadron Beam Facilities, D. F. Geesaman, Editor, p. 42833, 1993. 
M. Burke, et al., E787 data acquisition software architecture, IEEE Trans. Nucl. Sci., NS-41, 131-4 (1994).

M. M. Ito, Results from BNL E787, a search for $K^{+} \rightarrow \pi^{+} v \bar{v}$. Proc. 1994 Workshop on Heavy Quarks Physics at Fixed Target, University of Virginia, Charlottesville, VA., October 1994, B. Cox, Editor, p. 359-364, 1994.

C. Witzig, Recent results on rare kaon decays from BNL E787, Proc. XXIX Rencontre de Moriond, Mar. 1995, J. Trân Thanh Vân, Ed., 361-5, (1994).

M. Kobayashi, et al., Yal0 $:$ Ce-Am light pulsers as a gain monitor for undoped CsI detectors in a magnetic field, Nucl. Instr. \& Method., A337, 3550-361 (1994).

J. Haggerty (for the E787 collaboration), Observation of $K^{+\rightarrow} \pi^{+} \mu^{+} \mu^{-}$, Proc. Of XVII Intl. Conf. on High Energy Physics, P. J. Bussey and I. G. Knowles, eds., 1341, (1995).

J. Roy, et al., Status of E787, Proc. Intersection between Particle and Nuclear Physics: Fifth Conf. held in'St. Petersburg, Fla., June 1994 ed. S. J. Seestrom, Los Alamos Natl. Laboratory (1995).

R. A. McPherson, Chasing the rare decay $K^{+} \rightarrow \pi^{+} v \bar{v}$, Princeton University PRINCETON/HEP/95-9 Nov. 1995

I-H. Chiang, et al., A new CsI (undoped) Endcap Photon veto system for BNL E787: A study of the decay $K^{+} \rightarrow$ $\pi^{+} v \bar{v}$. Proc. of $V^{\text {th }}$ Intl. Conf. on Calorimetry in High Energy Physics, H. Gordon and D Ruger eds, 103 (1995).

A. Konaka, Status of BNL E787, Proc. 23rd INS Intl. Symposium, Tokyo, Japan, March 1995, S. Sugimoto and O. Hashimoto, Editors, p. 119-127, 1995.

I. Chiang, et al., CsI endcap photon detector for $K^{+} \rightarrow \pi^{+} v \bar{v}$ experiment at BNL, IEEE Trans. Nucl. Sci. vol NS42, no. 4, pp 394-400, August 1995.

S. Adler, et al., Search for the decay $K^{+} \rightarrow \pi^{+} v \bar{v}$, Phys. Rev. Lett. 76, 1421,(1996).

E. W. Blackmore, D. A. Bryman, Y. Kuno, C. Lim, T. Numao, P. Padley, G. Redlinger, R. Soluk, Central tracking chamber with inflated cathode-strip foils, Nucl. Instr. Meth. A404, p.295 (1996).

M. Convery (for the E787 collaboration), First measurement of the structure dependent decay $K^{+} \mu^{+} \gamma \gamma$, Proc. 1996 Annual Mtg. of American Physical Society, Div. of Particles and Fields (DPF '96), Minneapolis, MN., Aug. $10-15,1996$.

S. Adler (for the E787 collaboration), First observation of $K^{+} \rightarrow \pi^{+} \gamma$, Proc. of 1996 Annual Mtg. of American Physical Society, Div. of Particles and Fields (DPF'96), Minneapolis, MN., Aug. 10-15, 1996.

T. Shinkawa, BNL E787 search for $K^{+} \rightarrow \pi^{+} \nu \bar{v}$, Proc. Workshop on K Physics, Orsay, France, May 30-June 4, 1996, L. Iconomidou-Fayard, ed., Editions Frontieres, 389-94 (1997).

S. Kettell (for the E787 collaboration), E787: A search for the rare decay $K^{+} \rightarrow \pi^{+} v \bar{v}$, Proc. 1996 workshop on Heavy Quarks Physics at Fixed Target, Rhinefels Castle, St. Goar, Oct. 1996, L. Köpke, Ed., 397 (1997).

T. Komatsubara, Progress of the rare decay experiment 787 at BNL-AGS, High Energy News (Japan), 16, No. 2, 27 (1997) (in Japanese).

S. Adler, Status report on the search for $K^{+} \rightarrow \pi^{+} v \bar{v}$ and prospects for the search for $K_{\mathrm{L}}^{\circ}$, Proc. of 16th. Intl. Workshop on Weak Interactions and Neutrinos (WIN 97), eds. G. Florillo, V. Palladino, P.Strolin, North-Holland (1998), Nuclear Physics B,Vol. 66, p.466-8 (1998).

D. A. Bryman, et al., 500 MHZ transient digitizers based on GaAs CCDs, Nucl. Instrum. Meth. A396 (1997) 394. 
S. Adler, et al., Evidence for the decay $K^{+} \rightarrow \pi^{+} v \bar{v}$, Phys. Rev. Lett 79, 2204-2207, (1997).

P. Kitching, et al., Observation of the decay $K^{+} \rightarrow \pi^{+} v \bar{v}$, Phys. Rev. Lett. Vol.79, No. 21, 4079-4082, Nov. 24, 1997.

S. Adler, et al., Observation of the decay $K^{+} \rightarrow \pi^{+} \mu^{+} \mu^{-}$, Phys. Rev. Lett., Vol. 79 No. 24, p. 4756-4759, Dec. 15, 1997.

E. W. Blackmore, D. A. Bryman, Y. Kuno, C. Lim, T. Numao, P. Padley, G. Redlinger, R. Soluk, Cenral tracking chamber with inflated cathode-strip foils, Nucl. Instrum. Meth. A 404, 394-404 (1997).

S. Adler, et al., Upper limit on the decay $K^{+} \rightarrow e^{+} v \mu^{+} \mu^{*}$, Phys. Rev. D58, 012003 (1998).

I-H. Chiang, Evidence of $K^{+} \rightarrow \pi^{+} v \vec{v}$ : the BNL E787 1995 result (how did we get here), Proc. Intl. KEK workshop on Kaon, Muon, Neutrino Physics and Future, KEK, Oct. 1997, ed. Y. Kuno, KEK Proc. 97-124, 79 (1998).

'I. Komatsubara, et al., Performance of fine mesh photomultiplyer tubes designed for an undoped CsI endcap photon detector, Nucl. Instrum. Meth. A 404, 315 (1998).

J. A. MacDonald, Future stopped $K^{+} \rightarrow \pi^{+} v \bar{v}$ experiment, Proc. Intl. KEK Workshop on Kaon, Muon, Neutrino Physics and Future, KEK, Oct. 1997, eds. Y. Kuno and T. Shinkawa, KEK Proceedings 97-124, JHF-97-8, 93 (1998).

D. Bryman, Summary, Proc. Intl. KEK Workshop on Kaon, Muon, Neutrino Physics and Future, KEK, Oct. 1997, eds. Y. Kuno and T. Shinkawa, KEK Proceedings 97-124, JHF-97-8, 329 (1998).

L. Littenberg, Can BNL-style studies of $K \rightarrow \pi v \bar{v}$ be pushed at the FEMC?, Proc. of the Workshop on Physics at the First Muon Collider and at the Front End of a Muon Collider, AIP Conf. Proc. 435, Eds. S.H.Geer and R. Raja, pp 299-307 (1998).

G. Guttierez and L. Littenberg, Physics with low energy hadrons. Proc. of the Workshop on Physics at the First Muon Collider and at the Front End of a Muon Collider, AIP Conf. Proc. 435, Eds. S.H.Geer and R. Raja, pp 121138 (1998).

T. Komatsubara, Status of rare kaon decay experiments, Proc. Yukawa Inst. of Theoretical Physics (YITP) worksho on Flavor Physics, Jan. 28-30, 1998, Soryushiron Kenkyu (Kyoto) 98, C29-C37 (1998).

T. Komatsubara, Rare kaon decay experiment, Intl. Workshop on Fermion Masses and CP Violation, Mar 5-6, 1998, Hiroshima, eds. T. Morozumi and T. Mura, Hiroshima U., pp 140-155 (1998).

Y. Kuno and L. Littenberg, Particle physics with kaons, muons and neutrinos, - summary of the JHF K-arena working groups Ia/b, Proc. Intl. Workshop on JHF Science, Mar 1998, eds. J. Chiba, M. Furusaka, H. Miyatake and S. Sawada, KEK Proc. 98-5, JHF 98-2, I-149-I-180 (1998).

L. Littenberg, Rare kaon, muon and pion decay, Proc. $18^{\text {th }}$ Intl. Conf. Physics in Collision, INFN Frascati, June 17-19, 1998, ed. S. Biano, A. Calcaterra, P. deSimonc and F. L. Fabbri, pp 317-334 (1998).

T. Sato, et al., The end cap detector for BNL-E787, Proc. of Workshop on Scintillation Crystals, August, 1997. KEK, Tsukuba, Japan, Proceedings 97-9 (1997).

T. Komatsubara, et al., Performance of fine mesh photomultiplier tubes designed for an undoped CsI endcap photon detector, Nucl. Instrum. Meth. A404, 315 (1998).

S. Kettell, Evidence for $K^{+} \rightarrow \pi^{+} v \bar{v}$ and search for T-violation in Kmu3, Proc. Workshop on Heavy Quarks at Fixed Target; FNAL, Oct. 12, 1998, ed. H. Cheung and J. Butler, pp 421-430 (1998). 
M. V. Diwan, Observation of the decay $K^{+} \rightarrow \pi^{+} v \bar{v}$, Proc. APS meeting of Div. Of Particles and Fields (DPF99), Los Angeles, Calif. Jan. 5-9, 1999, e-Print archive hep-ex/9903026 (1999).

T. Komatsubara, Status of the study of the rare decay $K^{+} \rightarrow \pi^{+} v \bar{v}$ at BNL, proc. $17^{\text {th }}$ Intl. Workshop on Weak Interactions and Neutrinos (WIN99), Eds. C. A. Dominguez and R. D. Viollier, World Scientific, ISBN 981-024082-1, pp535-539 (2000).

S. Adler, et al, Further search for the decay $K^{+} \rightarrow \pi+v \bar{v}$, Phy. Rev. Lett. 84, pp.3768-3770 (2000).

S. Adler, et al., Measurement of structure dependent $K_{r}^{+} \rightarrow \mu^{+} v \gamma$, Phys. Rev. Lett. 85, pp.2256-2259 (2000).

M. V. Diwan, Observation of the decay $K^{+} \rightarrow \pi+v \bar{v}$, proc. Am. Phys. Soc. Mtg. of the Division of Particles and Fields (DPF 99), Los Angeles, Calif., Jan. 5-9, 1999. e-Print Archive: hep-ex/9903026.

B. F. Ng, the search for the rare decay of $K^{+} \rightarrow \pi+\pi^{0} v \bar{v}$, SUSB Ph.D. thesis, May 2000.

J. Doombos, P. Pile, F. Meot, M. Aoki, E.W. Blackmore, I-H. Chiang, C.J. Kost, K.K. Li, J.A. Macdonald, T. Nakano, Optics design and performance of LESB3, a two-stage separated $800 \mathrm{MeV} / \mathrm{c}$ kano beamline, Nucl. Inst. \& Meth. A444, pp.546 (2000).

S. Adler, et al., Measurement of direct photon emission in $K^{+} \rightarrow \pi^{+} \pi^{0} \gamma$ decay, Phys. Rev. Lett. in press (2000).

S. Kettell, Measurement of $B\left(K^{+} \rightarrow \pi^{+} v \bar{v}\right)$, to be published in proc. of $7^{\text {th }}$ Conf. on Intersections Between Particle and Nuclear Physics (IPANP 2000), Quebec City, Quebec, Canada, May 22-28,. 2000, e-Print Archive: hep-ex/0008077.

T. Komatsubara, Recent results from the BNL E787 experiment, to be published in Proc. of the $30^{\text {th }}$ Intl. Conf. on High Energy Physics ICHEP2000, Osaka, Japan, July 27-Aug. 2, 2000. e-Print Archive: hep-ex/0009047.

S. Adler, Search for the decay $K^{+} \rightarrow \pi^{+} \pi^{o} v \bar{v}$, submitted to Phys. Rev. D, 63, 032004 (2001).

S. Adler et al, Measurement of structure-dependent $K^{+} \rightarrow \mu^{+} v_{\mu} \gamma$ decay, Phys. Rev. Let. 85, 11 pp. 2256-2259, September 11, 2000.

$788 \quad$ J.J. Szymanski, Weak decay of $\Lambda^{0}$ hypernuclei, Proc. of the 2nd Conf. on the Intersections between Particle and Nuclear Physics, Lake Louise, Alberta, Canada, May 24, 1986.

M. Athanas, et. al., Weak decay of light hypernuclei, invited talk by R.A. Schumacher at the JSPS-NSF Joint Seminar on "Hyperon Nucleon Interactions", Maui, HI, October, 1993.

789 J. Sculli, et al., Limits on $\xi(2.2)$ formation in $\mathrm{p} \overrightarrow{\mathrm{p}} \rightarrow K^{+} K^{-}$; Phys. Rev. Lett. 58, 1715, (1987).

790 L.P. Remsberg, et al., 7th High Energy Heavy Ion Study, Stopping power measurements with $17 \mathrm{GeV} / \mathrm{c}$ protons at the AGS or inclusive proton spectra from proton-nucleus interactions at $17 \mathrm{GeV} / \mathrm{c}, \mathrm{R}$. Bopck, H.H. Gutbrod, R. Stock (eds.), Gesellschaft für Schwerionenforschung mbH., Darmstadt, FRG, March 1985, p. 439-450.

791 R.D. Cousins, et al., Search for the decays and $\mathrm{K}_{\mathrm{L}}^{\circ} \rightarrow \mu e$ and $\mathrm{K}_{\mathrm{L}}^{0} \rightarrow e^{+} e^{-}$; Phys. Rev. D38 (Rapid Communications) 2914-2917 (1988).

C. Mathiazhagan, et al., New experimental limits on $\mathrm{K}_{\mathrm{L}}^{0} \rightarrow$ ue and $\mathrm{K}_{\mathrm{L}}^{0} \rightarrow e^{+} e^{-}$branching ratios, Phys. Rev. Lett. $632128-2184$ (1989). 
C. Mathiazhagen, et al., Measurement of the branching ratio for the decay $\mathrm{K}_{\mathrm{L}}^{\circ} \rightarrow \mu^{+} \mu^{-*}$, Phys. Rev. Lett. 63 2185-2188 (1989).

A. Heinson, et al., Higher statistic measurement of the branching ratio for the decay $\mathrm{K}_{\mathrm{L}}^{0} \rightarrow \mu^{+} \mu^{-*}$, Phys. Rev. D (Rapid Communications), D44, 1-5 (1991).

$\mathrm{K}$. Arisaka, et al., Improved upper limit on the branching ratio $B\left(\mathrm{~K}_{\mathrm{L}}^{\mathrm{o}} \rightarrow \mu^{ \pm} e\right)$, Phys. Rev. Lett. 70,1044 (1993).

$\mathrm{K}$. Arisaka, et al., Improved sensitivity in a search for the rare decay $\mathrm{K}_{\mathrm{L}}^{\circ} \rightarrow e^{+} e^{-}$, Phys. Rev. Letts., 71, 3910 (1993).

793 Y. D. He, P. B. Price, and W. T. Williams, Interactions of projectile fragments at $14.5 \mathrm{~A}$ GeV: A search for anomalons, Phys. Lett. B 252, 331-335 (1990).

P. B. Price and Y. D. He, Behavior of nuclear projectile fragments produced in collisions of $14.5 \mathrm{~A} \mathrm{GeV}{ }^{28}$ Si with $P b$ and $C u$ targets, Phys. Rev. C 43, 835-848 (1991).

Y. D. He and P. B. Price, Search for fractional charge states in high-energy heavy fragments produced in collisions of 14.5 $\mathrm{A} \mathrm{GeV}^{28}$ Si with Pb and $\mathrm{Cu}$ target, Phys. Rev. C 44, 1672-1674 (1991).

Y. D. He and P. B. Price, A search for fractional charge states in high-energy heavy fragments produced in collisions of $14.5 \mathrm{~A} \mathrm{GeV}^{28}$ Si with $\mathrm{Pb}$ and $\mathrm{Cu}$ target, Proc. of $22^{\text {nd }}$ Intl. Cosmic Ray Conf., Dublin, 4, 734-737 (1991).

Y. D. He and P. B. Price, Sensitivity study of CR-39 plastic track detectors, Nucl. Tracks Radia. Meas. 20, 491-494 (1992).

794 D.C. Peaslee, et al., Large $\mathrm{P}_{\perp}^{2}$ spin effects in $p p \rightarrow p p$, Phys. Rev. Lett. 51,2359 (1983).

P.R. Cameron, et al., Measurement of the analyzing power for $p p \rightarrow$ pp at $\mathrm{P}_{\perp}^{2}=6.5(\mathrm{GeV} / \mathrm{c})^{2}$, Phys. Rev. D32, 3070(1985).

D.G. Crabb. et al., High precision measurement of the analyzing power in large $\mathrm{P}_{\perp}^{2}$ spin-polarized $24 \mathrm{GeV} / \mathrm{c}$ proton-proton elastic scattering, Phys. Rev. Lett. 65, 3241 (1990).

D.G. Crabb, et al., Observation of a 96\% proton polarization in irradiated ammonia, Phys. Rev. Lett. 64, 2627 (1990).

795 B. Bassalleck, $N \square N$ bound states, Proc. of 1986 INS Intl. Symposium on Hypernuclear Physics, Tokyo, p. 385.

796 Y.J. Uemura, et al., Muon spin relation in $\mathrm{CeCu}^{2} \mathrm{Si}^{2}$ and muon knight shift in various heavy-fermion systems, 4th Int. Conf. on Muon Spin Rotation, Relaxation and Resonance, Uppsala, Sweden, June 1, 1986.

W.J. Kossler, et al. Magnetic field penetration depth of $\mathrm{La}_{1.85} \mathrm{Sr}_{0.15} \mathrm{CuO}_{4}$ measured by muon spin relaxation, Phys. Rev. B, 35, 7133 (1987).

W.J. Kossler, et al., Muon spin relaxation on high- $T_{c}$ superconductors, Novel Super-conductivity, 757, Stuart A. Wolf and Vladimir Z. Kresin, Editors (Plenum Publishing Corp., 1987).

Y.J. Uemura, et al., Antiferromagnetism of $\mathrm{La}_{2} \mathrm{CuO}_{4-y}$ studied by muon-spin rotation, Phys. Rev. Lett. 59, 1045 (1987).

Y.J. Uemura, et al., Systematic variation of magnetic-field penetration depth in high- $T_{c}$ superconductors studied by muon-spin relaxation, Phys. Rev. B, 38 (1988). 
J.H. Brewer, et al., Antiferromagnetism and superconductivity in oxygen-deficient $\mathrm{YBa}_{2} \mathrm{Cu}_{3} \mathrm{O}_{x}$, Phys. Rev. Lett., 60,1073 (1988).

Y.J. Uemura, et al., Comparison between muon spin rotation and neutron scattering studies on the 3-dimensional magnetic ordering of $\mathrm{La}_{2} \mathrm{CuO}_{4-y, y}$ Physica C, 769-70 (1988).

Y.J. Uemura, et al., Universal correlations between $T_{c}$ and $n_{s} / m^{*}$ (carrier density over effective mass) in high- $T_{c}$ cuprate superconductors, Phys. Rev. Lett. 62, 2317 (1989).

W.J. Kossler, et al., Coexisting static magnetic order and superconductivity in $\mathrm{CeCu}_{2.1} \mathrm{Si}_{2}$ found by muon spin relaxation, Phys. Rev. B., 39, 4726 (1989).

798 H.S. Plendl, Excitation of hypernuclear states through the $\left(\pi^{+}, K^{+}\right)$reaction, Proc. of the 4 th Intl. Conf. on Nuclear Reaction Mechanisms, Varenna, June 10-15, 1985. Ed. E. Gadioli. R.E. Chrien, Nucl. Phys. A478 (1988) $705 c$.

P.H. Pile, J. Phys. Soc. Japan. 58 (1989) Suppl. p. 394.

P.H. Pile, A study of heavy hypernuclei using the $\left(\pi^{+}, \mathrm{K}^{+}\right)$reaction, IL Nuovo Cimento, Vol. 102, No. 2, p. 413 (1989).

P. H. Pile, $\pi^{+}{ }^{+} K^{+}$experimental difficulties, BNL-45637-mc (microfiche), Workshop on $(\pi, \mathrm{K})$ Physics, Los Alamos, NM, Oct.10, 1990. Pub.in LAMPF K workshop 1990, p.77-85 (QCD161:L2:1991).

P.H. Pile, et al., Study of hypernuclei by associated production, Phys. Rev. Letts. 66, 2585-1258 (1991).

801 Gordon L. Shaw, et al., Search for free quarks produced by 14.5 GeV/nucleon oxygen ions, Phys. Rev. D, 36,3533 1987.

802 T. Abbott, et al., Measurement of energy emission from $O+A$ and $p+A$ collisions at $14.5 \mathrm{GeV} / \mathrm{c}$ per nucleon with a lead-glass array, Phys. Lett. 197, 285-90 (1987).

T. Abbott, et al., First encounter with a $14.5 \mathrm{GeV} /{ }^{16} \mathrm{O}$ beam. Proc. XI Intl. Conf. on Particle and Nuclei (PANIC 87), Kyoto, Japan, April 20-24, 1987 (Abstract Book Part II), edited by S. Homma et al., p. 448-9 (1987).

D.E. Alburger, et al., CsI(Ti)- ${ }^{241}$ Am calibration source for Pb-glass detectors, Nucl. Instr. and Meth. A254, 88 (1987).

D.E. Alburger, Removal of phototubes from Pb-glass detectors, Rev. Sci. Instrum. 58, 143 (1987).

D.E. Alburger and M.J. Tannenbaum, Removal of phototubes from Pb-glass detectors II, Rev. Sci. Instrum. 58, 1761 (1987).

M.J. LeVine, W.A. Watson III, H. von der Schmitt, and S. Kaufman, Distributed data acquisition for BNL 802 I: the front end, Fifth Conf. on Real-Time Computer Applications in Nuclear, Particle and Plasma Physics, San Francisco, CA, May 11-15, 1987, IEEE Trans. Nucl. Sci. NS-34, 830 (1987).

R.A. Scheetz and M.J. LeVine, An intelligent VME-based camac crate controller, Fifth Conf. on Real-Time Computer Applications in Nuclear, Particle, and Plasma Physics, San Francisco, CA, May 11-15, 1987, IEEE Trans. Nucl. Sci. NS-34, 1033 (1987).

R.A. Scheetz and M.J. LeVine, A VME-VMX interface to fastbus via the LeCroy 1821 segment manager, Fifth Conf. on Real-Time Computer Applications in Nuclear, Particle, and Plasma Physics, San Francisco, CA, May 11-15, 1987, IEEE Trans. Nucl. Sci. NS-34, 1036 (1987). 
B. Wadsworth, et al., The trigger supervisor: managing triggering conditions in a high energy physics experiment, Fifth Conf. on Real-Time Computer Applications in Nuclear, Particle, and Plasma Physics, San Francisco, CA, May 11-15, 1987, IEEE Trans. Nucl. Sci. NS-34, 980 (1987).

T. Abbott, et al., Measurement of energy and charged particle emission in the central rapidity region from $O+A$ and $p+A$ collisions at $14.5 \mathrm{GeV} / \mathrm{c}$ per nucleon and preliminary results from $\mathrm{Si}+\mathrm{A}$ collisions. Proc. 6th Intern. Conf. on Ultra-Relativistic Nucleus-Nucleus Collisions, August 24-28, 1987, Schloss- Nordkirchen, W. Germany. Z. Phys. C $\underline{38}, 35-43$ (1988).

T. Abbott, et al., Preliminary spectrometer results from E802, Proc. 6th Int. Conf. on Ultra-Relativistic Nucleus-Nucleus Collisions (Quark Matter 1987), Z. Phys. C 38, 135-9 (1988).

T. Abbott, et al., Measurement of energy and charged particle emission in the central rapidity region from $O+A$ and $p+A$ collisions at $14.5 \mathrm{GeV} / \mathrm{c}$ per nucleon and preliminary results from $S i+A$ collisions, Proc. 6th Int. Conf., on Ultra-Relativistic Nucleus-Nucleus Collisions (Quark Matter 1988), Z. Phys. C.

T. Abbott, et al., Proton production from $\mathrm{Si}+\mathrm{Au}$ collisions at $14.5 \mathrm{~A} \cdot \mathrm{GeV}$, Proc. 7 th Int. Conf. on Ultra-Relativistic Nucleus-Nucleus Collisions (Quark Matter 1988), Z. Phys. C.

T. Abbott, et al., Particle spectra near mid-rapidity studied with a Cherenkov-complex system in $14.5 \mathrm{~A} \mathrm{GeV} \mathrm{Si+}$ Au collisions, Proc. 7th Int. Conf. on Ultra-Relativistic Nucleus-Nucleus Collisions (Quark Matter 1988), Z. Phys. C.

T. Abbott, et al., Preliminary results from Brookhaven experiment 802 with $14.5 \mathrm{GeV} /{ }^{28} \mathrm{Si}$. Proc. IX Autumn School on the Physics of Quark-Gluon Plasma. December 9-12, 1987, Lisbon, Portugal. J. Dias de Deus and S. Costa Ramos, editors, World Scientific Publishing Co., Pte. Ltd., New Jersey, p. 3-14 (1988).

T. Abbott, et al., Preliminary spectrometer results from E802. Proc. 6th Intern. Conf. on Ultra-Relativistic Nucleus-Nucleus Collisions, Aug. 24-28, 1987, Schloss-Nordkirchen, Germany. Z.Phys.C 38, 135-9 (1988).

T. Abbott, et al., Projectile energy degradation at $14.5 \mathrm{GeV} / u$. Proc. Third Int. Conf. on Nucleus-Nucleus Collisions, June 6-11 1988, Saint-Malo, France.

T. Abbott, et al. Spectrometer results from BNL E802. Proc. 3rd Conf. on the Intersections Between Particle and Nuclear Physics, May 14-19, 1988, Rockport, ME. G.M. Bunce, Editor, AIP Conf. Proc. 176, 1988, p. 1060-7.

M.J. Tannenbaum, et al, Measurement of energy flow from oxygen, silicon and proton inter-actions with nuclei at the BNL Tandem-AGS. Proc. XXIII Recontre Moriond, Current Issues in Hadron Physics, Les Arcs, Savoie, France, March 1988.

P. Vincent, et al., The E802 aerogel Cerenkov detector, Nucl. Inst. and Meth. A272, 660 (1988).

T. Abbott, et al., Proton production form Si+Au collisions at $14.5 \mathrm{~A} \mathrm{GeV}$. Proc. 7th Intern. Conf. on UltraRelativistic Nucleus-Nucleus Collisions, Sept.26-30, 1988, Lenox, MA. Nucl. Phys. A498, 409c-13c (1989).

T. Abbott, et al., Particle spectra near mid-rapidity studies with a Cherenkov-complex system in $14.5 \mathrm{~A} \mathrm{GeV} \mathrm{Si+}$ Au collisions. Proc. 7th Intern. Conf. on Ultra-Relativistic Nucleus-Nucleus Collisions, September 26-30, 1988. Lenox, MA. Nucl. Phys. A498, 415c-9c (1989).

T. Abbott, et al., Results from the BNL E802 spectrometer for $14.5 \mathrm{GeV/c}$ per nucleon Si beams. Proc. 7th Intern. Conf. on Ultra-Relativistic Nucleus-Nucleus Collisions, Sept. 26-30, 1988, Lenox, M.A.Nucl. Phys. A498, $67 \mathrm{c}-78 \mathrm{c}(1989)$.

T. Abbott, et al., Results for strange production from BNL E802, Proc. Hadronic Matter in Collision, Oct. 6-12, 1988, Tucson, AZ. P.Carruthers and J.Rafelski, Editors, World Scientific, p. 607-16 (1989). 
T. Abbott, et al., BNL high energy heavy ion experiments, Proc. 5th French-Japanese Symposium on Nuclear Physics, Sept. 26-30, 1989, Dogashima, Japan, p. 33-41 (1989).

T. Abbott, et al., Survey of results from Brookhaven experiment 802 at the AGS. Proc. Symp. on Nuclear Dynamics and Nuclear Disassembly, American Chemical Society, April 10-14, 1989, Dallas, TX. J.B. Natowitz, Ed., World Scientific, p. 507-16 (1989).

D. Alburger, et al., Response of a thin avalanche detector to simultaneous minimum ionizing particles, Nucl. Instr. and Meth. A276, 127 (1989).

D. Beavis, et al., A calorimeter for relativistic heavy-ion experiments, Nucl. Instr. Meth. A281, 367-72 (1989).

J.B. Cumming, et al., More on ZCAL's response: position dependence, position determination, and collimation effects. BNL Informal Report, BNL-43537 (1989). R. Debbe, J. Fischer, D. Lissauer, T. Ludlam, D. Makowiecki, V. Radeka, S. Rescia, G.C. Smith, D. Stephani, D. Yu. M.W.P.C. with highly segmented cathode pad readout. Proc. 1989 Wire Chamber Conf., February 13-17, 1989, Vienna, Austria. Nucl. Instr. Meth. A283, 772-7 (1989).

H. Wegner, et al., (E-802 Collaboration), $p+A$ and comparison to ${ }^{28} \mathrm{Si}+\mathrm{A}$ measurements with the E-802 relativistic heavy ion spectrometer, Proc.1989 Intl. Nuclear Physics Conf., August 20-26, 1989, Sao Paulo, Brazil.

T. Abbott, et al., Kaon and pion production in central Si+Au collisions at $14.6 \mathrm{~A} \mathrm{GeV/c}$, Phys. Rev. Letts. 64, $847-50$ (1990).

T. Abbott, et al., A single arm spectrometer detector system for high-energy heavy ion experiments, Nucl. Instr. Meth. A 290, 41-60 (1990).

R. Debbe, et al., A study of wire chambers with highly segmented cathode pad readout for high multiplicity, charged particle detection. IEEE Nuc. Sci. Symposium, Jan. 17-19, 1990, San Fran., CA. IEEE Trans. Nucl. Sci. 37, 88-94 (1990)

O. Hansen, Strangeness production in Si+Au interactions at $14.6 \mathrm{GeV} / \mathrm{c}$ per nucleon. Proc. Intl. Advanced Courses on the Nuclear Equation of State, May 21-June 3, 1989, Pensicola, Spain, "The Nuclear Equation of State, Part B, edited by W. Greiner and H. Stöcker, p. 97-102, Plenum Press, New York (1990).

O. Hansen, Are hadron spectra thermal in $14.6 \mathrm{~A}$ GeV/c nucleus-nucleus collisions? Proc. 6th Nordic Meeting on Nuclear Physics, August 10-15, 1989, Utgarden, Kopervik, Norway. Physica Scripta T32, 143-6 (1990).

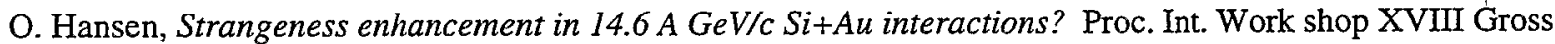
Properties of Nuclei and Nuclear Excitations, January 15-20, 1990. Hirschegg, Austria, p. 63-70 (1990).

O. Hansen, Editor. Proc. Workshop on Heavy Ion Physics at the AGS (HIPAGS), March 5-7, 1990, Brookhaven National Laboratory, Upton, NY. BNL-44911 (1990).

Y. Miakc, ct al., (E-802 Collaboration). Spectra and strangeness production. Proc. Workshop on Heavy Ion Physics at the AGS (HIPAGS), March 5-7, 1990, Brookhaven National Laboratory, Upton, NY. BNL-44911, p. 240-8 (1990).

M.J. Tannenbaum, et al., (E-802 Collaboration). E $E_{T}$ distributions, "nuclear stopping," and correlations among measurements from the 4 detector systems in AGS E802. Proc. Workshop on Heavy Ion Physics at the AGS (HIPAGS), March 5-7, 1990, Brookhaven National Laboratory, Upton, NY BNL-44911, p. 44-69 (1990).

F. Videbaek, et al., (E-802 Collaboration). Charged particle distributions in heavy ion collisions at $14.6 \mathrm{GeV} \mathrm{A} / \mathrm{c}$. Proc. Workshop on Heavy Ion Physics at the AGS (HIPAGS), March 5-7, 1990, Brookhaven National Laboratory, Upton, NY. BNL-44911, p. 38-43 (1990). 
J. Costales, et al., (E-802 Collaboration). E802: baryons and anti-baryons. Proc. Workshop on Heavy Ion Physics at the AGS (HIPAGS), March 5-7, 1990, Brookhaven National Laboratory, Upton, NY. BNL-44911, p. 249-60 (1990).

R.J. Morse, et al., (E-802 Collaboration). E802 HBT results. Proc. Workshop on Heavy Ion Physics at the AGS (HIPAGS), March 5-7, 1990, Brookhaven National Laboratory, Upton, NY. BNL-44911, p. 402-10 (1990).

M.J. LeVine, New challengers for HEP computing: RHIC and CEBAF. Proc. Computing in High Energy Physics-1990, Santa Fe, NM. AIP Conf. Proc. 209, J. Lillberg and M. Oothoudt, Editors, p. 31-43 (1990).

T. Abbott, et al., Antiproton production in 14.6 A GeV́V/c Si + A Collisions, Phys. Lett. B271, 447-52 (1991).

T. Abbott, et al., Forward and Transverse Energies in Relativistic Heavy Ion Collisions at $14.6 \mathrm{GeV} / \mathrm{c}$ per Nucleon, Phys. Rev. C 44, $1611-9$ (1991).

T. Abbott, et al., Comparison of $p+A$ and Si+Au Collisions at 14.6 GeV/c. Phys. Rev. Letts. 66, 1567-70 (1991).

M.A. Bloomer, et al., (E-802 Collaboration). Love, W. et al (E-810 Collaboration), and L. Waters (E-814 Collaboration). New results from AGS heavy-ion experiments. Nucl. Phys. A527, 595c-600C (1991).

J.B. Costales, et al., (E-802 Collaboration). Antiproton production in $14.6 \mathrm{~A} \mathrm{GeV/c} \mathrm{Si}+\mathrm{A}$ collisions. Proc. Quark Matter 1990, May 7-11, 1990, Menton, Francc. Nucl. Phys. A525, 455c-8c (1991).

J.B. Costales, et al., (E-802 Collaboration). Target rapidity proton distributions for Si+A collisions at the AGS. Proc. Quark Matter 1991, November 11-15, 1991, Gatlinburg. TN.

O. Hansen, On the quark-gluon plasma and strangeness enhancement. Comments Nucl. Part. Phys., Vol. 20, Nos. $1 \& 2$, p. 1-14 (1991).

O. Hansen, Nucleus-nucleus collisions at very high energies. Proc. Mikolajki Summer School on Nuclear Physics, August 25-September 5, 1990, Mikolajki, Poland in "Nuclear and Atomic Physics with the Accelerators of the Nineties," Editors, A. Sujkowski and G. Szeflinska, IOP Publishing, Ltd, p. 1-18 (1991).

Y. Miake, et al., (E-802 Collaboration). Particle production in Si $+A$ and $p+A$ collisions at $14.6 \mathrm{~A} \mathrm{GeV/c.} \mathrm{Proc.}$ Quark Matter 1990, May 7-11, 1990, Menton, France. Nucl. Phys. A525, 23 1c-6c (1991).

R.J. Morse, et al., (E-802 Collaboration). Bose-Einstein correlations in $14.6 \mathrm{~A} \mathrm{GeV/c}{ }^{28} \mathrm{Si}+\mathrm{A}$ collisions. Proc. Quark Matter 1990, May 7-11, 1990, Menton France. Nucl. Phys. A525, 531c-5c (1991).

B. Moskowitz, et al., (E-802 Collaboration). A review of recent E802 results. Proc. Seventh Winter Workshop on Nuclear Dynamics, January 26-February 2, 1991, Key West, FL.

M.J. Tannenbaum, et al., (E-802 Collaboration). Transverse energy and multiplicity distributions of $p+p$ and $A+A$ interactions. Proc. Quark Matter 1990, May 7-100, 1990, Menton, France. Nucl. Phys. A525, 681c-4c (1991).

F. Videbaek, et al., (E-802 Collaboration). Particle production and spectra in E-802 at BNL-AGS. Proc. Int. Symp. of High Energy Nuclear Collisions and Quark Gluon Plasma, June 6-8, 1991, Kyoto, Japan.

W.A. Zajc, et al., (E-802 Collaboration). Recent results from E802 and E859. Proc. Quark Matter 1991, November 11-15, 1992, Gatlinburg, TN..

T. Abbott, et al., Bose-Einstein Correlations in Si + Al and $S i+A u$ Collisions at $14.6 \mathrm{~A} \mathrm{GeV/c.} \mathrm{Phys.} \mathrm{Rev.} \mathrm{Letts.}$ 69, 1030-3 (1992). 
T. Abbott, et al., Measurement of particle production in proton induced reactions at $14.6 \mathrm{GeV} / \mathrm{c}$. Phys. Rev. D 45, 3906-20 (1992).

T. Abbott, et al., Centrality dependence of $\mathrm{K}^{+}$and $\pi^{+}$multiplicities from Si+A collisions at $14.6 \mathrm{~A} \mathrm{GeV/c}$. Phys. Lett. B 291, 341-6 (1992).

T. Abbott, et al., Global transverse energy distributions in relativistic nuclear collisions at 14.6 A GeV/c. Phys. Rev. C 45, 2933-51 (1992).

R. Moskowitz, O. Hansen and F. Videbaek. An "in acceptance" comparison of $S i+$ Au data and RQMD predictions. Proc. Gross Properties of Nuclei and Nuclear Excitations Intl. Workshop XX, January 20-25, 1992, Hirschegg, Kleinwalsertal, Austria.

T. Abbott, et al., Multiplicity distributions from central collisions of ${ }^{16} \mathrm{O}+\mathrm{Cu}$ at $14.6 \mathrm{~A} \mathrm{GeV/C}$ and inter-mittency. Accepted by Phys. Rev. C, September 7, 1995.

Y. Akiba, et al., Two-particle rapidity correlations from the Bose-Einstein effect in central ${ }^{28}$ Si+Au collisions at14.6A Ge/v/c and intermittency, Phy. Rev. C, Vol. 56, 3, pp.1544 (1997).

805 S. De Panfilis, et al., First results from the galactic axion search, Proc. of the 13th Texas Symposium on Relativistic Astrophysics, M. Ulmer, etc., (World Scientific, 1987) p. 265.

S. DePanfilis, et al., Limits on the abundance and coupling of cosmic axions at $4.5<m_{a}<5.0 \mu \mathrm{eV}$, Phys. Rev. Letts. 59, 839 (1987).

S. De Panfilis, et al., Limits on the abundance and coupling of cosmic axions. Neutrino Masses and Neutrino Astrophysics, Proc. of the IV Telemark Conf., V. Barger, F. Halzen, M. Marshak, and K. Olive, ed. (World Scientific, 1987).

S. De Panfilis and J. Rogers, $L$ and $S$ band low-noise cryogenic GaAsFET amplifiers. IEEE Trans. Microwave Theory and Techniques (March 1988).

S. De Panfilis, et al., An update of results from an experiment to search for galactic axions. Proc. of II Workshop on Low Temperature Devices for Detection of Low Energy Neutrinos and Dark Matter, L.A.P.P. (Annecy, France), May, 1988.

B.E. Moskowitz, Cosmic axion searches. Proc. of the Workshop on Non-Accelerator Particle Physics, A.C. Melissinos and B.E. Moskowitz, eds., NIM A264, 98 (1988).

B.E. Moskowitz and J. Rogers, Analysis of a microwave cavity detector coupled to a noisy amplifier. Nuclear Instruments and Methods A264, 445 (1988).

B.E. Moskowitz, et al., Improved limits from the galactic axion search, Proc. of the II ESO/CERN Symposium on Astronomy, Cosmology, and Fundamental Physics, (Bologna, Italy) May, 1988, G. Giacomclli, cd.

J.T. Rogers, et al., Anomalous r.f magneto resistance in copper at $4^{\circ} \mathrm{K}$, Applied Physics Letters 52, 2266 (1988).

A.C. Melissinos, et al., Search for cosmic axions, Eighth Workshop on Grand Unification, K.C. Wali, Ed., World Scientific, 1988.

W. Wuensch, et al., Search for cosmic axions, Proc. of XXIII Rencontre de Moriond on Dark Matter (Les Arcs, France) 1988. J. Tran Than Van, ed.

W.U. Wuensch, et al., Results of a laboratory search for cosmic axions and other weakly coupled light particles," Phys. Rev. D40, 3153 (1989). 
J.T. Rogers, et al., Experience with the Rochester-Brookhaven-Fermilab axion detector, Proc. of the Workshop on Cosmic Axions (BNL 1989), C. Jones \& A.C. Melissinos, eds.,World Scientific, p.39 (1990).

C. Brechtmann, W. Heinrich, Nucl. Instr. Meth. B, 29 (1988).

A. Hoffmann, C. Brechtmann, W. Heinrich, E.V. Benton, Search for projectile fragments with fractional charge in relativistic heavy ion collisions. Phys. Letts. B, Vol. 200, No. 4, 21 Jan. 1988).

C. Brechtmann, W. Heinrich, and E.V. Benton, Fragmentation cross- sections of ${ }^{28}$ Si at $14.5 \mathrm{GeV}$ Nucl, Phys. Rev. C 39, (1989).

W. Heinrich, C. Brechtmann, Electromagnetic dissociation in ultra-relativistic heavy ion reactions, Mod. Phys. Lett. A 4, 1879-1882 (1989).

308 L.M. Barbier, et al., Central collisions of 14.6,60, and $200 \mathrm{GeV} /$ nucleon ${ }^{16} \mathrm{O}$ nuclei in nuclear emulsion, Phys. Rev. Lett. Vol. 60, No. 5, 405 (1988).

L.M. Barbier, et al., Nucleus-nucleus interactions at 14.6, 60 and 200 GeV/nucleon, Nuclear Physics A498, $535 c-540 c(1989)$.

$\mathrm{H}$. von Gersdorff, et al., Single-particle and multi-particle analysis of nucleus-nucleus collisions at 14.6, 60 and 200 GeV/nucleon, Phys. Rev. C, 39, No. 4, 1385 (April 1989).

R. Holynski, et al., Evidence of intermittent patterns of fluctuations in particle production in high energy interactions in nuclear emulsion, Phys. Rev. Lett., Vol. 62, 733 (1989).

R. Holynski, et al., One- and two-dimensional analysis of the factorial moments in $200 \mathrm{GeV} / \mathrm{nucleon} \mathrm{p},{ }^{16} \mathrm{O}$ and ${ }^{32} \mathrm{~S}$ interactions with Ag and Br nuclei, Phys. Rev. C, Vol. 40, No. 6 (1989).

J.F. Amundson, Intermittency and relativistic heavy-ion Monte Carlo simulators, Phys. Rev. C 41, 1292 (1990).

C. .J. Waddington, et al., Energy dependence of fragmentation of oxygen nuclei up to $200 \mathrm{GeV} /$ nucleon. $21^{\text {st }}$ Intl. Cosmic Ray Conf., University of Adelaide, Australia, 8, 87 (1990).

S.J. Lindenbaum, et al., Search for QGP signals at AGS with a TPC spectrometer, and comparison of our event generator predictions for plasma model and cascade interactions. Proc. of the Third Conf. on the Intersections Between Particle and Nuclear Physics, May 14-19, 1988, Rockport, Maine. AIP Conf. Proc. 176, p. 778-786 (1988).

Kenneth J. Foley, The use of TPC's as target region detectors in the MPS. Proc. of the 1988 BNL Workshop on Glueballs, Hybrids and Exotic Hadrons, August 29-September 1, 1988, Brookhaven Natl. Laboratory, AIP Conf. Proc. 185, Suh-Urk Chung, Editor, p. 643-645.

W.A. Love (The E810 Collaboration), Some TPC measurements in an oxygen beam at the AGS. Proc. of the VII Intl. Conf. on Ultra-Relativistic Nucleus- Nucleus Collisions (Quark Matter 88) Lenox, Massachusetts, Sept. 2630, 1988. G.A. Baym, P. Braun-Munzinger, S. Nagamiya, Eds., Nucl. Phys. A498, 523c-528c (1989).

A. Etkin, et al., A TPC for large solid angle relativistic ion experiments. Proc. of the 1988 IEEE Nuclear Science Symposium, Orlando, Florida, November 9-11, 1988, IEEE Transactions on Nuclear Science 36, p. 58-62 (1989).

A. Etkin, et al., Modular TPC's for relativistic heavy ion experiments, Proc. of the Wire Chamber Conf., Vienna, Austria, Feb.13-17, 1989, Nuclear Instruments and Methods in Physics Research A283, 557-566 (1989).

S.J. Lindenbaum, Search for a QGP with a TPC spectrometer, and QGP signals predicted by new event generator, Proc. of the Hadronic Matter in Collision Conf., Tucson, AZ, Oct. 6-12, 1988, P. Carruthers and J. Rafelski, Editors, p. 673-692 (World Scientific, 1989). 
K.J. Foley, et al., Studies of multiparticle production in heary ion collisions using a time projection chamber, Proc. of the Europhysics Conf. on High Energy Physics, 6-13 Sept. 1989, Madrid, Spain. Nucl. B (Proc. Suppl.) $16,405-406$ (1990).

Alfred C. Saulys (for the E810 Collaboration: B.E. Bonner, J.A. Buchanan, C.S. Chan, C.N. Chiou, J.M. Clement, M.D. Corcoran, A. Etkin, K.J. Foley, R.W. Hackenburg, T.J. Hallman, M.A. Kramer, J.W. Kruk, S.J.

Lindenbaum, R.S. Longacre, W.A. Love, L. Madansky, H.E. Miettinen, T.W. Morris, G.S. Mutchler, M. Nessi, F. Nessi-Tedaldi, E.D. Platner, J.B. Roberts and A.C. Saulys). $V^{\circ}$ production with $14.5 \mathrm{GeV} / \mathrm{c}$ silicon beams. Proc. of the Workshop on Heavy Ion Physics at the AGS, Brook-haven National Laboratory, March 5-7, 1990, Editor: Ole Hansen, p. 209-226 (BNL, 1990).

William A. Love (for the E810 Collaboration: B.E. Bonner, J.A. Buchanan, C.S. Chan, C.N. Chiou, J.M. Clement, M.D. Corcoran, A. Etkin, K.J. Foley, R.W. Hackenburg, T.J. Hallman, M.A. Kramer, J.W. Kruk, S.J.

Lindenbaum, R.S. Longacre, W.A. Love, L. Madansky, H.E. Miettinen, T.W. Morris, G.S. Mutchler, M. Nessi, F. Nessi-Tedaldi, E.D. Platner, J.B. Roberts, and A.C. Saulys). AGS silicon gold collisions measured in the E810 TPC. Proc. of the Workshop on Heavy Ion Physics at AGS, Brookhaven National Laboratory, March 5-7, 1990, Ole Hansen, Editor, p. 27-37 (BNL, 1990).

E.D. Platner (for the E810 Collaboration: A. Etkin, K.J. Foley, R.W. Hackenburg, R.S. Longacre, W.A.Love, T.W. Morris, E.D. Platner, A.C. Saulys, S.J. Lindenbaum, C.S. Chan, M.A. Kramer, T.J. Hallman, L. Madansky, B.E. Bonner, J.A. Buchanan, C.N. Chiou, J.M. Clement, M.D. Corcoran, J.W. Kruk, H.E. Miettinen, G.S.

Mutchler, F. Nessi-Tedaldi, M. Nessi, J.B. Roberts). 810 future plans. Proc. of the Workshop on Heavy Ion Physics at AGS, Brookhaven National Laboratory, March 5-7, 1990, Ole Hansen, Editor, p. 227-239 (BNL 1990).

T. Hallman, et al., First observation of $A^{0}, \bar{\Lambda}^{0}$ and $K^{o}$ s production in relativistic heavy ion collisions at the AGS. Proc. of the Workshop on Heavy Ion Physics at AGS, Brookhaven National Lab., March 5-7, 1990, O. Hansen ed., p. 182-192 (BNL 1990).

Lindenbaum, R.S. Longacre, W.A. Love, L. Madansky, T.W. Morris, G. S. Mutchler, E. Nessi-Tedaldi, M. Nessi, E.D. Platner, J.B. Roberts, A.C. Saulys, and K. Zhao). Studies of neutral Vee production by Si ions at $14.5 \times A$ $\mathrm{GeV} / \mathrm{c}$ in $\mathrm{Au}$ and $\mathrm{Cu}$. Proc. of the 25th Intern. Conf. on High Energy Physics, Singapore, August 2-8, 1990, K.K. Phua and Y. Yamaguchi, Eds., p. 1092-1093 (South East Asia Theoretical Physics Assoc., Physical Society of Japan and co-sponsoring associations).

William A. Love (for the E810 Collaboration: B.E. Bonner, J.A. Buchanan, C.S. Chan, C.N. Chiou, J.M. Clement, M.D. Corcoran, S.E. Eiseman, A. Etkin, K.J. Foley, R.W. Hackenburg, T.J. Hallman, M.A. Kramer, J.W. Kruk, S.J. Lindenbaum, R.S. Longacre, W.A. Love, L. Madansky, T.W. Morris, G.S. Mutchler, M. Nessi, F. NessiTedaldi, E.D. Platner, J.B. Roberts, A.C. Saulys, K. Zhao. Silicon ion inter-actions measured in the E810 TPC at the AGS. Proc. of QUARK MATTER 90, Menton, France, May 7-11, 1990, J.P. Blaizot, C. Gerschel, B. Pire, A. Romana, Eds. Nuclear Physics A525, 601c-604c (1991).

Matthew A. Bloomer, William Love, Laurie Waters. New results from AGS heavy-ion experiments. Proc. of QUARK MATTER 90, Menton, France, May 7-11, 1990, J.P. Blaizot, C. Gerschel, B. Pire, A. Romana, Eds. Nuclear Physics A525, 601c-604c (1991).

S.E. Eiseman, A. Etkin, K.J. Foley, R.W. Hackenburg, R.S. Longacre, W.A. Love, T.W. Morris, E.D. Platner, A.C. Saulys, S.J. Lindenbaum, C.S. Chan, M.A. Kramer, K. Zhao, T.J. Hallman, L. Madansky, B.E. Bonner, J.A. Buchanan, C.N. Chiou, J.M. Clement, G.S. Mutchler, J.B. Roberts. An experiment to observe strange particle production in ion collisions at the AGS. High Engery Nuclear Collisions \& Quark Gluon Plasma. M.Biyajima, H. Enyo, T. Kunihiro, O. Miyamura, Eds., Proc. of the Intern. Symposium on High Energy Nuclear Collisions and Quark Gluon Plasma, Kyoto, Japan, June 6-8, 1991 p. 62-68. 
S.J. Lindenbaum (for the E-810 Collaboration: D.L. Adams, S. Ahmad, B.E. Bonner, J.A. Buchanan, C.S. Chan, C.N. Chiou, J.M. Clement, M.D. Corcoran, S.E. Eiseman, T. Empl, A. Etkin, K.J. Foley, R.W. Hackenburg, T.J. Hallman, M.A. Kramer, S.J. Lindenbaum, R.S. Longacre, W.A. Love, L. Madansky, H.E. Miettinen, T.W. Morris, G.S. Mutchler, E.D. Platner, J.B. Roberts, A.C. Saulys, J. Skeens, K. Zhao, Y. Zhu. Results on search for a $Q G P$ with a TPC magnetic spectrometer at AGS and plans for an 4 TPC magnetic spectrometer at RHIC. Advances in Nuclear Dynamics NAMICS (Proc. of the 7th Winter Workshop on Nuclear Dynamics, Key West, Fla., Jan. 26Feb. 2, 1991). W. Bauer and J. Kapusta, Eds. p. 68-78 (World Scientific, 1991).

A. Etkin, et al., Behavior of TPC's in a high particle flux environment. Proc. of the Symposium on RHIC Detector R\&D, Brookhaven National Laboratory, October 10-11, 1991. Y. Makdisi and A.J. Stevens, Eds. p. 207-213 (BNL, 1991).

S. Ahmad, et al., A silicon multiplicity detector system for an experiment on the interaction of antiprotons with nuclei at BNL. Conf. Record of the 1991 IEEE Nuclear Science Symposium and Medical Imaging Conf., Nov. 29, 1991, Santa Fe, NM, Vol.1, p. 377-380. (1991); and IEEE Transactions on Nuclear Science, Vol. 39, No. 4, 615-618 (1992).

S. Ahmad, et al., Transverse momentum distributions of $\pi^{*}$ from $14.6 \times \mathrm{A} \mathrm{GeV/c} \mathrm{silicon} \mathrm{ion} \mathrm{interactions} \mathrm{in} \mathrm{copper}$ and gold. Phys. Lett. B281, 29-32 (1992).

L. Madansky, et al., Recent results from E810. (Proc. of Quark Matter 1991.) Nuclear Physics A544, 335c-342c, (1992).

S.E. Eiseman, et al., Rapidity distributions and nuclear transparency in heavy ion collisions. Phys. Lett. B292, 10-12 (1992).

A. Etkin, et al., Effects of high beam rates on TPC's. Proc. of the 6th Wire Chamber Conf., Vienna, Austria, Feb. 17-21, 1992), Nucl. Instruments and Methods in Physics Research A323, 224-227, (1992)

A. Etkin, et al., Behavior of TPC's in a high particle flux environment, Conf. Record of the 1991 IEEE Nuclear Science Symposium and Medical Imaging Conf., November 2-9, 1991, Santa Fe, NM. Vol. 1. p. 537-541 (1991): and IEEE Transactions on Nuclear Science, Vol. 39 No. 4, 696-700, (1992).

S.J. Lindenbaum (for the E810 Collaboration: A. Etkin, S.E. Eiseman, K.J. Foley, R.W. Hackenburg, R.S. Longacre, W.A. Love, T.W. Morris, E.D. Platner, A.C. Saulys, S.J. Lindenbaum, T.J. Hallman, C.S. Chan, E. Efstathiadis, M.A. Kramer, K. Zhao, Y. Zhu, L. Madansky, S. Ahmad, B.E. Bonner, J.A. Buchanan, C.N. Chiou, J.M. Clement, G.S. Mutchler). Rapidity distributions of $K_{s}$ and $\Lambda$ 's produced by $14,6 \mathrm{GeV} / \mathrm{c}$ Si beams on Si and $\mathrm{Pb}$ targets. Presented at the 26th Intern. Conf. on High Energy Physics (ICHEP 92), August 6-12, 1992, Dallas, Texas.

S.E. Eiseman, et al., Rapidity distributions of $\mathrm{K}_{\mathrm{s}}^{\circ}$ 's and $\Lambda$ 's produced by $14.6 \times \mathrm{A} \mathrm{GeV/C} \mathrm{Si} \mathrm{beams} \mathrm{on} \mathrm{Si} \mathrm{and} \mathrm{Pb}$ targets, Phys. Lett. B297, 44-48, (1992).

811 E.C. Booth, et al., A study of radiative hyperon processes at Brookhaven, XI Intl. Conf. on Particles and Nuclei, Kyoto, 20-24 April 1987.

D.A. Whitehouse, et al., Radiative kaon capture in hydrogen, Bull. Am. Phys. Soc. 33, 1022 (1988).

K.P. Gall, et al., Radiative kaon capture in deuterium, Bull. Am. Phys. Soc. 33, 1022 (1988)

B.L. Roberts, et al., $\Sigma^{+}$Weak radiative decay, Bull. Am. Phys. Soc. 33, 1022 (1988).

B.L. Roberts, et al., Radiative kaon capture and hyperon weak radiative decay, Nucl. Phys. A479, 75c (1988). 
A.J. Noble, et al., $\Lambda$ and $\Sigma^{+}$weak radiative decay, Proc. of the Third Conf. on the Intersection of Particle and Nuclear Physics, Rockport ME, 14-19 May, 1988, AIP Conf. Proc. 176, 842 (1988).

E.K. McIntyre, et al., Radiative kaon capture, Proc. of the Third Conf. on the Intersection of Particle and Nuclear Physics, Rockport ME, 14-19 May 1988, AIP Conf. Proc. 176, 673 (1988).

D. Horváth, et al., Experimental study of radiative hyperon processes following kaon capture on the proton, Int. Conf. on Mesons \& Light Nuclei, Bedujné, Czechoslovakia, August 1988.

E.K. McIntyre, et al., Radiative kaon capture in hydrogen, Excited Baryons 88, August 1988, RPI, Troy, N.Y.

D. Horváth, et al., Experimental study of radiative hyperon processes following kaon capture on the proton, $\mathrm{J}$. Phys. B, 160 (1989).

D. Horváth, et al., Radiative hyperon processes following kaon capture on protons, Intl. Seminar on Intermediate Energy Physics, Moscow, USSR, November 17-30, 1989.

E.K. McIntyre, et al., Radiative kaon capture in hydrogen, Excited Baryons 1988, Proc. editor G. Adams, N.C. Mukhopadhyay and P. Stoler, World Scientific, 434-438 (1989).

N.P. Hessey, et al., A measurement of the branching ratio for the $\Sigma^{+} p \gamma$ decay, Z. Physik, C42, 175 (1989).

D.A. Whitehouse, et al., Radiative kaon capture at rest on the proton, Phys. Rev. Lett. 63, 1352 (1989).

B.L. Roberts, et al., Radiative hyperon processes, Nuova Cim. 102 A, N. 1, 145 (1989).

B.L. Roberts, Radiative hyperon decay, Excited Baryons 1988, Proc. ed. G. Adams, N.C. Mukhopadhyay and P. Stoler, World Scientific, 410-410 (1989).

K.P. Gall, et al., Radiative kaon capture on deuterium and the An scattering lengths, Phys. Rev. C, Rapid Comm., 42, R475 (1990).

A.J. Noble, et al., $A$ study of the weak radiative decay $A \rightarrow n \gamma$, DPF90 Conf., Houston, January 1990, Bull. Am. Phys. Soc., 35, 1207 (1990).

M.D. Hasinoff, et al., The reaction $\pi^{\circ} p \rightarrow \pi^{0} \pi^{0} n$ near threshold, DPF90 Conf., Houston, Jan. 1990, Bull. Am. Phys. Soc. 35, 1209 (1990).

B. Bassalleck, et al., The weak radiative decay $\Lambda \rightarrow n+\gamma$, a status report from Brookhaven experiment 811 , contribution to the 18th INS Int. Symposium on Physics with High-Intensity Hadron Accelerators, Tokyo, March 14-16, 1990.

K.D. Larson, et al., A report on the measurement of the weak radiative decay $\Lambda \rightarrow n+\gamma$, Proc. of the International Conf. on Particles and Nuclei, MIT, June 1990; abstract V-29.

B.L. Roberts, et al., Weak radiative hyperon decays, Int. Symp. on Weak Interactions and Neutrino Physics, Ginosar, Israel, April 1989, Nucl. Phys. B13, 449 (1990).

A.J. Noble, et al., Measurement of the $A \rightarrow n \gamma$ branching ratio, Phys. Rev. Lett. 69, 410 (1992).

813 P. D. Barnes (for the E813 collaboration), Search for the H particle: its production and weak decay, LA-UR-92535-mc (microfiche), Dec., 1991. Intl. Symp. On Hypernuclear and Strange Particle Physics, Simoda, Japan, Dec. 9-12, 1991, Nucl. Phys. A547:3c-16c, 1992.

P.H. Pile, et al., A New 1-2 GeV/c separated beam for BNL. Nucl. Inst. and Methods in Physics Research A321, 48-58, (1992). 
B.P. Quinn et. al., The search for the $H$ dibaryon with the $B N L 2.0 \mathrm{GeV} / \mathrm{c}$ kaon beam, Invited Talk presented at the 4th Conf. on the Intersections between Particle and Nuclear Physics, May 24-29, 1991, Tucson, Arizona. AIP Conf. Proc. 243, p. 579-581 (1992).

B.P. Quinn, et. al., Search for the $H$ particle with the Brookhaven $2.0 \mathrm{GeV}$ kaon beam, invited talk presented by B. Quinn at the Intl. Conf. on the Structure of Baryons and Related Mesons Workshop on Future Directions in Nuclear and Particle Physics at Multi-GeV Hadron Beam Facilities, March 4-6 (1993). BNL-52389, p. 340 (1993) held at Yale U., June 1-4, 1992, pub. by World Scientific and edited by M. Gai, p. 278-287.

V. Sum, ct al., A Time-of-flight array for 1 to $2 \mathrm{GeV} / \mathrm{c}$ particles. Nucl Inst. and Methods in Physics Research, A326 489-495, North-Holland (1993):

G.B. Franklin, et. al., Strange dibaryons, invited talk presented by G. Franklin at the Workshop on Future Directions in Nuclear and Particle Physics at Multi-GeV Hadron Beam Facilities, March 4-6 (1993). BNL--52389, p. 340 (1993).

M. Athanas, et. al., Search for the H dibaryon by Xi- capture on the deutron, Proc. of the 13 th Intl. Conf. on Particles and Nuclei held in Perugia, Italy, June28-July 2, 1993, pub. by World Scientific and edited by Alessandro Pascolini, p. 652-655.

Toru Iijima, H-dibaryon search in the reaction $\Xi^{-} d \rightarrow H n$ at rest by measuring the neutron in coincidence with the $\Xi$, Memoirs of the Faculty of Science, Kyoto University, Series A of Physics, Astrophysics, Geophysics and Chemistry, Vol. XXXIX, No. 2, Article 1, 1995.

B.P. Quinn, et. al., The search for the $H$ dibaryon with the BNL $2.0 \mathrm{GeV} / \mathrm{c}$ kaon neam. Proc. of the 5th Conf. on the Intersections between Particle and Nuclear Physics, May, 1994. AIP conf. proc. 338, p. 520-525 (1995).

F. Merrill, H-dibaryon search via $\Xi^{-}$capture on the deuteron, UMI-96-05051-mc(microfiche), June 1995, 213, $\mathrm{Ph} . \mathrm{D}$. Thesis (Carnegie Mellon U.).

Robert E. Chrien, H particle searches at Brookhaven. Proc. Int. Conf. Quark Lepton Nuclear Physics, Osaka, Japan, May 20-23, 1997.

Gan, Liping, A study of the sensitivity of the $H$ dibaryon search experiment 813 at $B N L$ through $\Sigma^{-}, p_{\text {atom }} \rightarrow A+n$, PhD thesis, University of Manitoba, 1998.

F. Merrill, et al., "H-dibaryon search via $\Xi$ ' capture on the deuteron", Phys. Rev. C., 63, pp. 035206-7, 2001.

814 B. Bassalleck, et al., Transverse energy distributions in Si-nucleus collisions at 10 GeV/nucleon. Proc. Quark Matter 1987 Conf., Z. Physik, C38 (1988) 45, H.J. Specht, editor.

S.V. Greene, et al., Search for strange matter in relativistic heavy ion collisions, Proc. Nato Advanced Study Inst. on the Nuclear Equation of State, May 1989, Peniscola, Spain, W. Greiner and H. Stoecker, editors, Nato ASI Series Vol 216B (1989) 117.

M. Fatyga, D. Makowiecki and W.J. Llope, Fast Monte Carlo simulation of U/Cu/Sci calorimeters, Nucl. Inst. Meth. A284, 323, (1989).

J. Barrette, et al., Electromagnetic dissociation of ${ }^{28} \mathrm{Si}$ at $E_{\text {lab }} / \mathrm{A}=14.6 \mathrm{GeV}$ by nucleon emission, Phys. Rev. C41, 1512, (April 1990).

J. Barrette, et al., Energy flow and stopping in relativistic heavy ion collisions at $E_{\text {lab }} / A=14.6 \mathrm{GeV}$, Phys. Rev. Lett. 64, 1219, (1990).

J. Barrette, et al. Search for strange quark matter in high-energy heavy ion collisions, Phys. Lett. B 252, 550, (1990). 
R. Debbe, et al., A study of wire chambers with highly segmented cathode pad read-out for high multiplicity charged particle detection, IEEE Trans. Nucl. Sci. 37, 82, (1990).

R. Bellwied, et al., Baryon spectra in the low Pt region, Proc. Workshop on Heavy Ion Physics at the AGS (HIPAGS), March 1990, O. Hansen, Editor, BNL-44911.

B. Shiva Kumar, et al., Global observables in Si-nucleus collisions at $14.6 \mathrm{GeV} /$ nucleon, Proc. Workshop on Heavy Ion Physics at the AGS (HIPAGS), March 1990, O. Hansen, editor, BNL-44911.

J. R. Hall, et al., Charged particle multiplicity in relativistic heavy ion collisions, Proc. Workshop on Heavy Ion Physics at the AGS (HIPAGS), March 1990, O. Hansen, editor, BNL-44911.

M. Fatyga, et al., Studying extremely peripheral collisions of relativistic heavy ions, Proc. Workshop on Heavy Ion Physics at the AGS (HIPAGS), March 1990, O. Hansen, editor, BNL-44911.

J. Fischer, et al., A many particle tracking detector with drift planes and segmented cathode read-out, IEEE Transactions on Nuclear Science 37, 88, (1990).

W.J. Llope and P. Braun-Munzinger, Electromagnetic dissociation of relativistic heavy ions, Phys. Rev. C (April 1990).

J. Simon-Gillo, et al., PCal construction, Nucl. Instr. Meth. A309, 427, (1991).

W.E. Cleland, et al., Energy flow and nucleon spectra in central collisions of $14.6 \mathrm{GeV} /$ nucleon Si with $\mathrm{Al}$, Cu, and Pb, Proc. Quarkmatter 90, Menton, Nucl. Phys. A525, 91c, (1991).

T.K. Hemmick et al., Production of exotic objects, Proc. Quarkmatter 90, Menton, Nucl. Phys. A525, 369c, (1991).

M.A. Bloomer, W.A. Love and L. Waters (for the E802, E810, and E814 collaborations), Recent results from relativistic heavy ion experiments at the AGS, Proc. PANIC XX, MIT, June 1990, Nucl. Phys. A527, 595c, (1991.

F. Rotondo, et al., Search for strange quark matter, Nucl. Phys. B24 (Proc. Suppl.), 265, (1991).

R. Bellwied, et al., Forward baryons in $14.6 \mathrm{GeV} /$ nucleon heavy ion collisions, Proc. 7th Winter Workshop on Nuclear Dynamics, Key West, Jan. 1991, J. Kapusta and W. Bauer, editors.

B. Shiva Kumar, et al., What can we learn from studies of proton and antiproton distributions at AGS energies? Proc. 7th Winter Workshop on Nuclear Dynamics, Key West, Jan. 1991, J. Kapusta and W. Bauer, editors.

J. Stachel, et al., (for the E814 collaboration), Global variables and forward baryons in $14.6 \mathrm{GeV} /$ nucleon Sinucleus collisions, Proc. 4th Int. Conf. Nucleus-Nucleus Collisions, Kanazawa, Japan, June 1991, Nucl. Phys. A.

J. Stachel, et al., (for the E814 collaboration), Global variables and forward baryons in $14.6 \mathrm{GeV} / \mathrm{nu}$-cleon Sinucleus collisions, Proc. Int. Symposium on High Energy Nuclear Collisions and Quark-Gluon Plasma, Kyoto, Japan, June 1991, T. Kunihiro, editor, World Scientific 1992, p. 167.

J. Barrette et al, Charged particle multiplicity in ${ }^{28} \mathrm{Si}+\mathrm{Al}, \mathrm{Cu}, \mathrm{Pb}$ reactions at $E_{\text {lab }}=14.6 \mathrm{GeV} /$ nucleon, Phys. Rev. C46, 312, (1992).

J. Barrette, et al., Excitation energy distribution of relativistic ${ }^{28}$ Si in electromagnetic dissociation into $p+{ }^{27} \mathrm{Al}$, Phys. Rev. C45, 2427, (1992).

P. Braun-Munzinger (for the E814 Collaboration), Recent results from Exp. 814 at Brookhaven, Quark Matter 91 Conf., Gatlinburg, Nov. 1991, Nucl. Phys. A544, 137c, (1992). 
M.S. Muthuswamy (for the E814 collaboration), Momentum distributions of light mass fragments in Si-nucleus collisions at 14.6 GeV/nucleon, Quark Matter, 91 Conf., Gatlinburg, Nov. 1991, Nucl. Phys. A544, 423c, (1992).

S.V. Greene (for the E814 Collaboration), Antiproton production in ${ }^{28}$ Si-nucleus interactions, Quark Matter 91 Conf., Gatlinburg, Nov. 1991, Nucl. Phys. A544, 599c, (1992).

D. Fox, et al., Response of the participant calorimeter to 1.5-6.8 GeV/c electrons and hadrons, Nucl. Instr. Meth. A317, 474, (1992).

S. J. Bennett (for the E814 Collaboration), Preliminary results from the E814 target rapidity telescope, HIPAGS 3 Symposium, Boston, MITLNS-2158, p. 333, January 1993, S. Steadman editor, BNL-49943.

S. Voloshin (for the E814 Collaboration), Two-particle correlations in the Si+A collisions at $14.6 \mathrm{~A} \mathrm{GeV/c}$, HIPAGS 3 Symposium, Boston, MITLNS-2158, p. 406, January 1993, S. Steadman editor, BNL-49944.

Z. Zhang (for the E814 Collaboration), Transverse energy production in collisions with $1 l .4 \mathrm{~A} \mathrm{GeV/c} \mathrm{Au} \mathrm{beam,}$ HIPAGS 3 Symposium, Boston, MITLNS-2158, p. 30, Jan. 1993, S. Steadman, editor, BNL-49933

T. Hemmick (for the E814 Collaboration), Hadron production at low $P_{\perp}$ in E814, HIPAGS 3 Symposium, Boston, MITLNS-2158, p. 204, Jan. 1993, S. Steadman, editor, BNL-49939.

J. Dee (for the E814 Collaboration), Baryon density and stopping in E814, HIPAGS 3 Symposium, Boston, MITLNS-2158, p. 47, Jan 1993, S. Steadman, editor, BNL-49940.

N. Xu (for the E814 Collaboration), Two-particle correlations from Si+Pb collisions at $14.6 \mathrm{~A} \mathrm{GeV/c}$, HIPAGS 3 Symposium, Boston, MITLNS-2158, p. 362, Jan. 1993, S. Steadman, editor, BNL-49941.

S. Kumar (for the E814 Collaboration), Antiproton production and annihilation in Si+A collisions at $14.6 \mathrm{~A}$ GeV/c, HIPAGS 3 Symposium, Boston, MITLNS-2158, p. 144, Jan. 1993, S. Steadman, editor, BNL-49967.

T. K. Hemmick (for the E814 Collaboration), Hadron production and correlations from E814, Proc. XXII Winter Workshop on Nuclear Dynamics, Key West, Florida, Feb. 1993, B. Back, editor.

J. Barrette (for the E814 Collaboration), Transverse energy production with Si and Au beams at AGS energy: towards hot and dense hadronicm matter, Proc. Quarkmatter 93. Nucl. Phys. A566, 411c, (1993).

J. Stachel (for the E814 Collaboration), Particle spectra and correlations from experiment 814, Proc. Quarkmatter 93, Nucl. Phys. A566, 183c, (1994).

T. K. Hemmick (for the E814 Collaboration), Low pt pion enhancement in ${ }^{28} \mathrm{Si}+\mathrm{Pb}$ collisions at $14.5 \mathrm{~A} \mathrm{GeV/C}$, Proc. Quarkmatter 93, Nucl. Phys. A566, 435c, (1994).

M. Rosati (for the E814 Collaboration), Particle production in $p+A$ collisions at $14.6 \mathrm{GeV} / \mathrm{c}$, Proc. Quark-matter 93, Nucl. Phys. A566, 597c, (1994).

N. Xu (for the E814 Collaboration), Pion interferometry in ${ }^{28} \mathrm{Si}+\mathrm{Pb}$ central collisions, Proc. Quarkmatter 93 , Nucl. Phys. A566, 585c, (1994).

J. Barrette, et al., (for the E814 collaboration), Two charged particle and transverse energy correlations in $\mathrm{Si}+\mathrm{Pb}$ collisions at 14.6 A GeV/c, Phys. Rev. C49, 1669, (1994).

Z. Zhang, P. Braun-Munzinger, W. Cleland, G. David, D. Lissauer, Response matrix approach to the Analysis of Calorimetry Data, Nucl. Instr. Meth. A 343, 610, (1994). 
P. Braun-Munzinger, (for the E814 Collaboration), Compression, expansion and freeze-out, in "hot and dense nuclear matter", Plenum Press, New York 1994, p. 419, W. Greiner, H. Stöcker, and A. Gallmann, eds.

G. David, et al., Prototype tests of a high resolution electromagnetic calorimeter using undoped CsI crystal, Nucl. Inst. Meth. Phys. Res. A348, 87, (1994).

J. Barrette, et al., (for the E814 Collaboration), Production of light nuclei in relativistic heavy ion collisions, Phys. Rev. C50, 1077, (1994).

J. P. Wessels and Y. C. Zhang, (for the E877 Collaboration), Is there flow at the AGS?, Proc. 10th Winter Workshop on Nuclear Dynamics, Snowbird, Utah, Jan. 1994, W. Bauer, editor.

N. Xu, (for the E814 Collaboration), Two-pion interferometry-towards equilibrium at the AGS, Proc. 10th Winter Workshop on Nuclear Dynamics, Snowbird, Utah, Jan. 1994, W. Bauer, editor.

J. Barrette, et al., (for the E814 collaboration), Centrality dependence of longitudinal and transverse baryon distributions in ultra-relativistic nuclear collisions, Phys. Rev. C50, 3047, (1994).

J. Barrette, et al., (for the E814 collaboration), Evidence for expansion of a hot fireball from two-pion correlations for $S i+P b$ collisions at AGS energy, Phys. Lett. B333, 33, (1994).

J. Barrette, et al., (for the E814 collaboration), Electromagnetic dissociation of relativistic ${ }^{28}$ Si, Phys. Rev. C51, $865,(1995)$.

J. Barrette, et al., (for the E814 collaboration), Measurement of pion enhancement at low transverse momentum and of the resonance abundance in Si-nucleus collisions at the AGS, Phys. Lett. B351, 35, (1995).

815 K. B. Bhalla, et al., Particle densities and nuclear breakup in ${ }^{16}$ O-emulsion interactions, Proc. Int. Euro-physics Conf. on High Energy Physics, Uppsala, Sweden, June 25-July 1, 1987, Vol. I, p. 155. (Petit-Lancy, Switzerland: Eur. Phys. Soc., 1987).

H. H. Heckman, et al., Nuclear breakup and particle densities in $200 \mathrm{~A} \mathrm{GeV}-{ }^{16} \mathrm{O}$ interactions with emulsion nuclei, Proc. of XVIII Int. Symp. on Multiparticle Dynamics, Tashkent, USSR, Sept. 8-12, 1987, p. 411-420. (World Scientific, Singapore, 1988, Eds.: I. Remin and K. Gulamov.)

E. Monnand, et al., Pseudorapidity densities in central ${ }^{16} \mathrm{O}$ interaction at $200 \mathrm{~A} \mathrm{GeV}$, Proc. of theWorkshop held at Moscow, USSR, Aug. 1987, during the $20^{\text {th }}$ Int. Cosmic Ray Conf., Moscow, Lebedev, 1988, p. 4-10.

M.I. Adamovich, et al., Multiplicities and rapidity in $200 \mathrm{~A} \mathrm{GeV}{ }^{16} \mathrm{O}$ interactions with emulsion nuclei, Phys. Rev. Lett. B201, 397 (1988).

S. Garpman, et al., A computer-based coordinate measuring station for nuclear emulsion chambers, Nucl. Instr. Meth. A269, 134 (1988).

S. Lokanathan, et al., The EMU-01 experiment: A status report, Proc. Int. Conf. on Physics and Astrophysics of Quark Gluon Plasma, Bombay, India, February 8-12, 1988, p. 514-522. (World Scientific, Singapore, 1989, Eds: B. Sinha and S. Raha.)

R. J. Wilkes, et al., Pseudorapidity distributions and correlations in central ${ }^{16} \mathrm{O}$ interactions at $200 \mathrm{~A} \mathrm{GeV}$, Proc. 3rd Conf. on the Intersections between Particle and Nuclear Physics, Rockport, ME, May 14-19 1988. AIP Conf. Proc. (USA) 176, 1047. G. Bunce, editor. (1988).

M.I. Tretyakova, et al., Central interactions of ${ }^{16} \mathrm{O}$ nuclei with heavy nuclei of photoemulsion at $200 \mathrm{GeV} /$ nucleon, P. N. Lebedev Physical Institute Reprint 244 (1988). Proc. IX Int. Seminar on High Energy Physics ProblemsRelativistic Nuclear Physics and Quantum Chromodynamics, Dubna, June 1988. 
E. Friedlander, et al., $A$ search for non-statistical fluctuations and structures in the rapidity density distri-bution of ${ }^{15} \mathrm{O}+\mathrm{Ag}(\mathrm{Br})$ and ${ }^{32} \mathrm{~S}+\mathrm{Au}$ interactions at 60 and $200 \mathrm{~A} \mathrm{GeV}$. Proc. Hadronic Matter in Collision, Tucson, Arizona, Oct. 6-12, 1988, p. 210-221. (World Scientific, Singapore, 1989, Eds: B P. Carruthers and J. Rafelski.)

E. Stenlund, et al., A search for non-statistical particle density fluctuations in ${ }^{16} \mathrm{O}+\mathrm{Ag}(\mathrm{Br})$ and ${ }^{32} \mathrm{~S}+\mathrm{Au}$ interactions at $200 \mathrm{~A} \mathrm{GeV}$, Proc. 7th Int. Conf. on Ultra-Relativistic Nucleus-Nucleus Collisions - Quark Matter 1988, Lennox, Massachusetts, September 26-20, 1988, Nucl. Phys. A 498, 541 c, (1989).

M.I. Adamovich, et al., Scaling properties of charged particle multiplicity distributions in oxygen induced emulsion interactions at 14.6, 60 and $200 \mathrm{~A}$ GeV, Phys. Lett. B, 223, 262 (1989).

M.I. Adamovich, et al., Limiting fragmentation in oxygen-induced emulsion interactions at 14.6, 60 and 200 GeV/nucleon, Phys. Rev. Lett. 62, 2801 (1989).

M.I. Adamovich, et al., Production of helium $(Z=2)$ projectile fragments in ${ }^{16} O$-emulsion interactions from $E / A=2$ to $200 \mathrm{GeV}$, Phys. Rev. C 40, 66 (1989).

M.I. Adamovich, et al., A study of recoil protons in ultra-relativistic nucleus-nucleus collisions, Phys. Lett. B 230 , 175 (1989).

M.I. Adamovich, et al., Charged particle spectra in oxygen-induced reactions at 14.6 and $60 \mathrm{GeV} /$ nucleon, High Energy Physics and Nuclear Physics in China 13 (1989) 341; 13, 865, (1989). [English and Chinese.]

M.I. Adamovich, et al., Rapidity densities and their fluctuations in central $200 \mathrm{~A} \mathrm{GeV}{ }^{32}$ S interactions with Au and Ag, Br. nuclei, Phys. Lett. 227B, 285 (1989).

S. Persson, Measurement and three-dimensional reconstruction of particle tracks in emulsion chambers, Comp. Phys. Comm. 55, 103 (1989).

E. Stenlund et al., A search for non-statistical particle density fluctuations in ${ }^{16} \mathrm{O}+\mathrm{Ag}(\mathrm{Br})$ and ${ }^{32} \mathrm{~S}+\mathrm{Au}$ interactions at $200 \mathrm{~A} \mathrm{GeV}$, Nucl. Phys. A498, 541c (1989).

E. Ganssauge et al., A Track Reconstruction Program (TRP) for evaluation of nucleus-nucleus collisions in nuclear track emulsion chambers, Comp. Phys. Comm. 55, 233, (1989).

M. I. Adamovich et al., On the energy and mass dependence of the multiplicity in relativistic heavy ion interactions, Modern Physics Letters A 5, 169 (1990).

I. Otterlund, et al., Stochastic emission and non-statistical fluctuations in relativistic heavy-ion interactions, Proc. X Int. Seminar on High Energy Physics Problems-Relativistic Nuclear Physics and Quantum Chromodynamics, Dubna, Sept. 1990.

E. Ganssauge, et al., Contribution of the EMU01 collaboration to the research for a Quark Gluon Plasma $(Q G P)$, Proc. XV Int. Conf. on Particle Tracks in Solids, Marburg, FRG, September 3-7, 1990.

M. I. Tretyakova, et al., Central interactions $S+\mathrm{AgBr}$ at $200 \mathrm{~A} \mathrm{GeV}$, Proc. X Int. Seminar on High Energy Physics Problems-Relativistic Nuclear Physics and Quantum Chromodynamics, Dubna, Sept. 1990, p. 412-419.

1. Otterlund, et al., Limiting fragmentation, scaling and substructural dependence of multiparticle production in high-energy heavy-ion interctions, Proc. 6th Nordic Meeting on Nuclear Physics. Kopervik, Norway, August 1989, Physica Scripta, 168, (1990).

M. I. Adamovich, et al., Target nucleus fragmentation in ${ }^{16} \mathrm{O}+(\mathrm{Ag}, \mathrm{Br})$ interactions at $200 \mathrm{~A} \mathrm{GeV}$, Phys. Lett. 234B, 180 (1990). 
M. I. Adamovich, et al., Scaled factorial moment analysis of $200 \mathrm{~A} \mathrm{GeV} \mathrm{sulphur} \mathrm{+} \mathrm{gold} \mathrm{interactions,} \mathrm{University} \mathrm{of}$ Washington Preprint UWSEA PUB 90-4 (Seattle, 1990), Phys. Rev. Lett. 65, 412, (1990).

M. I. Adamovich, et al., On the multiplicity fluctuations in relativistic heavy-ion collisions, Phys. Lett. 242B, 512, (1990).

E. Stenlund, et al., Recent results from EMU01, Proc. Workshop on Heavy Ion Physics at the AGS, HIPAGS90, Brookhaven National Laboratory; Upton, NY, March 5-7, 1990, p. 70, BNL-44911. (Ed.: O. Hansen.)

Liu Lianshou, et al., Energy, target, projectile and multiplicity dependence of intermittency behavior in highenergy $O(S i, S)$-emulsion interactions. Proc. XX Int. Symp. on Multiparticle Dynamics, Dortmund, FRG, September 13-18, 1990.

S. Garpman, et al., Rapidity fluctuations, cluster partitions and intermittency in relativistic heavy ion collisions, Nucl. Phys. A525, 551c, (1991).

I.Otterlund, et al., Extrapolations based on EMU01-data from oxygen, silicon and sulphur violent inter-actions at medium and high energies, Lund University Preprint LUIP 9105, Proc. Int. Symp. on High Energy Nuclear Collisions and Quark Gluon Plasma, Kyoto, Japan, June 6-8, 1991.

E. Stenlund, et al., Recent EMU01-results on fluctuations and intermittency, Lund University Preprint LUIP 9106, Proc. Ringberg Workshop on Multiparticle Production, Fluctuations and Fractal Structure, Ringberg Castle, Germany, June 25-28, 1991, p. 171-183. (World Scientific, Singapore, 1992, Eds.: R. C. Hwa, W. Ochs and N. Schmitz.)

M. M. Chernyavsky, et al., A direct analysis of intermittency-generating cascading in high energy nucleus-nucleus collisions, Proc. XXI Int. Symp. on Multiparticle Dynamics, Wuhan, China, Sept. 23-27, 1991. p. 442-445.

(World Scientific, Singapore, 1992. Eds.: Wu Yuanfang and Liu Lianshou.)

M. I. Adamovich, et al., Energy, target, projectile and multiplicity dependence of intermittency behavior in highenergy O(Si,S)-emulsion interactions, Z. Phys. C 49, 395, (1991).

M. I. Adamovich, et al., Stochastic emission of particles in ultra-relativistic heavy-ion collisions, Modern Physics Letters A 6, 469, (1991).

M. I. Adamovich, et al., Slow, target associated particles produced in ultra-relativistic heavy-ion inter-actions, Phys. Lett. 262B, 369, (1991).

I. Otterlund, et al., Multiplicity fluctuations in high-energy heavy-ion interactions observed in high-angular resolution tracking detectors, Proc. Santa Fe Workshop on Intermittency in High-Energy Collisions. Santa Fe, New Mexico, March 18-21, 1990, p. 126-44. (World Scientific, Singapore, 1991, Eds.: F. Cooper, R. C. Hwa, I. Sarcevic.)

J. Wilkes, et al., Scaled factorial moment analysis of $200 \mathrm{~A} \mathrm{GeV} \mathrm{S} \mathrm{+} \mathrm{Au} \mathrm{interactions.} \mathrm{Proc.} \mathrm{Santa} \mathrm{Fe} \mathrm{Work-shop}$ on Intermittency in High-Energy Collisions. Santa Fe, New Mexico, March 18-21, 1990, p. 145-157. (World Scientific, Singapore, 1991, Eds.: F. Conper, R. C. Hwa, I. Sarcevic.)

M. I. Adamovich, et al., The systematical investigation of intermittency in high energy heavy ion collisions, High Energy Physics and Nuclear Physics in China 15, 131, (1991). (Chinese)

E. Stenlund, et al., Non-statistical fluctuations in relativistic heavy-ion collisions.Proc. Int. Workshop on Correlations and Mutliparticle Production (CAMP) (LESIP IV), Marburg, F. R. Germany, May 14-16, 1990, p. 337-344. (World Scientific, Singapore, 1991, Eds.: M. Plümer, S. Raha, and R. M. Weiner.)

M. I. Adamovich, et al., On the systematic behavior of the intermittency-indices in nuclear interactions, Phys. Lett. 263B, 539, (1991). 
R. J. Wilkes, et al., Review of recent results on particle production from EMU01, Nucl. Phys. A544, 153c. (1992).

E. Stenlund, et al., Survey of recent EMU01-results from CERN/SPS and BNL/AGS. Proc. Joint Int. LeptonPhoton Symp. \& Europhysics Conf. on High Energy Physics, Geneva, Switzerland, July 25-August 1, 1991, Vol. 1, p. 478-481. (World Scientific, Singapore, 1992, Eds.: S. Hegarty, K. Potter and E. Quercigh).

M. I. Adamovich, et al., A systematical investigation of the energy independent behaviour in the fragmentation regions for ${ }^{16} \mathrm{O}+$ Em interactions from 3.7 to $200 \mathrm{~A} \mathrm{GeV}$, Z. Phys. C 55, 235, (1992).

M. I. Adamovich, et al., On intermittency in heavy ion collisions and the importance of $\square$-conversion in a multidimensional intermittency analysis, Nucl. Phys. B388, 3, (1992).

M. I. Adamovich, et al., Limiting fragmentation behaviour of correlation and fluctuation of particles produced in high energy oxygen-nucleus induced interactions, High Encrgy Physics and Nuclear Physics in China 16, 991, (1992).

M. I. Adamovich, et al., Local particle densities and global multiplicities in central heavy ion interactions at 3.7, 14.6, 60 and $200 \mathrm{~A} \mathrm{GeV,} \mathrm{Z.} \mathrm{Phys.} \mathrm{C56,} \mathrm{509,} \mathrm{(1992).}$

$\mathrm{H}$. Q. Wang, et al., Limiting fragmentation of two-particle correlation and dynamical fluctuation in O-Em interactions, Proc. XXI Int. Symp. on Multiparticle Dynamics, Wuhan, China, Sept. 23-27, 1991. p. 436-441. (World Scientific, Singapore, 1992, Eds.: Wu Yuanfang and Liu Lianshou)

M. I. Adamovich, et al., Rapidity density distributions in ${ }^{16 f} \mathrm{O},{ }^{28} \mathrm{Si},{ }^{32} \mathrm{~S},{ }^{197} \mathrm{Au}$ and ${ }^{208} \mathrm{~Pb}$ induced heavy ion interactions at 4 - $200 \mathrm{~A} \mathrm{GeV}$, Phys. Rev. Lett. 69, 745, (1992).

M.I. Adamovich, et al., Studies of angular distribution of helium projectile fragments in interactions of $200 \mathrm{~A} \mathrm{GeV}$ ${ }^{32} S$ ions with emulsion nuclei, Modern Physics Letters A 8, 21. (1993).

M. I. Adamovich, et al., Systematic investigation of scaled factorial cumulant moments for nucleus-nucleus interactions, Phys. Rev. D 47, 3726, (1993).

M. I. Adamovich, et al., On the jet-like and ring-like substructure in distributions of produced particles in central heavy-ion collisions at ultra-relativistic energies, Journal of Physics G - Nuclear and Particle Physics 19, 2035. (1993).

M. I. Adamovich, et al., Rapidity density distributions and their fluctuations in violent Au-induced inter-actions at 11.6 A GeV/c, Phys. Lett. B 322, 166, (1994).

J. Nystrand, et al., Rapidity density distributions in $A u+A u$ and $A u A g$ interactions at $11.6 \mathrm{~A} \mathrm{GeV/c}$, Nucl. Phys. A566. 419c, (1994).

M. I. Adamovich, et al., Relative information entropy of particle production in high energy induced nuclear reactions, High Energy Physics and Nuclear Physics in China 18 (1994) 61; 18, 291, (19949). (English and Chinese.)

M. I. Adamovich, et al., Local characters of final state multiplicity spectra in high energy heavy-ion central collisions, High Energy Physics and Nuclear Physics in China 18, 884, (1994). (Chinese.)

M. I. Adamovich, et al., Helium production in $10.7 \mathrm{~A} \mathrm{GeV} \mathrm{Au} \mathrm{induced} \mathrm{nucleus-nucleus} \mathrm{collisions,} \mathrm{Phys.} \mathrm{Lett.}$ B338, 397, (1994).

M. I. Adamovich, et al., On the production of slow particles in high-energy heavy ion collisions, Z. Phys. C 65 , 421, (1994).

M. I. Adamovich, et al., ${ }^{28} \mathrm{Si}\left({ }^{28} \mathrm{~S}\right)$ fragmentation at 3.7 A, $14.6 \mathrm{~A}$ and $200 \mathrm{~A} \mathrm{GeV}$, Z. Phys. A 351, 311, (1995). 
816 P. Astier, Search for neutrino oscillations, Proc. of BNL Neutrino Workshop, M. Murtagh, ed. (1987).

P. Astier, et al., Search for neutrino oscillations. Phys. Lett. 220B (1989), 646.

817 B.E. Bonner, et al., Spin transfer in hyperon production, Phys. Rev. Lett. 58,447 (1987).

B.E. Bonner, et al., Spin-parameter measurements in $\Lambda$ and $K_{s}$ production, Phys. Rev. D 38, 729 (1988).

B.E. Bonner, et al., Spin-parameter measurements in inclusive $\Sigma^{0}$ production, Phys. Rev. Lett. 62, 1591 (1989).

B.E. Bonner, et al., Analyzing power of inclusive production of $\pi^{+} \pi^{-}$, and $K_{\mathrm{s}}^{\circ}$ by polarized protons at 13.3 and $185 \mathrm{GeV} / \mathrm{c}$, Phys. Rev. D, 41, 13 (1990).

$818 \mathrm{~J} . \mathrm{H}$. Lee, et al., Spin parity analysis of the $f_{1}(1285) \pi^{-}$system in the reaction $\pi^{-} \rightarrow f_{1}(1285) \pi^{-} p$ at $1 \mathrm{GeV} / \mathrm{c}$, Phys. Lett. B323, 227-232 (1994).

$820 \quad K$. Johnston, et al., Search for a strangeness-1 dibaryon below the sigma $N$ threshold, Phys. Rev C46, pp. 15731576 (1992).

821 V. Hughes, The muon anomalous magnetic moment, Physica Scripta, T22, 111 (1988).

L. M. Barkov, et al., The anomalous magnetic moment of the muon, Proc. 9th Intl. Symposium on High Energy Spin Physics (1990).

B. L. Roberts, The new muon g-2 experiment at BNL, Proc. Intl. Symposium on the Future of Muon Physics (1991).

V. W. Hughes, The anomalous magnetic moment of the muon, Proc. Second Intl. Symposium on Particles, Strings and Cosmology, 868, Boston, MA, 25-30 March 1991. World Scientific, Pran Nath and Stephen Reucroft, eds.

G. T. Danby, W. Meng, W. B. Sampson, K. Woodle, Magnetic flux shielding for the precision muon g-2 storage ring superconducting inflector. Presented to 13th Intl. Conf. on Magnet Technology, Victoria, B. C. Canada (1993).

L. X: Jia, L. J. Addessi, J. R. Cullen, Jr., A. J. Esper, M. A. Green, R. E. Meier, C. Pai, L. P. Snydstrup, T. N. Tallerico, Cryogenics for the muon g-2 superconducting magnet system, Presented at the 15 th Intl. Cryogenic Engineering Conf., Genoa, Italy, Cryogenics, Vol. 34, ICEC Supp. 1994 p. $87-90$ (1994).

L. X. Jia, L. J. Addessi, J. R. Cullen, Jr., A. J. Esper, R. E. Meier, C. Pai, L. P. Snydstrup, Design parameters for gas-cooled electrical leads of the g-2 magnets. Presented at 15th Intl. Cryogenic Engineering Conf., Genova, Italy Cryogenics, Vol. 34, ICEC Supp. 1994 p. 631-634 (1994).

H. C. Hseuh, L. Snydstrup, M. Mapes. Beam vacuum system of Brookhaven's muon storage ring. Presented at 42nd National AVS Symposium, Minneapolis, MN, October 16-20, 1995 (BNL-61510).

L. Roberts, et al., Status of the new muon ( $g-2)$ experiment. Proc. of the Int. Conf. on High Energy Physics, Warsaw 1996.

J.P. Miller, et al., Status of the BNL muon g-2 experiment, Big Sky 1997, Intersections between particle and nuclear physics, pp. 792-800, 1997.

S. Sedykh, et al., Performance of the calorimeters for the muon ( $g$-2) experiment at the AGS, Tucson 1997, Calorimetry in high energy physics, pp. 269-275, 1997. 
Vernon W. Hughes (for the g-2 collaboration) et al., The anomalous magnetic moment of the muon, Workshop on Frontier Tests of Quantum Electrodynamics and Physics of the Vacuum, Sandansky, Bulgaria, June 1998.

R. M. Carey, et al., New measurement of the anomalous magnetic moment of the positive muon, Phys. Rev. Letters 82, pp 1632-1635 (1999)

Y.K. Semertzidis, et al., The muon g-2 experiment at BNL, Published in "Rochester 1999 Probing Luminos and Dark Matter" pp. $72-89$ (1999).

H. Brown, et al., Improved measurement of the positive muon anomalous magnetic moment", Phys. Rev. D62, pp. 91101 (2000).

K. Jungmann, et al., A new measurement of the muon magnetic anomaly", published in "Frascati 1999, Physics and Detectors for DAPHINE" pp. 547-557 (Nov. 1999).

H. N. Brown,et al., Precise measurement of the positive muon anomalous magnetic moment, Phys. Rev. Lett 86 , 2227 (2001).

825 J.B. Cumming, Y.Y. Chu, and P.E. Haustein. Momentum transfer in the reactions of $13.6-\mathrm{GeV} / \mathrm{nucleon}{ }^{16} \mathrm{O}$ with copper. Phys. Rev. C, 46, 2042-2046 (1992).

R.E. Chrien, et al. Search for bound states of the $\eta$-meson in light nuclei.Phys. Rev. Lett. 60, 2595-8,1988.

P.H. Pile, A search for bound states of the $\eta$-meson in light nuclei. Proc. 3rd Conf. on the Intersections Between Particle and Nuclear Physics, May 14-19, 1988, Rockport, ME. G.M. Bunce, Editor, AIP Conf. Proc. 176, 719724.

834 A.S. Carroll, et al. Nuclear transparency to large-angle pp elastic scattering, Phys. Rev. Lett, 61, 1698 (1988).

835 J. Alster, in Workshop on Nuclear Structure with Medium Energy Probes, Santa Fe, NM, 1988. AGS Experiment 835 Collaboration, Kaon-nucleus total cross sections, PANIC XII Intl. Conf. on Particles and Nuclei, Cambridge, MA. June 25-29, 1990.

Y. Mardor, et al., $K^{+}$total cross sections as a test for nucleon "swelling." Phys. Rev. Lett. 65, 2110-13 (1990).

Y. Mardor, $K^{+}$total cross-sections and swelling in nuclei. Thesis for M.Sc. degree at Tel-Aviv University (1990).

J. Alster (representing collaboration). $\mathrm{K}^{+}$- nucleus total cross section experiment and nuclear medium effects. Nucl. Phys. A547, 321c-30c (1992).

R.A. Krauss et, al., $K^{+}$total cross sections on ${ }^{12} \mathrm{C}$ and medium effects in nuclei, Phys. Rev. C 46, 655, (1992).

R. Sawafta (Exp. 835 Collaboration). The $K^{+}$meson as a probe of the nuclear medium. Invited talk. Fourth Conf. on the Intersections between Particle and Nuclear Physics, Tucson, Arizona, May 24-29, 1991, W.T.H. van Oers, Editor, AIP Conf. Series 243, 582-7 (1992).

R.E. Chrien, The $K^{+}$as a probe of nuclear medium effects. Invited talk, workshop on Strangeness in Nuclei, Krakow, Poland, May 5-7, (1992).

R. Sawafta, et al., The influence of the nuclear medium on $K^{+}$total cross sections. Phys. Rev. Lett. B307, 293 (1993).

R. Sawafta, et. al., What can be learned about meson nucleon interactions and nuclear structure from $K^{+}$total cross sections, invited paper presented at the 5th Intl. Symposium on Meson--Nucleon Physics and the Structure of the Nucleon. Sept. 6-10 (1993), Boulder. Colorado, piN Newsletter No. 8, 74-79, 1993. 
R. Weiss, et. al., Measurement of low energy $K^{+}$total cross sections on $N=Z$ nuclei, Phys. Rev. C49, 2569-2578 (1994).

E. Piasetzky, et. al., The measurements of nuclear medium effects with $\mathrm{K}^{+}$, invited talk Proc. of the Intl. Symposium on Medium Energy Physics, Beijing, China, August 1994.

E.Friedman, et al., $K^{+}$nucleus reaction and total cross sections: new analysis of transmission experiments, Phy. Rev. C, vol. 55, 3, pp. 1304 (1997).

836 R. W. Stotzer, et al., Search for the H-dibaryon in ${ }^{3} H e\left(K^{-}, K^{+}\right) H n$, Phys. Rev. Letts. 78, 3636 (1997).

838 C. White, et al., Comparison of 20 exclusive reactions at large t, Phys. Rev. D 49, 58 (1994).

840 Y. Semertzidis, et al., An experiment to produce light pseudoscalars and $Q E D$ vacuum polarization. Proc. of the Workshop on Cosmic Axions (BNL, 1989), C. Jones and A.C. Melissinos, eds., World Scientific, P. 137 (1990).

Y. Semertzidis, et al., Limits on the production of light scalar and pseudoscalar particles, Phys. Rev. Lett. 64, 2988 (1990).

Y. K. Semertzidis, Coherent production of light pseudoscalars (axions) inside a magnet field with a polarized laser beam; University of Rochester, Department of Physics and Astronomy, Rochester, NY. UR-1141, ER13065-603. January 17, 1991.

R. Cameron, et al., Measurement of the magnetic birefringence of neon gas, J. Opt. Soc. Am B8, 520 (1991).

R. Cameron, et al., A search for the coherent production of axions in the milli eV range, Particles and Fields 1991, $1002(1991)$.

R. Cameron, et al. First measurement of the magnetic birefringence of helium gas, Phys. Letts. A157, (1991).

D.M. Lazarus, et al., A search for solar axions, Phys. Rev. Lett. 69, 2333 (1992).

G. Ruoso, et al., Limits on light scalar and pseudoscalar particles from a photon regeneration experiment, University of Rochester, Department of Physics and Astronomy, Rochester, NY. UR-1248, ER-13065-697, January 1992.

G. Ruoso, R. Cameron, A.C. Melissinos, Y. Semertzidis, H.J. Halama, D.M. Lazarus, A.G. Prodell, F. Nezrick, C. Risso, E. Zavattini, Search for photon regeneration in a magnetic field, Zeits. fur Phys. C56, 505 (1992).

845 K.E. Ohl, et al., A measurement of the branching ratio and form factor for $K_{L} \rightarrow e^{+} e^{-}$, Phys. Rev. Lett., 65, 1407 (1990).

K.E. Ohl, et al., Improved experimental limit on $K_{L} \rightarrow \pi^{0} e^{+} e^{-}$. Phys. Rev. Lett. 64, 2755 (1990).

W.M. Morse, et al., Results from AGS E845: $\mathrm{K}_{\mathrm{L}}^{0} \rightarrow \pi^{0} e^{+} e^{-}, e^{+} e^{-} \gamma, e^{+} e^{-} e^{+} e^{-}$,"Proc. of the Conf. on the Intersections Between Particle and Nuclear Physics, Tucson, AZ, May 24-29, 1991, AIP Conf. Proc. 243.

W.M. Morse, et al., Search for the Decay $\mathrm{K}_{\mathrm{L}}^{0} \rightarrow \pi^{0} e^{+} e^{-}$, Nuc. Phys. A527, 717c-720c, (1991).

W.M. Morse, et al., Observation of the decay mode $\mathrm{K}_{\mathrm{L}}^{\circ} \rightarrow \gamma \gamma$ ee, Phys. Rev. D, 45, 36 (1992).

847 G. Singh, A.Z.M. Ismail and P.L. Jain, Characteristics of helium fragments produced in ${ }^{28}$ Si emulsion and interactions at $14.5 \mathrm{GeV}$, Phys. Rev. C, 43, 2417 (1991).

P.L. Jain, G. Singh, and K. Sengupta, Rapid communications, Phys. Rev. C., Vol. 43, No. 5 (1991). 
P.L. Jain, G. Singh, and K. Sengupta, Comparison of nucleus-nucleus interactions at $14.5-200 \mathrm{~A} \mathrm{GeV} \mathrm{with} \mathrm{a}$ Multistring Model, Phys. Rev. C43, R2027 (1991).

P.L. Jain and G. Singh, One-and two-dimensional analysis of intermittency in ultra-relativistic nucleus-Nucleus Interactions, Phys. Rev. C44, 854 (1991).

P.L. Jain, G. Singh, and K. Sengupta, Intranuclear cascading at ultrahigh energy in heavy-ion interactions, Z. Phys. C52, 465 (1991).

P.L. Jain and G. Singh, Investigation of intermittency in ${ }^{28} \mathrm{~S}$ i-nucleus collisions at $14.5 \mathrm{~A} \mathrm{GeV}$, Mod. Phys. Lett. A7, 93 (1992).

P.L. Jain and G. Singh, Intermittency in relativistic heavy-ion collisions, Z. Phys. C53, 355 (1992).

850 J.A. Appel, J. Botts, G. Bunce, G. Farrar, S. Pordes. Color Transparency Study Group.BNL-45319.

J. Y. Wu, et al., The EVA trigger: transverse momentum selection in a solenoid, NIM A349 (1994).

A. S. Carroll, et al., Measurement of color transparency by $C(p, 2 p)$ reaction at large momentum transfer, HADRON97 conf., BNL, Ed. S-U Chung (1997).

J. Aclander, et al., Short-range NN correlations: a direct measurement, HADRON97 conf., BNL, Ed. S-U Chung (1997).

I. Mardor, et al., High $p_{t}$ quasi-exclusive scattering with resonance production, 6th conf. on Intersection of Particle and Nuclear Physics (1997).

I. Mardor, Nuclear filtering in wide angle exclusive scattering, thesis, Tel-Aviv University, December 1997.

I. Mardor, et al., Nuclear transparency in large momentum transfer quasieleastic scattering, Phys. Rev. lett. 81, 5085 (1998).

Y. Mardor, et al., Measurement of quasi-elastic ${ }^{12} \mathrm{C}(p, 2 p)$ scattering at high momentum transfer, Phys. Lett B437, p.257-263 (1998).

J. Aclander, et al., A direct measurement of short-range $N N$ correlations in nuclei via the reactive ${ }^{12} C(p, 2 p+h)$, submitted to Phys. Rev Lett (1998).

Y. Mardor, et al., Quasi-elastic hadronic scattering at high momentum transfer, 6 th conf., on Intersections of Particle and Nuclear Physics (1997), thesis, Tel-Aviv University, April 1998.

$\mathrm{J}$. Aclander, et al., The large momentum transfer reaction $C-12(P, 2 P+N)$ as a new method for measuring short range $N N$ correlations in nuclei, Phys. Lett. B453, pp. 211-216 (1999).

A. Leksanov, et al., (for E850 collaboration), A new measurement of the energy dependence of nuclear transparency for large momentum transfer $12 C(p, 2 p)$ scattering, to be published in Proc. of CIPANP2000, May 2228, 2000, Quebec, Canada.

A. Tang, et al., (for E850 collaboration), $n$-p Short-range correlations from $(p, 2 p+n)$ measurements, to be published in Proc. of CIPANP2000, May 22-28, 2000, Quebec, Canada.

851 A. Deshpande, et al., Determination of the branching ratio of the decay $\pi^{\circ} \rightarrow e^{+} e^{-}$, DFT 1993, The Fermilab Meeting. 
852 A. Dzierba and S. Tiege for the E852 Collaboration, First results from the E852 tests of a 320-element lead-glass calorimeter. AGS Users Newsletter June 24, 1992.

B. Brabson, et al., A study of two prototype lead glass electromagnetic calorimeters. Nucl. Instr. \& Meth. A332, 419-443 (1993).

A.R. Dzierba, Meson spectroscopy with $\pi$ and $K$ beams, Proc. of the Workshop on Future Directions in Particle and Nuclear Physics at Multi-GeV Facilities, Brookhaven National Laboratory, March 4-6, 1993.

S. Y. Chung, Search for exotic mesons, invited talk at Workshop on Hadron Physics at $e^{+} e^{-}$Colliders, IHEP, Beijing, China, October 1994.

Z. Bar-Yam, et al, A cylindrical drift chamber of novel design. Nucl. Instrm. \& Methods A312, 398-410 (1995).

Z. Bar-Yam, et al, A scintillation detector of unique geometry. Nuclear Instrum. \& Methods, A357, 95-102 (1995).

S. U. Chung, Summary of Hadron95. Summary Talk given at the Sixth Intl. Conf. on Hadron Spectro-scopy, Manchester, England, July 9-14, 1995. (BNL-QGS-95-91)

S. Teige, et al., The Brookhaven National Laboratory E852 lead glass calorimeter system, Proc. of 5th Intl. Conf. On Calorimetry in HEP, eds. H.A. Gordon and D. Rueger, 161-166 (1995).

J. Dowd, Properties of the reaction $\pi^{-} p^{-}>p \eta \pi^{+} \pi^{-} \pi^{-}$at $18 \mathrm{GeV} / \mathrm{c}$, Proc. of the 5 th Intern. Conf. on Hadron Spectroscopy, Manchester, England, eds. M C. Birse, G. D. Lafferty, and J. A. McGovern, World Scientific, Singapore, (1996).

T. Adams, et al., Resonance production in the reaction $\pi^{\circ} p \rightarrow \eta \pi^{\circ} \pi^{0} \pi^{0}$, Proc. of the 5th Intl. Conf. on Hadron Spectroscopy, Manchester, England, eds. M. C. Birse, G. D. Lafferty, and J. A. McGovern, World Scientific, Singapore, 491-493 (1996).

T. Adams, et al., Design and performance of a cesium iodide detector. Nucl. Instrum. \& Meth.A368, p. 617-627 (1996).

B. B. Brabson, et al., Study of the $\pi^{\circ} \pi^{\circ}$ system in $\pi^{-} p$ interactions at $18 \mathrm{GeV} / \mathrm{c}$. Proc. of the $\mathrm{V}^{\text {th }}$ Intern. Conf. on Hadron Spectroscopy, Manchester, England, eds. M C. Birse, G. D. Lafferty, and J. A. McGovern, World Scientific, Singapore, 494-496 (1996).

N. Cason, et al., Study of the $\eta \pi, \eta \eta$, and $\eta^{\prime} \pi$ systems in $\pi^{\circ} p$ interactions at $18 \mathrm{GeV} / \mathrm{c}$. Proc. of the $\mathrm{V}^{\text {th }}$ Intern. Conf. on Hadron Spectroscopy, Manchester, England, eds. M. C. Birse, G. D. Lafferty, and J. A. McGovern, World Scientific, Singapore, 55-62 (1996).

N. Cason, et al., Study of the $\eta \pi, \eta \pi^{\circ}$, and $\pi^{\circ} p$ interactions at $28 \mathrm{GeV} / \mathrm{c}$. Proc. HADRON 95 Conf., Manchester, England, June 1995, M. C. Birse, G. D. Lafferty, and J. A. McGovern, Editors, World Scientific, Singapore, p. 5561 (1996).

N. Cason, et al, Study of $\pi^{+} \pi^{-} \pi^{-}$interactions at $18 \mathrm{GeV} / \mathrm{c}$. (Presented by Dennis Weygand for the E852 collaboration.) Proc. HADRON 95 Conf., Manchester, England, June 1995, M. C. Birse, G. D. Lafferty, and J. A. McGovern, Editors, World Scientific, Singapore, p. 241-247 (1996).

N. Cason, et al., Observation of $f 1(1285) \pi$ and $\eta^{\prime}(958) \pi$ in the reaction $\pi^{--} p \rightarrow p \eta \pi^{+} \pi^{-} \pi^{-}$at $18 \mathrm{GeV} / \mathrm{c}$.

Presented by J. P. Dowd for the E852 collaboration.) Proc. HADRON 95 Conf., Manchester, England, June 1995, M. C. Birse, G. D. Lafferty, and J. A. McGovern, Editors, World Scientific, Singapore, p. 497-499 (1996). 
J.M. LoSecco, et al., Search for exotic mesons in mp interactions at $18 \mathrm{GeV} / \mathrm{c}$. Proc. of the Intl. School of Nuclear Physics, Erice, Italy, 1995. Amand Faessler, Editors, Pergamon, p. 437-445 (1996).

Evidence for exotic meson production in the reaction $\pi^{-} \eta \pi^{*-} p$ at $18 \mathrm{GeV} / \mathrm{c}$, Phys. Rev. Lett. Vol. 79, No. 9, p. 1630-1633, Sept. 1997.

J. Dowd, Evidence for $J^{P C} 1^{-+}$exotic meson production in the eta piminus system, proc. of SLAC Summer School (1997).

A cylindrical drift chamber with azimuthal and axial position readout, Nucl. Instr. Meth., A386, p. 235-248, (1997).

A 3000 element lead glass electromagnetic calorimeter, Nucl. Instrum. Methods A387, pp 377-394 (1997).

G. S. Adams, et al., Observation of a New $J^{P C}=I^{+}$Exotic State in the reaction $\pi^{--} p \rightarrow \pi^{+} \pi^{--} \pi^{-} p$ at $18 \mathrm{GeV} / \mathrm{C}$, Phys. Rev. Lett. Vol 81, 26, pp 5760, Dec. 28, 1998.

S. U. Chung, et al., Evidence for exotic $J^{P C}=1^{++}$meson production in the reaction $\pi^{--} p \rightarrow \eta \pi^{-} p$ at $18 \mathrm{GeV} / \mathrm{c}$, Phys. Rev. D, Vol. 60, p. 092001-1-2 (1999).

S. U. Chung, Current status of exotic mesons, Nucl. Phys. B, Vol. 86 (1-3) pp. 341-346 (2000).

G. S.Adams, et al., Experimental evidence for hadroproduction of exotic mesons, Nucl. Phys. A, Vol. 680, pp. 334-340 (2001).

854 S. Ahmad, et al., A silicon multiplicity detector system for an experiment on the interaction of antiprotons with nuclei at BNL. IEEE Transaction on Nuclear Science, Vol. 39, 615-618, (1992).

S. Ahmad, et al., Antiproton nucleus interactions at 5 to $9 \mathrm{GeV} / \mathrm{c}$, invited contribution at the Second Biennial Conf. on Low Energy Antiproton Physics,Courmayer, Aosta Valley, Italy, Sept. 14-19, 1992.

857 J. Lowe, et al., The reaction $\pi^{-} p \rightarrow \pi^{0} \pi^{0} n$ near threshold and chiral symmetry breaking, Proc. of the Intl. Conf. on Particles and Nuclei, MIT, June 1990, abstract III-3.

M.D. Hasinoff, et al., The reaction $\pi^{\circ} p \rightarrow \pi^{0} \pi^{0} n$ near threshold, Bull. Am. Phys. Soc. 35, 1209 (1990).

J. Lowe, et al., $\pi^{\circ} p \rightarrow \pi^{0} \pi^{0} n$ near threshold and chiral symmetry breaking, Phys. Rev. C 44, 956(1991.

J. Lowe, et al.,The reaction $\pi^{-} p \rightarrow \pi^{0} \pi^{0} n$ near threshold and chiral symmetry breaking, $\pi N$, Newsletter No. 3 , Sept. 1991, ed. R.E. Cutkosky, G. Höhler, W. Kluge, B.M.K. Nefkens, p. 47.

J. Lowe, et al., The reaction $\pi^{-} p \rightarrow \pi^{\circ} \pi^{0} n$ near threshold and chiral symmetry breaking, Phys. Rev. C44, 956 (1991).

H. Burkhardt and J. Lowe, The global analysis of $\pi N \rightarrow \pi \pi N$ data, Phys. Rev. Lett. 67, 2622 (1991).

J. Lowe, et al., The reaction $\pi^{-} p \rightarrow \pi^{0} \pi^{0} n$ near threshold and chiral symmetry breaking, abstract contributed to the Fourth Conf. on Intersections between Particle and Nuclear Physics, Tucson AZ, May 24-29, 1991.

H. Burkhardt and J. Lowe, A global analysis of $\pi \rightarrow \pi \pi N$ data, invited talk at the Fourth Intl. Symposium on Pionnucleon Physics and the Structure of the Nucleon, Bad Honnef, Germany, September 9-13, 1991.

859 Y. Akiba, et al., Production of $\varphi$ mesons in central ${ }^{28} \mathrm{Si}+{ }^{196} \mathrm{Au}$ collisions at $14.6 \mathrm{~A} \mathrm{GeV/c}$, Phys. Rev. Lett. Vol. 76,12, pp.2021 (1996). 
863 H.H. Heckman, et al., Nuclear breakup and particle densities in $200 \mathrm{~A} \mathrm{GeV}-{ }^{16} \mathrm{O}$ interactions with emulsion nuclei, Proc. of XVIII Int. Symp. on Multiparticle Dynamics, Tashkent, USSR, Sept. 1987.

K.B. Bhalla, et al., Particle densities and nuclear breakup in ${ }^{16} \mathrm{O}$ - emulsion interactions, Proc. Int. Europhysics Conf. on High Energy Physics, Uppsala, Sweden, June 25-July 1, 1987 (Petit-Lancy, Switzerland: Eur. Phys. Soc. vol. I, p. 155, (1987).

E. Monnand, et al., Pseudorapidity densities in central ${ }^{16} \mathrm{O}$ interactions at $200 \mathrm{~A} \mathrm{GeV}$, Proc. Workshop held at Moscow, USSR, August 1987, during the 20th Int. Cosmic Ray Conf., Moscow, p. 4-10, (1988).

M.I. Adamovich, et al., Multiplicities and rapidity densities in $200 \mathrm{~A} \mathrm{GeV}{ }^{16} \mathrm{O}$ interactions with emulsion nuclei, Phys. Lett. B201, 397 (1988).

E. Friedlander, et al., A search for non-statistical fluctuations and structures in the rapidity density distribution of ${ }^{16} \mathrm{O}+\mathrm{Ag}(B R)$ and ${ }^{32} \mathrm{~S}+\mathrm{Au}$ interactions at 60 and $200 \mathrm{~A} \mathrm{GeV}$. Proc. Hadronic Matter in Collisions, Tucson, Arizona, USA, 1988, p. 210-221, Ed: P. Carruthers and J. Rafelski, World Scientific, Singapore.

S. Garpman, et al., A computer based coordinate measuring station for nuclear emulsion chambers, Nucl. Instr. Meth. A269, 134 (1988).

S. Lokanathan, et al., The EMU-01 experiment: A status report, Proc. Int. Conf. on Physics and Astro-physics of Quark Gluon Plasma, Bombay, India, Feb. 1988, p. 514- 522, Ed: B. Sinha and S. Raha, World Scientific, Singapore.

M.I. Tretyakova, et al., Central intersections of ${ }^{16} \mathrm{O}$ Nuclei with heavy nuclei of photo-emulsion at 200 GeV/Nucleon, Lebedev Reprint 244 (1988). Communication at IX Int. Seminar on High Energy Physics Problems-Relativistic Nuclear Physics and Quantum Chromodynamics, Dubna, June 1988.

R.J. Wilkes, et al., Pseudorapidity distributions and correlations in central ${ }^{16} \mathrm{O}$ intersections at $200 \mathrm{~A} \mathrm{GeV}$, Proc. 3rd Conf. on the Intersections between Particle and Nuclear Physics, Rockport, ME, USA, 14-15 May 1988. AIP Conf. Proc. 176, 1047 (1988).

M.I. Adamovich, et al., Production of helium $(Z=2)$ projectile fragments in ${ }^{16} \mathrm{O}$ interactions at $14.6,60$ and $200 \mathrm{~A}$ GeV, Phys. Rev. C. 40, 66 (1989).

M.I. Adamovich, et al., Limiting fragmentation in oxygen induced emulsion interactions at $14.6,60 \& 200 \mathrm{~A} \mathrm{GeV}$, Phys. Rev. Lett. 62, 2801 (1989).

M.I. Adamovich, et al., Scaling properties of charged particle multiplicity distribution in oxygen induced emulsion interactions at 14.6, 60 and $200 \mathrm{~A} \mathrm{GeV}$, Phys. Lett. 223B, 262 (1989).

M.I. Adamovich, et al., Charged particle spectra in oxygen induced reactions at 14.6 and $60 \mathrm{GeV} / \mathrm{Nucleon}$, High Energy Physics and Nuclear Physics in China 13, 865 (1989). (English and Chinese).

M.I. Adamovich, et al., Rapidity densities and their fluctuations in central $200 \mathrm{~A} \mathrm{GeV}{ }^{32} \mathrm{~S}$ interactions with $\mathrm{Au}$ and AG, Br nuclei, Phys. Lett. 227B, 285 (1989).

M.I. Adamovich, et al., A study of recoil protons in ultra-relativistic nucleus-nucleus collisions, Phys. Lett. 230B, 175 (1989).

S. Persson, Measurement and three-dimensional reconstruction of particle tracks in emulsion chambers, Comp. Phys. Comm. 55, 103 (1989).

E. Stenlund, et al., A search for non-statistical particle density fluctuations in ${ }^{16} \mathrm{O}+A g(B r)$ and ${ }^{32} S+A u$ interactions at $200 \mathrm{~A}$ GeV, Nucl. Phys. A498, 541c (1989). 
E. Ganssauge, et al., A Track Reconstruction Program (TRP) for evaluation of nucleus-nucleus collisions in nuclear track emulsion chambers, Comp. Phys. Comm. 55, 233 (1989).

M.I. Adamovich, et al., On the energy and mass dependence of the multiplicity in relativistic heavy-ion interactions, Modern Physics Letters A5, 169 (1990).

M.I. Adamovich, et al., Target nucleus fragmentation in ${ }^{16} \mathrm{O}+(\mathrm{Ag}, \mathrm{Br})$ interactions at $200 \mathrm{~A} \mathrm{GeV}$, Phys. Lett. 234B, 180 (1990).

M.I. Adamovich, et al., On the multiplicity fluctuations in relativistic heavy-ion collisions, Phys. Lett. 242B, 512 (1990).

M.I. Adamovich, et al., Scaled factorial moment analysis of $200 \mathrm{~A} \mathrm{GeV} \mathrm{sulphur}+$ gold interactions, Phys. Rev. Lett. 65, 412 (1990).

E. Ganssauge, et al., Contribution of the EMUO1-collaboration to the research for a quark gluon plasma (QGP), Proc. XV Int. Conf. on Particle Tracks in Solids, Marburg, FRG, Sept. 3-7, 1990.

I. Otterlund, et al., Limiting fragmentation, scaling and substructural dependence of multi-particle production in high-energy heavy-ion interactions, Physica Scripta T32, 168 (1990).

I. Otterlund, et al., Multiplicity fluctuations in high-energy heavy-ion interactions observed in high-angular resolution tracking detectors, to appear in Proc. Santa Fe Workshop on Intermittency in High-Energy Collisions. Santa Fe, NM, March 1990.

1. Otterlund, et al., Stochastic emission and non-statistical fluctuations in relativistic heavy-ion interactions, Proc. $X$ Int. Seminar on High Energy Physics Problems--Relativistic Nuclear Physics and Quantum Chromodynamics, Dubna, Sept. 1990.

E. Stenlund, et al., Non-statistical fluctuations in relativistic heavy-ion collisions, Proc. CAMP-meeting, Marburg, Germany, May 1990, p. 337.

E. Stenlund, et al., Recent results from EMUO1, Lund University Preprint LUIP 9004 (1990), Proc. HIPAGS Work-shop, Brookhaven National Laboratory, Upton, NY, March 5-7, 1990.

M.I. Tretyakova, et al., Central interactions $S+A g B r$ at $200 \mathrm{~A} \mathrm{GeV}$, Proc. X Int. Seminar on High Energy Physics Problems-Relativistic Nuclear Physics and Quantum Chromodynamics, Dubna, Sept. 1990.

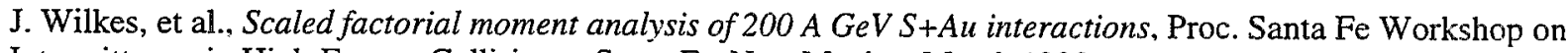
Intermittency in High-Energy Collisions. Santa Fe, New Mexico, March 1990.

R.J. Wilkes, et al., Scaled factorial moment analysis of $200 \mathrm{~A} \mathrm{GeV} \mathrm{sulfur} \mathrm{+} \mathrm{gold} \mathrm{interactions,} \mathrm{(EMU-01} \mathrm{Collabor-}$ ation), Proc. of the Santa Fe Workshop, Intermittency in High Energy Collisions, Los Alamos National Laboratory, Low Alamos, USA, March 18-21, 1990, eds. F. Cooper, R.C. Hwa, I. Sarcevic, World Scientific, p. 145 (1991).

M.I. Adamovich, et al., Stochastic emission of particles in ultra-relativistic heavy ion collisions, Modern Physics Letters A6:469 (1991).

M.I. Adamovich, et al. Stochastic emission of particles in ultra-relativistic heavy-ion collisions,(EMU-01 Collab.), High Energy Physics and Nuclear Physics in China, 15, 131 (1991).

M.I. Adamovich, et al., Energy, target, projectile and multiplicity dependence of Intermittency behavior in high-energy $O(S)$-emulsion interactions, Z Phys. C49:395 (1991). 
M.I. Adamovich, et al., On the systematic behavior of the intermittency-indices in nuclear interactions, (EMU-01 Collaboration) Phys. Lett. B, 263(3,4), 539-543 (1991).

M.I. Adamovich, et al., Stochastic emission of particles in ultra-relativistic heavy-ion collisions, (EMU-01 Collaboration), Modern Phys. Lett. A. 6(6), 469-478 (1991).

R.J. Wilkes, et al., Analysis of fluctuations and correlations in $200 * A \mathrm{GeV} A-A$ collisions, (EMU-01 Collaboration), Proc. 22nd Intl. Cosmic Ray Conf., 4:21, (Dublin, 1991).

M.I. Adamovich, et al., Energy, target, projectile and multiplicity dependencies of intermittency behavior in high energy $O($ si,S) induced interactions,(EMU-01 Collaboration), Z. Phys. C - Particles and Fields 49, 395-399 (1991).

M.I. Adamovich, et al., Multiplicities in ${ }^{16} \mathrm{O}$-induced violent heavy-ion collisions from $5 \mathrm{~A}$ to $2 \times 10^{5} \mathrm{~A} \mathrm{MeV}$, (EMU-01 Collab.), Phys. Rev. Lett. 67(10), 1201-1205 (1991).

T.C. Awes, et al., Review of recent results on particle production from EMU-01, R.J. Wilkes and the EMU-012 Collaboration, Nuclear Physics A544:153c, (1992).

V.S. Bhatia, et al., Emission characteristics of helium fragments in S-emulsion interactions at $200 \mathrm{~A}$ GeV energy. Proc. XXI Int. Symp. on Multiparticle Dynamics, Wuhan, China, Sept. 23-27, 1991, p. 623-626. (World Scientific, Singapore, 1992, Eds.: Wu Yuanfang and Liu Lianshou.)

I. Otterlund, et al., The energy dependence of particle densities and multiplicities in violent nucleus-nucleus collisions. Proc. XXI Int. Symp. on Multiparticle Dynamics, Wuhan, China, Sept. 23-27, 1991. p. 573-581. (World Scientific, Singapore, 1992, Eds.: Wu Yuanfang and Liu Lianshou).

R. J. Wilkes, et al., Review of recent results on particle production from EMU01. Proc. Quark Matter 91, Gatlinburg, Tennessee, November 11-15, 1991. Nucl. Phys. A544, 153c, (1992).

S. Garpman, et al., Linear and non-linear aspects of relativistic heavy-ion interactions from EMU01 results. Proc. 7th Int. Symp. on Very High Energy Cosmic Ray Interactions, Ann Arbor, Michigan, USA, June 21-27, (1992).

I. Otterlund, et al., EMUO1 Results from oxygen, silicon and sulphur induced violent interactions at BNL-AGS and CERN-SPS. Proc. Int. Conf. on Hadron Structure '92, Stará Lesná, Czechoslovakia, Sept. 6-11, 1992, p. $244-$ (Košice, 1992, eds: D. Bruncko and J. Urban).

M. I. Adamovich, et al., A systematic study of the energy independent behavior of the fragmentation regions in 016 EM interactions from 3.7-A-GeV to 200:A-GeV. Z. Phys. C55, 235-24, (1992).

M. I. Adamovich, et al., A systematic investigation of scaled factorial cumulant moments for nucleus-nucleus interactions, (EMU-01 Collaboration) Physical Review D47:3726 (1993).

M. I. Adamovich, et al., On the jet-like and ring-like substructure in distributions of produced particles in central heavy ion collisions at ultrarelativistic energies. J. Phys. G19:2035-2044, (1993).

E. Stenlund, et al., Pseudorapidity distributions and azimuthal substructure in heavy ion and very heavy ion collisions at energies up to $200 \mathrm{~A} \mathrm{GeV}$. Proc. 22nd Int. Symp. on Multiparticle Dynamics, Santiago de Compostella, Spain, July 13-17, 1992. p. 349-365. World Scientific, Singapore, 1993, Ed.: C.Pajares) .

I. Otterlund, et al., Gentle and violent gold interactions from the BNL AGS. Proc. Heavy Ion Physics at the AGS, HIPAGS'93, Massachusetts Institute of Technology, Cambridge, MA, January 13-15, 1993. MITLNS-2157, p. 1. (Eds. S. G. F. Stephans, S. G. Steadman and W. L. Kehoe).

I.Nystrand, et al., Rapidity density distributions in ${ }^{16} \mathrm{O},{ }^{28} \mathrm{Si}$, and ${ }^{197} \mathrm{Au}$ induced high energy heavy ion interctions, Proc. $2^{\text {nd }}$ Int. Conf. on Physics and Astrophysics of Quark-Gluon Plasma, Calcutta, India, January 19-23, 1994. p. 462 (World Scientific, Singapore, 1994), Eds.: B. Sinha, Y. P. Viyogi and S. Raha). 
E. Stenlund, et al., Violent Au-induced collisions at the BNL AGS and comparisons with expectations from particle densities and their fluctuations in collisions with lighter systems. Proc. Cracow Workshop on Multiparticle Productions - Soft-Physics and Fluctuations, Cracow, Poland, May 4-7, 1993. (World Scientific, Singapore, 1994, Eds.: A. Bialas, K. Fialkowski, K. Zalewski and R. C. Hwa.)

S. Garpman, et al., Multiparticle production in energetic nucleus-nucleus collisions, Proc. Hadron Structure 93 Int. Conf., Banska Stiavnica, Slovakia, Sept. 5-10, 1993. p. 83. (Bratislava 1993, Eds.: S. Dubnicka and A. Z. Dubnickova).

S. Vokal, et al., Interactions of $14.6 \mathrm{~A} \mathrm{GeV/c} \mathrm{silicon} \mathrm{nuclei} \mathrm{in} \mathrm{nuclear} \mathrm{emulsion,} \mathrm{Proc.} \mathrm{Hadron} \mathrm{Structure} 93$ Int. Conf., Banska Stiavnica, Slovakia, Sept. 5-10, 1993. p. 93. (Bratislava 1993, Eds.: S. Dubnicka and A. Z. Dubnickova).

J. Nystrand, et al., Rapidity density distributions in $A u+A u$ and $A u+A g$ interactions at $11.6 \mathrm{~A} \mathrm{GeV/c}$. Proc. Quark Matter '93, Borlänge, Sweden, June 20-24, 1993. Nucl. Phys. A566 (1994) 419c.

R. J. Wilkes, et al., Systematics of pseudorapidity distributions.Proc. 23rd Int. Cosmic Ray Conf., Calgary, Canada, July 1993.

V. Navotny, et al., Rapidity and azimuthal correlations in relativistic heavy-ion interactions at $4-200 \mathrm{GeV} /$ nucleon. Proc. VIII Int. Symp. on, Japan, July 24-20, p. 339, (1994).

X.Cai, et al., Fluctuations and correlations of particles produced in ultra-high-energy heavy-ion induced interactions. Proc.First Int. Symp.on Cosmic Ray Physics in Tibet (ISCRP), Lhasa,China. Aug.12-17, 1994.

E. Stenlund, et al., Aspects on Au-induced interactions with emulsion and heavy nuclei at $11.6 \mathrm{~A} \mathrm{GeV}$. Proc. Int. Conf. on Hadron Structure '94, Košice, Slovakia, p. 188, Sept. 19-23, 1994 (Košice 1994, eds.: J. Urbán and J. Vrláková)

M. I. Tretyakova, et al., Factorial moments in central Si+Ag, Brinteractions at $14.6 \mathrm{~A} \mathrm{GeV/c}$. Proc. Int. Conf. on Hadron Structure '94, Košice, Slovakia, Sept. 19-23, 1994 (Košice 1994, eds.: J. Urbán and J. Vrláková).

E. Stenlund, et al., Particle poduction in god and lead induced interactions at AGS and SPS.Proc. Quark Matter '95, Monterey, CA, USA, 9-13 January, 1995.

864 C. F. Baillie, et al., A new MCRG calculation of the critical behavior of the 3-D ising model: Preliminary results, Nucl. Phys. Proc. Suppl. 17, p. 323-327, (1990).

T. A. Armstrong, et al., Strangelets, antimatter and coalescence: First results from BNL E864, Nucl. Phys. A610, 297c, (1996).

C.A. Pruneau, et al., The E864 lead-scintillating fiber hadronic calorimeter, VI Intl. Conf. On Calorimetry in High-energy Physics, Frascati, Italy, June 1996.

J. G. Lajoie, et al., Cross sections for $K$ and antiproton production in $11.6 \mathrm{GeV} / \mathrm{c}$ per nucleon $\mathrm{A} u+P b$ collisions, Heavy Ion Physics 4, Budapest, Hungary, (1996).

K. N. Barish, et al., First search for charged strangelets with the E864 spectrometer, Heavy Ion Physics 4, 423, Budapest, Hungary, (1996).

P. Haridas, I. A. Pless, G. Van Buren, J. Tomasi, M.S.Z. Rabin. K. Barish, R. D. Majka, A 10 MHZ beam counter and a multiplicity detector for the E864 spectrometer, Nucl. Instru. and Meth. A385, 412, (1997).

T. A. Armstrong, et al., Search for exotic strange quark matter in high-energy nuclear reactions, Nucl. Phys. A625, 494, (1997). 
T. A. Armstrong, et al., Antiproton production in $11.5 \mathrm{~A}-\mathrm{GeV} / \mathrm{C} A U+P B$ nucleus-nucleus collisions, NUCLEX9709005, Sept. 1997, Phys. Rev. Lett. 79, p. 3351-3354, (1997).

K. N. Barish (for the collaboration), Strangelet searches in high-energy heavy ion collisions, NUCLEX-9711006, Nov. 1997, contributed to 6th Intl. Conf. On Hypernuclear and Strange Particle Physics (HYP 97), Upton, NY, Oct. 13-18, 1997.

T. A. Armstrong, et al., Antiproton production in $11.5 \mathrm{~A}-\mathrm{GeV} / \mathrm{C} A U+P B$ nucleus-nucleus collisions, Phys. Rev. Lett. Vol. 79, No. 18, p.3351-3354, Nov. 3, 1997.

T. A. Armstrong, et al., Search for charged strange quark matter produced in $11.5 \mathrm{~A} \mathrm{GeV/c} \mathrm{Au}+\mathrm{Pb}$ Nucleus collisions, Phys. Rev. Lett. 79, 3612, (1997).

P. Haridas, et al., A $10 \mathrm{MHZ}$ beam counter and a multiplicity detector for the E864 spectrometer, Nucl. Instr. Meth. A385, 412 (1997).

T. A. Armstrong, et al., The E864 lead-scintillating fiber hadronic calorimeter, Nucl. Instr. and Meth. A406 227 (1998).

J. Barish, et al., The E864 lead-scintillating fiber hadronic calorimeter, Nucl. Instrum. And Meth. A406 227, p.494-512 (1998).

C. A. Pruneau, et al., Recent results from experiment 864, Nucl. Phys. A638, 575c (1998).

K.N. Barish (for E864 collaboration), Strangelet searches in high energy heavy ion collisions, Nuc. Phys. A639, pp. 423c-432c (1998).

J. C. Hill, et al., A highmass trigger for the E864 experiment at the AGS accelerator, Nucl. Instr. And Meth.A421, 431 (1999).

T. A. Armstrong, et al., Mass dependence of light-nucleus production in ultrarelativistic heavy-ion collisions, Phys. Rev. Lett., Vol. 83, No. 26, p. 5431-5434, Dec. 27, 1999.

T. A. Armstrong, et al., Measurements of neutrons in $11.5 \mathrm{~A} \mathrm{GeV/C} \mathrm{Au+Pb}$ heavy-ion collisions, Phys. Rev. C., Vol. 60, pg. 064903-1-10, 1999.

J.C. Hill, et al., A high mass trigger for the E864 experiment at the AGS accelerator, Nuc. Inst. and Methods in Phys Research, Sec. A, p. 431-446, (1999).

T. A. Armstrong, et al., Search for neutral strange quark matter in high energy heavy ion collisions, Phys. Rev. C, Vol. 59, No. 4, p.R1829-1833, April, 1999.

T. A. Armstrong, et al., Antiproton production and antideuteron production limits in relativistic heavy ion collisions, Phys. Rev. C, Vol. 59, No. 5, p. 2699-2712, May, 1999.

T. A. Armstrong, et al., Search for neutral strange quark matter in high energy heavy ion collisions, Phys. Rev. C59, R1829 (1999).

T. A. Armstrong, et al., Large straw-tube tracking chambers for AGS experiment E864, Nucl. Instr. And Meth. A425, 210 (1999).

T. A. Armstrong, et al., A spectrometer for study of high mass objects in relativistic heavy ion reactions, accepted in Nucl. Instr. And Meth (1999). 
865 N. J. Baker, et al., Search for short-live neutral particles emitted in $K^{+}$decay, Phys. Rev. Lett. Vol. 59, No. 25, p. 2832-2835, Dec. 21, 1987.

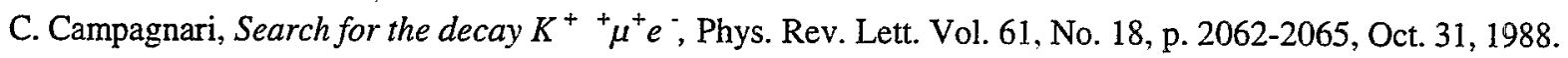

A. M. Lee, et al., Improved limited on the branching ratio of $\mathrm{K}^{+}{ }^{+} \mu^{+} e^{*}$, Phys. Rev. Lett. Vol. 64, No. 2, p 165168, Jan. 8, 1990.

D. M. Lazarus, Results/prospects in E777/851/865, 2nd Intl. Workshop on Heavy Quark Physics in Fixed Targets, U. Va., Carlottesville, VA., HQ94, Oct. 6-10,1994.

R. Appel, et al., New Measurement of the Properties of the Rare Decay $K^{+} \rightarrow \pi^{+} e+e^{+} e^{-}$, Phys. Rev. Lett. Vol. 83, No. 22, p.4482-4485, Nov. 29, 1999.

H. Ma, et al., New measurement of the rare decay $K^{+} \rightarrow \pi^{+} \mu^{+} \mu^{-}$, Phy. Rev. Lett. Vol. 84, 12, pp.2580, (2000).

R. Appel, et al., Improved limit on the rate of the decay $K^{+} \rightarrow \pi^{+} \pi^{+} e^{\cdot}$, Phys. Rev. Lett, Vol. 85, 12, pp.450, (2000).

R. Appel, et al., Search for lepton flavor violation in $K^{+}$decays into a charged pion and two leptons, Phys. Rev. lett, Vol. 85, 14, pp. 2877 (2000).

866 M. Gonin, First results with the Au-beam at the AGS, Proc.Intl. Nuclear Physics Conf., Wiesbaden, Germany, July 26-Aug. 1, 1992, Nucl. Phys. A 533, 799c-812c (1993).

H. Hamagaki (for the E866 collaboration), Semi-inclusive hadron spectra with beams of Si and Au at the AGS, BNL-49476, proc. 10th Intl. Conf. Of Ultra-Relativistic Collisions (Quark Matter 1993), Borlange, Sweden, June 20-24, 1993, Nucl. Phys. A 566, 27c-34c (1994)..

M. N. Namboodiri (for the E866 collaboration), Backward emission of protons in Au+Au collisions at 11.7 $A \cdot G e V / C$, BNL-49674, Proc. 10th Intl. Conf. of Ultra-Relativistic Collisions (Quark Matter 1993), Borlange, Sweden, June 20-24, 1993, Nucl. Phys. A 566, 443-6c (1994)..

B. Moskowitz and M. Gonin (for the E866 collaboration), Distributions of transverse energy, protons, and mesons from $\mathrm{Au}+\mathrm{Au}$ collisions at 11.6A GeV/c, BNL-49498, Proc. 13th Particles and NucleiIntl. Conf., (PANIC93), Perugia, Italy, June 28-July 2, 1993.

B. Moskowitz, et al., (for E802/E866 collaboration), Global transverse and forward energy measurements for $S i+A$ and $A u+A$ at the AGS, Proc. Heavy Ion Physics at the AGS, Jan. 13-15, 1993, Cambridge, MA, ed. G.S.F. Stephans, S.G. Steadman and W.L. Keohoe, MITLNS-2158, p. 21-9, (1993).

J. B. Costales, H.C. Britt, M.N. Namboodiri, T.C. Sangster, J.H. Thomas, and H.E. Wegner, A phoswich array for relativistic heavy ion collisions, Nucl. Instr. And Meth. A330, 183-94 (1993).

M. Gonin, et al., (for E802/E866 collaboration), Baryon distributions from meson production in Au+Au at 11.6 $A G e V / c$. First particle spectra from E866, Proc. 10th Intl. conf. on Ultra-Relativistic Nucleus-Nucleus Collisions (Quark Matter '93), June 20-24, 1993, Borlange, Sweden, Nucl. Phys. A566, 601c-4c (1994).

C. Sangster, et al., (for E802 collaboration), Target rapidity baryon distributions in ${ }^{28} \mathrm{Si}+{ }^{197} \mathrm{Au}+{ }^{197} \mathrm{Au}$ collisions at 14.6 and $11.7 \mathrm{~A}$. GeV/c., Proc. Heavy Ion Physics at the AGS, Jan. 13-15, 1993, Cambridge, MA., ed. G.S.F. Stephans, S.G. Steadman and W.L. Kehoe, MITLNS-2158, p. 61-71 (1993).

M. Gonin, O. Hansen, B.Moskowitz, F. Videbaek, H. Sorge and R. Mattiello, Comparison of experimental data to the relativistic quantum molecular dynamics model for Si+qu collisions at $14.6 \mathrm{~A} \mathrm{GeV/c}$, Phys. Rev. C 51, 310-7 (1994). 
B. Moskowkitz, et al., (for E802/E866 collaboration), Proton and produced particle distributions from $A u+A u$ collisions at 11.6 A GeV/c, Proc. 8th Mtg. Division of Particle and fields, Aug. 2-6, 1994, Albuquerque, New Mexico.

R. Debbe, S. Gushue, B. Moskowitz, J. Norris, J. Olness, F. Videbaek, In beam tests of a ring imaging Cerenkov detector with a multianode photomultiplier readout, Nucl. Instrum. Meth, A 362, 253-60 (1995).

Z. Chen, et al., (for E802 collaboration), Recent results of E866 on hadron production in $A u+A u$ reaction at $A G S$ energies, Proc. First Intl. Conf. On Frontiers of Physics, Aug. 5-9, 1995, Shantou, China.

K. Shigaki, et al., (for E802 collaboration), Study of hadron production in Au+Au collisions at $11 \mathrm{~A} \mathrm{GeV/c}$ with the AGS E866 forward spectrometer, Proc. 11th Intl. Conf. On Ultra-relativistic Nucleus-Nucleus Collisions, (quark Matter '95), Jan. 9-13, 1995, Monterey, Calif., Nucl. Phys. A 590, 519c-22c (1995).

Y. Akiba, et al., Particle production in Au+Au collisions from BNL E866, Proc. Of Quark Matter '96 -12th Intl. Conf. On Ultra-Relativistic Nucleus-Nucleus Collisions, May 20-24, 1996, Heidelberg, Germany, Nucl. Phys. A610, 139c-152c (1996).K. Kurita, et al., Azimuthal asymmetry of particle production in Au+Au collisions at 11.6 A GeV/c, Proc. of Heavy Ion Physics at the AGS (HIPAGS'96), Aug. 22-24, 1996, Detroit, Michigan.

Z. Chen, et al., Hadronic spectra in $A u+A U$ reactions at $11.6 \mathrm{~A}$ GeV/c: rapidity and $m_{t}$ distributions, Proc. of heavy Ion Physics at the AGS (HIPAGS'96), Aug. 22-24, 1996, Detroit, Michigan.

H. Sako, et al., Antiproton production in $11.7 \mathrm{AGeV/C} A u+A u$ collisions from E866, Proc. of Heavy Ion Physics at the AGS (HIPAGS'96) Aug. 22-24, 1996, Detroit, Michigan.

$\mathrm{K}$. Ashktorab, et al., Composite particle production in relativistic Au+Au collisions at AGS:first results from the E866 forward spectrometer @ 2, 4 and 10.8 A GeV, Proc. of Heavy Ion Physics at the AGS (HIPAGS'96), Aug. 22-24, 1996, Detroit, Michigan.

L. Ahle, et al., Baryon emission at target rapidities in $S i+A 1, C u$, Au collisions at $14.6 \mathrm{~A}$ GeV/c and $\mathrm{Au}+\mathrm{Au}$ collisions at 11.7A GeV/c, Phys. Rev. C55 (5), 2604-2614 (1997).

R.Debbe, C. Muentz, J.B. Cummings, A high resolution quart Cerenkov detector for relativistic heavy ion beams, Nucl. Instrum. Meth. A403, p.256-262 (1998).

L. Ahle, et al., Particle production at high baryon density in central Au+Au reactions at $11.6 \mathrm{~A}$ GeV/c, Phys. Rev. C, Vol. 57, No. 2 (1998).

L. Ahle, et al., Kaon production I Au+Au collisions at 11.6A GeV/c, Phy. Rev. C, Vol. 58, 6, pp. 58 (1998).

L. Ahle, et al., Antiproton production in Au+Au collisions at 11.7A GeV/c, Phys. Rev. Lett, Vol. 81, 13, pp.2650, (1998).

L. Ahle, et al., Proton, deuteron, and triton emission at target rapidity in Au+Au collisions at $10.20 \mathrm{~A} \mathrm{GeV:}$ spectra and directed flow, Phys. Rev. C, Vol. 57, 3, pp.1416, (1998).

L. Ahle, et al., $A u+A u$ reactions at the AGS: Experiments E866 and E917, Nucl. Physics A., Vol. 638, pp. 57-68 (1998).

L. Ahle, et al., Proton and deuteron production I Au + Au reactions at $11.6 \mathrm{~A} \mathrm{GeV/c}$, Phy. Rev. C., Vol. 60 , 064901, pp. 60 (1999).

L. Ahle, et al., Simultaneous multiplicity and forwardenergy characterizaton of particle spectra in $\mathrm{Au}+\mathrm{Au}$ collisions at 11.6A GeV/c, Phy. Rev. C., Vol. 59, 4, pp. 2173, (1999). 
L. Ahle, et al., Centrality dependence of kaon yields in Si+A and Au+Au collisions at relativistic energies, Phy. Rev. C, Vol. 60, 044904, pp. 60, (1999).

\section{KLMM Collaboration}

W. Wolter, et al., Evidence for a critical temperature in excited target nuclei due to high energy nuclear interactions, 23rd ICRC Calgary, Canada, vol. 4, p. 5-8 (1993).

R. Holynski, et al., Comparison of particle production in nucleus-nucleus collisions with predictions of the Venus Monte Carlo model, 23rd ICRC, Calgary, Canada, vol. 4, p. 9-12 (1993).

R. Holynski, et al., Evidence for a nuclear phase transition in target nuclei after relativistic nuclear inter-actions, A. Dabrowska, Zeitschrift fur Physik, Parts. and Fields, 59(3), 399-403 (1993).

K. Sengupta, et al., Multifractal analysis of nucleus-nucleus interactions, Phys. Rev. C 48, 3174-3181 (1993).

M. L. Cherry, et al., Interactions of 10.6 GeV/nucleon gold nuclei in nuclear emulsions, Zeitschrift fur Physik, C62, 25, (1994).

M. L. Cherry, et al., Interactions of $10.6 \mathrm{GeV} / \mathrm{n}$ gold nuclei with light and heavy target nuclei in nuclear emulsions, Zeitschrift fur Physik, C63, 549 (1994).

M. L. Cherry, et al., Fragmentation and multifragmentation of $10.6 \mathrm{~A} \mathrm{GeV}$ gold nuclei, Phys. Rev. C52, 26522662 (1995).

M. L. Cherry, et al., Intermittency in ${ }^{197}$ Au fragmentation, Phys. Rev. C53, 1532 (1996).

M. L. Cherry, et al., Transverse momenta of helium fragments in gold fragmentation at $10.6 \mathrm{GeV} /$ nucleon, Zeitschrift fur Physik, 73, 449 (1997).

B. Wilczynska, eta l., Transverse monenta of helium fragments from gold projectiles in selected classes of nucleus-nucleus collisions, 25th ICRC, Durban, Vol. 6, p. 13-15. (1997).

\section{UHIC Collaboration}

C. Jake Waddington, Some preliminary results from the new $10.6 \mathrm{GeV} /$ nucleon gold beam at Brookhaven, Inter. J. of Mod. Phys. E, Vol. 2, No. 4, 739-766, (1993).

L. Y. Geer, J. Klarmann, B. S. Nilsen, C. J. Waddington, W. R. Binns, J. R. Cummings and T. L. Garrard, Energy dependence of the fragmentation of UH-nuclei, 23rd ICRC, Calgary, Canada, vol. 2, p. 191-194, (1993).

B. S. Nilsen, C. J. Waddington, W. R. Binns, J. R. Cummings, T. L. Garrard, L. Y. Geer, and J. Klarmann, Charge-pickup by heavy relativistic nuclei, Phys. Rev. C50, 1065-1076, (1994).

J. Waddington, W. R. Binns, J. R. Cummings, T. L. Garrard, B. W. Gauld, L. Y. Geer, J. Klarmann, and B. S. Nilsen, Interactions of $10.6 \mathrm{GeV} / \mathrm{n}$ gold nuclei in targets from ${ }_{1} \mathrm{H}$ to ${ }_{82} \mathrm{~Pb}, \mathrm{C}$. Nucl. Phys. $\Lambda 566,427 \mathrm{c}-430 \mathrm{c},(1994)$.

L. Y. Geer, J. Klarmann, B. S. Nilsen, C. J. Waddington, W. R. Binns, J. R. Cummings and T. L. Garrard, The charge-changing fragmentation of 10.6 GeV/nucleon ${ }^{197}$ Au nuclei, Phys. Rev. C52, 334-345, (1995).

C. J. Waddington, W. R. Binns, J. R. Cummings, T. L. Garrard, L. Y Geer, J. Klarmann and B. S. Nilsen, Nuclear parameters needed for interpretation of observed fluxes of UH-cosmic ray nuclei, Adv. Space Res., 15, No. 6, 3948, (1995).

C. J. Waddington, The propagation of UH cosmic ray nuclei using energy dependent cross-sections, Astrophys. J., $470,1218-1226,(1996)$. 
C. J. Waddington, J. R. Cummings, T. Garrard, P. Hink and B. S. Nilsen, Propagation of the heaviest UH cosmic ray nuclei, 25th ICRC, Durban, Vol. 4, p. 345-348, (1997).

C. J. Waddington, Can source abundances be reliably deduced from measured cosmic ray abundances?, 25th ICRC, Durban, Vol. 4, p.341-344, (1997).

871 A. P. Heinsaon, et al., Measurement of the branching ratio for the rare decay $\mathrm{K}_{\mathrm{L}}^{\circ} \rightarrow \mu^{+} \mu^{-}$, Phys. Rev. D, 51, p.985-1011, (1995).

D. Ambrose, et al., (for the E871 Collaboration), First ibservation of the rare decay mode $\mathrm{K}_{\mathrm{L}}^{0} \rightarrow e^{+} e^{-}$, UTEXASHEP-98-14, Odt. 1998, Phys. Rev. Lett. 81, No. 20, p. 4309-4312, Nov. 16, 1998.

D.Ambrose, et al., New limit on muon and electron lepton number violation from $\mathrm{K}_{\mathrm{L}}^{\mathrm{o}} \rightarrow \mu^{ \pm} e^{\mp}$ decay, Phys. Rev. Lett., 81, No. 26, p.5734-5737, Dec. 28, 1998.

J.Belz, et al., A compact beam stop for a rare kaon decay experiment, Nuc. Inst. Methods in Physcs Research A 428, p.239-262 (1999).

D. Ambrose, et al., Improved branching ratio measurement for the decay $\mathrm{K}_{\mathrm{L}}^{0} \rightarrow \mu^{+} \mu^{-}$, phys. Rev. Lett., Vol. 84, No. 7, p.1389-1392, Feb. 14, 2000.

874 C. Kormanyos, et al. Nuclear response to quasifree $K^{+}$scattering, Spring Meeting- American Physical Society, April 12-15, 1993.

C. Kormanyos, et al., Nuclear quasi elastic $K^{+}$scattering, Phys. Rev. Lett. 71, 2571 (1993).

C. Kormanyos, et al., Quasi elastic $K^{+}$scattering, Phys. Rev. C51 669 (1995).

C. Kormanyos and R. J. Peterson, Quasi elastic $K^{+}$nucleus scattering and 'swollen nucleons', Nucl. Phys . A 585, 113 (1995)."

875 A. Mukhopadhyay, P. L. Jain and G. Singh, Multiplicity distributions in high-energy heavy-ion collisions, Nuova Cim. 106A, 967-978 (1993).

P. L. Jain, G. Singh and A. Mukhopadhyay, Fractal analysis of projectile fragments in nuclear collisions at 1-2A $\mathrm{GeV}$, Nucl. Phys. A561, 651-659 (1993).

A. Mukhopadhyay, P. L. Jain and G. Singh, Multiplicity distributions in forward and backward hemi-spheres at high energy collisions, Nuova Cim. A106, 793-811 (1993).

A. Mukhopadhyay, G. Singh and P. L. Jain, J., Cluster formation in high energy collisions, Phys. G, 19, 1137$1142(1993)$.

P. L. Jain, G. Singh and A. Mukhopadhyay, Factoral moments and multifractal analysis at relativistic energies, Phys. Rev. C48, R517-R521 (1993).

G. Singh, A. Mukhopadhyay and P. L. Jain, Z., Intermittency and fractals in nuclear collisions at 1.52A GeV, Phys. A345, 305 (1993).

P. L. Jain and G. Singh, Multifragment disintegration of $238 U$ at IA GeV, Phys. Rev. C47, 2382-2385 (1993).

P.L. Jain, A. Mukhopadhyay and G. Singh, Z., Factoral moments and short range correlational relativistic energies, Phys. C58, 1 (1993). 
M. Golde, G. Singh, P. L. Jain, and A. Mukhopadhyay, Z., Characteristics of He-nucleus interctions at relativistic energy, Phys. A344, 291 (1993).

G. Singh, P. L. Jain and M. S. El-Nagdy, Intermittency analysis in nuclear multifragmentation, Europhys. Lett. 21, 527 (1993).

P. L. Jain, G. Singh, A. Mukhopadhyay, Intermittent behavior of nuclear multigragments, Phys. Rev. C47, 342 (1993).

A. Mukhopadhyay, P. L. Jain and G. Singh, Entropy and fractal characteristics of multiplocity production at relativistic heavy ion interactions, Phys. Rev. C47, 410 (1993).

G. Singh and P. L. Jain, Multifractal analysis of 197Au-emulsion collisions at 10.6A GeV, Phys. Rev. C (1994).

P. L. Jain, G. Singh, and A. Mukhopadhyay, Nuclear collective flow in ${ }^{197}$ Au-emulsion interaction at $10.6 \mathrm{~A} \mathrm{GeV}$. Phys. Rev. Letts. 74, 1534 (1995).

P. L. Jain and G. Singh. Characteristics of charged particle multiplicities distribution in relativistic heavy-ion interactions. Phys. Rev. C (1996).

P. L. Jain and G. Singh. Liquid-gas coexistence in ${ }^{208} \mathrm{~Pb}$-emulsion interactions at 160A GeV. Phys. Lett. B (1996).

G. Singh and P. L. Jain., Target and projectile fragmentations in ${ }^{208} \mathrm{~Pb}$-Emulsion collisions at $160 \mathrm{~A} \mathrm{GeV}$, Phys. Rev. C (1996).

877 J. Barrette, et al., Transverse energy production in reactions with $11.4 \mathrm{~A} \mathrm{GeV/c} \mathrm{Au}$ and $14.6 \mathrm{~A} \mathrm{GeV/c} \mathrm{Si,} \mathrm{the}$ E814/E877 collaboration, Phys. Rev. Lett. 70, 2996, (1993).

P. Braun-Munzinger, et al., (for E877 collaboration), Compression, expansion, and freezeout in nucleus-nucleus collisions at the AGS, in Bodrum 1993, Proc., Hot and dense nuclear matter, 419-426.

J.Barrette, et al., (for E877 collaboration), Transverse energy production with SI and AU beams at AGS energy: towards hot and dense hadronic matter, Nucl. Phys. A566, pp 411c-414c, (1994).

J. P. Wessels and Y. C. Zhang, the E877 collaboration, Is there flow at the AGS?, Proc. 10th Winter Work-shop on Nuclear Dynamics, Snowbird, Utah, Jan. 1994, W. Bauer, editor, World Scientific, Singapore, p. 228, (1994).

J. Barrette, et al., Observations of anisotropic event shapes and transverse flow in $A u+A u$ collisions at $A G S$ energy, the E877 collaboration, Phys. Rev. Lett. 73, 2532 (1994).

J. Barrette, et al., Charged particle pseudorapidity distributions in $\mathrm{Au}+\mathrm{Al}, \mathrm{Cu}, \mathrm{Au}$, and $U$ collisions at $10.8 \mathrm{~A}$ GeV/c, the E877 collaboration, Phys. Rev. C651, 3309 (1995).

J. Barrette, et al., the E877 collaboration, Directed flow and particle production in $A u+A u$ collisions from experiment 877 at the AGS, Nucl. Phys. A590, pp 259c-270, (1995).

Y. C. Zhang and J. P. Wessels, the E877 collaboration, Energy flow and particle spectra with respect to the reaction plane for Au+Au collisions at AGS energies, Nucl. Phys. A590, pp 557c-650c, (1995).

D. Miskowiec, the E877 collaboration, Pion-Pion correlations in Au+Au collisions at AGS energy, Nucl. Phys. A590, pp 605c-608c, (1995).

Sergei Voloshin, the E877 collaboration, $d N_{c h} / d$ distributions in $\mathrm{Au}+\mathrm{Al}, \mathrm{Cu}, \mathrm{Au}$, and $\mathrm{U}$ collisions at $10.8 \mathrm{~A} \mathrm{GeV/c}$ and $E_{t}$ per charge particle, Nucl. Phys. A590, pp 605c-608c, (1995). 
R. Lacasse, et al., (for the E877 collaboration) Hadron yields and spectra in $A u+A U$ collisions at the AGS, NUCLEX-9609001, May 1996, 12pp, Presented at 12th Intl. Conf. Ultra-Relativistic Nucleus-Nucleus Collisions (quark Matter 96), Heidelberg, Germany, May 20-24, 1996, Nucl. Phys., A610,pp 153c-164c, (1996).

J. Barrette, et al., (for E877 collaboration), Two pion correlations in $A U+A U$ collisions at $10.8 \mathrm{GeV} / \mathrm{c}$ per nucleon, PHYSICS-9702008, Feb. 1997 5pp, Phys. Rev. Lett 78, pp 2916-2919, (1997).

J. Barrette, et al., (for E877 collaboration), Energy and charged particle flow in a 10.8-A/GeV/c $A U+A U$ collisions, NUCLEX-9610006, Oct. 1996 12pp, Phys. Rev. C55, pp 1420-1430, 1997.

J. Barrette, et al., (for E877 collaboration), Proton and pion production relative to the reaction plane in $A U+A U$ collisions at AGS energies, HD-PY-97-07, July 1997.

878 M. J. Bennett, et al., Antiproton distributions in Au+nucleus collisions, Phys. Rev. C, Vol. 56, 3, pp. 1521, (1997).

M. J. Bennett, et al., Light nuclei production in relativistic Au+nucleus collisions, Phys. Rev. C, Vol. 58, 2, pp. $1155,(1998)$.

880 T. Roser, Properties of partially excited siberian snake, in high energy spin Physics: 8th Intl. Symposium, ed. K.J. Heller, Minneapolis, MN, 988, AIP Conf. Proc. No. 187 (AIP, New York, 1989), p.1442.

H. Huang, et al., The partial siberian snake experiment at the Brookhaven AGS, Proc. of the third European Particle Accelerator Conf., p. 729 (1992).

T. Roser, Partial siberian snake test at the Brookhaven AGS, in high energy spin physics, 10th Intl. Symposium, ed. T. Hasegawa, et al., Nagoya, Japan, p. 429 (1992).

H. Huang, et al., Preservation of proton polarization by a partial siberian snake, Physical Review Letters 73 , 2982 (1994).

H. Huang, et al., Partial siberian snake experiment at the AGS, in high energy spin physics: 11 th Intl. Symposium, ed. K. J. Heller and K. J. Smith, Bloomington, 1994, AIP Conf. Proc. No.343 (AIP, New York, 1995), p. 90.

D. G. Underwood, A review of high energy polarimetry, with a view toward RHIC, in high energy spin physics, 11 th Intl. Symposium, ed. K. J. Heller and S. O. Smith, Bloomington, 1994, AIP Conf. Proc. No. 343, p. 113 (1995).

T. Roser, Polarized proton beams, Proc. of the 1995 IEEE Particle Accelerator Conf. and Intl. Conf. on HighEnergy Accelerators, 3154 (1996).

H. Huang, et al., Polarized proton experiment in the AGS with a partial snake, proc. of 12 th Intl. Symposium on High Energy Spin Physics, Amsterdam, 528-530, Sept. 1996.

H. Huang, et al., Overcoming weak intrinsic depolarizing resonances with energy jump, 1997 IEEE Particle Accel. Conf., and Intl. Conf. on High Energy Accelerators, Vancouver, Canada, May, 1997.

H. Huang, T. Roser, A. Luccio, Spin tracking study in the AGS, 1997 IEEE Particle Accel. Conf. and Intl. Conf. on High-Energy Accelerators, Vancouver, May 1997.

M. Bai, et al., Overcoming intrinsic spin resonance by using an AC dipole, 1997 IEEE Particle Accel. Conf. and Intl. Conf. on High Energy Accelerators, Vancouver, Canada, May 1997.

M. Bai, et al., Overcoming the intrinsic spin resonance using resonance island created by RF dipole, Phys. Rev. 80,4673 (1998).

M. Bai, et al., Experimental test of coherent betatron resonance excitations, Physical Review E, 5 (1997). 
H. Huang, et al., Polarized proton beam in the AGS, 13th Intl. Symposium on High Energy Spin Physics, Protvino, Russia, (1998).

T. Roser, Acceleration of polarized proton beams, 13th Intl. Symposium on High Energy Spin Physics, Protvino, Russia, (1998).

M. Bai, et al., Overcoming intrinsic SPIM resonances with an If dipole", Phys. Rev. Lett, 80, p.4673 (1998).

T. Roser, Acceleration of polarized protons tohigh energy, Proc. 1999 IEEE Particle Accelerator Conf., New York, N.Y., Mar. 1999, Vol 1, pp. 26-30 (1999).

M.Bai, Beam manipulation with an if dipole, Proc. 1999 IEEE Particle Accelerator Conf., New York, N.Y., Mar. 1999, Vol. 1 pp 387-391 (1999).

881 C. Landberg, et al., Test of the OZI rule in hadroproduction of and $K^{+} K$, Phys. Rev. D53, p. 2839-2842 (1996).

882A Y. D. He and P. B. Price, Measurement of cross section for charge pickup by $11.4 \mathrm{~A} \mathrm{GeV} \mathrm{gold} \mathrm{ions,} \mathrm{Phys.} \mathrm{Lett.} \mathrm{B}$ 298, 50-53 (1993).

P. B. Price and Y. D. He, Interactions of $11.4 \mathrm{~A} \mathrm{GeV}{ }^{197} \mathrm{Au}$ in various targets, Proc. of $23^{\text {rd }}$ Intl. Cosmic Ray Conf., Calgary, 2, 199-202 (1993).

A. J. Westphal and Y. D. He, Measurement of cross-sections for electron capture and stripping by highly relativistic ions, Phys. Rev. Lett. 71, 1160-1163 (1993).

Y. D. He and P. B. Price, First measurement of charge changing cross sections for $11.4 \mathrm{~A} \mathrm{GeV}{ }^{197} \mathrm{Au}$ in various targets, Nucl. Phys. A 566, 363c-366c (1994).

Y. D. He and P. B. Price, Nuclear and electromagnetic fragmentation of 2.25-TeV ${ }^{197}$ Au nuclei, Z. Phys. A 348 , 105-109 (1994).

Y. D. He, A. J. Westphal, and P. B. Price, Response of the BP-1 phosphate glass detector to relativistic heavy ions, Nucl. Instr. Meth. B 84, 67-76 (1994)

A. J. Westphal, Y. D. He, and P. Wojdowski, Apparent binary microscopic response to relativistic ions of a large class of track-etch detectors, Nucl. Instr. Meth. B 86, 317-324 (1994).

M. Drndic, Y. D. He, P. B. Price, D. P. Snowden-Ifft, and A. J. Westphal, Atomic-force-microscopic study of etched nuclear tracks at extremely short distance scale, Nucl.Instr. Meth. B 93, 52-56 (1994).

Y. D. He and M. Solarz, Sensitivity of BP-I glass detectors etched in methanesulfonic acid, Nucl. Instr. Meth. B 94, 113-118 (1994).

882B Y.D. He and P. B. Price, Search for abnormal-nucleus production in heavy-ion collisions, Phys. Rev. C 48, 647-650 (1993).

Y. D. He and P. B. Price, Search for dirac magnetic monopole production in high energy heavy ion collisions, Proc. of $24^{\text {th }}$ Intl. Cosmic Ray Conf., Rome, 1, 845-848 (1995).

Y. D. He and P. B. Price, Production and detection of hyperfragments in high energy nucleus-nucleus collisions, Proc. of 24 $4^{\text {th }}$ Intl. Cosmic Ray Conf., Rome, 1, 64-67 (1995).

Y. D. He and P. B. Price, Electromagnetic production of hyperfragments in ultrarelativistic heavy-ion collisions, Nucl. Phys. A 585, 363c-364c (1995). 
Y. D. He and P. B. Price, Production and detection of hyperfragments in high energy nucleus-nucleus collisions Proc. of $24^{\text {th }}$ Intl. Cosmic Ray Conf., Rome, 1, 64-67 (1995).

Y. D. He and P. B. Price, Search for dirac magnetic monopole production in high energy heavy ion collisions, Proc. of $24^{\text {th }}$ Intl. Cosmic Ray Conf., Rome, 1, 845-848 (1995).

883 S. E. Hirzebruch, G. Rusch, E. Winkel, and W. Heinrich, Response of BP-1 to ${ }^{197}$ Au heavy ions at 11.3 GeV/nucleon, NIM B74, 519-522, (1993).

W. Heinrich, E. Winkel, G. Rusch, J. Dreute, and B. Wiegel, Multifragmentation experiments using plastic nuclear track detectors, Proc. Int. Workshop XXII, Hirschegg, p. 30 (1994).

W. Heinrich, E. Becker, J. Dreute, S. E. Hirzebruch, G. Hüntrop, M. Kurth, H. Röcher, G. Rusch, M. Schmitz, T. Streibel and E. Winkel, High energy heavy ion interactions studied with SSNTDs, Radiation Measurements, Vol. 25, Nos. 1-4, p. 203-218, (1995).

885 L. Lee, et al., A multicell target for lambda lambda hypernuclei searches, by BNL E885 Collaboration, TRI-PP94-87, Oct. 1994. 2pp, presented at Intl. Conf. on Hypernuclear and Strange Particle Physics (HYP94), Vancouver, Canada, July 4-8,1994. Published in Nucl. Phys. A585:339c-340c, (1995) (Bassalleck first author).

K. Yamamoto (for the 885 collaboration), $H$-dibaryon search via the $\left(K, K^{+}\right)$reaction using a diamond target, Proc. of Intl. Conf. On Hypernuclei and Strange Particles, Nucl. Phys. A639, p. 371-374 (1998).

M. May, Search for nuclei containing two strange quarks, Proc. of Intl. Conf. on Hypenuclei and Strange Particles, Nucl. Phys. A639, p.363-370 (1998).

M. Landry, et al., Performance of micro strip gas chambers in BNL-E885: a search for A1-hypernuclei, Nucl. Inst. Meth A, Vol. 421,1-2, pp 31-42, (1998).

T. Fukuda, et al., Cascade hypernuclei I the $\left(K, K^{+}\right)$reaction on ${ }^{12} C$, Physical Review C, Vol. 58, No. 2, p. 1306 1309 (1998).

P. Khaustov, et al., Evidence of hypernuclear production in the ${ }^{12} C\left(K . K^{+}\right)^{12}$ Be reaction, Phys. Rev. C, Vol. 61 , pp. 054603-1, (2000).

.P. Khaustov, et al., Search for double $\Lambda$ hypernuclei formation via $\left(\Xi^{-12} C\right)_{\text {atom }} \rightarrow \frac{12}{\Lambda \Lambda} B+n$, Physics Lett. B, 478 , pp. 027601-1,(2000).

J. Yamamoto, et al., Search for double- $A$ hypernuclei and the $H$-dibaryon in the $\left(K, K^{+}\right)$reaction on ${ }^{12} C$, Phys. Lett. B, 478, pp. $401-407$ (2000).

M. R.Landry, Microstrip gas detectors and a search for $\equiv$ hypernuclei, Ph.D. thesis, U. Manitoba, Winnipeg, Manitoba Canada, April 2000.

K.Yamamoto, Search for the h-dibaryon via the $\left(K, K^{+}\right)$reaction on ${ }^{12} C$, Ph.d. dissertation, Kyoto U., Kyoto, Japan, KUNX 1669, NH00D03, May 2000.

P. Khaustov, et al., Production of doubly-strange systems in the $\left(K, K^{+}\right)$reaction in E885 at BNL, Nucl. Pys. A., Vol. 663-664, pp. 485-488, (2000).

886 G.E. Diebold, et al., Production of $\pi^{ \pm}, K^{ \pm}, p$, and $\bar{p}$ in relativistic $A u+P t, S i+P t$, and $p+P t$ collisions, Phys. Rev. C, 48, 2984 (1993). 
N. Saito, et al., Composite particle production in relativistic $A u+P t, S i+P t$, and $p+P t$ collisions, Phys. Rev. C49, 3211 (1.994).

A. Rusek et, al., Search for $H$ dibaryon-nucleus bound states in relativistic Au+Pt collisions. PRC C5, 1580 (1995).

A. Rusek, et al., Search for strangelets and other rare objects in Au +Pt collisions at the AGS using a fixed angle focusing spectrometer. Proc. of Intl. Conf. on Hypernuclear and Strange Particle Physics, Vancouver, Canada, July 1994. Nuc. Phys. A 585, 59C (1995).

A. Rusek, $A$ search for strangelets and other rare objects in relativistic $A u+P t$ collisions. Dissertation submitted in partial fulfillment of the Requirements for the Degree of Ph.D. in Physics. The University of New Mexico, Albuquerque, NM, December 1995.

A. Rusek, et. al., Search for strangelets and other rare objects in Au +Pt collisions at AGS using a fixed-angle focusing spectrometer, Nucl. Phys. A585 59-62 (1995).

A. Rusek, et al., (for E886 collaboration), Search for $\mathrm{H}$ dibaryon nucleus bound states in relativistic $A u+P t$ collisions, Phys. Rev. C52, p. 1580-1583, (1995).

A. Rusek, et al., (for E886 collaboration), Strangelet search and light nucleus production in relativistic $S i+P t$ and $\mathrm{Au}+$ Pt collisions, Phys. Rev. C54, p 15-19, (1996).

887 R. Sawafta, et al., Do narrow sigma hypernuclear states exist? AGS proposal, BNL-Proposal-887, Jan. 1992, Nucl. Phys. A585, p. 103c-108c, (1995).

R. I. Sawafta (for E887 and E905 collaboration), Recent experimental results in sima hypernuclei, Nucl. Phys. A639, p. 103-110 (1998).

S.Bart, et al., Shyperons in the nucleus, Phys. Rev. Lett. Vol. 83, 25, pp. 5338, (1999).

888 J. Belz, et al., Search for diffractive dissociation of long-lived H dibaryon, Phys. Rev. D, R3487-91 (1996) and PRINCETON/HEP/95-11 (1995).

J. Belz, et al., Search for the Weak decay of an H dibaryon, Phys Rev. Letts., 76, 3277-80 (1996) and PRINCETON/HEP/95-12, UTEXAS-HEP-95-19 (1995).

M. May (for E88 collaboration), Search for the presence of $H$ particles in a neutral beam, Nucl. Phys. A., Vol. 585, pp. 97-102, (1995).

J. Belz, et al., Addendum to Search for the weak decay of an $H$ dibaryon. Phys. Rev. C., Vol. 56, 2, pp. 1164, (1997).

890 M. Clajus, et al., Eta production in pion interactions with protons and deuterons, Proc. 6th Intl. Symposium on Meson-Nucleon Physics and the Structure of the Nucleon, Blaubeuren, Germany, July 10-14, 1995, Vol. II, eds. G. J. Wagner, R. Bilger and T. Hehl.

A. Marusi, A new test of charge symmetry in eta production on deuterium, Ph.D. dissertation, U. of Zagreb, (1996).

W. B. Tippens, et al., Measurement of charge symmetry breaking by the comparison of $\pi^{+} d \rightarrow p p \eta$ with $\pi^{-} d \rightarrow \eta$, Phys. Rev. D., Vol. 63, pp 052001-1, (2001).

891 S. Ahmad, et al., Lambda production by $11.6 \mathrm{~A} \mathrm{GeV/c} \mathrm{Au} \mathrm{beam} \mathrm{on} \mathrm{Au} \mathrm{target,} \mathrm{Physics} \mathrm{Letters} \mathrm{B332,} \mathrm{p.35-39,}$ (1996). 
A. C. Saulys, et al, (for the E891 collaboration) Lambda production in Au-Au collisions at the AGS, Abstract PANIC96 Conf., May 22-28, 1996.

A. C. Saulys, et al., Production in Au-Au collisions at the AGS, presented at the PANIC 96 Conf., May 22-28, 1996.

892 N. Claytor, A. Belkacem, T. Dinneen, B.Feinberg, H. Gould, Ionization of Au $u^{78+}$ and electron capature by $A u^{79+}$ at 10.8 GeV/nucleon, Phys. Rev. A55, R842 (1997).

A. Belkacem, N. Claytor, T. Dinneen, B. Feinberg, H. Gould, Electron capture from pair production by $\mathrm{Au}^{79+}$ at $10.8 \mathrm{GeV} /$ nucleon, Phys. Rev. A 58, 1253 (1998).

893 A. J. Keane, D. O'Sullian, A. Thompson, L. Drury and K.-P. Wenzel, The charge spectrum of ultra-heavy nuclei, including actinides, in the cosmic radiation, Adv. Space Res., vol. 19, p. 739-742, (1997).

A. J. Keane, Measurement of the charge spectrum of ultra-heavy galactic cosmic rays with $Z>70$, $\mathrm{PhD}$ Thesis, University College, Dublin, Natl. University of Ireland, (1997).

A. J. Keane, A. Thompson, D. O'Sullivan, L. O'C. Drury and K.-P. Wenzel, A charge spectrum of ultra-heavy cosmic ray nuclei, including actinides, detected on the LDEF, Proc. 25th Intl. Cosmic Ray Conf., Durban, Vol 3 , p. 361-364, (1997).

895 D. Best, et al., First results on 4 production between $2.8 \mathrm{~A} \mathrm{GeV}$, fom $\mathrm{E} 895$ proc. of strangeness in quark mater, Santorini, Greece, April 1997, J.Phys. G23, 1873 (1997).

$\mathrm{H}$. Liu, et al., Collective flow in $\mathrm{Au}+\mathrm{Au}$ collisions in $2.8 \mathrm{GeV}$ energy range, Proc. of Nucleus-nucleus collisions, Gatlingburg, TN, Nuc. Phys. A638, 451c (1998).

$\mathrm{H}$. Liu, et al., Collective flow and particle spectra in relativistic heavy-ion collisions, Proc. $6^{\text {th }}$ Intl. Conf. On Nucleus-Nucleus Collisons, Gatlinburg, TN, June 1997, eds. M.Thoennessen, F. Bertrand, J. Garrett and C. Gelbke, Nucl. Phys. A630, 549c (1998).

H. Liu, et al.,, Collective flow in Au + Au collisions between 2-8A GeV at the AGS, Proc. of Quark Matter '97, Tsukuba, Japan, Dec. 1997, eds. T. Hatsuda,Y. Miake, S. Nagamiya, K.Yagi, Nucl. Phys. A638, 451c (1998).

P. Chung, et al., Neutral strange particle proeduction and flow at AGS energies, Proc. of Intl. Symposium on Strangeness in Quark Matter, Padua, Italy, 1998, ed. M. Morando, J. Phys. G25, 255 (1999).

M. A. Lisa, et al., The E895 pi correlation analysis-a status report, Advances in Nuclear Dynamics 4 (Proc. of $14^{\text {th }}$ Winder worksho on Nuclear Dynamics, Snowbird, Utah, Feb. 1998), p. 183, eds. W Bauer and H-G Ritter (Plenum, New York), 1998.

M.A. Lisa, et al., An HBT excitation function at the AGS, Proc. of $2^{\text {nd }}$ Catania Relativisitic Ion Sudies (CRIS '98), Acicastello, Italy, June 1998, eds. S. Costa, S. Albergo, A. Insolia and C. Tuve (World Scicntific, Singapore, 1999) p. 357.

G. Rai for E895 collaboration, Directed and elliptical flow in $0.25-8 \mathrm{~A} \mathrm{GeV} \mathrm{Au}+$ Au collisions, RHIC Winter Workshop at LBNL, Berkeley, Calif., Jan. 1999.

S. Y. Panitkin, et al., Beam energy depenence of two-proton correlations at the AGS, Proc. $15^{\text {th }}$ Winder Workshop on Nuclear Dynamics, Park City, Utah, Jan. 1999, (Kluwer Academic Press), pp. 271 (1999)

M.A. Lisa, et al., The bombarding energy dependence of pi interferometry at the AGS, Proc. $15^{\text {th }}$ Winder workshop on Nuclear Dynamics, park City, Utah, Jan. 199, (Kluwer Academic press,)pp. 147 (1999). 
C. Pinkenburg, et al., Elliptic flow as a probe for the equation of state of high density nuclear matter, proc. of Relativistic heavy ion minisymposium at the centennial mtg. of the American Physical Society, Atlanta, GA., Mar. 1999 (World Scientific, Signapore,) pp. 78 (1999).

$\mathrm{R}$. Witt, et al., Composite fragment production in $\mathrm{Au}+\mathrm{Au}$ collisions between $2 \mathrm{~A} \mathrm{GeV}$ and $8 \mathrm{~A} \mathrm{GeV}$, Proc. of Relativistic heavy ion minisymposium at the centennial mtg. of the American Physical Society, Atlanta, GA., Mar. 1999 (World Scientific, Signapore) pp. 297 (1999).

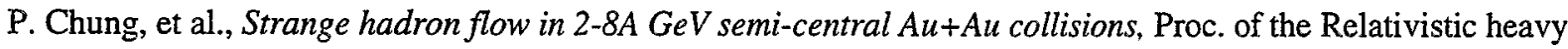
ion minisymposium at the centennial mtg. of the American Physical Society, Atlanta, GA., Mar. 1999 (World Scientific, Signapore), pp. 83 (1999).

$\mathbb{R}$. Lacey, et al., Dynamics of the radial flow of strange particles,Proc. of the Relativistic heavy ion minisymposium at the centennial mtg. of the American Physical Society, Atlanta, GA., Mar. 1999 (World Scientific, Signapore), pp. 88 (1999).

$D$. Best, et al., Strangeness in Au+Au collisions between I and $10 \mathrm{~A} \mathrm{GeV,} \mathrm{Proc.} \mathrm{of} \mathrm{the} \mathrm{Relativistic} \mathrm{heavy} \mathrm{ion}$ minisymposium at the centennial mtg. of the American Physical Society, Atlanta, GA., Mar. 1999 (World Scientific, Signapore), pp. 286 (1999).

G. Rai, et al., Results from the experiment 895 at the BNL AGS, Proc. of Quark Matter '99, Torino, Italy, May, 1999, Nucl. Phys. A661, 162c (1999).

M. A. Lisa, et al., Beam energy evolution of HBT systematics at the AGS, Proc. of Quark Matter '99, Torino, Italy, May 1999, Nucl. Phys A661, 444c, (1999).

C. Pinkenburg, et al., Elliptic flow: transition from out-of-plane to in-plane emission in Au+Au collisions, Phys. Rev. Lett. 83, 1295 (1999).

M.A. Lisa, et al., The bombarding energy dependence of pi interferometry at the AGS, Phys. Rev. Lett. 84, 2798 (2000).

H. Liu, et al., Sideward flow in Au + Au collisions between $2 A$ GeV and $8 A$ GeV, Phys. Rev. Lett. 84, 5488 (2000).

P. Chung, et al., Anti-flow of $K^{\circ}$ mesons in 6A GeV Au + Au collisions, Phys. Rev. Lett. 85, 940 (2000).

S. Y. Panitkin, et al., Probing baryon freeze-out density at the AGS with proto correleations, Proc. of XXIX Intl. Symposim of Multiparticle Dynamics, Brown U., Provident, Rhode Island. Aug. 1999 (World Scientific, Singapore) pp. 208 (2000)

S. Y. Panitkin, et al., Model-independent source imaging using two-pion correlations in 2 to $8 \mathrm{~A} \mathrm{GeV} \mathrm{Au+Au}$ collisions. (submitted for publication) (2000).

K.A. Lisa, et al., Azimuthal dependene of pion interferometry at the AGS, Phys. Lett. B 496, 1 (2000).

P. Chung, et al., Directed flow of Ahyperons in (2-6)A GeV Au + Au collisions, Phys. Rev. Lett. Vol. 86, 12, pp. 2533, (2001).

G. Rai (for the E895 collaboration), Hadronic flow in 2-8A GeV Au+Au collisions, Nucl. Phys. A., Vol. 681, pp. 181-189, (2001).

896 S. Costa, Alla ricerca della particella HO all' AGS di Brokhaven, Proc. of 81 st. Ttalian Physical Society, Perugia, Italy, Oct. 2-7, 1995. 
S. Paganis, J. Hoffmann, P. Jensen, J. Schambach, J. Tang, R. Jensen, P. Riley, BNL E896-I: Monte Carlo simulations, Abstract for Joint Spring Mtg of Texas Section of American Physical Society, Abilene, Texas, Mar. 15-16, 1996.

W. J. Llope, The BNL-AGS experiment 896, Proc. 12th Winter Workshop Dynamics, Snowbird, Utah, W. G. Westfall, eds (Plenum Press, N.Y., N.Y.) Feb. 3-10, 1996.

E. Judd, The E896 experiment - search for the H-dibaryon, Conf. Proc. At HIPAGS'96, Detroit, Mich., Aug. 2224, 1996, C. Pruneau, et al eds.

$\mathrm{H}$. Caines, et al., First results from the $H_{o}$ di-baryon search and hyperon production measurements by the AGS experiment 896, Nucl. Phys. A, Vol. 661, pp. 170-176, (1999).

G. Lo Curto, et al., Strange and multi-strange baryon measurement in $A u+A u$ collisions at $11.6 \mathrm{~A}(\mathrm{GeV} / \mathrm{c})$ with the silicon drift detector array from the AGS experiment E896, Nucl. Phys. A, Vol. 661, pp. 489-492, (1999).

898 B. M. Sutherland, P. V. Bennet and J. C. Sutherland, Quantitation of double strand breaks induced in human DNA by centigray doses of 56FE ( 1 GeV/NUCLEON), Analytical Biochemistry 239, ; 53-60 (1996).

C. Zeitlin, L. Heilborin and J. Miller, Detailed characterization of the $1087 \mathrm{MeV} /$ nucleon ${ }^{56} \mathrm{Fe}$ beam used for radiobiology at the AGS, Radiat. Res 149, 387 (1998).

M. Löbrich, B. Rydberg and P. K. Cooper, Non random distribution of DNA double-strand breaks induced by particle irradiations, Intl. Journal of Radiation Biology, Vol. 70, No. 5, p 493-503, (1996).

J. A. Joseph, S. Erat and B. M. Rabin, CNS effects of heavy particle irradiation in space: behavioral implications, Adv. Space. Res. (1997).

M. Durante, K. George and T. C. Yang, Biodosimetry of ionizing radiation by selective painting of prematurely condensed chromosomes in human lymphocytes, Rad. Res. 148, S45-S50, (1997).

$\mathrm{H}$. Wu, M. Durante, K. George and T. C. Yang, Induction of chromosome aberrations in human cells by charged particles, Rad. Res. 148, S102-107, (1997).

S. B. Curtis, M. E. Vazquez, J. W. Wilson, W. Atwell, M. Kim, J. Capala, Cosmic ray hit frequencies in critical sites in the central nervous system, Adv. Space Res. Vol. 22 (2), p. 197-207 (1998).

M. E. Vazquez, Basic neurobiological problems in long-term deep space flights, Adv. Space Res. Vol. 22 (2), p.171-183, (1998).

$900 \mathrm{~K}$. Kwiatkowski, et al., Energy dissipation and multifragment decay in the ${ }^{3} \mathrm{He}+{ }^{\text {nat }}$ Ag system, Phys. Rev. C49, 1516 (1994).

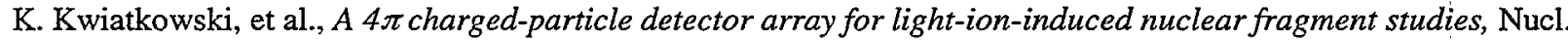
Instr. Meth. A 353, 212 (1994).

K. Kwiatkowski, et al., Multifragmentation in the $4.8 \mathrm{GeV}^{3} \mathrm{He}+{ }^{n a t} \mathrm{Ag},{ }^{197} \mathrm{Au}$ reactions, Phys. Rev. Lett. 74, 3756 (1995).

K. Kwiatkowski, et al., The Indiana silicon sphere $4 \pi$ charged-particle detector array, Nucl. Instr. Meth. A 360 , 571 (1995).

K. B. Morley, et al., Saturaton of deposition energy in relativistic ${ }^{3} \mathrm{He}$-induced reactions, Phys. Lett B 355,52 (1995). 
D. S. Bracken, Charging effects in passivated silicon detectors, Nucl. Inst. Meths. Phys. Res. A 365, 424 (1995). G. Wang, Cavitation and penetration in central collisons wth light ions, Phys. Rev. C. 53, 1811 (1996).

K. B. Morley, et al., $4 \pi$ studies of the $1.8-4.8 \mathrm{GeV}^{3} \mathrm{He}+{ }^{n a t} \mathrm{Ag},{ }^{197} \mathrm{Au}$ reactions. I. Energy deposition, Phys. Rev. C 53, 1811 (1996).

E. Renshaw, et al., $4 \pi$ studies of the $1.8-4.8 \mathrm{GeV}{ }^{3} \mathrm{He}+{ }^{\mathrm{nat}} \mathrm{Ag},{ }^{197} \mathrm{Au}$ reactions. II. Multifragmentation, Phys. Rev. C 54, 749 (1996).

W. Hsi, et al., Hadron-induced multifragmentation, Proc. of XIII Winter Workshop on Nuclear Dynamics, Marathon, FL, Feb. 1-8, 1997.

W. Hsi, et al., Formation of hot nuclei with GeV $\rho$ and $\pi^{*}$ beams. Physical Review Letters, Vol 79, No. 5, p817, Aug 4, 1997.

G. Wang, et al., Time dependence of multifragmentaton in light-ion-induced reactions, Phys. Lett. B393, 270 (1997).

V. E. Viola, et al., Probing the nuclear EOS with GeV light ion beams, Nucl. Phys. A626, 287C (1997).

K. Kwiatkowski, et al., Light-ion-induced multifragmentation, Proc. 35th Intl. Winter Mtg. on Nuclear Physics, Bormio, Italy, Feb. 1997, ed. I. Iori, p. 432.

K. Kwiatkowski, et al., Multifragmentation: Thermal vs. Dynamic effects, Proc. of 6 th Intl. Conf. on NucleusNucleus Collisions, June 2-6, 1997, Gatlinburg, TN.

V. E. Viola, et al., Heating the nuclear liquid with GeV hadrons, Proc. of 8th Intl. Conf. on Nuclear Reaction Mechanisms, Varenna, Italy, June 9-14, 1997.

J. Zhang, et al., Complex fragment emission in the $200-\mathrm{MeV}^{4} \mathrm{He}+{ }^{\text {nat }} \mathrm{Ag},{ }^{197}$ Au reactions, Phys. Rev. C56, 1918 (1997).

$\mathrm{K}$. Kwiatkowski et al., Heating the nuclear liquid with GeV hadrons, Proc. 8th Intl. Conf. Nuclear Reaction Mechanisms, Varenna, Italy, June 9-14, 1997, ed. E. Gadiolo, p.198.

W-C. Hsi, et al., Hadron-induced multifragmentaiton, Advances in Nuclear Dynamics 3 (eds. W. Bauer and A. Mignerey, Plenum Press, New York), p. 197 (1997).

W. C. Hsi, et al., Sideways-peaked angular distributions in hadron-induced multifragmentation: shock waves, toroids or kinematics?, Indiana Report INC-40007-126 (1997), Phys. Rev. C 58, R13 (1998).

V. E. Viola, K. Kwiatkowski, Isotope-ratio thermometers: are they a valid gauge of nuclear temperature? Indiana Nuclear Chemistry Report INC-40007-116 (1998).

D. S. Bracken, et al., Moving source analysis of exclusive events in $\mathrm{GeV}^{3} \mathrm{He}$-induced reactions, Indiana Nuclear Chemistry Report INC-40007-121 (1998).

V. E. Viola, et al., Heating nuclei with $8 \mathrm{GeV} / \mathrm{c} \pi$ and $\bar{p}$ beams, Proc. of 5 th Biennial Conf. of Low-Energy Antiproton Physics, Cagliari, Italy, Sept. 1998.

K. Kwiatkowski, et al., Dynamic and statistical effects in hadron-induced multifragmentation, Proc. 14th Winter Workshop on Nuclear Dynamics, Snowbird. Utah, (1998).

K. Kwiatkowski, et. al., Dynamic and statistical effects in light-ion-induced multifragmentation, Advances in Nuclear Dynamics 4, Plenum Press, New York, p173-181 (1998). 
V.E. Viola and K. Kwiatkowski, How to boil a nucleus, AmericanScientist 86, p449-455 (1998).

W-C. Hsi, et. al., Sideways-peaked angular distributions in hadron-induced multifragmentation: shock waves, geometry, or kinematics?, Physical Review C58, pR13-R17 (1998).

T. Lefort, et al., Heating of nuclei with $8 \mathrm{GeV} / \mathrm{c}$ antiprotons, Proc. of $5^{\text {th }}$ Biennial Conf. On Low Energy Antiproton Physics, Cagliari, Italy, Sept 7-12, 1998.

L. Beaulieu, et. al., Heating of nuclear matter and multifragmentation: antiprotons $v$ s. pions, proceedings of $15^{\text {th }}$ Winter Workshop on Nuclear Dynamics, Park City, UT., January 9-16, 1999.

V. E. Viola, et. al., Isolating the thermal degree of freedom in nuclear multifragmentation, Proc. of Multifragmentation Intl. Workshop 27 on Gross Properties of Nuclei and Nuclear Excitations, Hirschegg, Kleinwalsertal, Austria, January 17-23, 1999.

T. Lefort, Heating ${ }^{197}$ Au nuclei with $8 \mathrm{GeV}$ antiprotons and $\pi^{-}$beams, proceedings of $37^{\text {th }}$ Winter Mtg on Nuclear Physics, Bormio, Italy, Jan. 25-30, 1999.

T. Lefort, Heating ${ }^{197}$ Au nuclei with $8 \mathrm{GeV}$ antiprotons and $\pi^{-}$beams, Proc. $37^{\text {th }}$ Intl. Winter Mtg. on Nuclear Physics, Bormio, Italy, Jan. 25-30, 1999.

W.-C. Hsi, et al., Exclusive studies of angular distributions in GeV hadron-induced reactions with ${ }^{197}$ Au, Phys. Rev. C60, 034609 (1999).

R. Yanez, Experimental evidence for dynamical decay of nuclear matter, Phys. Rev. Lett 82, 3585 (1999).

G. Wang, et al., Source size and time dependence of multifragmentation induced by $\mathrm{GeV}^{3} \mathrm{He}$ beams, Phys. Rev. C60, 014603 (1999).

V. E. Viola, K. Kwiatkowski and W. A. Friedman, Double isotope-ratio thermometers: the influence of emissiontime scales, Phys. Rev. C59, 2660 (1999).

M. Colonna, et al., Measurements of low-energy $(d, n)$ reactions for BNCT, Med. Phys. 26, 793 (1999).

Y. Larochelle, et al., Probing mid-rapidity source characteristics with charged particles and neutrons in the

${ }^{35} \mathrm{CI}+$ Ta reaction at $43 \mathrm{MeV} /$ nucleon, Phys. Rev. C59, $\mathrm{R} 565$ (1999).

C. Williams, et al., Reply to comment on fragment distributions for highly charged systems, Phys. Rev. C59,552 (1999).

$\mathrm{X}$. Qian, et al., The production and decay of excited quasi-projectiles in peripheral and semi-peripheral ${ }^{35} \mathrm{CI}+{ }^{197} \mathrm{Au}$ reactions in Fermi energy domain, Phys. Rev. C59, 269 (1999).

L. Beaulieu, et al., Heating of nuclear matter and multifragmentation: antiprotons $v$ s pions, Proc. of $15^{\text {th }}$ Winter Workshop on Nuclear Dynamics, Park City, Utah, Jan. 9-16, 1999, eds. W. Baeur and G. D. Westfall, Kluwer Publication (1999)

L.G. Moretto, et al., Statistical exploration of fragmentaiton phase space in multifragmenting sources, Proc. of Intl. Workshop 27 on Gross Properties of Nuclei and Nuclear Excitations, Hirschegg, Austria, Jan. 17-23, 1999, eds. H.Feldmeier, J. Knoll, W. Norenberg and J. Wambach, GSI, Darmstadt, p. 192 (1999).

T. Lefort,et al., Thermally-induced expansion in the $8 \mathrm{geV} / \mathrm{c}+{ }^{197}$ Au reaction, INC-40007-141, Phys. Rev. C RC (in press).

T. Lefort, et al., Heating ${ }^{197} \mathrm{Au}$ Nuclei with GeV/c antiproton and beams, Phys. Rev. Lett. 83, 4033-4036 (1999). 
L.Beaulieu, et al., Thermal excitation of heavy nuclei with 5-15 GeVc antiproton, proton and pion beams, Phys. Lett. B. 163159 (1999).

W.C. His, et al., Exclusive studies of angular distributions in GeV hadron-induced reactions with ${ }^{197} A u$, Phys. Rev. C 60, 034609-1 034609-10 (1999).

K.Kwiatkowski, et al., Multifragmentation with GeV light-ion beams, Nucl. Phys., A654, 786c-791c (1999).

L.Beaulieu, Transition from surface to bulk emission in thermal multifragmentation, INC-40007-140, Phys. Rev. Lett. 84, 5971 (2000).

T. Lefort, Thermally-induced multifragmentation in the $8 \mathrm{GeV} / \mathrm{c}+{ }^{-197} \mathrm{Au}$ reacton, Proc. $38^{\text {th }}$ Intl. Winter Mtg. on Nuclear Physics, Bormio, Ialy, January 2000.

V.E. Viola, Signals for the transition from liquid to gas in hot nuclei, E900:5-15 GeV/c $\overrightarrow{\mathrm{p}}+{ }^{197} \mathrm{Au}$, CRIS 20000 Phase Transitions in Strong Interactions: Status and Perspectives, Catania, Italy May 2000 (Elsevier, to be published).

V.E.Viola, Phase transition signals in thermally excited nuclei, E900: 5-15 GeV/c $\overline{\mathrm{p}}$ and ${ }^{197} \mathrm{Au}$, Bologna $2000-$ Structure of the Nucleus at the Dawn of the Century, Bologna, Italy, May 29-June 3, 2000 (to be published).

L. Beaulieu, Transition from surface to bulk emission in thermal multifragmentation, INC-40007-140, Phys. Rev. Lett. 84, p5971 (2000).

903 P. D. Panetta, J. E. Ostenson, D. K. Finnemore and C. L. Snead, Jr., Pinning mechanisms in $\mathrm{Y}_{1} \mathrm{Ba}_{2} \mathrm{Cu}_{3} \mathrm{O}_{7}$ single crystals, Phys Rev. B1 52, 15, 570, (1995).

904 R. Weinstein, Very high trapped fields: cracking, creep, and pinning centers, invited paper, Proc. of 10th Anniversary HTS Workshop on Physics, Materials and Applications, Houston; TX, edited by W. K. Chu, D.Gubsner and K. A. Miller, World Scientific Press, p. 625, (1996).

R. Weinstein, The pole of uranium with and without radiation, in the achievement of $J_{c}{ }^{\prime} 1-^{5} A c u$ in large grain,HTS, invited paper, Proc. Of 1997 Workshop on Processing Superconducting (RE) BCO Large Grain materials, Cambridge, UK, July, 1997.

905 T. Nagae, Few-body hypernuclear systems, Nuclear Physics A631, 363c-375c (1998).

T. Nagae, et al., Observation of $a_{\Sigma}{ }^{4} \mathrm{He}$ bound state in the ${ }^{4} \mathrm{He}\left(\mathrm{K}^{*}, \pi^{-}\right)$reaction at $600 \mathrm{MeV} / \mathrm{c}$, Phys. Rev. Lett, 80 , p. $1605,(1998)$.

906 Y. Yamamoto, M. Wakai, T. Motoba, T Fukuda, Prodution of double-lambda hypernuclei at $\left(\mathrm{K}^{*}, \mathrm{~K}^{+}\right)$reaction points and their pionic decays, published in Nucl. Phys. A625, 107-142, (1997).

907 M.W. Ahmed (for the E907 collaboration), Characteristics of an active chamber target to locate the reaction vertex in the $\left(K, \pi^{\circ}\right)$ reaction, proc. of the conf. on Kaon and Hypernuclear Physics, BNL, (1997), Nucl. Phys. A639, p. 117-120 (1998).

A. Rusek (for the E907 collaboration), ( $\mathrm{K}_{\text {STOP }}^{-}, \pi^{\circ}$ ) with the neutral meson spectrometer, Proc. of the conf. on Kaon and Hypernuclear Physics, BNL, (1997), Nucl. Phys. A639, p. 111-116 (1998).

910 I. Chemakin, et al., Strange particle production and an H-dibaryon search in p-A collisions at the AGS, Nucl. Phys. A, vol. 639, pp. 407-416, (1998).

B. A. Cole (for the E910 collaboration), Studying heavy ion physics at the AGS using proton-nucleus collisions, Nucl. Phys. A., Vol. 638, pp. 423-426, (1998). 
I.Chemakin, et al., Measuring centrality with slow protons in proton-nucleus collisions at $18 \mathrm{GeV/c}$, Phys. Rev. C, Vol. 60, 024902, (1999).

B.A. Cole (for E910 collaboration), Constituent quarks and proton break-up in p-A collisions at the AGS, Nucl. Phys. A., Vol. 661, pp. 366-369, (1999).

I.Chemakin, Semi-inclusive $\Lambda$ and $K_{S}$ production in $p-A u$ collisions at $17.5 \mathrm{GeV} / \mathrm{c}$, Phys. Rev. Lett., Vol. 85, 23 , pp. 4868, (2000).

913 B. M. K. Nefkens, $N^{*}$ physics at the AGS with the crystal ball multiphoton spectrometer, Inst. for Nucl. Theory, Vol IV, T-S.H. Lee, W. Roberts eds., World Scientific, pg. 186, (1996).

V. Abaev and B. M. K. Nefkens, $S$-wave resonance coupled-channel approach to the reactions $\pi+p \rightarrow \eta+n$ and $K+p \rightarrow \eta+\Lambda$, and a determination of the eta- $n$ and eta lambda scattering lengths, Phy. Rev. C53, 385 (1996).

W. J. Briscoe, Meson production experiments at TJNAL and BNL, Inst. for Nucl. Theory, Vol. IV, T-S. H. Lee, W. Roberts, eds., World Scientific, pg. 306, (1996).

L.X. Jian, L. Addessi, V. Castillo, L.H.Gong, J. Leskowicz, R. Meier, G. Miglionico, J. Scaduto, Safety design, operation, and control of a liquid hydrogen target at BNL. Presented at 1997 Cryogenic Engineering Conf., Portland, Oregon, July 28-August 1, 1997.

W. B. Tippens, (for 913 collaoboration), Hadron spectroscopy with the crystal ball at the AGS, Presented at Hadron 97, S.U. Chung, ed. (1997).

A. Starostin, Eta production in the reastion $\pi^{-} p$ to $\eta N$ near threshold, presented at Hadron 97, S.U. Chung, ed. (1997).

W. B Tippens, (for the 913 collaboration) Recent results from the crystal ball program at BNL, proc. of GW/TJNAF Workshop on $\mathrm{N}^{*}$ Physics, Oct. 30 - Nov. 1, 1997.

S. Prakhov, et al., Search for the CP forbidden decay $\eta \rightarrow 4 \pi^{0}$. Phys. Rev. Lett., Vol. 84, 21, pp. 4802, (2000).

A. Starostin, et al., Measurmeent of $\pi^{0} \pi^{0}$ production in the nuclear medium by $\pi^{-}$interactions at $0.408 \mathrm{GeV} / \mathrm{c}$, Phys. Rev. Lett., Vol. 85, 26, pp. 5539, (2000).

916 Y. D. He, P. B. Price, and M. Solarz, Charge-changing cross sections for gold projectile at $-4 \mathrm{~A}$ GeV in various targets, (1997).

917 C. Ogilvie (for the E917 collaboration), E917 collaboration: probing the dynamics of HI collisions plus searching for the QGP, Proc. Of HIPAGS 96, WSU-NP-96-16, C. A. Pruneau, ed., Detroit, Mich., (1996).

J. Chang (for the E917 collaboration), Multiplicity measurements at various AGS beam energies, Proc. 13th Winter Workshop on Nuclear dynamics, Marathon, Fla., (1997).

J. Dunlop (for the E917 collaboration) An excitation function of particle production at the AGS, Proc. of 6th conf. On Intersections of Particle and Nuclear Physics, Big Sky, MT., (1997).

B. Back, et al., A beam vertex detector using scintillating fibers, accepted for publication in Nuclear Instru. and Meth A, (1997).

C. Ogilvie (for the E917 collaboration), E802/859/866/917 report, Proc. of 13th Intl. Conf. on Ultra-Relativistic Nucleus-Nucleus Collisions, Tsukuba, Japan, (1997). 
R. Set and J. Dunlop (for the E917 collaboration), An excitation function at the AGS: Probing the dynamics of heavy ion collisions, Proc. of 13th Intl. Conf. on Ultra-Relativistic Nucleus-Nucleus collisions, Tsukuba, Japan, (1997).

923 M. V. Diwan, et al., Search for T-violation in KMU3 decay, presented by Hong Ma, Div. Of Particles and Fields, APS 1996 Divisional Mtg., Minneapolis, MN., Aug. 10-15, 1996.

R. Adair, et al., Muon polarization working group report, AGS2000 Workshop, Workshop on AGS Experiment for the 21st Century, May 13-17, 1996, BNL.

M. Diwan, Search for T-violation in Kmu3 decay, Intl. Workshop on Kaon Muon and Neutrino Physics and the Future, KEK, October 31 - November 1, 1997.

924 D. W. Hertzog, P. T. Debevec, R. A. Eisenstein, M. A. Graham, S. A. Hughes, P. E. Reimer, and R. L. Tayloe, A high resolution lead/scintillating fiber electromagnetic calorimeter, University of Illinois at Urbana-Champaign, Nuclear Physics Laboratory Department of Physics P/90/3/41.

925 P. R. Cameron, M. M. Blaskiewicz, et al, Polarized beam as the pump in a parametric amplifier, RHIC/AP/124, Oct. 1997.

K. Bai, et al., Overcoming intrinsic spin resonances with an rf dipole, Physical Review Lett., Vol 80, No. 21, 4673-4676, May 25, 1998.

Y. Makdisi, et al., Asymmetry in inclusive $\pi^{+}$, p production at $22 \mathrm{GeV}$ BNL E925, Proc. of SPIN $98,13^{\text {th }}$ Intl. Symposum on High Energy SPIN Physics, Sept. 8-12, 1998, Protvino, Russia, pp. 474-476.

$\mathrm{K} . \mathrm{Krueger}$, et al., Large analyzing power in inclusive $\pi^{+}$production at high $X_{F}$ with a $22 \mathrm{GeV} / \mathrm{c}$ polarized proton beam, Phys. Lett. B459, p. 412 (1999).

H. Spinka, et al., $A_{N}$ for inclusive $\pi^{+}$production at $21.6 \mathrm{GeV} / \mathrm{c}$ from $C$ and $L H 2$, Proc. SPIN $2000,14^{\text {th }}$ Intl. SPIN Physics Symposium, Oct. 16-21, 2000, Osaka, Japan.

926 A. Konaka, $\mathrm{K}_{\mathrm{L}}^{\mathrm{O}} \rightarrow \pi^{0} v \bar{v}$ at the AGS, presented at the Intl. Workshop on CP Violation in K, Dec. 18-19, 1998, KEK, Tanashi, Tokyo, Japan (1998).

929 S. Ajimura, et al., Observation of Spin-Orbit Splitting in A Single-Particle States, Phys. Rev. Letters, Vol. 86, 19, May 7, 2001.

930 H. Tamura, et al., High-resolution hypernuclear gamma-ray spectroscopy, Nuclear Physics, A663, pp 481c-484cm (2000).

K. Tanida, et al., (for KEK E419 and BNL E930 collaborations), Hypernuclear gamma-ray spectroscopy experiments with germanium detectors", Proc. APCTP Workshop on Strangeness Nuclear Physics (SNP'99), Seoul, Korea, Feb. 19-22, 1999, Eds. I.T. Cheon, S.W. Hong and T. Motoba, p.98 (2000).

H. Tamura, et al (for KEK E419 and BNL E930 collaborations) High resolution gamma-ray spectroscopy of ${ }^{7} \mathrm{Li}$ and ${ }^{9} \mathrm{Be}$, Few-Body Systems Suppl. 12 p. 342 (2000).

K.Tanida, et al (for KEK E419 and BNL E930 collaborations), Lambda-N spin dependent interactions studied by gamma-ray spectroscopy of hypernclei, proc. RCNP-TMU Symposium on Spins in Nuclear and Hadronic Reactions, Oct. 26-28, 1999, Tokyo Metro. U. (2000).

K. Tanida et al (for KEK E419 and BNL E930 collaborations) "High-resolution gamma-ray spectroscopy of hypernuclei with germanium detectors", proc. $16^{\text {th }}$ Intl. Conf. on Few Body Problems, March 2000, Taipei; Nucl. Phys. A684, 560c (2001). 
H. Tamura, et al., (for KEK E419 and BNL E930 collaborations) "Hypernuclear gamma spectroscopy - Recent results with HYPERBALI" Proc. Intl. Conf. on Structure of the Nucleus at the Dawn of the Century, May-June, 2000 , Bologna, Italy (in press).

H. Tamura, Hypernuclear physics with hadronic beams", proc. Workshop on Hypernuclear Physics with Hadronic probes, Hampton, England, December 1999 (in press).

H.Tanida, et al., High-rsolution gamma-ray spectroscopy of 7-Lamda-Li, Proc. Int. Conf. of Hypernuclear and Strange Particle Physics, (HYP2000), Torino, Oct. 2000, Nucl. Phyas. A691, 115c-118c (2001).

H. Akikawa, et al (for E930 collaboration), "Gamma ray spectroscopy of Lambda Be", proc. $7^{\text {th }}$ Instl. Conf. on Hypernuclear and Strange Particle Physics, Torino, Oct. 23-27, 2000, Nucl Phys. A. 691, 134c-137c (2001).

H.Tamura, "High resolution spectroscopy of Lambda hypernuclei: present status and perspectives", Proc. $7^{\text {th }}$ Intl. Conf. of Hypernuclear and Strange Particle Physics, Torino, Oct. 23-27, 2000, Nucl Phys. A. 691, 76c-84c (2001).

H. Tamura, Impurity Nuclear Physics-Hypernuclear gamma spectroscopy and future plans for neutron-rich hypernucleai, Proc. Intl. Symp. On Perspectives in Physics with Radioactive Isotope Beams (RIB00), Hayama, Nov. 2000.

H. Nakashima, et al., Measurement of incident proton beam characteristics for AGS spallation target experiment, Proc. of 14th Intl. Collaboration on Advanced neutron sources, June 14-19, 1998, Starved Rock Lodge, Utica, Illinois.

$\mathrm{H}$. Takada, et al., Measurement of reaction rate distributions on a mercury target bombarded wit high energy protons, Proc. of 14th Intl. Collboraiton on Advanced Neutron sources, June 14-19, 1998, Utica, Illinois!

M. Futakawa, K. Kikuchi, H. Conrad (for 938 collaboration) Measurement of pressure wave in mercury target, Proc. of 14th Intl. Collaboration on Advanced neutron sources, June 14-19, 1998, Starved Rock Lodge, Utica, Illinois.

R. D. Neef (for 938 collaboration) Spallation neutron target experiments at the AGS-BNL, Niuclear Physics Spring Meeting, Bochum, Germany, March 16-20, 1998, ISSN 0420-0195.

R. D. Neef, Radiation physikcs experiments to develop the target-moderator-reflector system for ESS, Proc. of 14th Intl. Collaboration on Advanced neutron sources, June 14-19, 1998, Starved Rock Lodge, Utica, Illinois.

D. Filges, R. D. Neef and H. Schaal, Radiation physics and nuclear assessment of the target station of the. European spallation environments, SARE-4, Sept. 14-15, 1998, Knoxville, TN.

D. Filges, R. D. Neef and G. Sterzenbach, Experimental validation of nuclear models for the optimiaation of the ESS-target system, Second Intl. Topical Conf. on Nuclear Applications of Accelerator Technology, Sept. 20-23, 1998, Gatlinburg, TN.

E. A. Jerde and D. C. Glasgow, Neutron activation by neutrons produced via proton-induced spallation in a liquid-mercury target: measurements and uncertainties, Journal of Radioanalytical and Nuclear Chemistry.

940 W. Molzen, Improved tests of muon and electron number conservation in muon processes, Proc. of 1997 SLAC Summer School Topical Conf. 1998.

W. Molzon, Physics with low energy muons at the front end of the muon collider, Proc. Workshop on Physics at the First Muon Collider and the Front End of the Muon Collider (1998) 
M.Bachman, The MECO muon beam, Proc. Workshop on Physics at the First Muon Collider and at the Front End of the Muon Collider (1998).

T. J. Liu, MECO physics background studies, Proc. Workshop on Physics at the First Muon Collider and at the Front End of the Muon Collider (1998).

R. Djilkibaev, MECO muon yield simulation using experimental data, Proc. Workshop on Physics at the First Muon Collider and at the Front End of the Muon Collider (1998).

J. Sculli, $\square \square$ conversion status and prospects, Proc. Workshop on Physics at the First Muon Collider and at the Front End of the Muon Collider (1998).

W. Molzon, Improved tests of muon and electron number conservation in muon processes, Proc. of Recontre de Moriond 1998: Electroweak Interactions and Unified Theories, (1998)

W. Molzon, Search for muon and electron lepton number violation with MECO at BNL, Proc. Intl. Symposium on Lepton and Baryon Number Violation, Trento, Italy (1998)

949 L. Littenberg, BNL future plans, Proc. Intl. KEK Workshop on kaon, Muon, Neutrino Physics and Future, KEK Proc. 97-124, JHF-97-8, 27, eds. Y. Kuno and T. Shinkawa (1998).

S. Kettell, Rare and forbidden kaon decays at the AGS, Proc. Summer Inst. on Part. Physics, eds. A. Breaux, J. Chan, L. DePorcel and L. Dixon, SLAC-R-528, CONF-9708161, UC-414, 305, BNL-65021 (1998).

T. K. Komatsubara (for the collaboration), Status of the study of the rare decay $K+\rightarrow P I+$ neutrino anti-neutrino at BNL, presented at $17^{\text {th }}$ Intl. Workshop on Weak Interactions and Neutrinos (WIN 99) Cape Town, S. Africa, Jan. 24-30, 1999, e-Print Archive: hep-ex/9905014 (1999).

S. Kettell, Rare kaon decays, Proc. of $3^{\text {rd }}$ Intl. Conf. and CP Ciolation, Taipei, Taiwan, eds. H.Y. Cheng and W.S. Hou (2000).

L. Littenberg, Rare kaon decays, Proc. $35^{\text {th }}$ Rencontres de Moriond, Les Arcs, France, ed. J. Tran Thanh Van (2000).

S. Kettell, Future kaon programs at BNL and FNAL, $7^{\text {th }}$ Intl. Conf. on the Intersections of Particle and Nuclear Physics, Quebec City, Canada, eds. W. J. marciano and Z. Parsa.

MPS S. Eiseman, et al., The MPS II drift chamber system. Nuclear Instruments and Methods 217, 140-148 (1983). 


\section{$C-A D$ Experimenters}

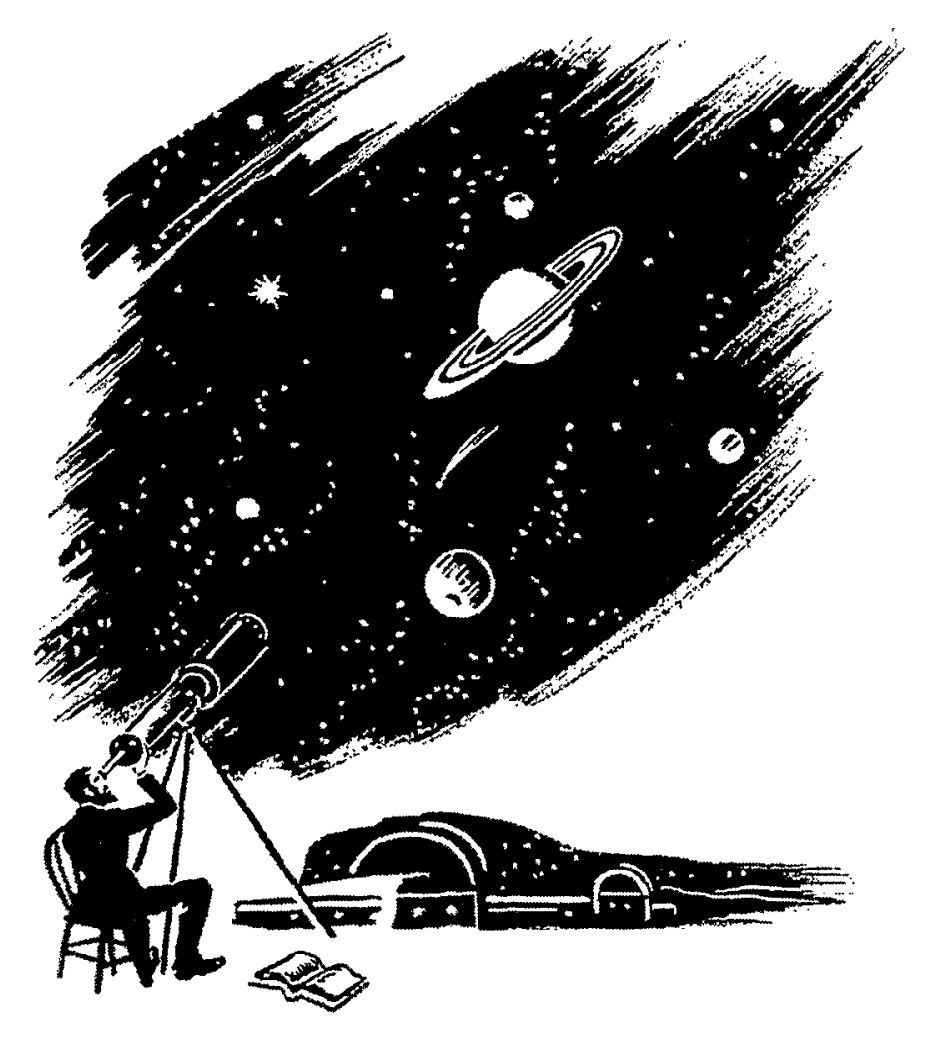

Name

Affiliation

Experiment Number 

Abaev, V. V.

Ables, E.

Aclander, $\mathrm{H}$.

Adair, R.

Adams, G.

Adams, J. H.

Adams, $N$.

Adams, $\mathrm{T}$.

Adler, C.

Adler, S.

Adomava, D.

Agakishiev, $\mathrm{H}$.

Ahammed, Z.

Ahle, L.

Ahmad, S.

Ahmed, M.

Ahn, J. K.

Ahrens, L.

Aiello-Lammens, $M$.

Ajimura, $S$.

Ajitanand, $N$.

Akchurin, N.

Akekseev, I. G.

Akiba, Y.

Akiba, Yasuyuki

Akikawa, $\mathrm{H}$.

Albergo, S.

Alburger, D.

Alessi, J.

Alexander, J.

Allen, $M$.

Allgower, C.

Allgower, $\mathrm{C}$.

Alrick, $\mathrm{K}$.

Alster, J.

Alyea, J.

Alyushin, M.

Amaglobeli, N.

Amann, J.

Ambrose, D.

Amelin, N.

Amonett, J.

Ananeva, M.A.

Anderson, B. D.

Anderson, $\mathrm{M}$.

Andreev, $Y$.

Andrianov, Fedor

Androic, $D$.

Ankenbrandt, C.

Antonelli, $F$.
Petersburg Nuclear Physics Institute

$954,953,924,914,913,909,890$

Lawrence Livermore National Laboratory

933

850

Yale University

923

Rensselaer Polytechnic Institute

852

Naval Research Laboratories

934

Rice University

STAR

University of Notre Dame

852

University of Frankfurt

STAR

787

Laboratory of High Energy Physics - Dubna

STAR

STAR

Laboratory of High Energy Physics - Dubna

STAR

866

Massachusetts Institute of Technology

891

Rice University

University of Houston

Kyoto University

$931,929,907$

906

Brookhaven National Laboratory

925,880

PHENIX

Osaka University

State University of New York-Stony Brook

$930,929,906$

895

pp2pp, 925

925

917,866

PHENIX

906

896

885

Brookhaven National Laboratory

Brookhaven National Laboratory

$951,925,880$

895

STAR

880

STAR

939

850

927

STAR

865

HEP Inst. Tibilsi State University

Los Alamos National Laboratory

$933,931,907$

871

University of Texas at Austin

STAR

STAR

STAR

Kent State University

Particle Physics Laboratory - Dubna

Kent State University

University of California - Davis

STAR, 948

STAR

923

Inst. Nuclear Research, Moscow

PHENIX

Petersburg Nuclear Physics Institute, Russia

931,907

Fermi National Laboratory

932

957 
Aoki, M.

Aphecetche, Laurent

Appel, R.

Arai, Y.

Archambeau, J.

Ardebili, M.

Argintaru, D.

Armendariz, J.

Armstrong, $\mathrm{T}$.

Arnold, L.

Asano, $\mathrm{Y}$.

Ashery, D.

Ashktorab, K.

Aslanyan, P.

Assamagan, $\mathrm{K}$.

Asselta, K.

Athana, M. J.

Atiya, M. S.

Atoyan, G. S.

Aufderheide, M. B.

Averichev, G.

Azmoun, Babak
KEK, National Laboratory for High Energy Physics

Subatech

University of Pittsburgh

University of Tsukuba

Loma Linda University

Princeton University

University of Bucharest, Romania

University of New Mexico

Penn State University

Institute Subatomics de Strasbourg

University of Tsukuba

Tel-Aviv University

Brookhaven National Laboratory

Laboratory of High Energy Physics - Dubna

Hampton University

Brookhaven National Laboratory

Carnegie-Mellon University

Brookhaven National Laboratory

Inst. for Nuclear Reserach, Moscow

Lawrence Livermore National Laboratory

Laboratory of High Energy Physics - Dubna

State University of New York at Stony Brook
936,787

PHENIX

865

936

856

787

BRAHMS

865

864

STAR

936

835

866

STAR

865

STAR

788

787

$926,923,865$

933

STAR

PHENIX

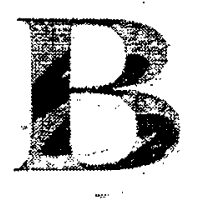

Babintsev, Vladimir

Bachman, M.

Back, B.

Bacow, J.

Bagaturia, Y.

Bagga, R.

Bai, $M$.

Baker, $\mathrm{K}$.

Baker, $\mathrm{M}$.

Baker, $\mathrm{M}$.

Balcer-Kubiczek, E.

Baldwin, A.

Balewski, J.

Barakat, $\mathrm{M}$.

Barannikova, $\mathrm{O}$.

Barber, R.

Barbier, L. M.

Barcellos-Hoff, M. H.

Barish, $\mathrm{K}$.

Barkov, L. M.

Barlett, $M$.

Barnby, L.

Barnes, $P$.

Barnes, $P$.

Barnes Jr., P. D.

Barrette, $\mathrm{J}$.
Inst. for High Energy Pysics, Russia

University of California-Irvine

Argonne National Laboratory

Massachusetts Inst. of Technology

HEP Inst. Tibilsi State University

Abilene Christian University

Argonne National Laboratory/Indiana University

Hampton University

Brookhaven National Laboratory

Massachusetts Institute of Technology

University of Maryland Baltimore County

Kent State University

Indiana Univesity

Louisiana Tech University

Purdue University

University of Houston

Goddard Space Flight Center

Lawrence Berkeley Laboratory

University of California-Riverside

Budker Inst. For Nuclearn Physics

University of Texas

Kent State University

LAMPF

Los Alamos National Laboratory

Lawrence Livermore National Laboratory

McGill University
PHENIX

$940,935,871$

PHOBOS, 917, 900

PHOBOS

865

914,913

$950,925,880$

865

PHOBOS

866

919,898

STAR

STAR, 948

931, 907

STAR

929

934

$957,947,898$

PHENIX, 941, 925, 864

821

829

STAR

811

885,813

933

877 
Bart, S.

Barton, D. S.

Bar-Yam, Z.

Bassalleck, B.

Bassano, D.

Bathe, Stefan

Batinic, $M$.

Batourine, V.

Batsouli, S.

Batsouli, Sotiria

Baturin, V.

Baublis, Victor

Baudot, J.

Bauer, G.

Bazanko, A.

Bearden, I. G.

Beatty, J. J.

Beavis, D.

Bedford, J.

Bekele, S.

Bekrenev, V. S.

Belaga, V.

Belikov, N. I.

Belkacem, A.

Belli, M.

Bellwied, R.

Belyakov, Igor

Belzer, L. I.

Benante, J.

Bennhold, C.

Bennett, B.

Bennett, G.

Bennett, $M$.

Bennett, $\mathrm{S}$.

Berdoz, A.

Bergbusch, $P$.

Berger, J.

Bergman, D.

Bersch, $\mathbb{R}$.

Bertaina, $M$.

Besliu, C.

Best, D.

Betev, L.

Betts, R.

Betts, R.

Bezverkhny, Betty

Bharadwaj, V.

Bhuyan, B.

Bialas, A.

Bichsel, Hans

Bick, Michael

Bickley, A.

Bidoli, V.

Bieser, F.

Biglan, A.
Brookhaven National Laboratory

Brookhaven National Laboratory

909,856

PHOBOS, 850

University of Massachusetts-Dartmouth

University of New Mexico

SUNY Health Science Center

852

Insitut Fuer Kernphysik

Ruder Boskovic Institute

Pennsylvania State University

$950,949,926,906,885,865,813,811$

PHENIX

Yale University

Columbia University

Pennsylvania State University

Petersburg Nuclear Physics Institute, Russia

Institute Subatomics de Strasbourg

Paul Scherrer Institute

Princeton University

Niels Bohr Inst. for Astronomy, Physics and Geophysics-U.Copenhagen

Pennsylvania State University

Brookhaven National Laboratory

Colorado University

Ohio State University

Petersburg Nuclear Physics Institute

Laboratory of High Energy Physics - Dubna

Institute for High Energy Physics-Protvino

Lawrence Berkeley Laboratory

National Inst. of Health, Rome, Italy

Wayne State University

Inst. for High Energy Pysics, Russia

Moscow State University

$927,914,913,890$

STAR

941

PHENIX

850

PHENIX

STAR

938

787

BRHAMS

934

BRAHMS, 866

957, 947

STAR

$954,953,924,914,913,909,890$

STAR

925

892

957

STAR, $896,877,864$

PHENIX

852

821

George Washington University

Wayne State University

Brookhaven National Laboratory

856,821

896

941

Wayne State University

885,813

Carnegie-Mellon University

949,787

University of Frankfurt

STAR

866

877

941

Massachusetts Int. of Tech.

University of Bucharest-Romania

BRAHMS

Lawrence Berkeley National Laboratory $\quad 895$

University of California-Los Angeles

Brookhaven National Laboratory

925

University of Illinois-Chicago

PHOBOS

Yale University

917

Fermilab

STAR

Brookhaven National Laboratory

880

949

Jagellonian U., Poland

PHOBOS

STAR

University of Washington

PHENIX

University of Maryland

PHOBOS

957

University of Rome, Thor Vergara

STAR

Carnegie-Mellon University 
Billmeier, Anja

Bindel, R.

Binns, W. R.

Bionta, R. M.

Birchall, J.

Bishop, J. M.

Blackmore, E.

Bland, $L$.

Bland, L. C.

Blaszczak, Z.

Blecher, $\mathrm{M}$.

Blyakhman, Y.

Blyth, C.

Bodyagain, V.A.

Boemi, D.

Bogdanov, A. A.

Boggild, $H$.

Bogucki, W.

Bondorf, J. P.

Bonner, B. E.

Bonnet, D.

Borak, $T$.

Boucham,A.

Boudrie, D.

Boudrie, R. L.

Bouillo, N.

Bourotte, J.

Bouvier, S.

Brabson, B. B.

Braby, J.

.Bracken, D. S.

Brady, P.

Braun-Munzinger, $P$.

Bravar, S.

Breuer, $\mathrm{H}$.

Brennan, $M$.

Brill, A. B.

Briscoe, W.

Britt, H. C.

Britt, $\mathrm{H}$.

Brooks, A.

Brown, A.

Brown, C.

Brown, D. H.

Brown, D.S.

Brown, David

Brown, H. N.

Brown, $\mathrm{K}$.

Brown, $R$.

Briscoe, W.

Bruner, N.

Bryman, D.

Bryman, D.A.

Buchnan, J. A.

Budick, B.
Wayne State University

STAR

University of Maryland

PHOBOS

Washington University in St. Louis

934,869

Lawrence Livermore National Laboratory

933

University of Manitoba

906,885

852

University of Notre Dame

$949,926,787$

Brookhaven National Laboratory

STAR

948

Indiana University

Inst. Nuclear Physics, Poland

PHOBOS

936, 926

BRAHMS

New York University

STAR

852

Moscow State University

896

University of Catania

STAR, pp2pp

Moscow Engineering Physics Inst.-Russia

Niels Bohr Inst. for Astronomy, Physics and Geophysics-U.Copenhagen

Inst. Nuclear Physics, Poland

BRAHMS

PHOBOS

Niels Bohr Inst. for Astronomy, Physics and Geophysics-U.Copenhagen

BRAHMS

Rice University

Institute Subatomics de Strasbourg

Colorado State University

SUBATECH-Nantes

STAR, 896, 891, 818

Los Alamos National Laboratory

Los Alamos National Laboratory

STAR

919

STAR

931, 907

SUBATECH-Nantes

Ecole Polytechnique-France

SUBATECH-Nantes

Indiana University

Texas A\&M University

Los Alamos National Laboratory

University of California-Davis

SUNY at Stony Brook

933

STAR

pp2pp

STAR

852

957

900

STAR, 895

Brookhaven National Laboratory

877

STAR

900

932

856

University of Massachusetts

George Washington University

Lawrence Livermore Lboratory

University of Maryland

Washington State University

Los Alamos National Laboratory

Carnegie-Mellon University

Boston University

Northwestern University

New Mexico State University

Brookhaven National Laboratory

Brookhaven National Laboratory

Brookhaven National Laboratory

$954,953,931,927,924,914,913,909,907,905$

866

917

947,919

PHENIX

896

821

852

PHENIX

952,821

925,880

STAR

890

George Washington University

University New Mexico

Rice University

New York University
PHENIX, 926
926,787
949
891

PHENIX, 926
926,787
949
891

PHENIX, 926
926,787
949
891

PHENIX, 926
926,787
949
891

BRAHMS, 866 
Budilov, V.

Particle Physics Laboratory - Dubna

STAR

Budny, $\mathrm{T}$.

Wayne State University

877

Budzanowski, A. Inst. Nuclear Physics, Poland

Buenerd, $M$.

Institute of Sciens Nucleaires-France

PHOBOS

Bullis, J.

Brookhaven National Laboratory

pp2pp

919

Bumazhnov, Vladimir

Bump, Leslie

Inst. for High Energy Pysics, Russia

PHENIX

Yale University

STAR

Bunce, $\mathrm{G}$.

Brookhaven National Laboratory

Burger, $M$.

Freiburg University

0,821

Bürger, $\mathrm{T}$.

Freiburg University

813

Burke, Sean

State University of New York at Stony Brook

885,813

Burns, F.

New York University Medical Center

PHENLX

Busza, W.

Massachusetts Inst. Technology

957

Butsyk, Sergey

State University of New York at Stony Brook

PHOBOS

Creighton University

PHENIX

STAR

Byrne, J.

Creighton University

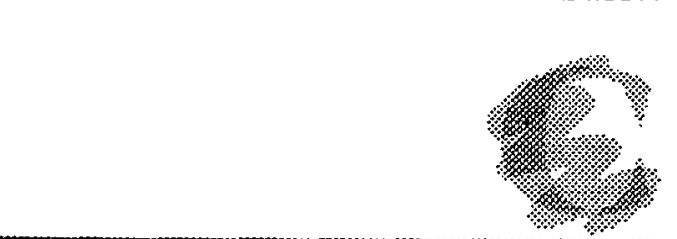

Caccia, $Z$.

Cadman, R.

Caines, $\mathrm{H}$.

Calderon, Manuel

Calen, $\mathrm{H}$.

Camard, Xavier

Cameron, $\mathbf{P}$.

Canfield, P. C.

Carabello, S.

Cardenas, A.

Carey, R. M.

Carman, D.

Carpenter, J. M.

Carroll, A. S.

Carroll, J.

Case, $T$.

Casey, R. M.

Caskey, W.

Cason, N. M.

Castillo, J.

Castro, Maria

Cebra, D.

Cen, C.

Ceretto, Federica

Chaloupka, P.

Chamsoutdinov, $\mathrm{G}$.

Chan, C. S.

Chance, J.

Chang, $\mathrm{H}$.

Chang, J.

Chang, W.

Ghang. Y.

Chäpmian, $M$.

Chisinan, $\mathrm{C}$.
University of Catania

Argonne National Laboratory

Ohio State University

Yale University

University Uppsala

Subatech

Brookhaven National Laboratory

Ames Laboratory

Purdue University

Purdue University

Boston University

896

Carnegie-Mellon University

Argonne National Laboratory

Brookhaven National Laboratory

Lawrence Berkeley Laboratory

Lawrence Berkeley National Laboratory

STAR

STAR

STAR

954, 953

PHENIX

925,880

903

940

STAR

821

906,885

938

PHOBOS, 923, 850

STAR

895

Boston University

952

University of California-Davis

895

852

STAR

STAR

Wayne State University

University of California-Davis

TRIUMF

STAR, 895

State University of New York at Stony Brook 949

Laboratory of High Energy Physics - Dubna

PHENIX

STAR.

Michigan State Univesity

City College of New York

STAR

891

895

PHOBOS

Massachusetts Inst. Technology

University of California-Riverside

PHENIX, 917, 866

University of California-Riverside

National Central University, Taiwan

917

PHOBOS

936,813

Brookhaven National Laboratory 
Chattapadhyay, S.

Chemakin, I.

Chen, A.E.

Chen, D. J.

Chen, $S$.

Chen, Y.

Chen, $Z$.

Chernenko, S.

Cherney, $M$.

Cherry, M. L.

Chertovskikh, A.

Chevel, Andrei

Chi, C. Y.

Chiang, I-H.

Chikanian, A.

Chiu, $M$.

Choi, B.

Choi, Ihn jea

Chrien, R. E.

Christensen, $N$.

Christian, E. R.

Christie, W.

$\mathrm{Chu}, \mathrm{P}$.

Chu, Y. Y.

Chung, $P$.

Chung, S. U.

Cianciolo, $\mathrm{T}$.

Cianciolo, V.

Cianciolo, V.

Cibor, J.

Clajus, M. H.

Claytor, $\mathrm{N}$.

Cleland, W.

Clemen, $M$.

Clement, J. M.

Cobigo, Yann

Coe, S.

Coffin, J-P.

Coghen, $\mathrm{T}$.

Cole, $B$.

Cole, J.

Comfort, J. R.

Conin, $\mathrm{L}$.

Conner, $\mathrm{C}$.

Connor, $\mathrm{D}$.

Conrad, $\mathbf{H}$.

Constantin, F.

Conte, $\mathbf{M}$.

Convertino, A.

Convery, $\mathrm{M}$.

Cooper, P.

Cooper, P.S.

Cooper, R.

Corbo, J.

Corlett, J.
Wayne State University

STAR

Columbia University

National Central Univesity, Taiwan

910,866

PHOBOS

Los Alamos National Laboratory

$947,919,898$

TRIUMF

University of California-LosAngeles

949

STAR

Brookhaven National Laboratory

866

Laboratory of High Energy Physics - Dubna

Creighton University

STAR

STAR

868

Louisiana State University

821

Budker Inst. For Nuclear Physics

Petersburg Nuclear Physics Institute, Russia

Columbia University

PHENIX

Brookhaven National Laboratory

Yale University

Columbia University

University of Texas - Austin

Yonsei University

Brookhaven Natl. Laboratory

University of Auckland

Goddard Space Flight Center

pp2pp, 931, 930, 929,909, 907, 906, 905, 890, 885, 856, 813

Brookhaven National Laboratory

Indiana University IUCF

Brookhaven National Laboratory

State University of New York-Stony Brook

PHENLX, 917, 866

$949,926,787$

STAR, 941

PHENIX

STAR

PHENIX

Brookhaven National Laboratory

850

934

STAR

880

917,866

895

852

Oak Ridge National Laboratory

PHENIX

Lawrence Livermore National Laboratory

910

Massachusetts Institute of Technology

866

Texas A\&M University

University of California-Los Angeles

Lawrence Berkeley Laboratory

University of Pittsburgh

University of Pittsburgh

Rice University

Commissariat A l'Energie Atomique

Yale University

Institute Subatomics de Strasbourg

Inst. Nuclear Physics, Poland

Columbia University

BRAHMS

$927,924,914,913,909,890$

892

877

877

891

PHENLX

864

STAR

PHOBOS

Idaho National Engineering Laboratory

$910,895,866$

877

Arizona State University

SUBATECH-Nantes

$954,953,931,927,914,913,907$

STAR

University of Illinois-Chicago

917

University of California-Irvine

871

Forschungszentrum Jülich

938

University of Bucharest-Romania

University of Genova and Sezione INFN-Italy

BRAFIMS

Massachusetts Inst. Technology

$\mathrm{pp} 2 \mathrm{pp}$

Princeton University

Lawrence Berkeley Laboratory

787

Fermi National Accelerator Laboratory

$957,947,919,898$

Bechtel Nevada

949

945

Brookhaven National Laboratory

PHOBOS

Lawrence Berkeley National Laboratory

951 
Cormier, $\mathrm{T}$.

Cosolino, $\mathrm{M}$.

Costa, S.

Courant, E. D.

Courant, $\mathrm{H}$.

Cowan, T. E.

Cox, A.

Craig, $\mathrm{K}$.

Cramer, John G.

Crawford, $\mathrm{H}$.

Cristie, W.

Crittenden, R. R.

Cronquist, $M$.

Crowe, $\mathrm{K}$.

Cuccinota, $F$.

Cui, $X$.

Cullen, J.

Culpepper, W. X.

Cumming, J. B.

Cummings, J. P.

Cummingg, J. R.

Cushman, P.

Cvema, F.

Cyliax, I.

Czyz, W.
Wayne State University

University of Rome, Thor Vergara

University of Catania

Brookhaven National Laboratory

University of Minnesota

Lawrence Livermore National Laboratory

USAF Armstrong Laboratory

Arizona State University

University of Washington

University of California-Space Science Laboratory

Brookhaven National Laboratory

Indiana University

Space Science Laboratory

Lawrence Berkeley National Laboratory

NASA Johnson Space Center

University of Houston

Brookhaven National Laboratory

National Aeronautics and Space Administration

Brookhaven National Laboratory

Rensselaer Polytechnic Institute

Washington University

University of Minnesota

Los Alamos National Laboratory

Indiana University

Jagellonian University, Poland
STAR, 941, 877, 864

957

896

880

850

933

919

927, 914, 913

STAR

STAR, 917, 896, 866

896

852

896

895

957

931,907

821

922

917,866

852

934

952,821

939

STAR

PHOBOS

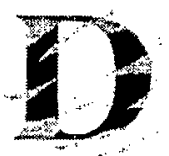

Dabrowski, B.

Instg. Nuclear Physics, Poland

PHOBOS

McGill University

877

Institute of Nuclear Physics-Krakow

868

Brookhaven National Laboratory

821

Indiana University

949

Brookhaven National Laboratory

852

895

877

University of São Paulo

PHENLX

University of São Paulo

877

925

864

885

TRIUMF

906,813

852

$\mathrm{pp} 2 \mathrm{pp}$

University of Texas-Arlington

Brookhaven National Laboratory

University of Illinois

University of California-Irvine

Massachusetts Inst. Technology

University of Minnesota

Rice University

Moscow State University

BRAHMS, 896, 866

$952,924,821$

896,871

PHOBOS

931, 907

STAR

852

STAR

Yale University 
Deng, W.

Deninger, W.

Denisov, S.

DeNolfo, G. A.

Depommier, $P$.

Derevschikov, A. A.

Despet, $\mathrm{M}$.

Deutsch, $M$.

Dhawan, S.

Dhawan, S. K.

Dicello, J.

Didenko, L.

Diebold, G.

Dienes-Jones, $\mathrm{P}$.

Dietel, $\mathrm{T}$.

Dietzsch, O.

Dinneen, $T$.

Diwan, $\mathrm{M}$.

Djikibaev, R. M.

Do, $\mathrm{H}$.

Dominik, W.

Dong, W.

Doornbos, J.

Dorofeev, V. A.

Doskow, J.

Douvernoi, $M$.

Dowd, J.

Drakine, Iouri

Drancourt, C.

Draper, B.

Draper, J.

Drigert, $M$.

Druzhinin, V.P.

Du, Fei

Dubinina, A. I.

Dudkin, G. N.

Dunin, $\mathrm{V}$.

Dunlop, $\mathrm{J}$.

Dunlop, James

Duong, L.

Durum, Arthur

Durant, S.

Durante, $M$.

Dushkin, A.

Dzierba, A. R.
Kent State University

University of Illinois

STAR

821

Institute for High Energy Physics-Protvino

Caltech

University of Montreal

Institute for High Energy Physics-Protvino

Inst. Nuclear Physics, Poland

Massachusetts Inst. of Technology

Yale University

Yale University

John Hopkins Medical Inst.

Brookhaven National Laboratory

Yale University

Louisiana State University

University of Frankfurt

University of São Paulo

Lawrence Berkeley Laboratory

Brookhaven National Laboratory

Institute for Nuclear Research

Yale University

Warsaw University, Poland

University of California-LosAngeles

TRIUMF

Inst. For High Energy Physics - Protvino, Russia

Indiana U. Cyclotron Facility

Pennsylvania State University

University of Massachusetts-Dartmouth

Kurchatov Institute, Russia

SUBATECH-Nantes

Abilene Christian University

University of California-Davis

Idaho National Engineering Laboratory

Budker Institute for Nuclear Physics

Yale University

Institute of Theory and Experimental Physics-Moscow

Tomsk Nuclear Physics Institute-Russia

Laboratory of High Energy Physics - Dubna

Massachusetts Institute of Technology

Yale University

University of Minnesota

Inst. for High Energy Pysics, Russia

Tel-Aviv University

University "Federico II",Napoli

Institute for High Energy Physics-Protvino

Indiana University
PHENIX, 852

934

936

STAR, 925

PHOBOS

820

9.26

865,821

957, 947

STAR

813

868

STAR

PHENIX, 877

892

$949,923,787$

940

865

STAR

STAR

885

852

950

934

852

PHENIX

STAR

914, 913

STAR, 895

877

821

STAR

868

pp2pp

STAR

917, 866

STAR

821

PHENIX

850

957

852

852

Earl, D. D.

Oak Ridge National Laboratory

Earle, W.

Boston University

Eckardt, V.

Max-Planck-Institute fuer Physik 
Eckhause, M.

Ecklund, K.

Edwards, C.

Efendiev, A.

Efimov, L.

Efstathiasdis, E.

Efstathiasdis, E.

Egger, J.

Egorov, O. K.

Eilerts, $S$.

Eiseman, S. E.

Elaasar, M.

Eldredge, $\mathrm{W}$.

Ellsworth, J.

Emelianov, V.

Empl, A.

Endo, $\mathrm{K}$.

Engelage, J.

Enokozono, Akitomo

Enyo, $\mathrm{H}$.

Eppley, G.

Erazmus, B.

Erin, S. V.

Esumi, Shin-ichi

Etkin, A.

Eugenio, P.

Evans, $\mathrm{H}$.

Ewell, L.
College of William and Mary

$935,924,871$

Stanford University

871

Los Alamos National Laboratory

931,907

JINR-Dubna

Laboratory of High Energy Physics - Dubna

Boston University

821

891

865

868

950,865

891

923

917,866

PHOBOS

STAR

Moscow Engineering Physics Institute

University of Houston

$940,931,929,907,905$
821
STAR $917,896,866$

KEK

Space Science Laboratory

Hiroshima University

University of Kyoto

Rice University

$940,931,929,907,905$
821
STAR $917,896,866$

STAR, 917, 896, 866

PHENIX

813

SUBATECH-Nantes

STAR

STAR

925

Institute for High Energy Physics-Protvino

PHENIX

891

Brookhaven National Laboratory

906,852

Carnegie-Mellon University

Case Western Reserve University

Iowa State University
Fachini, $P$.

Fachini, $P$.

Fadem, B.

Faine, $V$.

Fanebust, K.

Farley, F. J. M.

Fedotovich, G. V.

Feinberg, B.

Felder, C.

Felea, D.

Ferdousi, Tahsina

Ferenc, D.

Fernow, R.

Fessler, $\mathrm{H}$.

Fickinger, W.

Fields, D. E.

Filimonov, $\mathrm{K}$.

Finch, E.

Finnemore, D. K.

Fischer, $\mathrm{H}$.
Brookhaven National Laboratory

Wayne State University

STAR

941

941

Iowa State University

Brookhaven National Laboratory

STAR

Fysisk Insitute-Norway

Yale Unviersity

BRAHMS

952, 821

821

892

865

Lawrence Berkeley Laboratory

University of Pittsburgh

University of Bucharest-Romania

University of California - Riverside

University of California - Davis

Brookhaven National Laboratory

Max-Planck-Institute fuer Physik

Case Western Reserve University

University of New Mexico

Lawrence Berkeley Laboratory

Yale University

Ames Laboratory

University of Freiburg
BRAHMS

PHENIX

STAR

951, 910

STAR

856,811

950,926

PHENIX, STAR

STAR, 941

903

$906,885,813$ 
Fischer, $\mathrm{H}$.

Fisyak, Y.

Fitch, J.

Fliere, D.

Flores, $\mathrm{M}$.

Foley, K. J.

Fokin, Sergey

Foster, C. C.

Fouladi, B.

Frank, J. S.

Franklin, G. B.

Frantz, Justin

Franz, J.

Frawley, A. D.

Frehaut, J.

French, A.

Friedl, $M$.

Fu, Jinghua

Fujino, D.

Fujiwara, $\mathrm{T}$.

Fukuda, T.

Fukui, Y.

Fung, S.-Y.

Furic, $M$.

Furusawa, Y.
University of New Mexico

Brookhaven National Laboratory

Massachusetts Inst. Technology

University of Frankfurt

Space Science Laboratory

Brookhaven National Laboratory

Kurchatov Institute, Russia

Indiana University Cyclotron Facility

Texas A\&M University

Brookhaven National Laboratory

Carnegie-Mellon University

Columbia University

University of Freiburg

Florida State University

CEA

Wayne State University

Massachusetts Inst. Technology

Inst. Particle Physics - China

Lawrence Livermore National Laboratory

Kyoto University

INS-University of Tokyo

Lawrence Berkeley Laboratory

University of California-Riverside

University of Zagreb

Natl. Inst. Of Radiological Sciences, Japan
906,865

STAR

PHOBOS

STAR

896

STAR, 891

PHENIX

904

957

$949,923,787$

931, 924, 907, 906, 885, 813

PHENIX

$906,885,813$

PHENIX, 910

939

877

PHOBOS

STAR

933

949

$930,929,906,905$

951

917,866

931,907

919
Gaardhoje, J. J.

Gabriel, K.

Gabriel, T. A.

Gaertner, A.

Gagliardi, C.

Gagunashvili, N.

Gall, K. P.

Gallardo, J.C.

Galuszka, K.

Gan, L.

Gang, $F$.

Gans, Jon

Ganz, R.

Garcia, E.

Garcia-Solis, E.

Gardner, C.

Gardner, R.

Garrard, T. L.

Gasser, J.

Gatley, J.

Gaudichet, L.

Gauland, C.

Gazdzicki, M.

Geer, L. Y.
Niels Bohr Inst. for Astronomy, Physics and Geophysics-U.Copenhagen

Pennsylvania State University

Oak Ridge National Laboratory

Max-Planck-Institute fuer Physik

Texas A\&M University

Laboratory of High Energy Physics - Dubna

Boston University

Brookhaven National Laboratory

Institute Nuclear Physics, Poland

Hampton Univesity

Wayne State University

Yale University

University of Illinois-Chicago

University of Maryland

University of Mlinois-Chicago

Brookhaven National Laboratory

Indiana University

Caltech

University of Bern

Brookhaven National Laboratory

SUBATECH-Nantes.

Arizona State University

University of Frankfurt

Washington University
BRAHMS

850

951

STAR

STAR

STAR

811

951

PHOBOS

$906,885,813$

906,877

STAR

917

917,866

PHOBOS

821

852

934,869

865

919

STAR

931, 907

STAR

869 
Gelderloos, C. J.

Geller, J.

George, N.

George N.

Gerald, J.

Germain, $M$.

Gerwitz, J.

Geurts, F.

Ghazikhanian, V.

Ghosh, Tarun

Giacomich, R.

Gibson, A.

Gibson, B. F.

Giles, B.

Gilkes, $M$.

Gilkes, M.

Gill, $\mathbb{D}$.

Gill, $\mathbb{R}$.

Gillitzer, A.

Gimeno-Nogues, F.

Giron, S.

Glass, G.

Glavanakov, I. V.

Glenn, Andrew

Glenn, J. W.

Gninenko, S. N.

Godlewsi, J.

Gojak, C.

Golubev, V. B.

Gomes, C.

Goncharenko, Yu. M.

Gonzalez, J.

Gordeev, A.

Goto, Y.

Gould, $\mathrm{H}$.

Grabski, J.

Grachov, Oleg

Graessle, S.

Gray, F.

Green, L.

Green, M. A.

Greene, G.

Greenc, V. S.

Greenwood, D.

Greiner, L.

Greiner, L.

Grene, V.

Gribushin, A. M.

Grigoriev, D. N.

Grigoriev, M.

Grigoriev, V.

Grosnick, D.

Grosse-Perke

Grossmann, A.

Grotowski, K.
University of Colorado

Brookhaven National Laboratory

821

Brookhaven National Laboratory

PHOBOS

Yale University

941,864

Brookhaven National Laboratory

931, 907

Institute Subatomics de Strasbourg

STAR

University Pennsylvania

957

Rice University

STAR

University of California-Los Angeles

R, 925

Vanderbilt University

University of Trieste and Sezione INFN-Italy

Valparaiso University

HENDX

$\mathrm{pp} 2 \mathrm{pp}$

Los Alamos National Laboratory

University of Pittsburgh

914,913

931,907

865

Purdue University

895

State University of New York-Stony Brook

910

TRIUMF

813

Brookhaven National Laboratory

pp2pp

Argonne National Laboratory

917

Texas A\&M University

900

University of Minnesota

821

University of Texas at Austin

Tomsk Nuclear Physics Institute-Russia

931,907

$\mathrm{pp} 2 \mathrm{pp}$

University of Tennessee

PHENLX

Brookhaven National Laboratory

Inst. for Nuclear Reserach, Academy of Sciences of Russia

$952,926,880$

865

Institute Nuclear Physics, Geneva

Institute Subatomics de Strasbourg

PHOBOS

STAR

821

Budker Inst. For Nuclear Physics

Massachusetts Inst. Technology

PHOBOS

925

University of California-LosAngeles

STAR

923

Brookhaven National Laboratory

950,925

Lawrence Berkeley Laboratory

892

Warsaw University, Poland

STAR

STAR

$\begin{array}{lr}\text { University of Texas-Austin } & 871 \\ \text { University of } & 821\end{array}$

University of Illinois.

957

Loma Linda University

951,821

939

864

923

896

896

941

852

821

926

STAR

Moscow Engineering Physics Institute

Valpariso University

$954,953,927,924,914,913$

821

821

University of Heidelberg

Jagellonian University-Poland

BRAHMS 
Grover, Andrew

Gruner, R.

Guedon, $M$.

Guilloux, G.

Gulbrandsen, K.

Gumplinger, $P$.

Gunter, J.

Guryn, W.

Gushin, E.

Gushue, S.

Gutbrod, $\mathrm{H}$.

Gutierrez, T.
State University of New York at Stony Brook

Fysisk Institute-Norway

Institute Subatomics de Strasbourg

SUBATECH-Nantes

Massachusetts Inst. Technology

TRIUMF

Indiana University

Brookhaven National Laboratory

Moscow Engineering Physics Institute

Brookhaven National Laboratory

SUBATECH-Nantes

University of California - Davis
PHENIX

BRAHMS

STAR

STAR

PHOBOS

926

852

pp2pp

STAR

PHOBOS , 910, 909, 900, 895, 866, 850

STAR

STAR
Hachiya, Takashi

Hackenburg, R. W.

Haeberlen, U.

Hafen, E. S.

Hagel, $\mathrm{K}$.

Haggerty, J. S.

Haguenauer, $M$.

Haines, $\mathrm{J}$.

Halik, J.

Hall, J.

Halliwell, $\mathrm{C}$.

Hallman, $T$.

Hamagaki, $H$.

Hamblen, J.

Hamilton, S.

Hanabata, $M$.

Hancock, D.

Hansen, Allan

Hansen, A. G.

Hansen, $\mathbf{O}$.

Hanson, A. L.

Hardtke, D.

Hare, M.

Haridas, $P$.

Harper, Greg

Harris, John

Hartman, C.

Hartouni, E. P.

Hashimoto, $\mathbf{O}$.

Hasinoff, $M$.

Hassanein, A.

Hastings, J. B.

Hatcher, N.

Haversten, $K$.

Hayakawa, $\mathrm{H}$.

Hayano, R. S.
Hiroshima University

Brookhaven National Laboratory

PHENIX

Max Planck Institut for Physik

$891,871,852$

821

864

Texas A\&M University

Brookhaven National Laboratory

Ecole Polytechnique-France

Oak Ridge National Laboratory

Institute Nuclear Physics, Poland

Wayne State University

University of Illinois - Chicago

Brookhaven National Laboratory

Institute for Nuclear Study-University ofTokyo

University of Rochester

University of Texas-Austin

Gifu University

College of William and Mary

Los Alamos National Laboratory

Niels Bohr Inst. for Astronomy, Physics and Geophysics-U.Copenhagen

Niels Bohr Inst. for Astronomy, Physics and Geophysics-U.Copenhagen

Brookhaven National Laboratory

Lawrence Berkeley Laboratory

Boston University

Massachusetts Institute of Technology

University of Washington

Yale University

Stanford University

Lawrence Livermore National Laboratory

Tohoku University

University of British Columbia - TRIUMF

Argonne Natinal Laboratory

Brookhaven National Laboratory

Creighton University

Iowa State University

Osaka University

University of Tokyo
BRAHMS

949,787

pp2pp

951,938

PHOBOS

877, 864

PHOBOS

STAR, 896

PHENIX, 917, 866

PHOBOS

871

906

935,871

PHENLX

BRAHMS

BRAHMS

939

STAR

821

941,864

STAR

STAR

871

933

$931,930,907$

$936,926,811$

951

951, 938

STAR

864

929
$917,905,866$ 
Hayoshi, N.

Hayek, $M$.

Hazama, R.

Hazen, E. S.

$\mathrm{He}$, Xiaochun

He, Y. D.

Hebert, $\mathbf{M}$.

Hecox, Ryan

Heering, A.

Heffner, M.

Heffner, Michael

Hei, T.K.

Heintzelman, G.

Heiselberg, $\mathrm{H}$.

Held, Sanuel

Hemnick, T. K.

Henderson, $C$.

Henning, W.

Henry, G.

Henry, $T$.

Heppelmann, S.

Herrmann, $N$.

Hern, K.

Herold, W. D.

Herston, $T$.

Hertzog, D. W.

Hesy, N.

Heuser, Johann

Hicks, D.F.S.

Hicks, $\mathrm{K}$.

Hiebert, R. C.

Hiejima, $\mathrm{H}$.

Hill, J. C.

Hill, $R$.

Hink, P. L.

Hippolyte, B.

Hiramatsu, S.

Hirabayashi, $\mathrm{H}$.

Hirano, Taichi

Hliroyoshi, Hiejima

Hirsch, A. S.

H.jort, E.

Hjort, E.

Hobbs, Robert

Hoff, $C$.

Hoffman, W.

Hoffmann, G.

Hofman, D.

Hogan, $G$.

Hogan, $M$.

Hollis, R.S.

Holm, A.

Holm, C.

Holme, A. K.

Holtzman, B.

\section{RIKEN}

950,925

University of Massachusetts-Dartmouth

852

Osaka University

929

Boston University

821

Georgia State University

PHENIX

University of California-Berkeley

916,903

Stanford University

871

State University of New York at Stony Brook

PHENIX

University of Minnesota

952

University of California-Davis

Lawrence Livermore National Laboratory

895

Columbia University

PHENIX

Massachusetts Institute of Technology

$957,947,919,898$

917,866

Niels Bohr Inst. for Astronomy, Physics and Geophysics-U.Copenhagen

University of Tennessee

SUNY at Stony Brook

BRAHMS

PHENIX

Massachusetts Inst. Technology

877

Argonne National Laboratory

PHOBOS

Lawrence Livermore National Laboratory

Texas A\&M University

Pennsylvania State University

917

939

STAR

GSI-Darmstadt

STAR, 850

College of William and Mary

877

935

865

Paul Scherrer Institute

STAR

Purdue University

University of Illinois

24,821

University of Birmingham

State University of New York at Stony Brook

811

Brookhaven National Laboratory

Ohio State University

PHENIX

PHOBOS

Texas A\&M University

820

835

Columbia University

910

Iowa State University

Argonne National Laboratory

941,864

STAR

934

Washington University in St. Louis

STAR

Institute Subatomics de Strasbourg

880

KEK

821

University of Tsukuba

PHENIX

Columbia University

910

Purdue University

Lawrence Berkeley Laboratory

STAR, 941, 895. 864

STAR

895

Purdue University

PHENIX

871

College of William and Mary

829

University of Texas

University of Texas

STAR, 896, 871

Argonne National Laboratory

PHOBOS, 917

933, 920

Los Alamos National Laboratory

PHOBOS

Massachusetts Inst. Technology

PHOBOS

University of Illinois - Chicago

BRAHMS

BRAHMS

Niels Bohr Inst. for Astronomy, Physics and Geophysics-U.Copenhagen

University of Oslo-Norway

BRAHMS

University of Illinois-Chicago 
Holynski, R.

Holzman, B.

Holzmann, Wolf

Homma, Kensuke

Homma, S.

Hong, $B$.

Hoover, Andrew

Horsley, Mathew

Horvath, D.

Hotchi, $\mathrm{H}$.

Hoversten, R.

Howe, Mark A.

Hseuh, H-C.

Hseuh, S.

Hsi, W-Ch

$\mathrm{Hu}, \mathrm{J}$.

$\mathrm{Hu}$, Yuan

Huang, $\mathrm{H}$.

Huang, $\mathrm{H}$.

Huddleston, $\mathbf{J}$.

Hughes, B. J.

Hughes, $V$.

Humanic, $T$.

Hungerford, E. V.

Hunt, W.

Hunter, J.

Huo, L.

Huso, D.

Hutter, Richard
Institute of Nuclear Physics-Krakow

Brookhaven National Laboratory

State University of New York at Stony Brook

Hiroshima University

Institute for Nuclear Study-University of Tokyo

SUNY at Stony Brook

New Mexico State University

Yale University

Central Research Inst. For Physics, Budapest

University of Tokyo

Iowa State University

University of Washington

Brookhaven National Laboratory

Fermilab

Indiana University

TRIUMF

Inst. Particle Physics - China

Brookhaven National Laboratory

University of California-Los Angeles

Abilene Christian University

Boston University

Yale University

Ohio State University

University of Houston

Indiana University

Lawrence Berkeley Laboratory

Harbin University

NSBRI-John Hopkins Medical Inst.

State University of New York at Stony Brook
PHOBOS, 868

PHOBOS

PHENIX

PHENIX

$9,17,866$

877

PHENIX

STAR

811

906

941

STAR

821

880

900

949

STAR

$950,925,880$

STAR, 941, 925, 896, 864

914,913

952,821

821

STAR, 896

$940,931,929,907,905$

STAR

STAR

895

957

PHENIX

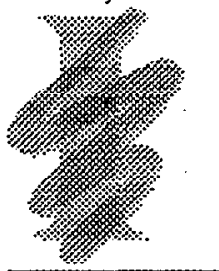

Ibeda, $\mathbf{H}$.

Ichihara, $\mathrm{T}$.

Ichii, S.

High Energy Accelerator Research Org. (KEK)

RIKEN

KEK

Kyoto University

Ichikawa, A.

University of California-Los Angeles

821

Igo, G.

University of Kyoto

Iijima, $T$.

limina, $T$.

KEK

906,885

Ikeda, $\mathrm{H}$.

KEK

Ikonnikov, Victor

Kurchatov Institute, Russia

Imai, $\mathrm{K}$.

Kyoto University

Imazato, $J$.

Inagaki, $T$.

Ingrassia, $\mathbf{P}$.

Insolia, A.

KEK, National Laboratory for High Energy Physics

KEK, National Laboratory for High Energy Physics

STAR, 925,896
813,811

813,811

885
938

PIIENIX

Brookhaven National Laboratory

University of Catania

Iordanova, A.

University of Illinois - Chicago

$950,906,885,813$

949,787

Stanford University

880

Irwin, G. M.

Washington University

Israel, M. H.

Abilene Christian University

PHOBOS

871

934

University of Riken 
University of Kansas

BRAHMS

Ito, $\mathrm{M}$.

Thomas Jefferson National Accelerator Facility

Princeton University

University of Notre Dame

Particle Physics Laboratory - Dubna

Ivanshin, $\mathrm{Y}$. Ivashkin, A.

Institute for Nuclear Research-Moscow

Tokyo Inst. of Technology

Jackson, J.

Jacobs, P.

Jacobs, W. W.

Jaffe, D.E.

Jain, V.

Jang, Woo Young

Janik, $M$.

Jaradat, $\mathrm{H}$.

Jared, R.

Jensen, P.

Jeon, D.

Jia, Jiangyong

Jia, L.

Jipa, A.

Johnson, Austin

Johnson, C.

Johnson, E.

Johnson, I.

Johnson, $\mathrm{K}$.

Johnson, R. R.

Johnson, S.

Johnson, Stephen

Johnston, $\mathrm{K}$.

Johnston, $\mathrm{K}$.

Johnstone, $\mathrm{C}$.

Jones, $P$.

Jones, R. T.

Jones, W. V.

Jorgensen, $T$.

Joseph, J.

Judd, E.

Jundmann, $\mathrm{K}$.

Jundt, $F$.

Jung, Yeondae

Jungmann, $\mathrm{K}$.

Jurak, A.
Brookhaven National Laboratory

Lawrence Berkeley Laboratory

Indiana University

Brookhaven National Labortory

Brookhaven National Laboratory

Korea University

Warsaw University, Poland

Wayne State University

Lawrence Berkeley Laboratory

University of Texas

Indiana University IUCF

State University of New York at Stony Brook

Brookhaven National Laboratory

University of Bucharest-Romania

University of Tennessee

CERN

University of Rochester

Lawrence Berkeley Laboratory

Brookhaven National Laboratory

TRIUMF

SUNY at Stony Brook

Lawrence Livermore National Laboratory

Louisiana Tech University

University of North Carolina

Fermi National Laboratory

University of Birmingham

University of Connecticut

Louisiana State University

Georgetown University Medical Center

Human Nutrition Research Center on Aging

Space Science Laboratory

Physik Inst. der Universit ${ }^{8}$ ot Heidelberg

Institute Subatomics de Strasbourg

Kangnung National University

University of Heidelberg

Institute of Nuclear Physics-Krakow
821

STAR

STAR, 948

949

926.787

PHENIX

STAR

941

STAR

896

880

PHENIX

821

BRAHMS

PHENIX

951

PHOBOS

STAR

788

\section{5}

877

PHENIX

$931,923,907$

905

932

STAR

924

868

919,898

957

STAR, 896, 866

952

STAR

PHENIX

821

868 
Kabana, Sonya

Kabe, S.

Kachaev, I. A.

Kagel, G.

Kahn, S. A.

Kainz, K.

Kale, P.

Kalogeropoulos, $\mathrm{T}$.

Kametani, Soichiro

Kamihara, Noboyuki

Kanavets, V. P.

Kane, J.

Kane, J.L.

Kaneko, HJ.

Kanematsu, N.

Kaneta, $M$.

Kang, J. H.

Kann, Mikhail

Kapinos, P.

Kaplan, M.

Kaplin, V. A.

Karakash, A.

Karavichev, $\mathrm{O}$.

Kaspar, H.

Kaspar, $\mathrm{H}$.

Kasprzyk, T.

Katayama, $\mathrm{T}$.

Kato, Koichi

Katzy, J.

Kawall, D.

Kawamura, M.

Kazantsev, Andrei

Kazumori, $M$.

Keane, D.

Kelly, S.

Kelly, Sean

Kern, B.

Kern, W.

Kettel, S.

Khabibullin, M.M.

Khachaturov, Bakshi

Khan, N.

Khanzadeev, Alexi

Khaustov, P.

Khazin, B. I.

Khodyrev, V.

Khotjanzev, A.
Yale University

KEK, National Laboratory for High Energy Physics

STAR

949,787

Inst. For High Energy Physics - Protvino, Russia

949,852

940,935

951

896

Brookhaven National Laboratory

Rice University

Alabama Agr. And Mech. University

Syracuse University

University of Tokyo

Tokyo Inst. of Technology

Institute for Theoretical and Experimental Physics-Moscow

College of William and Mary

Massachusetts Inst. Technology

Kyoto University

University of California-Irvine

Lawrence Berkeley Laboratory

Yonsei University

Petersburg Nuclear Physics Institute, Russia

TRIUMF

Carnegie-Mellon University

Moscow Engineering Physics Institute

Moscow Engineering Physics Institute

Inst. for Nuclear Research, Moscow

Yale University

Paul Scherrer Institute

Argonne National Laboratory

RIKEN

Waseda University

Massachusetts Inst. Technology

Yale Unviersity

Tokyo Inst. of Technology

Kurchatov Institute, Russia

INS - University of Tokyo

$957,947,919$

856

PHENIX

PHENIX

925

$935,924,871$

PHOBOS

866

871

STAR

917, 910, 866

PHENIX

787

STAR, 896, 895

STAR, pp2pp

STAR, pp2pp

923

926

865

STAR, 914, 913

880

PHENIX

PHOBOS

821

821

PHENIX

787

Kent State University

University of California-Los Angeles

Columbia University

Indiana University

University of Massachusetts-Dartmouth

Brookhaven National Laboratory

Inst. for Nuclear Research

Weizmann Inst. of Science

University of Rochester

Petersburg Nuclear Physics Institute, Russia

Carnegie-Mellon University

Budker Institute for Nuclear Physics

Institute High Energy Physics-Protvino

Institute for Nuclear Research-Moscow
STAR, 910, 895

PHENIX

852

852,818

$949,923,787$

949,926

PHENIX

PHOBOS

PHENIX

885

821

STAR

949, 926 
Kim, B.

Kim, Do Hyun

Kim Dongjo

Kim, E. J.

Kim, E. J.

Kim, Ealgoo

$\mathrm{Kim}$, Hong Joo

Kindem, J.

Kineshita, $\mathrm{K}$.

King, B. J.

King, E.

King, Joshua

King, N. S. P.

Kinnison, J.

Kirchner,D.

Kirk, $\mathrm{H}$.

Kiryluk, J.

Kishi, Y.

Kishimoto, T.

Kisiel, A.

Kitching, $P$.

Kiyomichi, Akio

Kiyoyama, Kouji

Klarmann, J.

Klay, J.

Klein, $S$.

Klein-Boesing, $\mathrm{C}$.

Klyachko, A.

Knecht, N.

Kobayashi, Hideyuki

Kobayashi, M.

Kochetkov, V.

Kodolva, O. L.

Koehler, A.

Koehler, Daniel

Koetke, D. D.

Kohama, Takeshi

Kolganova, E. D.

Kolobashkina, L.

Koltick, D.

Komatsubara, T.

Komisarcik, K.

Komkov, Boris

Konaka, A.

Kondo, Y.

Konisgmann, $\mathrm{K}$.

Konstantinov, A.

Koo, Heon Soo

Kopytine, Mikhail

Koran, $\mathrm{P}$.

Kori, $\mathrm{H}$.

Korotkikh, V. L.

Korteling, R.

Koslenko, N.

Kosonovsky, E.
Wayne State University

877

Myongji University

Yonsei University

Johns Hopkins University

Yonsei University

Seoul National University

Yonsei University

University of Minnesota

PHENIX

PHENIX

BRAHMS

917, 866

PHENIX

PHENIX

821

926

951

Brookkhaven National Laboratory

University of Massachusetts-Dartmouth

852,818

Virginia Poly. Inst. \& State University

PHENLX

Los Alamos National Laboratory .

939,933

NSCORTLBNL-CSU

957

STAR

Brookhaven National Laboratory

University of California-LosAngeles

$951,910,818$

STAR

787

Osaka University

Osaka University

930,929

STAR

Warsaw University, Poland

TRIUMF

949,787

University of Tsukuba

PHENIX

PHENIX

Nagasaki Inst. of Applied Science

869

Lawrence Berkeley Laboratory

STAR

Lawrence Berkeley Laboratory

University of Munster

STAR

Indiana University

PHENIX

STAR

University of Regina

914, 913

PHENIX

RIKEN

949,787

852

852

856

PHENIX

Harvard Cyclotron Laboratory

University of New Mexico

Valparaiso University

Hiroshima University

Institute of Theory and Experimental Physics-Moscow

Moscow Engineering Physics Institute

Purdue University

National Laboratory for High Energy Physics (KEK)

Indiana University

Petersburg Nuclear Physics Institute, Russia

TRIUMF

Kyoto University

University of Freiburg

Institute High Energy Physics-Protvino

Yonsei University

State University of New York at Stony Brook

Carnegie-Mellon University

Osaka University

954, 953, 927, 924, 914, 913

PHENIX

868

STAR

940

949,787

948

PHENIX

$949,926,787$

885

906,885

STAR

PHENLX

PHENIX

906,885

906

852

900

Simon Fraser

914,913

850 
Kostin, M. A.

Kotchenda, L.

Kotchetkov, Dmitri

Kotov, I.

Kotula, J.

Kovalenko, A.

Kozik, T.

Kozjevnikov, A.

Kozlenko, N. G.

Kozlov, Alexandre

Kramer, M. A.

Kraus, D. E.

Krauss, R.

Kravtsov, P.

Krechetov, Y.F.

Kreisler, $M$.

Krienen F.

Krizmanic, J. F.

Krofcheck, D.

Kronenberg, A.

Kronkvist, I.

Kruger, $\mathrm{K}$.

Kruglov, S. P.

Krueger, K.

Krupien, $\mathrm{T}$.

Krylov, V.

Kuang, Y.

Kuberg, C.

Kubota, $\mathrm{K}$.

Kucewicz, W.

Kuczewski, P.

Kudenko, Y.

Kudomi, N.

Kuhn, C.

Kuhn, J.

Kullander, S.

Kulbardis, A.

Kulinich, P.

Kumagai, A.

Kumar, S.

Kume, $\mathrm{K}$.

Kunde, G.

Kuno, Y.

Kunz, C.

Kuo, C.M.

Kupsc, A.

Kuraev, E. A.

Kurashige, $\mathrm{H}$.

Kurepin,A.

Kurglov, N.

Kuriki, $M$.

Kurita, K.

Kurokawa, S.

Kuroki, Yoshiaki

Kutuev, R.
Moscow State University

Moscow Engineering Physics Institute

University of California - Riverside

Ohio State University

Institute Nuclear Physics, Poland

Laboratory of High Energy Physics - Dubna

Jagellonian University-Poland

Inst. for High Energy Physics

Petersburg Nuclear Physics Institute

Weizmann Inst. of Science

City College of New York

STAR

PHENIX

896

PHOBOS

STAR

BRAHMS

949

$954,953,924,909,890$

PHENIX

STAR, 891

University of Pittsburgh

865

820

Texas A\&M University

STAR

Moscow Engineering Physics Institute

Tomsk Nuclear Physics Institute-Russia

Lawrence Livermore National Laboratory

pp2pp

910

821

Boston University

Goddard Space Flight Center

University of Auckland

Lawrence Berkeley National Laboratory

University of Minnesota

934

895

$957,947,919,898$

952,821

880

Argonne National Laboratory

Petersburg Nuclear Physics Institute

Argonne National Laboratory

Brookhaven National Laboratory

Joint Inst. Nuclear Research - Moscow

College of William and Mary

Los Alamos National Laboratory

INS-University of Tokyo

University Illinois - Chicago

Brookhaven National Laboratory

Institute for Nuclear Research-Moscow

Osaka University

Institute Subatomics de Strasbourg

Rensselaer Polytechnic Institute

$3,909,890$

STAR, 925

STAR

PHOBOS

935,871

PHENIX

906, 905

PHOBOS

STAR

949,926

929

STAR

852

954,953

Petersburg Nuclear Physics Inst.-Gatchina

Massachusetts Inst. Technology

$954,953,914,913$

PHOBOS

866

896

929

Yale University

Osaka University

STAR, 896

787

KEK, National Laboratory for High Energy Physics

STAR

Carnegie Mellon University

National Central University - Taiwan

University Uppsala

PHOBOS

954,953

Budker Inst. For Nuclear Physics

821

926

Kyoto University

INR - Russia

Moscow State University

906

852

787

National Laboratory for High Energy Physics (KEK)

University of Tsukuba/RIKEN

$950,910,866$

KEK

821

University of Tsukuba

PHENIX

Particle Physics Laboratory - Dubna 
Kuznetsov, A.

Kwiatkowski, K.

Kwon, Y.

Kwon, $Y$.

Kycia, T.F.
Laboratory of High Energy Physics - Dubna

Indiana University

SUNY at Stony Brook

Yonsei University

Brookhaven National Laboratory
STAR

950,900

877

PHENIX, 917

$927,924,914,913,787$
Lacasse, $\mathbb{R}$.

Lacey, $\mathbf{R}$.

Lahab, $\mathrm{K}$.

Lainis, $T$.

Lajoie, $\mathrm{J}$.

Lajoie, $J$.

Lakehal-Ayat, L.

Lamas-Velverde, J.

Lamler, $M$.

Lamont, $\mathbf{M}$.

Lan, $A$.

Lan, K. J.

Landgraf, J.

Landry, $M$.

Landsberg, $\mathrm{L}$.

Lang, $\mathbb{K}$.

Lange, $S$.

Lansdell, C.

Larsen, $\mathbf{R}$.

Larsen, $\mathrm{R}$.

Larson, K. D.

Lane, F.

Lasiuk, Brian

Lauret, J.

Lawrence, $\mathrm{L}$.

Lazarus, D.

Lebedev, A.

Lebedev, Alexandre

Lebedev, V.

Lebrun, $\mathbf{P}$.

Leckey, Sean

LeCompte, $T$.

Lednitski, $\mathrm{R}$.

Lee, J. H.

ILe, $\mathbf{L}$.

Lee, $R$.

Lee, S. Y.

Lee, Y. K.

Lee, Y. Y.

Lefeure, $F$.

Lefort, $\mathbb{T}$.

Leipuner, $\mathrm{L}$.

Leitch, M. H.

Leksanov, A.
McGill University

State University of New York-Stony Brook

Wayne State University

United States Military Academy at West Point

Yale University

Iowa State University

SUBATECH-Nantes

Rice University

Institute Nuclear Physics, Poland

University of Birmingham

University of Houston

University of Houston

Brookhaven National Laboratory

University of Manitoba

Inst. for High Energy Physics

University of Texas-Austin

University of Frankfurt

University of Texas - Austin

Brookhaven National Laboratory

Yale University

University of New Mexico

Brookhaven National Laboratory

Yale University

Brookhaven National Laboratory

Brookhaven National Laboratory

Brookhaven National Laboratory

Brookhaven National Laboratory

Iowa State University

Inst. For Nuclear Research, Academy of Sciences of Russia

Fermi National Laboratory

State University of New York at Stony Brook

Argonne National Laboratory

Laboratory of High Energy Physics - Dubna

Brookhaven National Laboratory

University of Manitoba

University of California-Irvine

Indiana University

Johns Hopkins University

Brookhaven National Laboratory

SUBATECH-Nantes

Indiana University

Brookhaven National Laboratory

Los Alamos National Laboratory

Pennsylvania State University

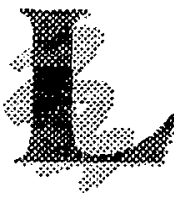

877

895

877

941

864

PHENIX, 941

STAR

STAR

PHOBOS

STAR

931, 929, 907

940

STAR

$906,885,813$

949

871

STAR

STAR

821

923

811

STAR

STAR

STAR

932

865,926

STAR

PHENIX

865

951

PHENIX

925

STAR

BRAHMS, 866

$929,906,885,813$

$940,935,871$

$925,880,864$

BRAHMS

$952,880,821$

STAR

900

865,923

890

850 
Lemoal, C.

Leonhardt, W.

Leontiev, V.

Leray, $S$.

Leske, R. A.

Lesovoy, $\mathrm{O}$.

Leszcznski, P.

Lett, J.T.

Levchenko, A.S.

LeVine, $M$.

Lewis, B.

Lewis, R. A.

$\mathrm{Li}, \mathrm{J}$.

Li, K. K.

$\mathrm{Li}, \mathbf{Q}$.

$\mathrm{Li}, \mathrm{R}$.

Li, X.

$\mathrm{Li}$, Zhiming

Libby, $B$.

Lichtenstadt, J.

Liljestrand, R.

Lim, Hansang

Lin, W.T.

Lindenbaum, $S$.

Lindenbaum, $\mathbf{S}$.

Lindenbusch, $\mathbf{R}$.

Lindgren, A.L.

Lindstrom, $P$.

Lipaev, V.

Lisa, $M$.

Littenberg, L. S.

Liu, Feng

Liu, $\mathrm{H}$.

Liu, $\mathrm{H}$.

Liu, $\mathrm{J}$.

Liu, Lianshou

Liu, Ming

Liu, Zhitu

Liu, Qingjun

Liu, $T$.

Liu, Y. M.

Liu, W.

Liu, Zhitu

Ljubicic, T.

Llope, W.

Lobashev, V. M.

LoCurto, G.

LoCurto, G.

LoDestro, V.

Logashenko, I.

Lolos, G. J.

Long, $\mathrm{H}$.

Longacre, R. S.

Lopatin, I.

Lopez, A.
SUBATECH-Nantes

Brookhaven National Laboratory

Institute High Energy Physics-Protvino

LSN-CNRS

Caltech

Michigan State Univesity

Warsaw University, Poland

Colorado State University

Inst. for Nuclear Research

Brookhaven National Laboratory

University of New Mexico

STAR

STAR

STAR

939

934

STAR

STAR

898

949

STAR, 866

950,949

Penn State University

864

STAR

Institute High Energy Physics - Beijing

$949,926,787$

Wayne State University

Wayne State University

STAR, 877

941

949

STAR

Inst. Particle Physics - China

864

835

Tel-Aviv University

939,933

PHENIX

State University of New York at Stony Brook

PHOBOS

National Central University - Taiwan

Brookhaven National Laboratory and City College of New York

City College of New York

891

STAR

Indiana University

852

898

896

852

STAR, 895

Ohio State University

Brookhaven National Laboratory

Inst. Particle Physics - China

Kent State University

STAR

895

University of California-Riverside

866

University of Houston

904

Inst. Particle Physics - China

Los Alamos National Laboratory

Inst. Particle Physics - China

University of Washington

University of California-Irvine

Harbin University

$949,926,923,787$

Yale University

STAR

PHENIX

STAR

STAR

940,935

895

821

STAR

Inst. Particle Physics - China

Brookhaven National Laboratory

STAR, 896

STAR, 896

940

STAR

896

951

821

Brookhaven National Laboratory

Budker Inst. For Nuclear Physics

$954,927,924,914,913$

STAR, 941

STAR, 896, 891

Brookhaven National Laboratory

Petersburgh Nuclear Physics Institute

Creighton University 
Love, W. A.

Brookhaven National Laboratory

STAR, 896, 891

University of Oslo-Norway

BRAHMS

Lowe, J.

University of New Mexico

University of Brimingham

$949,926,906,865$

Lowe, J.

University Connecticut

813,811

Indiana U. Cyclotron Facility

Lozowski, B.

Joseph Henry Laboratories, Princeton U.

950

$\mathrm{Lu}, \mathrm{C}$.

$\mathrm{Lu}, \mathrm{J}$.

Institute High Energy Physics - Beijing

951

Luccio, A.

Brookhaven National Laboratory

STAR

Ludlam, $T$.

Brookhaven National Labortory

925,880

Lupton, J.

Lutz, J-R.

Texas A\&M University

STAR, 877

Institute Subatomics de Strasbourg

Institute Subatomics de Strasbourg

STAR

Lutz, M-T.

University of California

STAR

Lutze-Mann, L. H.

Brookhaven National Laboratory

$\mathrm{Ma}, \mathrm{H}$.

Madansky, L.

Macdonald, J.

Maeda, K.

Magahiz, R.

Maguire, $\mathrm{C}$.

Magurno, B.

Mai, T.

Majid, W.

Majka, R. D.

Majka, Z.

Makarenko, T.M.

Makdisi, Y.

Maliszewski, A.

Manak, I. J.

Mane, $\mathrm{V}$.

Manglos, $\mathrm{S}$.

Manko, Vladislav

Manley, D. M.

Manley, $\mathrm{S}$.

Manweiler, R. W.

Marcin, M. R.

Mardor, $\mathrm{I}$.

Mardor, Y.

Margetis, $S$.

Mariam, F.

Mariano, G.

Mark, S. K.

Markert, Christina

Marlow, D. R.

Mao, Yajun
Brookhaven National Laboratory

Johns Hopkins University

TRIUMF

Tohoku University

Carnegie-Mellon University

Vanderbilt University

Brookhaven National Laboratory

University of California - Davis

Yale University

Yale University

Jagellonian University-Poland

Particle Physics Laboratory - Dubna

Brookhaven National Laboratory

Warsaw University, Poland

University of Notre Dame

Brookhaven National Laboratory

SUNY Health Science Center

Kurchatov Institute, Russia

Kent State University

University of Rochester

Valparaiso University

University of Texas-Austin

Tel-Aviv University

Tel-Aviv University

Kent State University

Brookhaven National Laboratory

Colorado State University

McGill University

Yale University

Princeton University

Inst. of Physical and Chemical Research

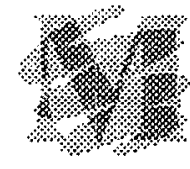

923,865 896

$949,936,926,787$ 929

885,813

941

865

STAR

865

STAR, 941, 864

BRAHIMS

STAR

$950,925,880,850$

STAR

852

932

856

PHENIX

954, 953, 927, 924, 914, 913

PHOBOS

$954,953,927,924,914,913$

871

850

850

STAR

925

919

877

STAR

787 
Marshak, M. L.

Marshall, T.

Martin, L.

Martin, R. D.

Marušic, A.

Marx, J.

Marx, J.

Mashata, $\mathrm{K}$

Masui, Hiroshi

Matathias, Eftychios

Mathews, A. R.

Matis, $\mathrm{H}$.

Matsumoto, Takashi

Matulenko, Yu. A.

Matushevsky, E:

May, $M$.

Mayer, C.

Mayes, B.

Mazavia, D.

McBreen, E.

McCallum, R. W.

McClellan, J. B.

McCorkle, S.

McCrady, R.

McDonald, K.T.

McDonald, S.

McDonough, $\mathrm{J}$.

McGrath, $\mathrm{R}$.

McIntyre, E. K.

McKerley, $M$.

McKinnon, $M$.

McLeod, D.

$\mathrm{McNabb}, \mathrm{R}$.

McPherson, $\mathrm{R}$.

McShane, $T$.

Measday, D. F.

Meier, R.

Meissner, F.

Melnick, Y.

Melnikov, Evgueni

Meng, W-Z.

Menn, W.

Menzel, W.

Merrill, F.

Merzliakov, Yu.

Meschanin, A.

Messer, $M$.

Metting, N. F.

Mewaldt, R. A.

Meyer, C.

Meyers, H. O.

Meyers, P. D.

Meziani, $Z$.

Mi. J.

Miake, Y.
University of Minnesota

Indiana University

SUBATECH-Nantes

College of William and Mary

Ruder Boskovic Institute/U.Calif-Los Angeles

Lawrence Berkeley Laboratory

University of California-Berkeley

KEK

University of Tsukuba

State University of New York at Stony Brook

Los Alamos National Laboratory

Lawrence Berkeley Laboratory

University of Tokyo

Institute for High Energy Physics-Protvino

Laboratory of High Energy Physics - Dubna

Brookhaven National Laboratory

Carnegie-Mellon University

University of Houston

HEP Inst. Tibilsi State University

Brookhaven National Laboratory

Ames Laboratory

Los Alamos National Laboratory

STAR

871

$927,924,914,913,909,890$

STAR

896,891

821

PHENIX

PHENIX

933

STAR

PHENIX

STAR, 925

STAR

$931,930,929,907,906,885$

906

$940,931,929,907$

865

BRAHMS

903

933

877

Brookhaven National Labortory

885,813

951

Joseph Henry Laboratories, Princeton University

University of California-Los Angeles

University of Texas-Austin

890

871

State University of New York-Stony Brook

910,895

Boston University

811

852

Indiana University

STAR

University of California - Davis

University of Illinois-Chicago

University of Minnesota

PHOBOS, 917

Princeton University

821

787

Creighton University

STAR

University of British Columbia

811

Brookhaven National Laboratory

821

Lawrence Berkeley Laboratory

STAR

Institute High Energy Physics-Protvino

STAR

Inst. for High Energy Pysics, Russia

PHENIX

Brookhaven National Laboratory

952,821

University of Siegen

934

865

University of Basel

885,813

Los Alamos National Laboratory

821

STAR

STAR

Institute High Energy Physics-Protvino

Brookhaven National Laboratory

Pacific NW National Laboratory

$947,919,898$

Caltech

934

$924,885,813$

950

787

906

821

PHENIX, 910, 866 
Miachi, T.

Michalon, A.

Michalouski, W.

Michalowski, J.

Mignerey, A.

Mildenberger, J.

Milder, A.

Miller, D.

Miller, J.

Miller, J. P.

Miller, Michael

Miller, $\mathrm{T}$.

Milosevich, Z.

Milov, Alexandre

Minaev, N.

Minami, S.

Mincer, A.

Mineev, $\mathrm{O}$.

Minor, E.

Minor, $\mathrm{R}$.

Mioduszewski, S.

Miskowiec, D.

Mitchell, J.

Mitchell, J. W.

Miyachi, $\mathrm{T}$.

Miyajima, M.

Mizumachi, Y.

Mizuno, Y.

Mogavero, E.

Moinester, M. A.

Moiseenko, V.A.

Mokhov, M. V.

Molzon, W.

Moore, C. F.

Morgan, G. L.

Morgan, T.

Morgan, W.

Mori, Y.

Morikubo, $\mathrm{K}$.

Morimoto, $\mathrm{T}$.

Moriwaki, T.

Morley, K. B.

Morosov, B.

Morozov, V.

Morris, C.

Morrison, D.

Morrison, $\mathrm{T}$.

Morse, W. M.

Moskowitz, B.

Mottershead, C. T.

Moulson, $\mathrm{M}$.

Mueller, $\mathrm{K}$.

Muentz, $\mathbb{C}$.

Muerleilier, M.

Mukhin, V.
INS-University of Tokyo

Institute Subatomics de Strasbourg

STAR

Brookhaven National Laboratory

STAR

Institute Nuclear Physics, Poland

PHOBOS

University of Maryland

TRIUMF

University of Texas-Austin

University of Minnesota

Lawrence Berkeley National Laboratory

Boston University

Yale University

Vanderbilt University

Carnegie-Mellon University

Weizmann Inst. of Science

Institute High Energy Physics-Protvino

Osaka University

New York University

Institute for Nuclear Research

Pennsylvania State University

Lawrence Berkeley Laboratory

University of Tennessee

SUNY at Stony Brook

Brookhaven National Laboratory

NASA-Goddard Space Flight Center

INS-University of Tokyo

Fukui University

Science University of Tokyo

Osaka University

Brookhaven National Laboratory

Tel-Aviv University

Particle Physics Laboratory - Dubna

Fermi National Laboratory

University of California-Irvine

University of Texas at Austin

Los Alamos National Laboratory

Max-Planck-Institute fuer Physik

University of California

KEK

Osaka University

KEK

University of California-Los Angeles

Los Alamos National Laboratory

University Uppsala

Lawrence Berkeley Laboratory

Los Alamos National Laboratory

University of Tennessee

George Washington University

Brookhaven National Laboratory

Brookhaven National Laboratory

Los Alamos National Laboratory

Columbia University

Los Alamos National Laboratory

Brookhaven National Laboratory

University of Houston

Inst. for High Energy Physics
PHOBOS, 917, 866
949,787
871
821

PHOBOS, 917, 866
949,787
871
821

PHOBOS, 917, 866
949,787
871
821

PHOBOS, 917, 866
949,787
871
821

$957,947,919,898$

$952,924,821$

STAR

PHENIX, 941

STAR, 896, 895

PHENIX

STAR

906

940

949,926

850

STAR

910

877

896

934,896

905

949

821

929

896

835

STAR

951

$940,935,871$

STAR, 931, 907

939

STAR

919

880

929

787

$914,913,909.890$

$933,920,900$

954, 953

STAR

933, 931, 920, 907

910

$914,913,909,890$

952,821

866

933

910,866

933,920

866

929

949 
Mukhopadhyay, D.

Mulkey, Z.

Mülmenstädt, J.

Munhoz, M.

Munhoz, M.

Muniruzzaman, $\mathrm{M}$.

Muramatsu, N.

Murnane, J.

Murray, M.

Mutchler, G. S.

Miyachi, $\mathrm{T}$.

Muto, Ryptaro

Myers, Stuart
Weizmann Inst. of Science

Abilene Christian University

Massachusetts Inst. Technology

Wayne State University

Institute de Fisica da University de Sao Paulo

University of California - Riverside

Osaka University - Research Ctr. For Nuclear Physics

Texas A\&M University

Texas A\&M University

Rice University

University of Tokyo

RIKEN

State University of New York at Stony Brook
PHENIX

914,913

PHOBOS

941

STAR

PHENIX

949,787

957

BRAHMS

STAR, 896, 891, 818

906

PHENIX

PHENIX

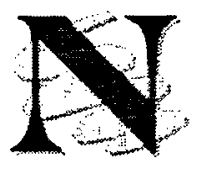

Nachaet, $M$.

Nagae, $T$.

Nagamiya, $S$.

Nagasaka, Yasushi

Nagle, J.

Nagle, $\mathrm{J}$.

Naing, W.

Nakada, Y.

Nakai, K.

Nakamura, $M$.

Nakano, J.

Nakano, $T$.

Namboordiri, M. N.

Nanal, V.

Nandi, Basanta

Napolitano, J.

Narici, L.

Natarajan, $M$.

Natowitz, J.

Naumenko, G. A.

Navon, I.

Nazakawa, K.

Neal, $M$.

Neerman, C.

Nefkens, B.

Nehmeh, S.

Nelson, G.

Nelson, G. A.

Nelson, J.

Nemethy, P.

Nevski, $P$.

Newby, Robert

Newsom, C.

$\mathrm{Ng}, \mathrm{B}$.

$\mathrm{Ng}, \mathrm{C} . \mathrm{F}$.

Nicholson, $\mathrm{H}$.
Wayne State University

KEK

Columbia University

Nagasaki Inst. of Applied Science

Columbia University

Yale University

Hampton University

RIKEN

Science University of Tokyo

Kyoto University

University of Tokyo

Osaka University - Research Ctr. for Nuclear Physics

Lawrence Livermore National Laboratory

Argonne National Laboratory

University of California - Riverside

Rensselaer Polytechnic Institute

University of Rome, Thor Vergara

University of Texas

Texas A\&M University

Tomsk Nuclear Physics Institute-Russia

Tel-Aviv University

Gifu University

Massachusetts Inst. Technology

University of North Carolina

University of California-Los Angeles 954, 953, 931, 927, 924, 914, 913, 909, 907, 890

Wayne State University

Loma Lnda University

Jet Propulsion Laboratory

University of Birmingham

New York University

Brookhaven National Laboratory

University of Tennessee

University of Iowa

Fermi National Laboratory

Brookhaven National Laboratory

Mt. Holyoke College
896

$930,929,906,905$

866

PHENIX

941

864

905

925

936

950

$929,906,905,787$

949

910,866

917

PHENIX

852

957

957,919

BRAHMS

pp2pp

850

906

PHOBOS 905

896

957,919

898

STAR, 813

952, 940

STAR

PHENIX

$\mathrm{pp} 2 \mathrm{pp}$

932

787

850 
Nielsen, B. Svane

Nikitin, V.

Nilsen, B. S.

Nishide, J.

Noble, A.

Nociforo, C.

Nogach, L. V.

Nomachi, $M$.

Nomura, $T$.

Nord, $P$.

Norem, J. $\mathbb{H}$.

Norman, A.

Norman, B.

Normand, $L$.

Nouicer, $\mathrm{R}$.

Noumi, $\mathrm{H}$.

Novakova, $\mathrm{P}$.

Nozar, $M$.

Numao, $T$.

Nurushev, S. B.

Nurushev, S. B.

Nussbaum, T.

Nyanin, Alexander

Nystrand, Joakim
Niels Bohr Inst. for Astronomy, Physics and Geophysics-U.Copenhagen

Particle Physics Laboratory - Dubna

University of Minnesota

Fukui University

University of British Columbia

University of Catania

Institute for High Energy Physics-Protvino

Osaka University

Kyoto University

Valparaiso University

Argonne National Laboratory

College of William and Mary

Kent State University

McGill University

University of Illinois

KEK

Brookhaven National Laboratory

Rensselaer Polytechnic Institute

TRIUMF

Institute for High Energy Physics-Protvino

Moscow Engineering Physics Institute

Rice University

Kurchatov Institute, Russia

Lund University
BRAHMS

STAR

869

949

811

896

STAR, 925

949, 929

949,926

914,913

951,932

935

STAR

877

PHOBOS

930, 929

STAR

852

$949,926,787$

STAR, 950, 925

pp2pp

STAR

PHENIX

PHENIX
Obot, V.

Obraztsov, V.

O'Brien, E.

O'Donnell, J.

Odyniec, G.

Ogawa, A.

Ogilvie, C.

Okada, $\mathrm{K}$.

Okomura, $\mathrm{M}$.

Okorokov, V.

Okumo, $\mathrm{H}$.

Olchanski, K.

Oldenburg, $\mathrm{M}$.

Olness, $J$.

Olshevsky, R.

Olson, D.

Olszewski, A.

Olszewski, A.

Oltchak, A.

Omata, $\mathrm{K}$.

Onderwater, C. J. G.

Onel, Y.

Ono, Masava

Orlov, Y.

Ormes, J. F.

Osborme, L.
Texas Southern University

Inst. for High Energy Physics

Brookhaven National Labortory

Los Alamos National Laboratory

Lawrence Berkeley National Laboratory

Pennsylvania State University

Massachusetts Institute of Technology

Kyoto-Sangyo University

RIKEN

Moscow Engineering Physics Institute

National Laboratory for High Energy Physics (KEK)

Brookhaven National Laboratory

Max-Planck-Institute fuer Physik

Brookhaven National Laboratory

TRIUMF

Lawrence Berkeley National Laboratory

Brookhaven National Laboratory

Institute of Nuclear Physics-Krakow

Moscow Engineering Physics Institute

INS-University of Tokyo

University of Illinois

University of Iowa

University of Tsukuba

Cornell University

Goddard Space Flight Center

Massachusetts Inst. Technology
904

949

877

931,907

STAR, 895

STAR, 850

917,866

813

$950,925,880$

STAR

787

BRAHMS, 852

STAR

BRAHMS, 866

835

STAR, 895

PHOBOS

868

STAR

949,906

952,821

pp2pp

PHENIX

952,821

934

PHOBOS 
Ososkov, G.

Ostrovidov, A. I.

Otomo, A.

Ott, G.

Outa, $\mathrm{H}$.

Overlin, M.

Oyama, K.

Ozben, $\mathrm{C}$.
Laboratory of High Energy Physics - Dubna

Moscow State University

National Laboratory for High Energy Physics (KEK)

University of Texas - Austin

KEK

University of California at Irvine

University of Tokyo

Brookhaven National Laboratory
STAR

852

787

STAR

930, 929, 906, 905

940

PHENLX, 917

821,952
Padley, $\mathbf{P}$.

Paganis, $S$.

Page, $\mathrm{S}$.

Pai, C.

Pak, R.

Pak, R.

Pal, Dipali

Paley, J.

Palmer, R. B.

Pandey, S.

Panebratsev, Y.

Panitkin, S.

Panitkin, S.

Papandreou, $Z$.

Park, H-S.

Park, I.

Parks, D.

Paschke, K.

Passaneau, J.

Patterson, J.

Paul, P.

Pavlinov, A. I.

Pavlinov, Alexi

Pawlak, T.

Peaslee, $D$.

Pedlar, T.

Peng, J-C.

Pentia, $M$.

Penzo, A.

Perepechkin, $Y$.

Pernegger, $\mathrm{H}$.

Peryt, W.

Peterson, G. A.

Peterson, J.

Peterson, R. J.

Petkovic, T.

Petrenko, S.

Petridis, A.

Petrov, V.A.

Petukhov, Y.

Phillips, $\mathrm{S}$.
TRIUMF

University of Texas

University of Manitoba

Brookhaven National Labortory

Brookhaven National Laboratory

University of Rochester

Weizmann Inst. of Science

Boston University

Brookhaven National Laborary

Wayne State University

J.I.N.R.-Dubna

SUNY at Stony Brook

Kent State University

George Washington University/U. Regina

Lawrence Livermore National Laboratory

University of Rochester

University of Houston

Carnegie-Mellon University

Pennsylvania State University

University of Colorado

SUNY at Stony Brook

Institute for High Energy Physics-Protvino

Wayne State University

Warsaw University, Poland

University of Maryland

Northwestern University

Los Alamos National Laboratory

Laboratory of High Energy Physics - Dubna

University of Trieste and Sezione INFN-Italy

INR - Russia

CERN

Warsaw University, Poland

University of Colorado

University of Colorado

University of Colordo

University of Zagreb

Inst.for High Energy Physics

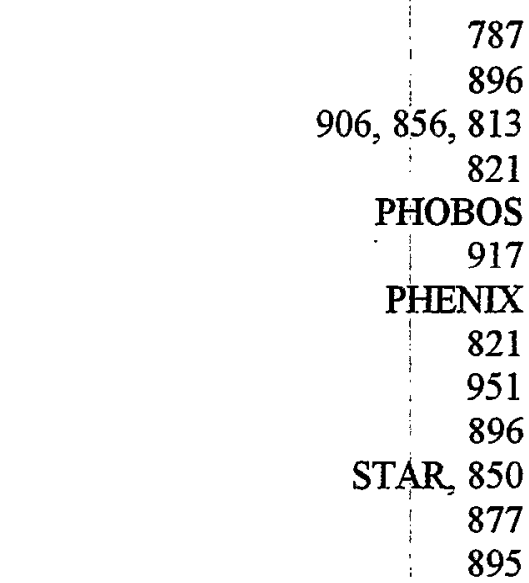

$954,927,924,914,913,909,890$

933

PHOBOS

904

906,885

STAR

$9: 14,913$

877

. 925

STAR

STAR

$954,953,924$

852

$931,907,890$

STAR

pp2pp

906

PHOBOS

STAR

953, 931, 937, 924, 907

914,913

$954,937,927$

931, 907

Iowa State University

949

941

Particle Physics Laboratory - Dubna

STAR

Particle Physics Laboratory - Dubna

STAR

George Washington University 
Piasetzky, E.

Piazza, T.

Pichot, P.

Piekarz, $H$.

Piekarz, $\mathrm{H}$.

Pienkowski, L.

Pile, P. H.

Pinganaud, $W$.

Pinkenburg, $C$.

Pinsky, L.

Pislak, S.

Pislak, S.

Pitt, M.

Planinic, $M$.

Planinic, $M$.

Platner, E. D.

Plesko, M.

Pless, I.

Pluta, J.

Poblaguev, A. A.

Pocanic, D.

Polk. I.

Polly, C.

Polychronakas, V.

Pommot-Maia, $M$.

Pope, J. K.

Pope, Joseph

Poplaguev, A.

Popov, A. V.

Popovic, M.

Porile, N. T.

Poskanzer, A.

Postoev, V.

Potenza, R.

Potrebenikova, E

Potylitsin, A. P.

Poutissou, J-M.

Poutissou, R.

Pozharova, E. A.

Prakhov, S. N.

Prasad, S. C.

Pratt, R.

Prebys, E. J.

Pretz, J.

Price, J. W.

Price, P. B.

Prigl, $\mathbb{R}$.

Prindle, Duncan J.

Prokhavatilov, $\mathbf{M}$.

Prokop, J.

Proskuryakov, A.

Protopopescu, S. D.

Pruneau, C.

Przewoski, B. V.

Pulver, $\mathrm{M}$.
Tel-Aviv University

850

SUNY at Stony Brook

SUBATECH-Nantes

STAR

Brandeis University

829

Flordia State University

820

Warsaw University

Brookhaven National Laboratory

900

SUBATECH-Nantes

State University of New York-Stony Brook

University of Houston

Yale University

University of Zurich

Virginia Polytechnic Institute

Indiana University

University of Zagreb

Rice University

Massachusetts Institute of Technology

Massachusetts lnstitute of Technology

Warsaw University, Poland

Inst. For Nuclear Research, Academy of Sciences of Russia

University Virginia

$931,930,929,924,907,906,885,813$

STAR

895

$940,931,929,907$

926

865

926

STAR

931, 907, 905

STAR, 896, 891

PHOBOS

941,864

STAR

926,865

926

Brookhaven National Laboratory

821

University of Illinois

821

923

Brookhaven National Laboratory

Yeshiva University

949,871

864

University of Tennessee

Inst. for Nuclear Research, Moscow

PHENIX

923

852

932

Fermi National Laboratory

Purdue University

Lawrence Berkeley Laboratory

Inst. For Nuclear Research, Academy of Sciences of Russia

University of Catania

Laboratory of High Energy Physics - Dubna

Tomsk Nuclear Physics Institute-Russia

TRIUMF

TRIUMF

Institute of Theory and Experimental Physics-Moscow

University of California

SUNY Health Science Center

George Washington University

Joseph Henry Laboratories, Princeton University

Yale University

University of California-Los Angeles

University of California-Berkeley

Brookhaven National Laboratory

University of Washington

INR-Russia

George Washington University

Moscow State University

Brookhaven National Laboratory

Wayne State University

Indiana u. Cyclotron Facility

University of California-Los Angeles
STAR, 941, 895, 864

STAR

865

896

STAR

pp2pp

949,787

$949,787,926$

868

954., 953

856

909

951

821

$954,953,890$

916

952,821

STAR

906

909

$923,852,865$

818

STAR, 941, 877, 864

950

914,913 


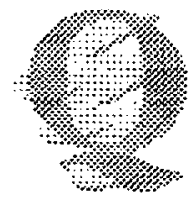

Qian, Z.

Fermi National Laboratory

Quinn, B.

Carnegie-Mellon University

$931,924,907,906,885,813,811$

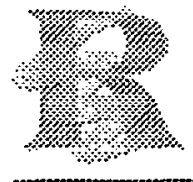

Rabin, B.

Rabin, M. S. Z.

Radomski, S.

Radutsky, G. M.

Rafelski, M.

Rai, G.

Rakness, G.

Ramakrishnan, E.

Ramirez, A.

Ramsay, D.

Ramsberg, E.

Ramsey, W. D.

Rangacharyulu, C.

Ransom, $\mathbf{R}$.

Rasin, V.

Ratner, L.

Ravel, 0 .

Ray, L.

Rbeiz, M.

Read, K.

Rebeck, N.

Reber, E.

Redding, $\mathrm{R}$.

Redin S. I.

Redlinger, G.

Reece, K.

Reed, C.J.

Reed, J.

Rehak, P.

Reichold, D.

Reid, Jeffrey

Reid, J.

Reiley, P.

Reimer, P. E.
University of Maryland Baltimore County

University of Massachusetts

Warsaw University, Poland

Tomsk Nuclear Physics Institute-Russia

Brookhaven National Laboratory

Lawrence Berkeley National Laboratory

Indiana University

Texas A\&M University

Arizona State University

University of Manitoba

Fermi National Accelerator Laboratory

University of Manitoba

University of Saskatchewan

Rutgers University

INR - Russia

Brookhaven National Laboratory

SUBATECH-Nantes

University of Texas - Austin

Massachusetts Inst. Technology

University of Tennessee

Creighton University

Idaho National Engineering Laboratory

Creighton University

Yale University

TRIUMF

Brookhaven National Laboratory

Massachusetts Inst. Technology

Syracuse University

Brookhaven National Laboratory

Creighton University

University of Washington

Vanderbilt University

University of Texas

University of Illinois-Urbana-Champaign
$957,947,919,898$

941,864

STAR

pp2pp

PHOBOS

STAR, 910, 895

STAR

900

914,913

885,813

949

906

936

885

906

925,880

STAR

STAR

PHOBOS

910

STAR

877

STAR

821

$949,926,787$

880

PHOBOS

951,856

865

STAR

STAR

864

943, 856

$-924$ 
Relyea, D. R.

Remsberg, L. P.

Ren, Y.

Renfordt, $\mathbb{R}$.

Renoid, C.

Reshetin, A.

Retiere, F.

Reuter, M.

Reygers, Klaus

Riabov, Victor

Ridiger, A.

Rigalleau, L-M.

Rijssenbeek, $M$.

Riley, P. J.

Rinckel, $\mathrm{T}$.

Rind, 0 .

Riso, Jose

Ritchie, J. L.

Ritchie, S.

Fitter, H. G.

Ritter, J.

R.oberts, B. L.

Roberts, J. B.

Robinson, D. K.

Robmann, P.

Roehrich, D.

Roehrich, D.

Rogachevskiy, $\mathbf{O}$.

Roland, C.

Roland, G.

Romero, J.

Roos, P. G.

Rosati, M.

Rose, A.

Rose, Andrew

Roser, $T$.

Rosenberg: L.

Ross, G.D.

Rössle, R.

Rotondo, F. S.

Rowland, D.

Roy, C.

Roy, J.

Rozon, F.

Rozon, M.

Rozon $\mathrm{M}$.

Ruangma, A.

Rubin, $P$.

Rumbers, Audrey

Runco, M.

Runtzo, M. F.

Runtzo, M. F.

Rusek, A.

Rusek, A.

Russ, D.
Princeton University

Brookhaven National Laboratory

University of Houston

University of Frankfurt

SUBATECH-Nantes

INR - Russia

Lawrence Berkeley Laboratory

University of Illinois - Chicago

University of Munster

Petersburg Nuclear Physics Institute, Russia

Moscow Engineering Physics Institute

SUBATECH-Nantes

State University of New York-Stony Brook

University of Texas-Austin

Indiana U. Cyclotron Facility

Boston University

Wayne State University

University of Texas-Austin

University of California - Davis

Lawrence Berkeley National Laboratory

University of Illinois-Urbana-Chapaign

Boston University

Rice University

Case Western Reserve University

University of Zurich

University of Frankfurt

Fysisk Institute

Laboratory of High Energy Physics - Dubna

Massachusetts Inst. Technology

Massachusetts Inst. Technology

University of California-Davis

University of Maryland

Brookhaven National Laboratory

Vanderbilt University

Wayne State University

Brookhaven National Laboratory

Massachusetts Inst. Technology

Massachusetts Inst. Technology

Freiburg University

Yale University

Texas A\&M University

PHOBOS, 910, 909, 900, 866

904

STAR

STAR

906

STAR

PHOBOS

PHENIX

PHENIX

STAR

STAR

pp2pp

871

950,948

952, 821

STAR

871

STAR

STAR, 895

924

$952,821,811$

STAR, 818

856,811

865

STAR

BRAHMS

STAR

PHOBOS

PHOBOS

STAR, 895

931,907

910,877

941

PHENIX, STAR

$951,950,932,925,880,850$

PHOBOS

PHOBOS

813,811

896,864

900

SUBATECH-Nantes

STAR

787

788

787

813

917

University of Maryland

University of Richmond

935,871

STAR

STAR

950

pp2pp

Moscow Engineering Physics Institute

IHEP

Moscow Engineering Physics Institute

Brookhaven National Laboratory

University of New Mexico

pp2pp, 931, 930, 907, 906, 905, 885

813

Carnegie Mellon University 
Russ, D.

Russell, G.

Russell, J. J.

Russo, G.

Rusek, A.

Rust, D. R.

Ryabchikov, D. I.

Ryan, J.

Rydberg, B.

Rykalin, V.

Rykov, Vladimir

Ryskulov, N.

Ryu, Sangsu
University of Maryland

Los Alamos National Laboratory

University of Massachusetts-Dartmouth

University of Catania

Brookhaven National Laboraory

Indiana University

Inst. For High Energy Physics, Protvino, Russia

Massachusetts Institute of Technology

Lawrence Berkeley Laboratory

Inst. for High Energy Physics

Wayne State University

Budker Institute for Nuclear Physics

Yonsei University

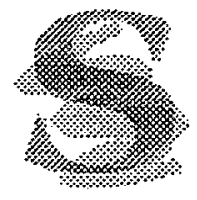

Sachelashvili, T.

Sadler, M.

Sagerer, J.

Saito, N.

Saito, N.

Sajai, E.

Sakaguchi, Takao

Sakai, $\mathrm{H}$.

Sakai, Masashi

Sakamoto, S.

Sakitt, M.

Sako, H.

Sakrejda, I.

Sakuma, Fuminori

Salomon, M.

Salur, Sevil

Sanders, $R$.

Sanders, S. J.

Sandweiss, J.

Sangster, T. C.

Sanita, W.

Sanjari, A.

Sarin, $P$.

Sarracino, J.

Sarycheva, L. I.

Sasao, N.

Sasaki, $T$.

Satinover, Jeffrey

Sato, $\mathbf{R}$.

Sato, $\mathrm{S}$.

Sato, Susumu

Sato, $T$.

Saulys, A.

Savin, I.
HEP Inst. Tibilsi State University

Abilene Christian University

University of Illinois - Chicago

University of Kyoto

RIKEN

Osaka University

University of Tokyo

865

University of Tokyo

Nagasaki Inst. of Applied Science

KEK

Brookhaven National Laboratory

Institute for Nucler Study-University of Tokyo

Lawrence Berkeley Laboratory

Kyoto University

TRIUMF

Yale University

Brookhaven National Laboratory

University of Kansas

Yale University

Lawrence Livermore National Laboratory

University of Rome, Thor Vergara

University of Notre Dame

Massachusetts Inst. Technology

Los Alamos National Laboratory

$927,914,913,909,890$

PHOBOS

813,811

950,925

929

PHENIX

925

PHENIX

821

pp2pp, 856, 811

866

STAR, 896

PHENLX

811

STAR

821

BRAHMS

STAR, 941, 864

910,866

957

852

PHOBOS

933,920

Moscow State University

852

926

787

Osaka University

Yale University

Osaka University

University of Tsukuba

STAR

929

866

PHENIX

Japan Society for the Promotion of Science

KEK, National Laboratory for High Energy Physics

$949,880,821,787$

896,891

Brookhaven National Laboratory

STAR 
Sawada, Shinva

Sawafta, R.

Sawafta, R.

Sawh, $\mathbb{R}-\mathrm{P}$.

Sawicki, P.

Scanlan, R. M.

Schambach, J.

Scharenberg, R. P.

Scheetz, R.

Schindler, S. M.

Schiavon, P.

Schimmerling, W.

Schlei, Bernd

Schmidt, $H$.

Schmidt-Sorensen, J.

Schmitt, H.

Schmitz, N.

Schroeder, L.

Schroeder, L.

Schue, J.

Schuetlauf, A.

Schumacher, R. A.

Schweda, K.

Scott, E.

Sculli, J.

Sedykh, S.

Seger, J.

Sekimoto, M.

Seliverstov, D.

Semenov, V.

Semertzidis, Y.

Sengupta, K.

Sekimoto, $M$.

Semertzidis, Y. K.

Serednyakov, S.

Seth, K.

Setlow, R.

Seto, R.

Seyboth, J.

Seyboth, $P$.

Seyfarth, H.

Seymour, R.J.

Shafi, A.

Shahaliev, E.

Shalnov, A.

Shatunov, Yu. M.

Shaw, Matthew

Shea, J.

Sheen, J.

Shein, I.

Shephard, W. D.

Shestermanov, K.

Shibata, T.

Shigaki, Kenta

Shileev, K.
KEK

North Carolina A\&T University

Brookhaven National Laboratory

PHENIX

University of Houston

Institute Nuclear Physics, Poland

Lawrence Berkeley Laboratory

University of Texas

Purdue University

Brookhaven National Laboratory

Caltech

University of Trieste and Sezione INFN-Italy

NASA Headquarters

Los Alamos National Laboratory

Freiburg University

University of Lund-Sweden

University of Freiburg

Max-Planck-Institute fuer Physik

University of California-Berkeley

Lawrence Berkeley National Laboratory

Creighton University

Max-Planck-Institute fuer Physik

Carnegie-Mellon University

Lawrence Berkeley Laboratory

Indiana University

New York University

University of Illinois

Creighton University

KEK

Moscow Engineering Physics Institute

Inst. for High Energy Physics, Protvino

Brookhaven National Laboratory

Louisiana State University

INS-University of Tokyo

Brookhaven National Laboratory

Budker Institute for Nuclear Physics

Northwestern University

Brookhaven National Laboratory

University of California-Riverside

Max-Planck-Institute fuer Physik

Max-Planck-Institute fuer Physik

Forschungszentrum Jülich

University of Washington

George Washington University

Laboratory of High Energy Physics - Dubna

Moscow Engineering Physics Institute

Budker Institute for Nuclear Physics

Los Alamos National Laboratory

University of Maryland

Wayne State University

Institute for High Energy Physics-Protvino

University of Notre Dame

Institute High Energy Physics-Protvino

Osaka University

University of Tokyo

INR - Russia
$931,930,929,909,907,906,905,885$

$906,890,885,856,813$

904

PHOBOS

951

STAR, 896

STAR, 941, 895, 864

BRAHMS

934

pp2pp

957

PHENIX

885,813

BRAHMS

906

STAR

891

STAR, 895

STAR

STAR

$924,906,885,813$

STAR

852

940

924,821

STAR

906

STAR

923

821

868

906

952

821

924,852

957

917, 866

STAR

STAR

890

STAR

914, 913

STAR

STAR

821

PHENIX

866

896,877

PHENIX, 852

852

STAR

829

PHENIX

906 
Shima, A.

Shimanskiy, S.

Shimizu, Y.

Shimoyama, T.

Shin, B.

Shin, Y. H.

Shina, Toshiyuki

Shinkawa, T.

Shiva Kumar, B.

Shvetsov, V.S.

Shoemaker, F. C.

Sileev, K.

Silin, I.N.

Silvermyr, David

Simon, M.

Simone, G.

Sinden, R. R.

Sinev, N. B.

Skank, $\mathrm{H}$.

Skelly, J. E.

Skoro, G.

Skorotko, T. Yu.

Skulski, W.

Skutnik, Steven

Slaughter, A. J.

Slaughter, D.

Šlaus, I.

Sleege, G.

Smirnitski, V. A.

Smirnoff, N.

Smith, A. J. S.

Smith, B.

Smith, D. L.

Smith, G.

Smith, G. A.

Smith, J.

Smith, P. T.

Smykov, L.

Snead, C. L.

Snellings, R.

Snydstrup, L.

Sobolewski, Z.

Solberg, K.A.

Soldatov, A.

Solodov, E.

Soloviev, L.

Soltz, R.

Soluk, R.

Sonderegger, $\mathrm{P}$.

Sonnadara, U.

Sorensen, P.

Sorensen, S.

Sosin, Z.

Sossong, $\mathrm{M}$.

Soukas, A.
University of Tokyo

957

J.I.N.R. - Dubna

STAR, 850

Osaka University

Fukui University

University of Saskatchewan

Yonsei University

Los Alamos National Laboratory

National Defense Academy of Japan

Yale University

Particle Physics Laboratory - Dubna

Princeton University

INR-Russia

Particle Physics Laboratory - Dubna

Lund University

University of Siegen

National Inst. of Health, Rome, Italy

Texas A\&M University

Moscow State University

906,905

949

936

910

PHENIX

949,787

864

STAR

787

906

STAR

PHENIX

934

957

947

852

Ames Laboratory

941,864

880

Brookhaven National Laboratory

Laboratory of High Energy Physics - Dubna

Institute of Theory and Experimental Physics-Moscow

STAR

868

University of Rochester

PHOBOS

PHENIX

Iowa State University

941,864

939

Lawrence Livermore National Laboratory

Ruder Boskovic Institute

$954,953,927,914,913,909,890$

Ames Laboratory

941,864

Institute of Theory and Experimental Physics-Moscow

868

Yale University

STAR, 896

Princeton University

University of New Mexico

Argonne National Laboratory

TRIUMF

Penn State University

Rensselaer Polytechnic Institute

Indiana University

Laboratory of High Energy Physics - Dubna

Brookhaven National Laboratory

Lawrence Berkeley Laboratory

Brookhaven National Laboratory

Syracuse University

Indiana University

787

950

951

926

864

852

852

STAR

939, 903

STAR

821

856

STAR

852

821

Budker Institute for Nuclear Physics

Institute High Energy Physics-Protvino

STAR

Lawrence Livermore National Laboratory

TRIUMF

CERN

University of Pittsburgh

University of California-LosAngeles

University of Tennessee

Jagellonian University

University of Illlinois

$933,910,866$

787

896

877

STAR

910

BRAHMS

821

Brookhaven National Laboratory 
Sowinski, J.

Sowinski, M.

Spinelli, P.

Spinka, $H$.

Sposato, S. H.

Srivastava, B. K.

St. Claire, L.

Stachel, J.

Stack, K.

Stanislaus, S.

Stankus, Paul

Stanskas, J.

Starinsky, N.

Starostin, A.

Staszel, P.

Staudenmaier, H. M.

Steadman, S. G.

Stearns, R.

Steinberg, P.A.

Steinnaetz, A.

Stepanov, Mikhail

Stephans, G. S. F.

Stephens, D.

Stephenson, E. J.

Stepien, $\mathbb{P}$.

Stevens, A. J.

Stienike, D. L.

Stillman, A.

Stock, R.

Stodulski, M.

Stolpousky, Sasha

Stone, E. C.

Stone, N.

Stone, J. $\mathbb{R}$

Stotzer, R.

Straczek, A.

Strand, R. C.

Streitmatter, R. E.

Strek, IM.

Strikhanov, M. N.

Strikbanov, M. N.

Stringfellow, B. C.

Stroebele, H.

Struck, C.

Suaide, A.

Suda, T.

Sugarbaker, E.

Sugita, Noriyuki

Sugitate, Toru

Sugimoto, S.

Suire, C.

Sulk, Joon Young

Sukaton, R.

Sukhanov, A.

Sulak, L. R.
Indiana University

Institute Nuclear Physics, Poland

STAR, 948

PHOBOS

University of Bari

864

Argonne National Laboratory

Washington University

Purdue University

Lawrence Berkeley Laboratory

SUNY at Stony Brook

Creighton University

Valparaiso University

Oak Ridge National Laboratory

University of Maryland

STAR, 954, 952, 927, 925, 914, 913, 880

STAR, 954, 952, 927, 925, 914, 913, 880

STAR, 895,864

STAR

877

STAR

954, 953, 927, 924, 914, 913

PHENIX

917

877

McGill University

Petersburg Nuclear Physics Institute/UCLA

Jagellonian, University

University of Karlsruhe

Massachusetts Institute of Technology

Vassar College

Brookhaven National Laboratory

Yale University

New Mexico State University

Massachusetts Institute of Technology

NSCORTLBNL-CSU

Indiana Univeristy

Warsaw University, Poland

Brookhaven National Laboratory

University of Notre Dame

Brookhaven National Laboratory

University of Frankfurt

Institute Nuclear Physics, Poland

Wayne State University

Caltech

Lawrence Berkeley National Laboratory

Princeton University

University of New Mexico

Institute Nuclear Physics, Poland

Brookhaven National Laboratory

Goddard Space Flight Center

Institute Nuclear Physics, Poland

IHEP

Moscow Engineering Physics Institute

Purdue University

University of Frankfurt

University of Frankfurt

Institute de Fisica da University de Sao Paulo

Tohoku University

Ohio State University

Hiroshima University

Hiroshima University

INS-University of Tokyo

Institute Subatomics de Strasbourg

Korea University

Carnegie-Mellon University

Brookhaven National Laboratory

Roston University
$954,953,927,924,914,913,890$

BRAHMS

$954,953,927,924,914,913$

866

788,813

PHOBOS

821

PHENIX

PHOBOS, 917,866

957

STAR, 948

STAR

787

852

821

STAR

PHOBOS

STAR

869

895

787

$885,865,813$

PHOBOS

$949,926,787$

934

PHOBOS

950

STAR, pp2pp

STAR, 864

STAR

STAR

STAR

929

STAR, 896

PHENIX

PHENIX

949, 787

STAR

PHENIX

813,811

PHOBOS

821 
Sulanke, T.

Sum, V.

Sumbera, $M$.

Supek, I.

Sutherland, B. M.

Sutter, R.

Sutton, C. S.

Suzuki, Miwako

Švarc, A.

Svirida, D. N.

Symons, J.

Syphers, M.

Szanto de Taledo, A.

Szarska, M.

Szarwas, $P$.

Szymanski, J.
Indiana University

University of Manitoba

Laboratory of High Energy Physics - Dubna

R. Boskovic Institute

Brookhaven National Laboratory

Brookhaven National Laboratory

Mt. Holyoke College

University of Tsukuba

Ruder Boskovic Institute

Inst. for Theoretical and Experimental Physics-Moscow

Lawrence Berkeley National Laboratory

Brookhaven National Laboratory

Institute de Fisica da University de Sao Paulo

Institute of Nuclear Physics-Krakow

Warsaw University, Poland

Indiana University Cyclotron Facility
$954,953,931,914,913,907$

$957,947,919,898$

$931,929,909,907,906,905,890,885,856,813$

850

PHENIX

$954,953,914,913,890$

925

STAR, 895

925,880

STAR

868

STA

788,813

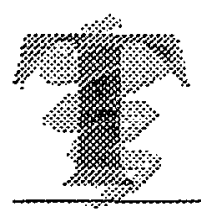

Taeger, S. A.

Tai, A.

Takagui, M.

Takahashi, J.

Takahashi, J.

Takehashi, T.

Takeutchi, F.

Tallerico, $\mathrm{T}$.

Tamagawa, $T$.

Tamagawa, Y.

Tamai, Munetaka

Tamura, $\mathrm{H}$.

Tamura, $\mathrm{K}$.

Tanaka, Kazuhiro

Tanaka, $M$.

Tanaka, Yoshito

Tang, Aihong

Tang, C.

Tang, J.

Tang, J.L.

Tang, L.

Tanida, $\mathrm{K}$.

Tannenbaum, M. J.

Tarchini, A.

Teige, $\mathrm{S}$.

Teng, $L$.

Teng, $R$.

Tepikian, $\mathrm{S}$.

Thein, $\mathbf{D}$.

Themann, $\mathrm{H}$.

Therm, R. E.

Thiessen, A.

University of Notre Dame

University of California at Los Angeles

University of São Paulo

Wayne State University

Institute de Fisica da University de Sao Paulo

Tohoku University

Kyoto-Sangyo University

Brookhaven National Laboratory

University of Tokyo

Fukui University

University of Tokyo

INS-University of Tokyo

Osaka University

KEK

Brookhaven National Laboratory

Nagasaki Inst. of Applied Science

Kent State University

University of New York-Stony Brook

University of Texas

National Central University - Taiwan

Hampton University

University of Tokyo

Brookhaven National Laboratory

Institute Subatomics de Strasbourg

Indiana University

Argonne National Laboratory

University of Rochester

Brookhaven National Laboratory

University of California-LosAngeles

Rice University

Brookhaven National Laboratory

Los Alamos National Laboratory
852

STAR, 941

PHENIX, 877

896

STAR

930

885,813

821

$929,906,905$

949,787

PHENIX

$930,929,917,906,905$

929

PHENIX

850,821

PHENIX

STAR

pp2pp

896

PHOBOS

$930,929,906$

930,906

866

STAR

852

880

PHOBOS

pp2pp, 880

STAR

896

880

931,907 
Thomas, $J$.

Thomas, T. L.

Thompson, A.

Thompson, D. R.

Thompson, J.A.

Thompson, $\mathrm{M}$.

Thompson, P.

Thompson, $\mathrm{R}$.

Throwe, T. G.

Tikhomirov, V.

Timmermans, $\mathrm{C}$.

Tincknell, M. L.

Tippens, W. B.

Tippens, W. B.

Todosow, $\mathbf{M}$.

Togawa, Manabu

Tojo, J.

Tokarev, M.

Toldo, F.

Tominaka, $T$.

Tonjes, $M$.

Tonse, Shaheen

Toropin, A. N.

Torun, $\mathrm{Y}$.

Totzer, $\mathrm{R}$.

Towell, R.

Trainor, T.A.

Trattner, A.

Trbojevic, D.

Trentalage, $S$.

Tribble, $\mathrm{R}$.

Tricoma, A.

Trofimov, A.

Trofimov, V.

Tru, P.

Truoel, P.

Trzaska, $\mathbf{M}$.

Trzupek, A.

Tsai, 0 .

Tsalov, D.

Tschirhart, R.S.

Tserruva, Itzhak

Tsoupas, $N$.

Tsunemi, $T$.

Tsuruoka, Hiroshi

Tsvetkov, I. O.

Tull, C.

Turcot, A. S.

Tveter, T. S.

Tydesjo, Henrik
Lawrence Livermore National Laboratory

University of New Mexico

Dublin Inst. For Advanced Studies

University of Notre Dame

University of Pittsburgh

University of Birmingham

Brookhaven National Laboratory

Bechtal Nevada

Brookhaven National Laboratory

Moscow Engineering Physics Institute

STAR, 910, 866

PHENIX, 950, 926

893

852

865

STAR

821

939,933

864

STAR

821

864

$953,931,907$

Brookhaven National Laboratory

University of California-Los Angeles

Brookhaven National Laboratory

Kyoto University

Kyoto University

Laboratory of High Energy Physics - Dubna

Brookhaven National Laboratory

RIKEN

Michigan State Univesity

Lawrence Livermore National Laboratory

Institute for Nuclear Research

Stony Brook University

University of New Mexico

Los Alamos National Laboratory

Univesity of Washington

Space Sciences Laboratory

Brookhaven National Laboratory

University of California-Los Angeles

Texas A\&M University

University of Catania

Boston University

Moscow Engineering Physics Institute

University Zurich

University of Zurich

SUNY at Stony Brook

Institute of Nuclear Physics-Krakow

University of California-LosAngeles

Pennsylvania State University

Fermi National Accelerator Laboratory

Weizmann Inst. of Science

Brookhaven National Laboratory

High Energy Accel. Research Organization (KEK)

University of Tsukuba

$954,927,924,914,913,909,890$

939

PHENIX 950

STAR

821

880

STAR

PHENLX

940

951,910

813

PHENIX

STAR

STAR

932

STAR, 925, 896

STAR

896

821

STAR

926

865

877

PHOBOS, 868

STAR

850

949

PHENIX

$925,880,820$

949

PHENIX

850

STAR

787

TRIUMF

BRAHMS

University of Oslo-Norway

PHENIX 
Udumaki, Takeyoshi

Ufimtsev, A.

Ukai, $\mathrm{K}$.

Ullmann, J.

Underwood, D.

Umer, D.

Ushiroda, Tsukasa
Hiroshima University

Institute for High Energy Physics-Serpukhov

INS-University of Tokyo

Los Alamos National Laboratory

Argonne National Laboratory

University of Illinois

Nagasaki Inst. of Applied Science
PHENIX

pp2pp, 880

787

939

STAR, 950, 925, 880

924,821

PHENIX

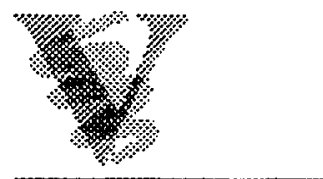

Vale, C.M.

Massachusetts Inst. Technology

PHOBOS

van Asselt, W.

Brookhaven National Laboratory

van Nieuwenhuizen, G. University of Illinois - Chicago

PHOBOS

VanBibber, K. A.

Lawrence Livermore National Laboratory

933

Van Buren, G.

University of California at Los Angeles

941,864

VanGinneken, $\mathrm{A}$.

Fermi National Laboratory

951

VanderMolen, A.

Michigan State Univesity

STAR

Vanderwerp, $J$

van Oers, W.

Indiana University

948

Vardanyan, I. N.

University of Manitoba

Moscow State University

$906,885,813$

University of Texas-Arlington

852

Vartapetian, A.

Vasilevski, I.M.

Particle Physics Laboratory - Dubna

$\mathrm{pp} 2 \mathrm{pp}$

Institute for High Energy Physics-Protvino

STAR

Vasiliev, A. N.

Texas A\&M University

TAR, 925

STAR

Vazquez, M.

Verdier, $R$.

Brookhaven National Laboratory

Massachusetts Inst. Technology

957, 947, 919, 898

Inst. for High Energy Physics

PHOBOS

Victorov, V.

Vidal, $M$.

Max-Planck-Institute fuer Physik

949

Videbaek, F.

Brookhaven National Laboratory

STARo

Vigdor, S. E.

Indiana University

Vinogradov, Aleksandr Kurchatov Institute, Russia

Viola, V. E.

Indiana University

Brookhaven National Laboratory

Viren, $B$.

Space Science Laboratory

Virgnia Polytechnic Institute

Kurchatov Institute, Russia

University of Pittsburgh

Wayne State University

University of Heidelberg

SUNY at Stony Brook

Lawrence Berkeley Laboratory

BRAHMS, 866

STAR, 948

PHENLX

900

949

896

926

Volkov, Maxim

Voloshin, S.

Voloshin, Sergei

von Walter, P.

Vongpaseuth, $\mathrm{T}$.

$\mathrm{Vu}, \mathrm{C}$.

Petersburg Nuclear Physics Institute, Russia

PHENIX

877

STAR

821

877

STAR

PHENLX 
Wada, $\mathbf{R}$.

Waddington, C. J.

Wadsworth, B.

Wakasa, $\mathrm{T}$.

Waldren C.

Wales, W. D.

Walsh, $\mathrm{M}$.

Wang, Enke

Wang, $F$.

Wang, $F$.

Wang, $\mathrm{H}$.

Wang, $H$.

Wang, Hui

Wang, Qin

Wang, Qin

Wang, $\mathrm{S}$.

Wang, Xiaorong

Wang, $Y$.

Wang, $Y$.

Warburton, D.

Watanabe, N.

Ward, $\mathrm{H}$.

Ward, T.

Ward, $T$.

Ware, C. B.

Warner, $T$.

Watanabe, N.

Waters, $\mathrm{L}$.

Watson, J. W.

Wefel, J. P.

Weggel, R.

Weinstein, $R$.

Weise, $R$.

Wells, R.

Welsh, $\mathrm{R}$.

Welsh, R.

Wcssels, J.

Westfall, G.

Westphal, A.

Weyer, $\mathrm{H}$.

Weygand, D. P.

Wieder, $\mathrm{M}$.

Wieman, $\mathrm{H}$.

White, D.

White, D. B.

Whitfield, J.

Whitehouse, D.

Whitten, C.

Whohn, F.

Widenbeck, M. E.

Wider, $\mathrm{M}$.
Texas A\&M University

University of Minnesota

Massachusetts Inst. Technology

University of Tokyo

Colorado State University

University of Pennsylvania

Creighton University

Inst. Particle Physics - China

Columbia University

Purdue University

Brookhaven National Laboratory

University of California-Riverside

Georgia State University

Inst. Particle Physics - China

Georgia State University

Kent State University

Georgia State University

Columbia University

University of Texas - Austin

Brookhaven National Laboratory

Japan Atomic Energy Reserach Institute

University of Texas-Austin

Brookhaven National Laboratory

U. S. Department of Energy

University of Texas-Austin

Boston University

Japan Atomic Energy Research Institute

Los Alamos National Laboratory

Kent State University

Louisiana State University

Brookhaven National Laboratory

University of Houston

Tel-Aviv University

Ohio State University

University of Michigan

College of William and Mary

SUNY at Stony Brook

Michigan State Univesity

University of California-Berkeley

Paul Scherrer Institute

Brookhaven National Laboratory

Freiburg University

Lawrence Berkeley Laboratory

Rensselaer Polytechnic Institute

University of California-Los Angeles

Carnegie-Mellon University

Boston University

University of California-Los Angeles

Iowa State University

Caltech

Freiburg University
BRAHMS

$934,869,868$

PHOBOS

925

$957,919,898$

940

STAR

STAR

866

STAR

951

917

PHENIX

STAR

PHENIX

895

PHENIX

866

STAR

821

938

STAR, 931, 907

820

939

871

811

838

939

STAR, 948

868

951

904

835

STAR, 895

896

$935,924,871$

877

STAR

915

865

852

813

STAR

852

$914,913,909,890$

895

811

STAR, 925

941

934

811 
Wienands, $U$.

Wienold, $T$.

Wilczynska, B.

Wilczynski, $\mathrm{H}$.

Wilkins, $\mathbf{R}$.

Williams, $N$.

Willson, $R$.

Willutzki, H. J.

Wilson, $\mathrm{J}$.

Wilson, $\mathrm{K}$.

Winn, D.

Winter, D.

Wise, J.

Wissink, S. W.

Witkowski, M.

Witt, $\mathbf{R}$.

Witzig, C.

Wohn, F. K.

Wojcicki, S. G.

Wolfe, D.

Wolfe, M.

Wolfs, F.

Wolin, E.

Wolter, W.

Woodruff, D.

Woody, C.

Worm, S.

Worstell, W.

Wosiek, B.

Wozniak, K.

Wright, D. M.

$\mathrm{Wu}, \mathrm{J}$.

$\mathrm{Wu}, \mathrm{J}$. Y.

Wu, Y.

Wu, Yuanfang

Wuosmaa, A.

Wyslouch, B.
TRIUMF

880

Lawrence Berkeley National Laboratory

Institute of Nuclear Physics-Krakow

Institute of Nuclear Physics-Krakow

Prairie View A\&M University

Brookhaven National Laboratory

Ohio State University

Brookhaven National Laboratory

University of Houston

Wayne State University

Fairfield University

Columbia University

Northwestern University

Indiana University

Rensselaer Polytechnic Institute

Kent State University

Brookhaven National Laboratory

Iowa State University

Stanford University

University of New Mexico

University of New Mexico

University of Rochester

College of William and Mary

Institute of Nuclear Physics-Krakow

Massachusetts Institute of Technology

Brookhaven National Laboratory

University of Texas-Austin

Boston University

Institute of Nuclear Physics-Krakow

Institute of Nuclear Physics-Krakow

Lawrence Livermore National Laboratory

University of Washington

Pennsylvania State University

Columbia University

Inst. Particle Physics - China

Argonne National Laboratory

Massachusetts Inst. Technology
895

868

868

957

880

STAR

852

940

896

821

910

852

STAR, 948

935,852

STAR, 895

949,787

864

871

950

$906,865,813,811$

PHOBOS, 917

871

868

PHOBOS, 866

926,871

871

821

PHOBOS, 868

PHOBOS, 868

933

STAR

850

866

STAR

PHOBOS; 917

PHOBOS

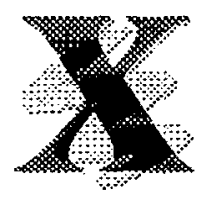

Xiang, $\mathrm{H}$.

Xie, Wei

Xiong, $\mathrm{H}$.

$\mathrm{Xu}, \mathrm{G}$.

$\mathrm{Xu}, \mathrm{N}$.

$\mathrm{Xu}, \mathrm{Q}$.

$\mathrm{Xu}, \mathrm{Z}$.
University of California-Riverside

University of California - Riverside

Wayne State University

PHENTX

University of California-Riverside

Lawrence Berkeley Laboratory

University of California-Riverside

Yale University
877

917

STAR

866

941 
Yagi, K.

Yakutin, A.

Yamamoto, A.

Yamamoto, E.

Yamamoto, E.

Yamamoto, K.

Yang, $\mathrm{X}$.

Yang, T. C-H.

Yang, $\mathrm{Yi}$

Yanovich, Andrey

Yao, $\mathrm{H}$.

Yasuno, $\mathrm{T}$.

Yates, G. J.

Yavin, A. I.

Yennello, S. J.

Yepes, P.

Yershov, A. A.

Yershov, N.V.

Yoder N. R.

Yokkaichi, S.

Yokkaichik Satoshi

Yokosawa, A.

Yokoya, K.

Yoshi, M.

Yoshimura, Y.

Yoshioka, T.

Yosoi, $\mathrm{M}$.

Youn, M.

Yu, Mciling

Yuan, L.

Yurevich, V.

Yushmanov, Igor
University of Tsukuba

Institute High Energy Physics-Protvino

National Laboratory for High Energy Physics (KEK)

Lawrence Berkeley Laboratory

University of California at Los Angeles

Kyoto University

Columbia University

NASA Johnson Space Center

China Inst. of Atomic Energy

Inst. for High Energy Pysics, Russia

Massachusetts Institute of Technology

High Energy Accel. Research Organ. (KEK)

Los Alamos National Laboratory

Tel-Aviv University

Texas A\&M University

Rice University

Moscow State University

Inst. for Nuclear Research

Indiana University

University of Kyoto

RIKEN

Argonne National Laboratory

KEK

KEK, National Laboratory for High Energy Physics

KEK, National Laboratory for High Energy Physics

High Energy Accel. Research Organization (KEK)

Kyoto University

University of Houston

Inst. Particle Physics - China

Hampton University

Laboratory of High Energy Physics - Dubna

Kurchatov Institute, Russia
866

STAR

821

STAR

941

$950,906,885$

910,866

919,898

PHENLX

PHENIX

917,866

949

933

835

900

STAR, 896

852

949

900

813

PHENIX

STAR, 925,880

880

932

949,787

949

906,885

940, 929

STAR

906

STAR

PHENIX
Zaharia, R.

Zajc, W. A.

Zalewski, K.

Zberecki, K.

Zborovsky, I.

Zeller, M.

Zenevsky, Y.

Zeps, V.

Zeps, V.

Zgura, I.S.

Zhang, $B$.
University of Bucharest-Romania

Columbia University

Jagellonian University, Poland

Warsaw University, Poland

Laboratory of High Energy Physics - Dubna

Yale University

Laboratory of High Energy Physics - Dubna

University of Kentucky

Carnegie-Mellon University

University of Bucharest-Romania

Institute High Energy Physics - Beijing
BRAHMS

910,866

PHOBOS

STAR

STAR

926, 865

STAR

$931,907,885$

813

BRAHMS

STAR 


$\begin{array}{ll}\text { Zhang, Chun } & \text { Columbia University } \\ \text { Zhang, Haibin } & \text { Yale University } \\ \text { Zhang, W. M. } & \text { Kent State University } \\ \text { Zhang, W. N. } & \text { Harbin University } \\ \text { Zhang, Y. } & \text { SUNY at Stony Brook } \\ \text { Zhang, Y. } & \text { Columbia University } \\ \text { Zhang, Zhou } & \text { State University of New York at Stony Brook } \\ \text { Zhao, D. } & \text { Northwestern University } \\ \text { Zhao, K. } & \text { City College of New York } \\ \text { Zhao, Y. } & \text { Brookhaven National Laboratory } \\ \text { Zhidkov, N. . } & \text { Particle Physics Laboratory - Dubna } \\ \text { Zhou, Suijian } & \text { Weizmann Inst. of Science } \\ \text { Zhu, F. } & \text { Brookhaven National Laboratory } \\ \text { Zhu, Q. } & \text { University of California-Riverside } \\ \text { Zhu, Y. } & \text { City College of New York } \\ \text { Zieminska, D. } & \text { Indiana University } \\ \text { Ziliak, Z. } & \text { Indiana University } \\ \text { Zimmerman, D. } & \text { University of Minnesota } \\ \text { Ziock, H. } & \text { Los Alamos National Laboratory } \\ \text { Zou, C. } & \text { SUNY at Stony Brook } \\ \text { Zou, C. } & \text { University of California-Riverside } \\ \text { Zoulkarneev, R. } & \text { Particle Physics Laboratory - Dubna } \\ \text { Zubarev, A. } & \text { Laboratory of High Energy Physics - Dubna } \\ \text { Zumbro, J. } & \text { Los Alamos National Laboratory } \\ \text { zu Putlitz, G. } & \text { University of Heidelberg } \\ \text { Zybert, R. } & \text { University of Birmingham } \\ \text { Zychowski, P. } & \text { Institute Nuclear Physics, Poland } \\ & \\ & \end{array}$


